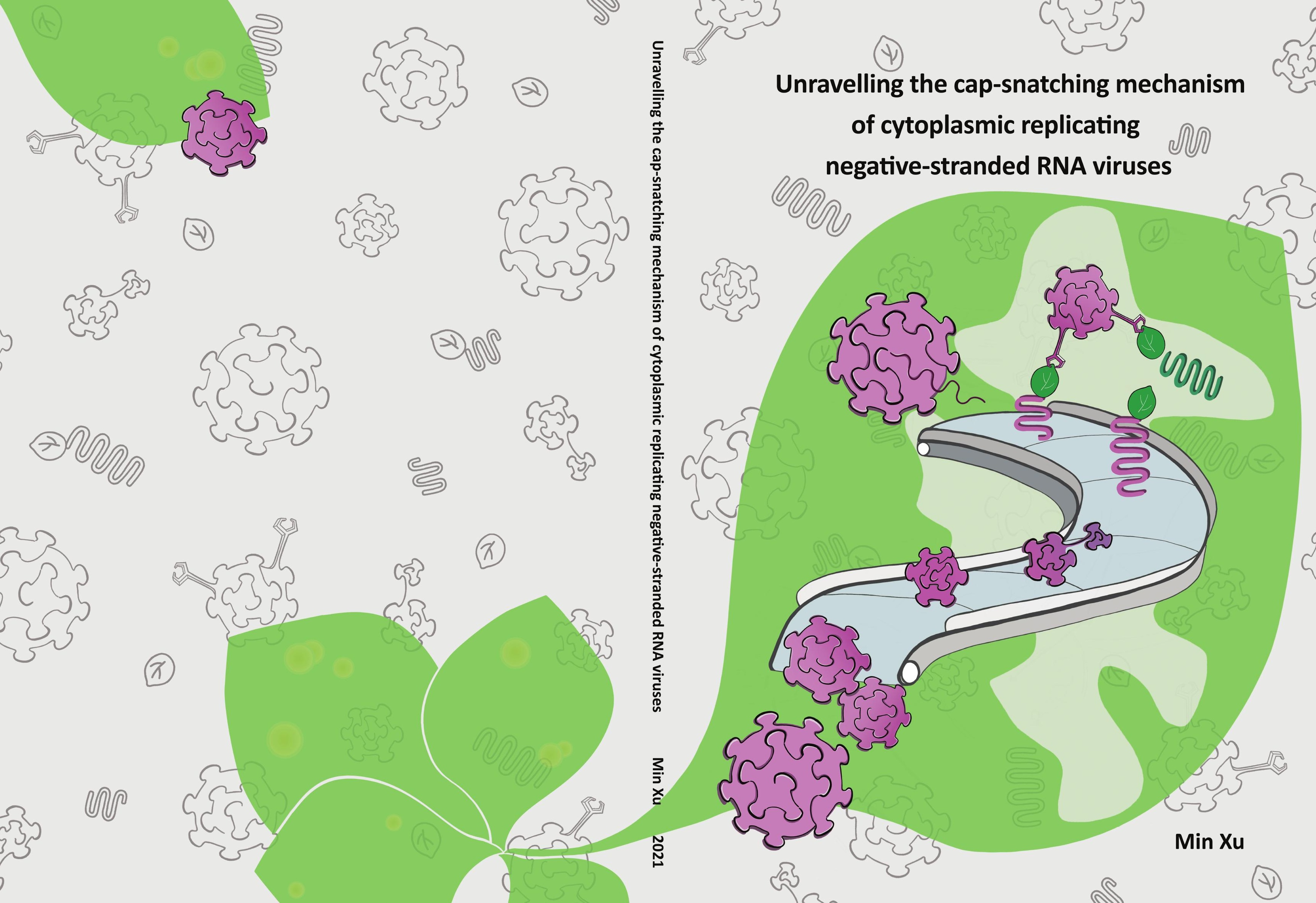




\section{Propositions}

1. Cap-snatching causes a host translational 'shut-off'.

(this thesis)

2. The hijacking of RNA sources for cap-snatching is conserved/generic for all plant- and animal-infecting cytoplasmic-replicating negative-strand, segmented RNA viruses.

(this thesis)

3. Model species are not representative.

4. Pilot experiments are more rewarding than repetitions.

5. Wealth redistribution is the basis of social stability.

6. The COVID-19 associated lockdowns did people realize how important inperson communication is.

Propositions belonging to the thesis, entitled

"Unravelling the cap-snatching mechanism of cytoplasmic replicating negative-stranded RNA viruses"

Min Xu, Wageningen, 6 October 2021 


\section{Unravelling the cap-snatching mechanism of cytoplasmic replicating negative- stranded RNA viruses}

Min Xu 


\section{Thesis committee}

\section{Promotors}

Prof. Dr M.M. van Oers

Professor of Virology

Wageningen University \& Research

Dr R.J.M. Kormelink

Associate professor, Laboratory of Virology

Wageningen University \& Research

Prof. Dr X. Tao

Professor of Molecular Plant Virology

Nanjing Agricultural University, China

\section{Other members}

Prof. Dr J.E. Kammenga, Wageningen University \& Research

Prof. Dr C.M.J. Pieterse, Utrecht University

Dr B. Krenz, Leibniz-Institut, Deutsche Sammlung von Mikroorganismen und Zellkulturen $\mathrm{GmbH}$, Germany

Dr M. Kikkert, Leiden University Medical Center

This research was conducted under the auspices of the Graduate School Experimental Plant Science 


\title{
Unravelling the cap-snatching mechanism of cytoplasmic replicating negative- stranded RNA viruses
}

\author{
Min Xu
}

Thesis

submitted in fulfilment of the requirements for the degree of doctor at Wageningen University by the authority of the Rector Magnificus,

Prof. Dr A.P.J. Mol, in the presence of the

Thesis Committee appointed by the Academic Board to be defended in public on Wednesday 6 October 2021 at 11 a.m. in the Aula. 
Min Xu

Unravelling the cap-snatching mechanism of cytoplasmic replicating negative-stranded RNA viruses

172 pages.

PhD thesis, Wageningen University, Wageningen, the Netherlands (2021)

With references, with summary in English

ISBN: 978-94-6395-912-4

DOI : https://doi.org/10.18174/550609 


\section{Table of content}

$\begin{array}{lll}\text { Chapter } 1 & \text { General introduction } & 7\end{array}$

Chapter 2 Cellular RNA hubs: friends and foes of plant viruses 19

Chapter $3 \quad$ Dynamic transcriptional profiles of Arabidopsis $\quad 45$ thaliana infected by Tomato spotted wilt virus

Chapter $4 \quad$ Cytoplasmic sources of capped RNA for genome $\quad 69$ transcription initiation of cytoplasmic replicating, segmented negative strand RNA viruses

Chapter 5 High throughput sequencing of snatched host cellular mRNA leader sequences for viral genome transcription initiation and identification of the host donor genes

Chapter 6 General discussion

Appendix Reference list

Summary

Acknowledgements

About the author

Publication list 

Chapter 1

\section{General introduction}

Min Xu
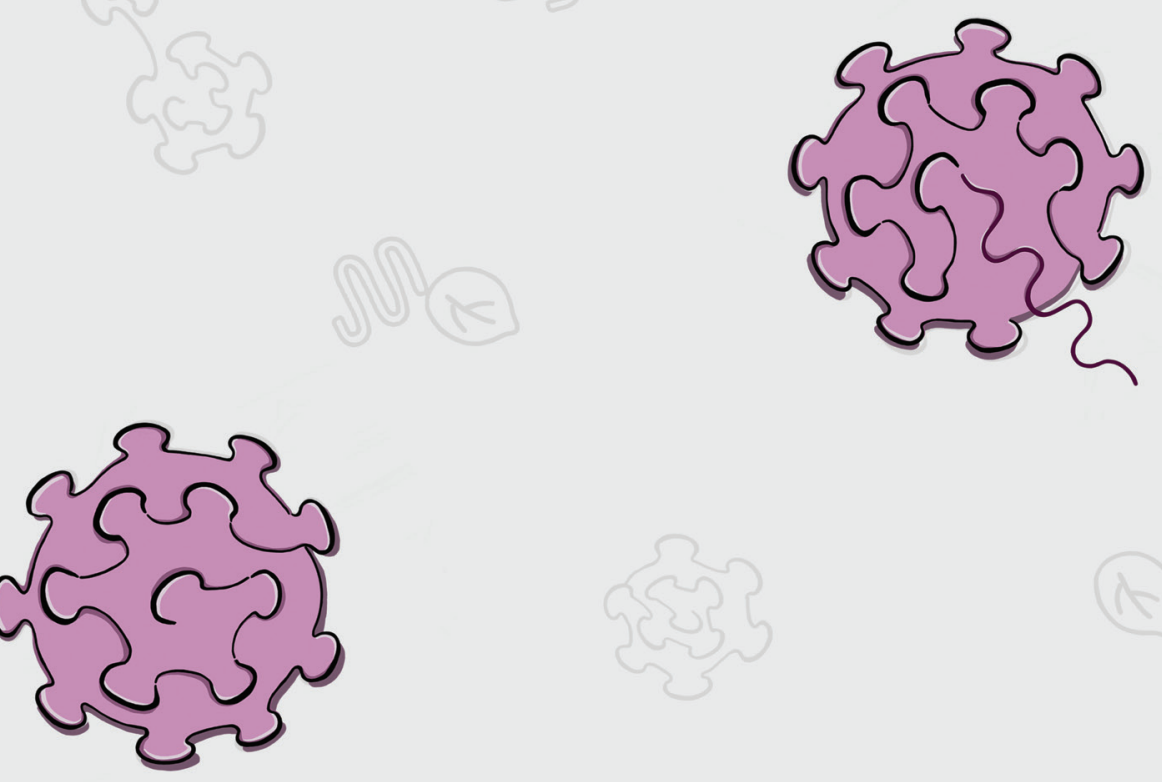


\section{Introduction}

Negative strand RNA viruses (NSVs) generally split up in two major groups. Those containing a non-segmented RNA genome are classified in the order of Mononegavirales and include families like the Paramyxoviridae, Rhabdoviridae and Filoviridae. Among their members are medically important and biologically threatening viruses like measles, rabies and ebola. At the start of this thesis research most NSVs with a segmented RNA genome classified into the families Orthomyxoviridae, Arenaviridae, and Bunyaviridae, and into several floating genera that were not assigned to any family yet, like the tenuiviruses, emaraviruses, and ophioviruses. Recently, most of the latter families and genera, with the exception of the family Orthomyxoviridae and the genus Ophiovirus (now family Ophioviridae), have been reclassified into the order Bunyavirales (Table 1.1) (Abudurexiti et al. 2019). Viruses with a high impact on public health and the economy, are also found among these segmented NSVs, such as influenza virus (family Orthomyxoviridae). Influenza viruses are a common cause of (seasonal) human respiratory infections and responsible for considerable morbidity and mortality in humans, thereby forming an important burden on national healthcare budgets (Taubenberger and Morens 2008). Segmented NSVs, besides animal and human-infecting members, also include viruses that cause diseases in plants and may lead to large yield losses in crops and character defects in ornamentals. Amongst those are the tospoviruses (family Tospoviridae) and tenuiviruses (family Phenuiviridae). While tenuiviruses, with Rice stripe virus (RSV) as well-known representative, cause major diseases in rice cultivations, tospoviruses with Tomato spotted wilt virus (TSWV) as type species, infect more than 1000 plant species within more than 80 families worldwide (Scholthof et al. 2011). Although being widely distributed in (sub)tropical climate regions, tospoviruses also occur in the more temperate regions, but there tospovirus infections are primarily restricted to greenhouse cultivations where, besides horticultural crops, they also affect ornamentals like chrysanthemum, alstroemeria, iris and impatiens.

\section{Genome organization and expression strategy of segmented NSVs}

Besides differences in host range and serological relationships, one of the major differences between the various groups of segmented NSVs is their number of genomic RNAs. Members of the Bunyavirales contain a genome consisting of 2-8 genomic segments while members of the Ophioviridae contain 3-4 genomic segments, and those of the Orthomyxoviridae 6-8 genomic segments (Table 1.1). Furthermore, several members of the Bunyavirales contain genetic elements of ambisense polarity that carry two genes on opposite strands 
that are expressed via the synthesis of subgenomic mRNAs. On the other hand, the genetic elements of the Ophioviridae and Orthomyxoviridae are all of entire negative polarity. The total genome of all these viruses is over $10 \mathrm{~kb}$ in size, with a coding capacity for several (414) viral proteins. For all segmented NSVs the largest genome segment encodes the viral RNA-dependent RNA polymerase (RdRp). However, the fully functional RNA polymerase of Orthomyxoviridae is a complex composed of three subunits, namely, PA, PB1 and PB2 (Stevaert and Naesens 2016), and these subunits are also encoded by the three largest genomic RNA segments.

Table 1.1. Characteristics of families and a single floating genus in segmented NSVs. Approved by ICTV, 2018 (Abudurexiti et al. 2019).

\begin{tabular}{|c|c|c|c|c|c|c|}
\hline Order & Family/genus & Representative(s) & $\begin{array}{l}\text { Genome } \\
\text { Segments }\end{array}$ & $\begin{array}{l}\text { Genome } \\
\text { Size }\end{array}$ & $\begin{array}{l}\text { Virion } \\
\text { morphology }\end{array}$ & Host/vector \\
\hline \multirow{12}{*}{ Bunyavirales } & Narioviridae & $\begin{array}{c}\text { Crimean-Congo } \\
\text { haemorrhagic fever } \\
\text { orthonairovirus }\end{array}$ & 3 & $\sim 18 \mathrm{~kb}$ & $\begin{array}{l}\text { Enveloped, } \\
\text { spherical }\end{array}$ & $\begin{array}{c}\text { Human/ } \\
\text { invertebrate }\end{array}$ \\
\hline & Peribunyaviridae & $\begin{array}{l}\text { Schmallenberg } \\
\text { orthobunyavirus }\end{array}$ & 3 & $11-19 \mathrm{~kb}$ & $\begin{array}{c}\text { Enveloped, } \\
\text { spherical }\end{array}$ & $\begin{array}{c}\text { Human/ } \\
\text { invertebrate }\end{array}$ \\
\hline & Hantaviridae & $\begin{array}{c}\text { Sin Nombre } \\
\text { orthohantavirus }\end{array}$ & 3 & $11-20 \mathrm{~kb}$ & $\begin{array}{c}\text { Enveloped, } \\
\text { spherical }\end{array}$ & Human \\
\hline & Leishbuviridae & $\begin{array}{l}\text { Leptomonas } \\
\text { shilevirus }\end{array}$ & 3 & $11-20 \mathrm{~kb}$ & $\begin{array}{c}\text { Enveloped, } \\
\text { spherical }\end{array}$ & Human \\
\hline & Phasmaviridae & $\begin{array}{l}\text { Kigluaik phantom } \\
\text { orthophasmavirus }\end{array}$ & 3 & $12-13 \mathrm{~kb}$ & $\begin{array}{c}\text { Enveloped, } \\
\text { spherical }\end{array}$ & Invertebrate \\
\hline & Phenuiviridae & $\begin{array}{c}\text { Rift Valley fever } \\
\text { phlebovirus, Rice } \\
\text { stripe virus }\end{array}$ & $3-8$ & $11-25 \mathrm{~kb}$ & $\begin{array}{l}\text { Enveloped, } \\
\text { spherical }\end{array}$ & $\begin{array}{c}\text { Human/ } \\
\text { plant/ } \\
\text { invertebrate }\end{array}$ \\
\hline & Arenaviridae & $\begin{array}{c}\text { Lassa } \\
\text { mammarenavirus }\end{array}$ & 2 & $\sim 11 \mathrm{~kb}$ & $\begin{array}{c}\text { Enveloped, } \\
\text { spherical }\end{array}$ & Human \\
\hline & Tospoviridae & $\begin{array}{c}\text { Tomato spotted wilt } \\
\text { virus }\end{array}$ & 3 & $\sim 17 \mathrm{~kb}$ & $\begin{array}{c}\text { Enveloped, } \\
\text { spherical }\end{array}$ & $\begin{array}{c}\text { Plant/ } \\
\text { invertebrate }\end{array}$ \\
\hline & Fimoviridae & $\begin{array}{c}\text { European mountain } \\
\text { ash ringspot- } \\
\text { associated virus }\end{array}$ & $5-8$ & $12-13 \mathrm{~kb}$ & $\begin{array}{l}\text { Enveloped, } \\
\text { spherical }\end{array}$ & Plant/mites \\
\hline & Cruliviridae & Crustacean lincruvirus & 3 & $\sim 15 \mathrm{~kb}$ & $\begin{array}{l}\text { Enveloped, } \\
\text { spherical }\end{array}$ & Crustacean \\
\hline & Mypoviridae & Myriapod hubavirus & 3 & $\sim 16 \mathrm{~kb}$ & $\begin{array}{c}\text { Enveloped, } \\
\text { spherical }\end{array}$ & Invertebrate \\
\hline & Wupedeviridae & Millipede wumivirus & 3 & $\sim 20 \mathrm{~kb}$ & $\begin{array}{c}\text { Enveloped, } \\
\text { spherical }\end{array}$ & Arthropod \\
\hline Articulavirales & Orthomyxoviridae & Influenza A virus & $6-8$ & $\begin{array}{c}10- \\
14.6 \mathrm{~kb}\end{array}$ & $\begin{array}{c}\text { Enveloped, } \\
\text { spherical }\end{array}$ & $\begin{array}{c}\text { Human/ } \\
\text { invertebrate }\end{array}$ \\
\hline Unassigned & Ophioviridae & $\begin{array}{l}\text { Citrus psorosis } \\
\text { ophiovirus }\end{array}$ & $3-4$ & $\begin{array}{c}11.3-12.5 \\
\mathrm{~kb}\end{array}$ & $\begin{array}{l}\text { Unenveloped, } \\
\text { highly } \\
\text { flexuous }\end{array}$ & Plant \\
\hline
\end{tabular}


Despite differences in the genomic organization and protein composition, segmented NSVs also share a number of characteristics. Firstly, the virion of most viruses is more or less spherical in shape with a diameter in the range of $80-200 \mathrm{~nm}$ and surrounded by a lipid envelope (with the exception of tenuiviruses and ophioviruses). The envelop contains two major viral glycoproteins that form spikes and play a major role in virus entry. A particular characteristic of all NSVs is a ribonucleoprotein complex (RNP), consisting of the viral RNA segments tightly enwrapped by a nucleocapsid protein (also called nucleoprotein) and a few copies of the viral RdRp (Fig 1.1A) (Hastie et al. 2011; Kormelink et al. 2011; Niu et al. 2013; Ortin and Martin-Benito 2015). While the genomic RNAs of NSVs, being of negative or ambisense polarity, are not infectious, their RNPs are the minimal infectious units to initiate viral replication and transcription.

The replication and transcription strategy of a model segmented NSV with a tripartite negative/ambisense RNA genome, representative for a large number of viruses from the Bunyavirales, is shown in Fig. 1.1B. Replication of the viral genomic RNAs (vRNAs) occurs by the replicase activity of the RdRp. Expression of the open reading frames (ORFs) occurs via the synthesis of near genomic length mRNAs (from RNA segments with negative polarity) or subgenomic length mRNAs (in case of ambisense RNA segments).

Unique to almost all segmented NSVs is that their RdRp lacks a methyltransferase capacity that is needed to provide a $5^{\prime}$-cap-structure to the $5^{\prime}$ end of viral mRNAs and render them translatable. The $5^{\prime}$-cap-structure is also referred as a 7-methylguanylate cap, which is a methylated guanine nucleotide (Shatkin 1976). To circumvent the lack of a methyltransferase, these viruses employ a capping mechanism called cap-snatching (Decroly et al. 2011). Despite differences in spatial distribution of the replication process (nucleus versus cytoplasm), or the host range (animal vs plant), cap-snatching among segmented NSVs shows several features that appear highly conserved and are applicable to all known animal- and plant-infecting segmented NSVs.

During this process, the viral RdRp cleaves short capped-RNA leader sequences from host cellular mRNAs and uses those as primers to initiate viral mRNA synthesis (Fig. 1.1C). To enable the cleavage process, the RdRps of all segmented NSVs contain an N-terminal domain with endonuclease activity. Meanwhile, the core of the RdRp contains the conserved catalytic motifs for polymerase activity to achieve RNA synthesis. Whereas a cap-binding domain is present in one of the Influenza virus polymerase subunits as further explained in the next section, no such domain seems to be present in the RdRps of all other segmented 
A

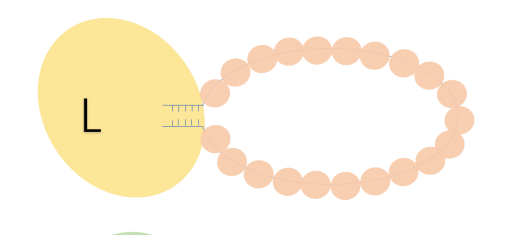

PA

PB2

PB1
B

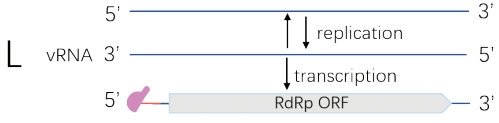

M
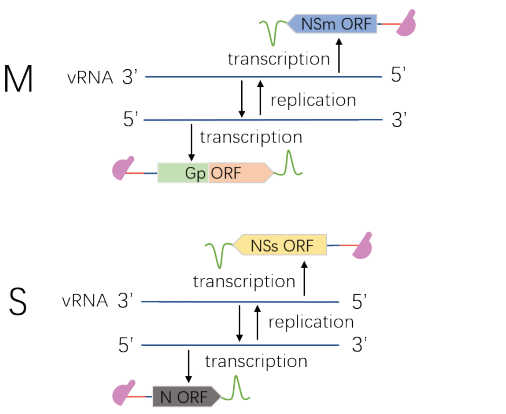

C

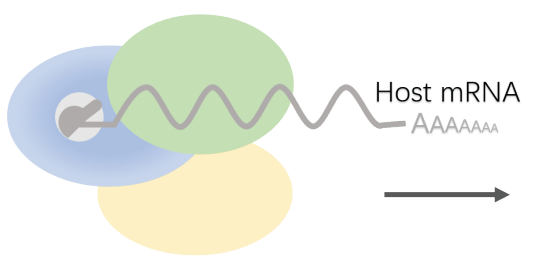

Cap binding

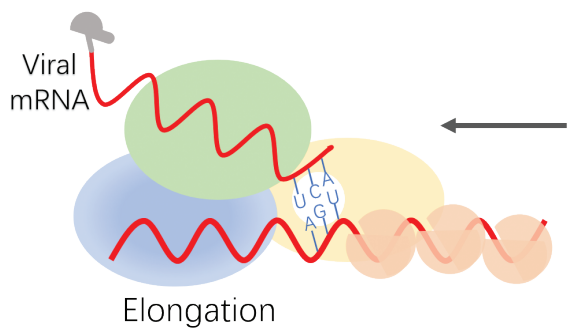

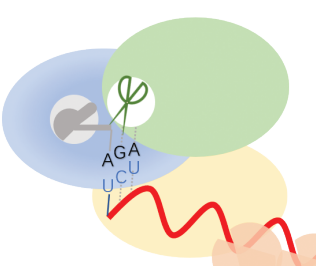

Cap cleavage<smiles>CCCCCCCNON=O</smiles>

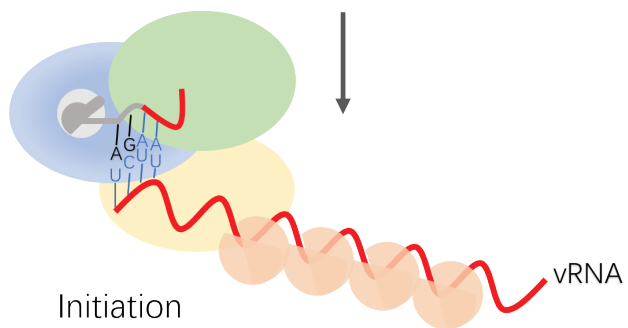

Figure 1.1 RNPs composition and transcription strategy. (A) structure of RNPs, from bunyavirus and influenza virus, respectively; (B) replication and transcription strategy of a bunyavirus; (C) The four major steps during genome transcription initiation of segmented NSVs.

NSVs from known reports (Reguera et al. 2010). During studies of the cap-snatching mechanism, conserved characteristics and minor variations have been found among different segmented NSVs. Snatched capped-RNA leader sequences are relatively short but in general appear to differ in length between segmented NSVs. For influenza viruses, hantaviruses, peribunyaviruses, tospoviruses and tenuiviruses, pairing of single or several bases between the $3^{\prime}$ end residues of the capped-RNA leader and the terminal base(s) of 
the viral RNA template has been demonstrated to promote the use of cap-donors (Duijsings et al. 2001; Garcin et al. 1995; Geerts-Dimitriadou et al. 2011a; Sikora et al. 2017; Yao et al. 2012). Since the process of cap-snatching is highly conserved in all segmented NSVs, it presents an attractive target for future antiviral drug design.

\section{Cap-snatching by the nuclear-replicating Influenza A virus}

Cap-snatching has first been discovered for Influenza virus and it still remains one of the best studied viruses in this respect. In contrast to most segmented NSVs, Influenza is the only virus that replicates in the nucleus (Fig. 1.2). In the nucleus, host capped-RNA leaders 10-13 nucleotides ( $\mathrm{nt}$ ) in size are snatched from nascent mRNAs (Geerts-Dimitriadou et al. 2011a; Li et al. 2001), during which the ability to base-pair to the terminal 3'-UCGU residues of the viral RNA template, promotes the use of specific leader sequences (GeertsDimitriadou et al. 2011b). Furthermore, leader sequences of host mRNAs, in which a basepairing A residue is positioned 10 or $11 \mathrm{nt}$ downstream of the $5^{\prime}$ cap, are preferred (GeertsDimitriadou et al. 2011b). Leader sequences containing a dinucleotide $A G$ at those positions are even more favorable, and are able to outcompete those only containing a single (A) base-paring residue, even when offered in ten-fold lower amounts (Geerts-Dimitriadou et al. 2011b). Leaders containing a G-residue positioned 10 or 11 nt downstream of the 5' cap are also used as donor, and internally prime on the penultimate $C$ residue of the viral RNA template sequence (Geerts-Dimitriadou et al. 2011a). Interestingly, in some cases snatched capped-RNA leaders undergo a prime-and-realign mechanism (PAR), a process during which these capped-RNA leaders first become elongated by one or two nucleotides before they realign backwards and become fully elongated. PAR occurs frequently during cap-snatching, and often is observed with relatively short capped-RNA leaders. The occurrence of PAR explains the presence of repetitive sequences observed in the non-viral leader sequences of viral mRNAs from many different viruses (Duijsings et al. 2001; Garcin et al. 1995; GeertsDimitriadou et al. 2011a; Sikora et al. 2017; Yao et al. 2012).

Most steps during cap-snatching have been well described for Influenza virus and are being performed by the polymerase complex. The three viral subunits of Influenza polymerase, PA, PB1 and PB2, have been assigned specific roles in this process. During cap-snatching PB2 binds to the $5^{\prime}$ cap of a host pre-mRNA, while the endonuclease activity containing PA, next cleaves the host pre-mRNA around 9-13 nt downstream of the 5' cap to release a cappedRNA leader sequence (Fig. 1.1C) (Guilligay et al. 2008; Stevaert and Naesens 2016). After 
alignment of the capped-RNA leader sequence on the viral RNA template, PB1 containing the catalytic polymerase core domain (Pflug et al. 2014), elongates the primer sequence and synthesizes the viral mRNAs (Fig. 1.1C). These viral mRNAs distinguish from (anti)genomic RNA molecules by the presence of a non-viral leader sequence with a 5' cap. Cap-snatching of influenza virus occurs in the nucleus. To this end, the PA unit binds to the carboxyterminal domain (CTD) of host RNA polymerase II (pol II) (Fig. 1.2). Through interaction with the serine-5-phorphorylated form of pol II, influenza virus gains easy access to a pool of $5^{\prime}$ cap structures of nascent messenger transcripts (Engelhardt et al. 2005). Amongst these, noncoding RNAs (ncRNAs) and small nuclear RNAs (sncRNAs) present a preferred source of capped RNA leaders (Gu et al. 2015; Koppstein et al. 2015).

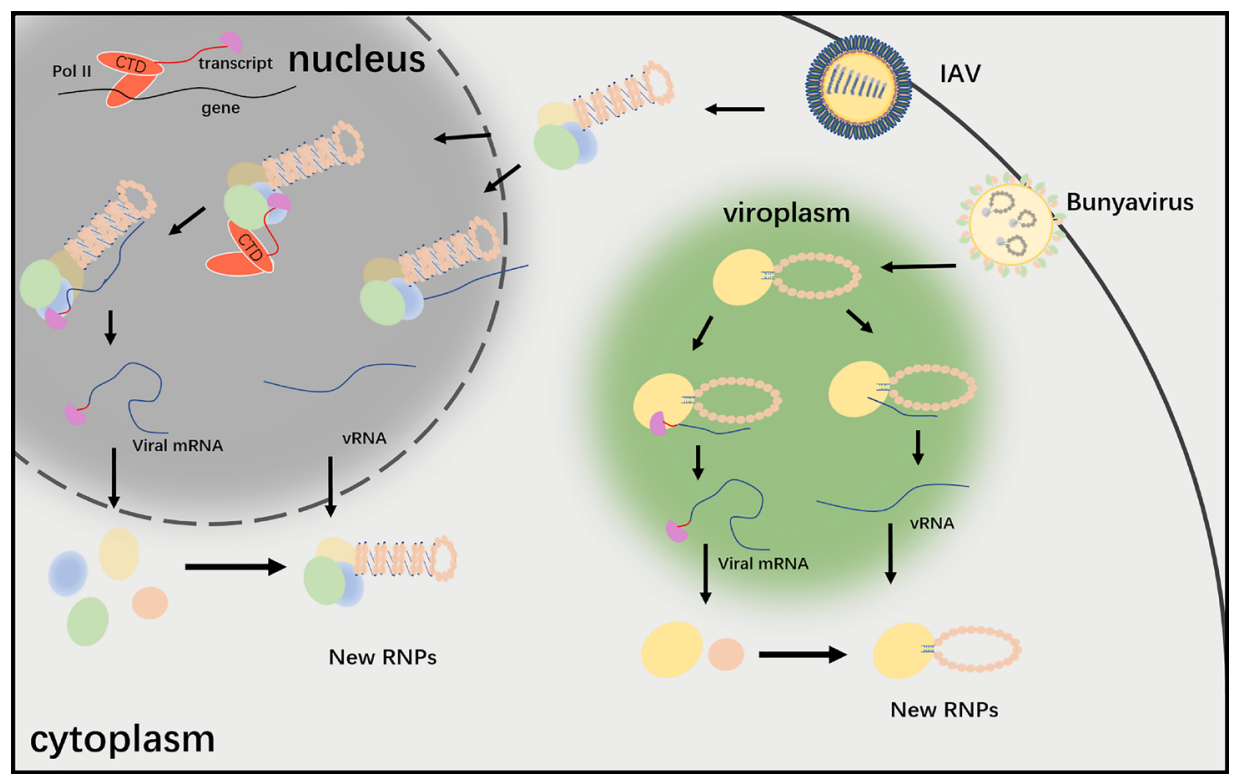

Figure 1.2 Transcription process of segmented NSVs in host cells. Cap-snatching for influenza virus occurs in the nucleus (shown at the left hand side), while for Bunyaviruses occurs in the cytoplasm (right hand side).

\section{Cap-snatching by the cytoplasmic-replicating segmented NSVs}

Also the cytoplasmic replicating, segmented NSVs exhibit a preference for capped-RNA leader molecules that are able to align on the 3' viral genomic RNA by virtue of base-pairing (Duijsings et al. 1999, 2001; Geerts-Dimitriadou et al. 2011b; Liu et al. 2016; Yao et al. 2012). In addition, the presence of multiple bases with complementarity to the viral RNA 
template increases the usage of leader sequences as cap-donor (van Knippenberg et al. 2005), as is also the case for nuclear cap-snatching. While PAR has first been discovered and described for a Hantavirus (Garcin et al. 1995), it meanwhile has also been observed for many other cytoplasmic replicating, plant- and animal-infecting segmented NSVs (Yao et al. 2012). The optimal size of capped-RNA leaders varies and ranges between 11-18 nt for TSWV, 11-14 for La Crosse virus, and 12-20 nt for RSV, whereas the non-viral leader sequences are relatively short for Arenaviruses, with lengths of 1-7 nt (Barr 2007; Duijsings et al. 1999; Meyer and Southern 1993; Raju et al. 1990; Yao et al. 2012).

In general, the length of the capped leader is not longer than 20 nt for all NSVs, whether cytoplasmic or nuclear. Although speculative, differences in the length of snatched nonviral leader sequences may be caused by the steric conformation of the viral polymerase complex, in which the distance between the endonuclease domain and the cap-binding domain might determine the length of leaders.

Instead of a complex of three subunits like for influenza virus, the RdRp of the cytoplasmic replicating, segmented NSVs consists of one single, large polypeptide of about $250-450 \mathrm{kDa}$ (Fig. 1.3A). Structural studies of cytoplasmic-replicating bunyaviruses showed that the $\mathrm{N}$-terminal region of the RdRp protein possesses endonuclease activity that is essential for viral transcription via leader sequence cleavage (Reguera et al. 2010; Zhao et al. 2019), and structurally comparable to the influenza virus PA endonuclease. The enzymatic RNA polymerase activity is located in a central domain, and exhibits similarity to the influenza virus PB1 unit (Reguera et al. 2010). Whereas the influenza virus PB2 unit contains a capbinding domain, no structural (primary) sequence similarity is found with the C-terminal domain of the bunyavirus RdRp protein (Fig. 1.3B). On the other hand, studies on the Lassa mammarenavirus (LASV) and Rift vally fever virus (RVFV) RdRp/L protein have shown the presence of several aromatic and charged amino acids in the C-terminal region of the RdRp protein that could reflect a cap-binding domain (Gerlach et al. 2015). Recent studies to determine the structural conformation of RVFV L protein and a Reptarenavirus $L$ protein have revealed a structural resemblance of their C-terminal domains to the cap-binding domain of the Influenza virus PB2 protein (Gogrefe et al. 2019; Rosenthal et al. 2017). However, biochemical studies failed to confirm the binding activity of the cap-binding domain for the reptarenavirus $L$ protein, and only a low affinity of the RVFV L protein for 5' cap-structures (Gogrefe et al. 2019; Rosenthal et al. 2017; Vogel et al. 2019). As a consequence, it still remains an enigma as to whether the RdRps of the cytoplasmic replicating segmented NSVs 
recognize and bind to 5'capped-RNA molecules, or whether another protein is required for this, either alone or in concert with the RdRp.

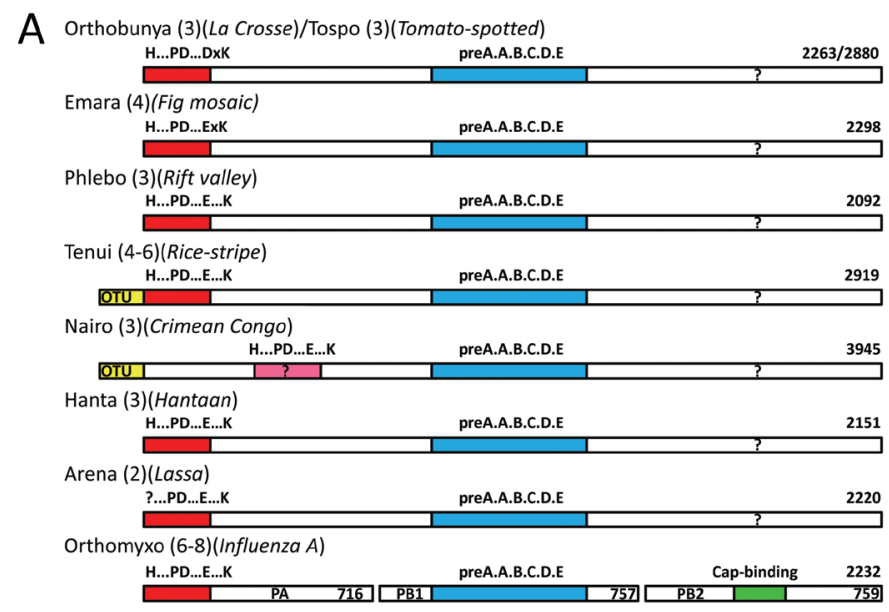

B

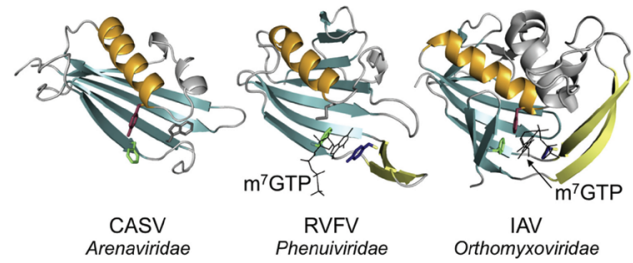

Figure 1.3 Comparison of Bunyaviruses and influenza virus $L$ protein. (A) Schematic diagram comparison of Bunyaviruses and influenza virus $L$ protein (Reguera et al. 2010). (B) Structural comparison of (putative) cap-binding domains of Bunyaviruses and influenza virus (Olschewski et al. 2020).

\section{A proposed role for the $\mathrm{N}$ protein in cap-structure recognition in cytoplasmic NSVs}

Interestingly, earlier studies on the Sin nombre hantavirus (SNV) have shown that its N protein exhibited a higher affinity to capped RNA when being offered a 1:1 mix of capped and uncapped RNA (Mir et al. 2010). Furthermore, for the Junin, Tacaribe, and Pichinde arenaviruses, their $\mathrm{N}$ protein has been shown to be able to interact with a 7-methylguanosine (cap) (Jeeva et al. 2017a; Jeeva et al. 2017b). Although no evidence has been reported for a direct interaction between the $\mathrm{N}$ protein of other viruses and $5^{\prime}$ capstructures, those of Crimean Congo haemorrhagic fever nairovirus (CCHFV) and the TSWV tospovirus enhance translation of mRNAs (Geerts-Dimitriadou et al. 2012; Jeeva et al. 2017a; Jeeva et al. 2017b). 


\section{Hypothesis on the source of the capped leader sequences for cytoplasmic NSVs}

Another unsolved issue remains the source of mRNAs from which the cytoplasmic replicating NSVs collect 5'-capped-RNA leader sequences to prime transcription of their viral RNA genome. Due to being confined to the cytoplasm it is likely that those viruses take 5' Capped-leader RNAs from an existing pool somewhere (and preferably concentrated) in the cytoplasm. The viroplasm induced upon viral infection is generally regarded the place where viral replication and transcription processes occur (Novoa et al. 2005)(Fig. 1.2). Whether the viroplasm is spatially linked to a cytoplasmic foci enriched in ((non)functional) mRNAs, thereby having direct access to a pool of 5'-capped RNA leader sequences remains an important question. While the SNV hantavirus N protein has affinity to 5'cap-structures, the protein also localizes specifically to RNA processing bodies (P bodies) (Mir et al. 2008b). The RVFV N protein has been reported to compete for the DCP2-accessible mRNA pool resident to $P$ bodies (Hopkins et al. 2013). P bodies are cytoplasmic mRNA granules which harbour enzymes such as Decapping enzyme 1(DCP1), Decapping enzyme 2(DCP2) and 5'3' Exoribonuclease 1(XRN1), involved in RNA surveillance, RNA quality control (RQC) and decay (Parker and Sheth 2007). Although studies on SNV and RVFV indicate an interplay with $P$ bodies as a source for capped-RNA leaders, it still remains to be analysed whether this applies and is generic for all cytoplasmic replicating NSVs.

So far it still has remained an intriguing question as to how and where the cytoplasmic replicating, segmented NSVs recognize 5'cap-structures of host cellular mRNAs for transcription initiation of the viral genomic RNA. The lack of evidence that undisputedly shows a role in binding a $5^{\prime}$ cap-structure for the RdRp of any given cytoplasmic replicating segmented NSV, and the observation that some N proteins either exhibit affinity to $5^{\prime}$ capstructures or enhance translation of mRNAs, has tempted us to investigate the idea whether the $\mathrm{N}$ protein may act functionally similar to the Influenza PB2 protein.

If binding to 5'cap-structures by the $\mathrm{N}$ protein is generic for all these cytoplasmic replicating, segmented NVSs, those N proteins, once expressed in a cell, would probably localize to cytoplasmic foci enriched for cellular mRNAs. On this point, studies performed with the SNV (hantavirus) N protein have already pointed towards $\mathrm{P}$ body as a source for cappedRNA leaders (Mir et al. 2008b). The conclusion was based on co-localization studies of the SNV nucleocapsid (N) protein with the P body marker Decapping 1 enzyme (DCP1) and the affinity of the $\mathrm{N}$ protein to $5^{\prime}$ cap-structures (Mir et al. 2008b). Additional support was 
provided by the observation that SNV seemed to prefer host transcripts that target to $\mathrm{P}$ bodies via the nonsense-mediated decay (NMD) pathway, a cellular surveillance mechanism that detects mRNA transcripts containing a premature translation termination codon (PTC) and targets these to the P bodies for degradation (Cheng and Mir 2012; Mir et al. 2008b). However, rates of cap-snatching/viral transcription when offering a PTC containing transcript was similar in normal and $P$ body deficient cells. This raised the question on the role of $P$ bodies as (first and sole?) source for capped RNA leaders to support cap snatching. Whether stress granules, another form of cytoplasmic foci containing "silenced" mRNAs, that share an intimate relation with $\mathrm{P}$ bodies, could present another source for these leaders has never been investigated, and the same holds true for $U$ bodies. $U$ bodies also associate with $P$ bodies and present RNP structures that contain uridine-rich, capped small nuclear (sn)RNAs that mature in the cytoplasm (Liu and Gall 2007), but finally localize in the nucleus where they play a key role in RNA Pol II complex-mediated pre-mRNA processing. Support for $U$ bodies as being an alternative source of leader sequences comes from two recent studies on influenza virus transcription where snRNAs presented a preferred source for capped-RNA leaders (Gu et al. 2015; Koppstein et al. 2015). However, whether U bodies can also play such a role in their cytoplasmic phase, as would be needed for bunyaviruses, is unknown.

\section{Scope of the thesis}

The studies described in this thesis are performed with emphasis on the plant-infecting bunyavirus TSWV and atempts to identify sources from which the cytoplasmic replicating, segmented NSVs steal capped-RNA leaders. Prior to experimental studies, Chapter 2 first provides a detailed overview on nuclear and cytoplasmic RNA granules and their observed roles in pro-/-antiviral RNA activities.

In order to identify the source of non-viral, host-derived capped-RNA leaders from viral mRNAs by a large-scale sequence analysis, an RNA-seq approach is implemented on TSWVinfected leaf material (Chapter 3 ). Viral reads are analysed for the presence of non-viral leader sequences at the $5^{\prime}$ end and data base searches are performed to find the host cells' RNA sources that serve as cap-donor. By analysis of samples taken at various times post infection, the dynamics of the viral infection in the host plant is simultaneously being analysed.

In Chapter 4, the TSWV N protein is localized in situ, in plant and animal systems, relative to cytoplasmic RNA granules, in specific $P$ bodies and stress granules (SGs). To indicate whether 


\section{Chapter 1}

the observed cellular localization of TSWV N relative to $\mathrm{P}$ bodies and SGs is generic for all segmented NSVs, the N proteins of Schmallenberg orthobunyavirus, SNV hantavirus, CCHFV nairovirus, but also those of the plant-infecting RSV phenuivirus, and European Mountain ash ringspot associated virus (EMARAV) Emaravirus, are included in the analysis. To indicate the role of $\mathrm{P}$ bodies and SGs in viral genome transcription/replication, cells are depleted from P body and/or SG elements and challenged with TSWV or SNV to subsequently determine the effect on viral titres.

Following and complementary to the RNA-seq analysis described in chapter 3 , a 5'RACE approach is taken to specifically clone the non-viral leader sequences of viral mRNAs (Chapter 5). Cloned leader sequences are compared and added to the data from RNAseq. Sequences are mapped to the Nicotiana genome and transcripts of the donor genes identified are investigated on their (putative) cellular distribution and links to cytoplasmic RNA granules.

To further support these studies, and to investigate whether there is a preference for cappedRNA leaders from mRNAs targeted to P body or SG, cap-snatching assays are performed on TSWV-infected leaf material that simultaneously has been offered an exogenous source of a functional mRNA, and a modified version (containing an additional marker nucleotide in its leader sequence) that complies with either the NMD pathway criteria to reach $P$ bodies or up to $\sim 7 \mathrm{~kb}$ in size to reach SG under stress conditions. The preference of the virus for $\mathrm{P}$ bodies and/or SGs as source for capped-RNA leaders is determined by cloning and nucleotide sequence determination of non-viral leader sequences, and the analysis for the presence or absence of the marker nucleotide from the exogenously applied mRNA in the used leader sequences.

Finally, in Chapter 6, all findings from this thesis research study are being discussed in light of the newly obtained knowledge. The role of viral proteins and the interplay with the cytoplasmic machinery is indicated and a perspective given on a cap-snatching model as a paradigm for all cytoplasmic replicating, plant- and animal-infecting segmented NSVs. 


\title{
Chapter 2
}

\section{Cellular RNA Hubs: Friends and Foes of Plant Viruses}

\author{
Min Xu, Magdalena J. Mazur, \\ Xiaorong Tao and Richard J. M. Kormelink
}
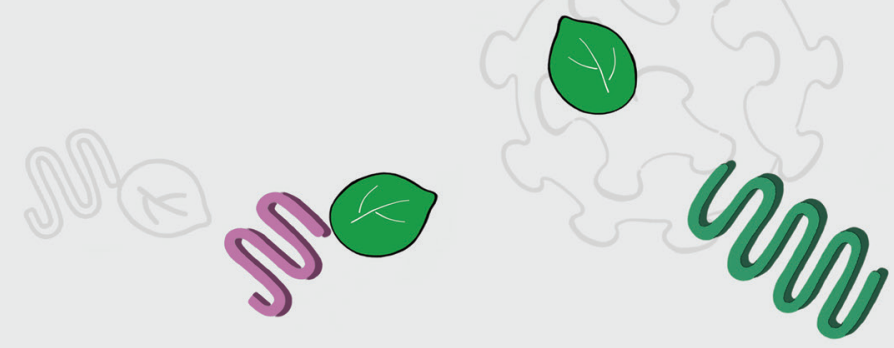

This chapter has been published in a slightly modified version as:

Min Xu, Magdalena J. Mazur, Xiaorong Tao and Richard Kormelink (2019). Cellular RNA Hubs: Friends and Foes of Plant Viruses. Mol Plant Microbe Interact, 2019: p. MPMI06190161FI. 


\section{Abstract}

RNA granules are dynamic cellular foci that are widely spread in eukaryotic cells and play essential roles in cell growth and development, immune and stress responses. Different types of granules can be distinguished, each with a specific function and playing a role in e.g. RNA transcription, modification, processing, decay, translation and arrest. By means of communication and exchange of (shared) components they form a large regulatory network in cells. Viruses have been reported to interact with one or more of these either cytoplasmic or nuclear granules, and act either pro-viral to enable and support viral infection and facilitate viral movement, or antiviral, protecting and/or clearing hosts from viral infection. This review describes an overview and recent progress on cytoplasmic and nuclear RNA granules and their interplay with virus infection, first in animal systems and as a prelude to the status and current developments on plant viruses, which have been less well studied on this so far. Most of those interactions serve to help viruses escape from the RNA gene regulation machinery and support their translation, replication and dissemination. 


\section{Introduction}

Eukaryotic cells have organized cellular RNA synthesis, their modification, translation and degradation in various cytoplasmic and nuclear RNA granules. The ones most well-known RNA granules are the cytoplasmic stress granules (SGs) and processing bodies (P bodies/PBs) and the nuclear Cajal bodies. RNA granules are defined as structures containing ribonucleoprotein complexes which play key roles in gene expression regulation, RNA turnover and storage/ degradation under different stress conditions (Anderson and Kedersha 2009). However, in the cytoplasm, besides PBs and SGs, also siRNA bodies, neuronal bodies (in neuron cells) and exosome bodies are present (White and Lloyd 2012). The nucleus comprises as well other distinct bodies, like the nucleolus, nuclear speckles, nuclear stress bodies, the transcription factories, the histone locus bodies, paraspeckles, animal/human-specific promyelocytic leukaemia bodies (PML bodies) and present only in plant cells- photobodies (Dundr and Misteli 2010; Morimoto and Boerkoel 2013). All these RNA granules do not function individually, but rather are part of finely tuned cellular regulatory network.

Viruses pose a stress condition on cells and trigger and/or modulate the formation of such RNA granules, often for their own benefit. For example, in response to Hepatitis C virus (HCV) not only assembly of SGs is induced but also the virus, by means of two viral replication proteins, interacts with SG component G3BP1 (GTPase activating protein (SH3 domain) binding protein 1), suggesting a direct (pro-viral) role of SGs in viral infection (Garaigorta et al., 2012; Li et al., 2013; (Poblete-Durán et al. 2016). During Rubella virus (RUBV) infection SG formation is induced to facilitate viral encapsidation (Matthews and Frey 2012; PobleteDurán et al. 2016). Although viral proteins often interact with SG components for their own benefits, as nicely described in several recent review papers (Pager et al. 2013; White and Lloyd 2012), SG may also act antiviral. In order to establish a viral infection under such condition, viruses must counter-defend host defence responses, by for instance inhibiting SG formation.

The development of Laser scanning Confocal Microscopy (LSCM) and fluorescence techniques has greatly boosted and facilitated visualization and studies on the subcellular granules and their relationships during the past two decades. The importance of RNA granules in cell development and their roles in viral infection to some extent is still elusive, and has attracted an increasing number of virologists to study the relationships between RNA granules and virus infection. Within the past decade numerous studies have been 
performed in animal virus cell systems and readers are referred to some good and detailed reviews on this for further reading (Beckham and Parker 2008; Reineke and Lloyd 2013a; Tsai and Lloyd 2014a; White and Lloyd 2012). Studies in plant virus host systems, however, are still more limited. In this review, present a brief overview on what is known from animal systems and the most recent developments, together with a summary (on commonalities and differences) and outlook on the current state of plant virus study. Irrespective of animalor plant viruses being involved, it is expected that knowledge on this and the identification of pro- and antiviral host factors on the long term may contribute to the development of strategies to combat viral diseases.

\section{RNA processing bodies (PBs), GW bodies and stress granules (SGs): Storage sites for "silenced" mRNA but distinct in their function and composition}

In animal and yeast: During the RNA life cycle various mechanisms regulate the level and rate of gene expression and RNA turnover. Once mRNAs are produced in the nucleus, they are exported into the cytoplasm and recruitment of the translation initiation complex to initiate protein synthesis. If not, (bulk) (m)RNAs face aggregation and storage in SGs, or assemble into PBs for further degradation (Kedersha et al. 2005). Although SGs and PBs are cytoplasmic structures with distinct functions in the mRNA triage, both structures share several proteins and components and coordinate during stress conditions, PBs are constitutively present while SGs can only be observed during stress conditions. In some occasions, PB and SG component proteins also function in other molecular processes such as splicing, signalling and development (Anderson and Kedersha 2008). SGs are formed by macromolecular assemblies stalling translation preinitiation factors in response to stress conditions (Anderson and Kedersha 2009). Under normal conditions, mRNAs are delivered from nucleus to cytoplasm. Following the binding of elF4E to the $5^{\prime}$ Cap structure, the translation initiation complex and ribosome are recruited and mRNAs engage in translation, ending up in polysomes when multiple ribosomes translate the mRNA at the same time (Moore 2005). In animal cells, when disassembly of polysomes is initiated by stress, T-cell intracellular antigen (TIA)-1 and TIA-1-related protein (TIAR) links to elF2 $\alpha$ phosphorylation which interferes with elF2 $\alpha$-GDP recycling and compromises the availability of elF $2 \alpha-G T P-$ Met-tRNAiMet during translation. Ribosomal translation is stalled, transcripts detach and release as circular polyadenylated mRNPs (Kedersha et al. 1999; Mclnerney et al. 2005). TIA-1, TIAR and other mRNA binding proteins including tristetraprolin (TTP), BRF1 RNA polymerase III transcription initiation factor subunit (BRF1), Fragile $X$ mental retardation 
protein (FMRP), Fragile X mental retardation syndrome-related protein 1 (FXR1), cytoplasmic polyadenylation element binding protein 1 (CPEB), G3BP, and survival motor neuron protein $(\mathrm{SMN})$ mediate primary aggregation of SGs. Interactions between these proteins promote secondary aggregation of mRNPs and assemble into microscopically visible SGs. Next, SGs integrate other aspects of cellular metabolism and deliver signals to downstream pathways. On the longer term, stored mRNAs may reinitiate in translation or become degraded (Anderson and Kedersha 2008).

$P$ bodies have a different function in RNA turnover and contain components of the mRNA decay machinery whose assembly is dependent on the pool of untranslated mRNA (Liu et al. 2005; Pillai et al. 2005; Teixeira 2005). Apart from mRNA turnover, $P$ bodies are also involved in 5'-3' RNA decay, RNA transportation and stabilization and the RNA interference pathway (Anderson and Kedersha 2009; Jakymiw et al. 2005; Moser et al. 2007). Several proteins are essential and unique to $P$ bodies which include decapping enzymes (DCP1/DCP2), activators of decapping (Dhh1p, Pat1p, Lsm1-7p), RNA helicase DDX6 and the 5' to 3' exonuclease Xrn1p, while $\mathrm{P}$ bodies generally lack translation factors (Eulalio et al. 2007; Ingelfinger et al. 2002; Lykke-Andersen 2002; van Dijk et al. 2002). In eukaryotes, massive mRNAs undergo decay by a pathway that is initiated by poly(A)-tail shortening. Deadenylation of mRNAs is a prerequisite of $\mathrm{P}$ body formation, and most mRNAs within $\mathrm{P}$ bodies lack (long) poly $(\mathrm{A})$ tails (Kulkarni et al. 2010).

In P bodies the $5^{\prime}$ cap structure is removed from these molecules by DCP2 (decapping enzyme 2) leading to the generation of mRNAs that expose a $5^{\prime}$ monophosphate and become further processed by XRN1, a cytoplasmic exoribonuclease for 5' to 3' mRNA-decay (Eulalio et al. 2007). Depletion of decapping co-activators like DCP1 and Lsm1-7 cause the loss of P bodies. Although (m)RNAs mostly end up in P bodies for turnover/decay, studies have shown that mRNAs can also go back into translation (Coller and Parker 2005; Sheth and Parker 2003; Tharun et al. 2005). mRNAs that experience re-initiation first go to SGs before eventually entering polysomes (Stoecklin and Kedersha 2013). During mRNA translation, SGs and P bodies cooperate structures to maintain proper translation rates (Parker and Sheth 2007).

In both mammalian cells and yeast cells, $\mathrm{P}$ bodies and SGs have been observed to form docking stages (Brengues 2005; Eulalio et al. 2007), during which RNA and protein components exchange. This also explains why some proteins were localized in both $\mathrm{P}$ bodies and $\mathrm{SGs}$. $\mathrm{P}$ bodies contain a 54-kilodalton D-E-A-D box protein (RCK/p54), CPEB, 5'-3' Exoribonuclease 1 (XRN1), elF4E, 
Fas-activated serine/threonine phosphoprotein (FAST) and TTP proteins, which will relocate to SGs under stress. Conversely, TIA-1 and TIAR proteins are known to predominantly exist in SGs but have also been found in P bodies (Eulalio et al. 2007). For this reason, marker proteins that specifically localize to only one of those granules are used to distinguish between PBs and SGs during cell biology studies. Translation factors such as elF3b, elF4A and eIF4G, and RBPs such as PABP and G3BP can be used as specific markers for SGs, while components of the cytoplasmic RNA degradation machinery like DCP2, DCP1 or Hedls serve as reliable marker proteins for $P$ bodies (Stoecklin and Kedersha 2013). Under different stress conditions, cells that were co-transfected with RFP-DCP1a (P body marker) and GFPTIA-1 (SG marker) show frequent association of SGs with one or more P bodies. TTP and BRF1 proteins, which are RNA-binding proteins that promote mRNA decay, regulate the dynamic interactions between SGs and P bodies (Kedersha et al. 2005).

Besides deadenylation of mRNA, organisms have additional pathways that remove RNAs away from active translation and finally towards $P$ bodies for degradation. The most wellknown are the nonsense-mediated decay (NMD) pathway and the miRNA- and siRNA mediated gene silencing pathways. Although these pathways involve a different process to determine the fate of their target, based on mRNP dynamics, they rely on the downstream $P$ bodies for degradation.

The NMD pathway recognizes mRNAs with aberrant termination codons and targets them into $\mathrm{P}$ bodies. Aberrant termination codons have multiple features like natural premature termination codons (PTCs), the nucleotide immediately following the stop codon, and the sequences, length, and associated proteins of $3^{\prime}$ UTR. These features may result in different translation termination and/or decay of mRNAs (Shyu et al. 2008). In yeast, three conserved proteins, UPF1 to -3 (standing for ATP-dependent RNA helicase upstream frameshift-1 to -3), are functional in the recognition of PTCS of which UPF1 is the most abundant/prominent one, and is sufficient for targeting mRNAs to P bodies, while UPF2 and -3 are downstream triggers for $P$ body targeting (Eulalio et al. 2007; Sheth and Parker 2006). Serine/threonineprotein kinases SMG1 and SMG5-7 are also conserved NMD effectors that localize to $P$ bodies (Fukuhara et al. 2005; Unterholzner and Izaurralde 2004). Furthermore, Human SMG7 recruits the surveillance-complex protein UPF1 and SMG5 to P bodies (Unterholzner and Izaurralde 2004). The NMD pathway mainly serves to recognize and eliminate PTC-containing mRNAs to prevent the production of aberrant and potentially toxic proteins. However, and interestingly, UPF1 is also able to target normal mRNAs to P bodies (Sheth and Parker 2006) 
and a growing amount of evidence indicates a role of UPF1 in various other RNA decay pathways and that it also can function as a E3-Ubiquitin ligase to play a role in protein decay (Feng et al. 2017; Kim and Maquat 2019).

MicroRNAs (miRNAs) and small-interfering RNAs (siRNAs) are hallmarks of RNA interference (RNAi)/RNA silencing and consist of (endogenous) RNA duplex molecules 20-24 nucleotides (in animals, 21) in size that are processed from dsRNA molecules by RNaselll-like enzymes called Dicer. Both types of small RNAs play a major role in the regulation of gene expression via post-transcriptional and transcriptional gene silencing. MiRNAs originate from noncoding transcripts that fold in relatively short imperfect dsRNA hairpin loop structures (pri-miRNA) and are processed into pre-miRNAs. These are transported from the nucleus into the cytoplasm for further maturation into miRNAs. SiRNAs are produced from relatively long perfect dsRNA molecules. Although the biogenesis of miRNAs and siRNAs differ, they are structurally and functional similar. From these duplex molecules one strand associates with Argonaut protein (AGO) to activate an RNA-induced silencing complex (RISC). While miRNAloaded RISC complexes repress translation and/or trigger degradation of target mRNAs (Eulalio et al. 2008), siRNAs strictly target, cleavage and degrade the mRNAs with sequence complementarity (Hannon 2002).

Proteins that govern miRNA-mediated silencing, such as GW-182/Gw and AGO1, are often associated with P bodies of higher eukaryotic organisms (Jakymiw et al. 2005; Pillai et al. 2005). For this reason, GW containing-bodies (also referred to as GW-bodies) have long been thought to be similar to $\mathrm{P}$ bodies. However, several reports have indicated that GW-bodies have different dynamics to $P$ bodies. Like $P$ bodies, GW-bodies contain factors required for RNA decapping and decay, like DCP2, LSm-4 and Xrn1 (Behm-Ansmant et al. 2006; Eystathioy et al. 2003), but, in contrast, they have been observed to lack decapping factors like e.g. DCP1 and Me31B, and have also been observed in the nucleus (Patel et al. 2016).

GW-182 is a scaffold of protein complexes involved in miRNA-mediated silencing of RNA targets. GW-182 contains an N terminal domain with GW/WG motifs to enable binding to Argonaute (AGO) proteins, a ubiquitin-associated domain (UBA) and a silencing domain that contains an RNA recognition motif (RRM) and CCR4-NOT interacting motifs. GW-182 associates with a unique subset of mRNAs (Eystathioy et al. 2002). Although the mechanism on how GW-182 causes translational repression and miRNA-mediated target degradation is still not yet fully understood, the protein interacts with a specific subset of AGO proteins 
involved in the miRNA pathway, the cytoplasmic poly(A)-binding protein (PABP), PAN2-AND3 and CCR4-NOT deadenylase complexes (Behm-Ansmant et al. 2006). The W motifs of GW182 recruit deadenylase complexes and depends on CCR4-NOT for poly(A)- mRNA repression but no direct link has been observed between recruitment of CCR4-NOT and mRNA repression (Chekulaeva et al. 2011). In animals, CAF1 and/or NOT regulate the expression of most of AGO1 targets and indicate that deadenylation by the CAF1-CCR4-NOT1 complex is a widespread effect of miRNA regulation (Eulalio et al. 2009). miRNA-mediated mRNA degradation involves alteration of mRNP composition and/or conformation, and does not result from direct interference with the binding and function of ribosomal units to mRNAs. While overexpression of DCP1a and G3BP1 is observed to induce P bodies and SGs, such induction of granules inhibits protein synthesis, while induction granules by GW-182 do not (Wang et al. 2017b). SiRNAs/miRNAs can function in the absence of detectable P bodies and their accumulation together with Argonaut proteins and mRNA targets in GW bodies is the consequence, rather than the cause of silencing (Pauley et al. 2006). Furthermore, in contrast to miRNAs, siRNAs may affect mRNA metabolism and reduce the amount of mRNA directed to GW bodies, and thereby could lead to the disappearance of GW bodies (Serman et al. 2007). Disruption of GW bodies impairs RNA interference in mammals(Jakymiw et al. 2005).

Altogether, this supports the idea that GW bodies are (functionally) distinct from P bodies, and likely play a role as repository for translationally silenced RNAs, but not in active translational silencing (Patel et al. 2016). Another distinct and unique feature for GW-182 is the observation that GW bodies, in contrast to P bodies and SG, have also been found in the nucleus.

In plants: It is generally believed that P bodies and SG are evolutionary conserved among eukaryotes and in plants to resemble structurally and functionally to those from animal/ yeast systems as described above. Although less research has been performed in plants, studies on these structures slowly unveil their roles in plant cells with essential roles in plant development and immune response (Bhullar et al. 2017; Dong et al. 2016; Meteignier et al. 2016; Xu and Chua 2011). Tobacco plant $P$ bodies play a role in reprogramming mature cells and re-initiation of the cell division cycle (Bhullar et al. 2017). In Nicotiana benthamiana, responses of dominant resistance ( $R$ ) genes from the class NB-LRR induce dramatic increase in the biogenesis of $\mathrm{P}$ bodies (Meteignier et al. 2016). Several homologs have been identified of animal proteins with a function in RNA granule formation and gene expression regulation, sometimes differently named though. 
In Arabidopsis thaliana (At), the major components of the decappping complex are DCP1, DCP2 and Varicose (VCS), proteins which present the homologs of Dcp1, Dcp2 and HEDLS/ GE-1 protein from animal systems (Weber et al. 2008; Xu et al. 2006). Later, AtDCP5 was identified as another component that complexes to Dcp $1 / 2$ and is required for decapping, translational repression and $\mathrm{P}$ body formation ( $\mathrm{Xu}$ and Chua 2009b). The triple RNA recognition motif (RRM) oligouridylate binding protein 1 (UBP1) protein exhibits highest amino acid similarity to the animal SG component TIA1/Rs. Additional triple RRM proteins with similarity to TIA1 are RNA-binding proteins 46 and 47 (RBP45/47) and PAB protein. During a stress response of hypoxia in Arabidopsis, UBP1C has been observed to dynamically and reversibly aggregate into cytoplasmic granules that contain poly $(A)+R N A$ and $P A B P$, and are referred to as UBP1 SGs (Sorenson and Bailey-Serres 2014). UBP1 likely functions in the same manner as mammalian TIA-1 in the sequestration of mRNA in SGs. Like TIA1, SGs are formed upon impairment of translation by phosphorylated elF $2 \alpha$. The formation of UBP1 SG in Arabidopsis seems to be energy related, and is promoted by e.g. arsenite and KCN (Sorenson and Bailey-Serres 2014). Recently, a plant homolog of SG-component G3BP has been cloned from Arabidopsis (Krapp et al. 2017). Under stress conditions, AtG3BP appears in a granular phenotype and co-localizes with TZF1, which is a protein that co-localizes with SGs and PBs components and binds both DNA and RNA in mammalians cells (Brodersen et al. 2008; Lykke-Andersen and Wagner 2005; Pomeranz et al. 2010). Cycloheximide treatments followed by heat stress abolishes AtG3BP granule formation, similar to what has been shown with UBP1 granules.

Besides the core protein homologs of $\mathrm{P}$ bodies and SG, more proteins have been identified in plants implicating a role in these structures. Tudor Staphylococcal Nuclease (TSN or Tudor-SN; also known as SND1) is a protein that is indispensable in stress tolerance. TSN1 and TSN2 co-localize with SGs in A. thaliana protoplasts and associate stably with SGs and P bodies (Gutierrez-Beltran et al. 2015). Angustifolia (AN), a plant homolog of CtBP/BARS, co-localizes with several mRNP granule markers to SGs and regulates their formation in a stress dependent manner (Bhasin and Hulskamp 2017). vascular plant one-zinc finger protein (VOZ2) co-localizes with both SGs and P bodies under heat stress condition and functions as a transcriptional repressor of DREB2A in Arabidopsis. In contrast to the situation in mammals where the movement of $P$ bodies requires microtubuli, in plants, the movement of $\mathrm{P}$ bodies depends on actin through the binding of AtDCP1 to myosin XI-K (Steffens et al. 2014). 
UPF1, the key factor for the assembly of the NMD core complex, is conserved across all eukaryotes and is also found in plants. A recent study (2018) in Arabidopsis revealed that more than $50 \%$ of the proteins that interact and co-localize with UPF1, can be co-purified with DCP5 (Chicois et al. 2018). In addition, three Arabidopsis homologs of the DEAD-box helicase 6 (DDX6), terminal nucleotidyl transferase, ribonucleases and RNA helicases RH6, $\mathrm{RH} 8$ and $\mathrm{RH} 12$, , are required for $\mathrm{P}$ body formation in animals (Chicois et al. 2018). UPF1 also co-localizes with typical P body components such as LSM14A and DCP5, which indicates that also in plants RNA degradation and translational repression interact with each other extensively.

GW-182 is an important element for GW bodies formation in animals and insects and it is involved in miRNA-mediated silencing of RNA targets, while no homologs of GW-182 have been found so far in plants and fungi (Behm-Ansmant et al. 2006; Braun et al. 2013; Eulalio et al. 2009). However, proteins are found in plants containing GW-repeats that interact with AGOs in a similar manner to GW-182 proteins. In A. thaliana those GW-repeats are found in e.g. NRPE1, a subunit of polymerase IV (involves in transcription of noncoding RNAs that are required for transcriptional gene silencing via the RNA-directed DNA methylation (RdDM) pathway, e.g. of transposable elements (Zhou and Law 2015), and SPT5-like transcription elongation factor. Both proteins are known to interact with AGO4 (Bies-Etheve et al. 2009; Lahmy et al. 2009), which is required to mediate transcriptional gene silencing in plants. Another protein containing GW-repeats is Silencing Defective 3 (SDE3), which has a putative RNA helicase that is the homolog of the mammalian MoV10 helicase, binds to AGO proteins and assists in the amplification of post-transcriptional gene silencing by RNA-dependent RNA polymerases 6 (RDR6, see below) (Garcia et al. 2012).

In contrast to the absence of typical GW bodies, plant cells contain siRNA bodies that are required for the amplification of RNAi, which is absent from animal cells. This process involves host-encoded RDRs. In A. thaliana six RDRs are found, of which RDR1, -2 and -6 have a well-established role in the amplification of RNAi amplification. RDR3, -4 and -5 have not been characterized with any function yet, although recently a homolog from tomato has been shown to involves in enhancing transcriptional gene silencing (Butterbach et al. 2014). Aberrant RNAs, resulting from activated RISC-Argonaut cleavage may enter $\mathrm{P}$ bodies for degradation, but can also be converted by RDRs into dsRNA and are subsequently processed into a population of secondary siRNAs. This process occurs in siRNA bodies, cytoplasmic granules which contain suppressor of gene silencing 3 and RDR6 (also named as SGS3/RDR6- 
bodies) and are different from $P$ bodies (Kumakura et al. 2009). P bodies and siRNA bodies both compete for the same RNA substrate. When P bodies are functionality compromised, aberrant RNAs may enter siRNA bodies, leading to a higher production of secondary siRNAs and concomitant stronger RNAi response. Conversely, a knock down of RDR6 leads to a reduced RNAi response and a stronger induction of $P$ bodies and degradation of aberrant RNAs (Martinez de Alba et al. 2015; Thran et al. 2012; Tsuzuki et al. 2017).

In plants siRNA or miRNA associate with Argonaut proteins and target mRNA to accomplish endonucleolytic cleavage. This process is catalysed by the C-terminal PIWI domain of Argonaut proteins. Afterwards, small fragments of RNA products enter the general mRNAdecay pathway (Lingel and Sattler 2005; Orban and Izaurralde 2005). In Arabidopsis, mRNA cleavage products experience 3' removal by XRN4 proteins (Souret et al. 2004).

\section{Nuclear RNA granules}

The nucleus is the predominant compartment for cell RNA processing and metabolism, processes that are contained within nuclear RNA granule structures. So far, several different nuclear RNA granules have been identified, and among the most prominent are nucleoli, Cajal bodies (CBs), spliceosomes, speckles and dicing bodies. They are nuclear condensation centres for RNA molecules and proteins that are involved in processes like RNA splicing, ribosomal RNA ( $r R N A$ ) transcription, small nuclear ribonucleoproteins (snRNP) biogenesis and maturation, mi/siRNA-mediated gene silencing and stress responses. Nuclear RNA granules are highly dynamic, and their formation can be a result of external stimuli, like various stress conditions (Boulon et al. 2010).

The nucleolus is the biggest and the most prominent compartment of the nucleus. This membranelles organelle is organized into the fibrillar centre (FC), the dense fibrillar component (DFC), and the granular component (GC). It primarily serves as a factory for ribosomal RNA (rRNA) synthesis by RNA polymerase I transcription, rRNA processing and ribosome assembly(Stępiński 2014). However, the proteins involved in many other aspects of molecular cell biology, such as cell cycle regulation, development, telomerase activity, gene silencing, and (a)biotic stress signalling, suggests further roles for the nucleolus (Dubois and Boisvert 2016; Pendle et al. 2005). One of these processes identified that links nucleolus and cytoplasmic $P$ bodies is the mRNA quality control pathway by NMD. While the NMD pathway in animal cells is associated with cytoplasmic $P$ bodies, the presence of 
aberrant mRNA transcripts and NMD proteins UPF2,-3 and exon joining complex (EJC) in the nucleoli of plant cells indicates their involvement in NMD as well (Pendle et al. 2005; Sang Hyon Kim and and Michael Taliansky 2009).

Another type of most well studied nuclear granules are the Cajal bodies (CBs), also known as coiled bodies. CBs are very dynamic structures; the size and the number of the CBs depend on the type, cycle stage and transcriptional activity of the cell (Boudonck et al. 1998). They translocate within the nucleus and locate in the nucleoplasm and in the nucleolar periphery, but also can enter the nucleolus (Boudonck et al. 1999). Nucleolus and CBs not only are physically but also functionally connected. Both structures share structurally important proteins like fibrillarin or coilin, an essential scaffold protein for CB formation (Collier et al. 2006; Trinkle-Mulcahy and Sleeman 2016). Also, they participate in the assembly and processing of small nucleolar ribonucleoproteins (snoRNPs) and formation of spliceosomal snRNPs, core particles that control pre-mRNA splicing (Trinkle-Mulcahy and Sleeman 2017).

One main process that is intrinsically controlled by $\mathrm{CBs}$ is the formation of spliceosomal snRNPs, RNA-protein complexes that bind unmodified pre-mRNA to form a spliceosome. Therefore, CBs contain a number of proteins vital for splicing of pre-mRNA (such as the survival of motor neuron (SMN) protein, Sm or Gemin proteins, well characterized in animal cells) (Love et al. 2017). Newly transcribed uridine-rich U1, U2, U4, and U5 snRNPs are exported to the cytoplasm and together with the core Sm proteins assemble into SMN protein complexes. Most $U$ snRNPs assemble in the cytoplasm and spatially organize in the cytoplasm in discrete structures, which also have been referred to as $U$ bodies. $U$ bodies consistently associate to $\mathrm{P}$ bodies and disruption of the latter affects $\mathrm{U}$ body organization, indicating that $\mathrm{P}$ bodies and $\mathrm{U}$ bodies cooperate in the regulating aspects of snRNP metabolism (Liu and Gall 2007).

Evidence is accumulating that besides a major role in RNA metabolism and snRPN biogenesis, nucleolus-associated CBs also play a role in RNA silencing by siRNA and miRNA processing. In Arabidopsis, a pathway generating $24 \mathrm{nt}$ siRNAs is responsible for RdDM and transcriptional silencing, involving a number of proteins, like RNA polymerase IV (Pol IV), RDR2, Dicer-like 3 (DCL3), and Argonaute 4 (AGO4). Interestingly, RDR2, DCL3 and AGO4 are localized with one another in nucleolus-associated loci, which also colocalize with CB markers (Li et al. 2006; Pontes et al. 2006). Moreover, two major proteins involved in the miRNA pathway, DCL1 and Hyponastic leaves 1(HYL1) (Kurihara et al. 2006), were also found in these DCL3- 
containing siRNA foci, indicating that CBs emerge as siRNA and miRNA processing centres (Fujioka et al. 2007; Pontes and Pikaard 2008). It is, however, interesting to note that the nucleolar periphery-localized foci of DCL1 and HYL1 were previously referred to as dicing bodies, distinct from CBs as they lack coilin (Fang and Spector 2007; Song et al. 2007).

Besides the nucleolus and $\mathrm{CB}$, few other nuclear bodies are reported, some with similarity to $C B$. The Histone locus body is a subnuclear body that resembles CB physically, by shape and size, and functionally, as they share proteins and RNA molecules (Nizami et al. 2010a). Although a substantial part of HLB contains coilin, in contrast to CB, HLB does not require coilin for its assembly (Nizami et al. 2010b). HLBs are known to be involved in the processing of histone pre-mRNAs (Nizami et al. 2010b).

There are other nuclear bodies that are shown to accumulate RNA of which the precise function remains to be determined (Mao et al. 2011). Nuclear speckles (NSs) present sites for storage and modification of splicing factors, which indicates their role in the coordination of nuclear gene expression regulation. This is supported by the observation that RNA polymerase II transcription occurs nearby NSs (Galganski et al. 2017). Paraspeckles are nuclear bodies that form upon stress and consists of the long noncoding NEAT (nuclear paraspeckle assembly transcript 1) RNA scaffold associated with a large amount of RNA binding proteins. Paraspeckles, therefore have been suggested to act as sequestration "sponge" for proteins (Bond and Fox 2009; Fox et al. 2018). Polycomb group bodies (PcG bodies) present a-typical nuclear bodies that show up at target genes to modulate their activity by epigenetic regulation of their transcription in eukaryotes. PcG proteins form multi-protein complexes, known as Polycomb repressive complex 1 (PRC1) and Polycomb repressive complex 2 (PRC2) that localize to PcG bodies (del Olmo et al. 2016; Mao et al. 2011; Pico et al. 2015).

\section{RNA granules and the interplay with viruses}

There are many excellent reviews that describe the interplay between different cytoplasmic RNA granules and viral infection in animal or yeast systems, either involving immunity responses to inhibit viral accumulation or facilitate viral proteins to accomplish their replication, translation and movement (Beckham and Parker 2008; Poblete-Durán et al. 2016; Reineke and Lloyd 2013a; Tsai and Lloyd 2014a; White and Lloyd 2012). Separate papers also have described the involvement of nuclear bodies. Studies on plant virus-granule interaction just more recently emerge and reviews on cytoplasmic granules and nucleus 
have separately appeared (Kim et al. 2004; Li et al. 2018b; Mäkinen et al. 2017). Without becoming too extensive, and to give a glimpse on the diversity of interplay, a few examples are given for animal viruses, and readers are referred to some nice reviews for further reading (Lloyd 2015, 2016; Onomoto et al. 2014; Poblete-Durán et al. 2016; Poblete-Duran et al. 2016), prior to a more extensive description on the recent progress and current status with plant viruses.

During viral infection of animal cells SG are often reported to act either antiviral or proviral. Upon virus infection, interferon stimulated gene (ISG) products like PKR, RNA-sensing receptors like RIG-I and MDA5, RNase L and OAS have been observed to localize in SGs (Hebner and Laimins 2006; Okonski and Samuel 2013; Onomoto et al. 2012), but are also found in PB (Hebner and Laimins 2006). Modulation of SG and PB therefor may promote viral replication and suppress stress responses/antiviral defence. E.g. members of the Togaviridae (like Semliki forest virus (SFV) and Chikungunya virus (CHIKV)) and Flaviviridae (West Nile (WN) virus, Zikavirus (ZIKV)) encode nonstructural proteins that sequester G3BP and TIA-1/TIAR resp., thereby preventing SG formation and promoting viral replication (Fros et al. 2012; Li et al. 2002a; Panas et al. 2012). Later on, G3BP was demonstrated to also play a pro-viral role in CHIKV replication (Scholte et al. 2015). The cellular NMD pathway restricts ZIKV infection, and the viral capsid protein has been shown to interact with UPF1 to target the protein for proteasomal degradation and thereby inhibit the NMD pathway (Fontaine et al. 2018). EJC has a role in NMD and both act antiviral to WNV. The EJC protein RBM8A directly binds WNV RNA, but the virus is able to counteract NMD and its capsid protein interferes with EJC function and localization (Li et al. 2019b).

Also the nuclear replicating Influenza virus inhibits SG formation during viral infection, via inhibition of PKR activity by the NS1 protein (Khaperskyy et al. 2012). Furthermore, the Influenza nucleoprotein (NP) and/or viral ribonucleoproteins (RNPs) have been observed to localize near or at nuclear CB and promyelocytic leukemia protein nuclear bodies (PML NBs) (Hofer et al. 2017), as well as cytoplasmic P bodies (Li et al. 2019a). In the latter case, viral RNPs interacted with the antiviral protein MoV10, a UPF-1 like RNA helicase that facilitates UPF1-mediated RNA degradation, likely for degradation of viral RNA. MoV10 normally localizes to $P$ bodies and SG where it associates to the RISC complex, but is also inhibited by the Influenza NS1 protein (Li et al. 2019). In contrast to Influenza virus, Vesicular stomatitis virus (VSV) induces the phosphorylation of elF $2 \alpha$ and promotes the assembly of SG-like particles that also contain viral replication proteins and RNA, indicating that these structures 
play a role in the replication cycle (Dinh et al. 2013). The nucleocapsid protein (NP) of Sin nombre hantavirus (SNV) has been shown to co-localize to $\mathrm{P}$ bodies from where the virus is postulated to use host cellular mRNAs containing a premature stop codon for cap-snatching to initiate viral genome transcription (Mir et al. 2008a).

Also plant viruses are reported to interplay with cytoplasmic or nuclear RNA condensations. However, the reason for this interplay sometimes still remains elusive. In Fig. 2.1, a schematic overview is presented on the regulatory network of different granules and the interplay with viral proteins/infections. Table 2.1 overviews reported studies of plant viral proteins and interplay with cytoplasmic and nuclear granules.

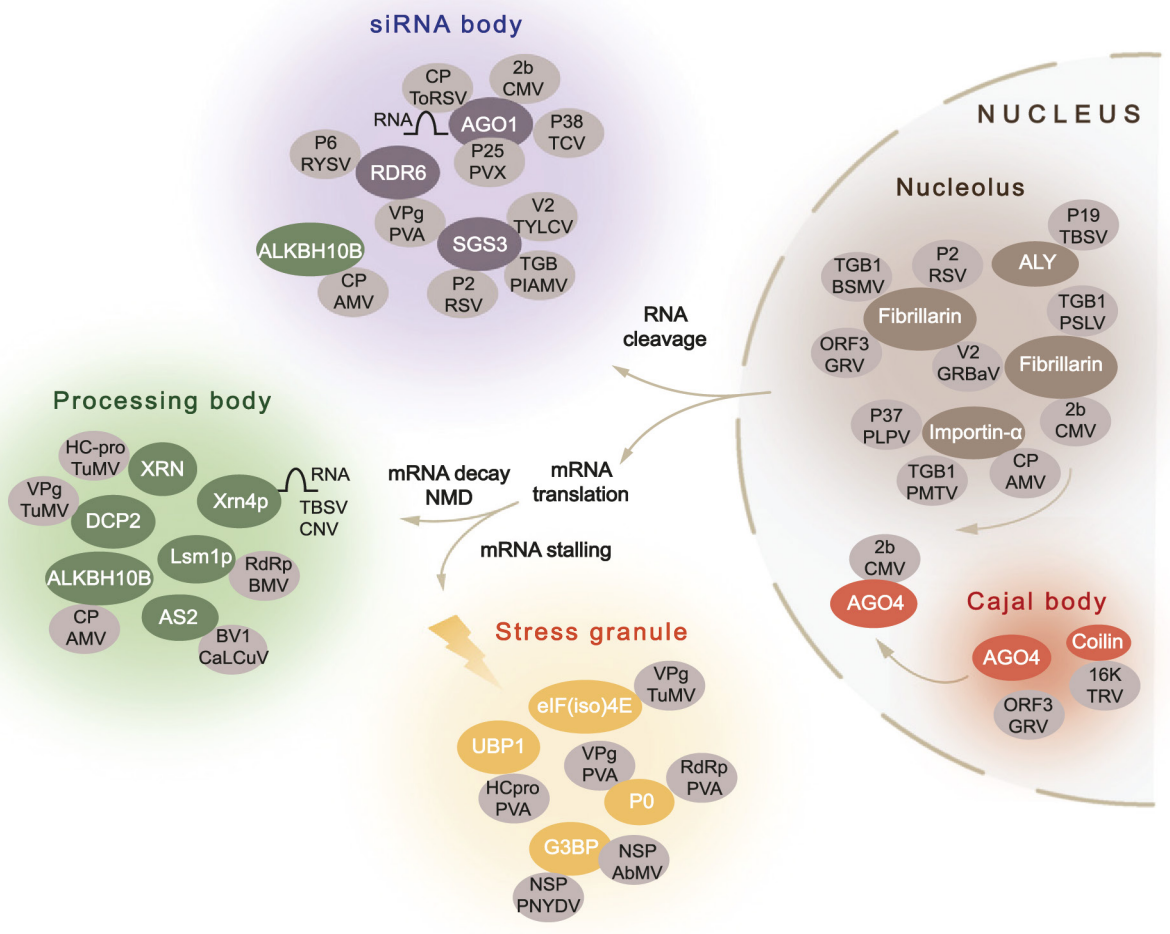

Figure 2.1 Schematic diagram on cellular RNA regulatory network hubs and targeting by plant viral proteins. Only viral proteins or RNA that have been identified to interact with proteins from cellular RNA granules are shown. 


\section{Chapter 2}

Table 2.1 Interactions reported on plant RNA granule (and related) components and plant viral proteins, and potential function.

\begin{tabular}{|c|c|c|c|c|}
\hline $\begin{array}{l}\text { Nuclear body } \\
\text { Components }\end{array}$ & Viruses & $\begin{array}{c}\text { Viral } \\
\text { components }\end{array}$ & Phenotypes & References \\
\hline Fibrillarin2 & $\begin{array}{c}\text { Grapevine red blotch- } \\
\text { associated virus } \\
\text { (GRBaV) }\end{array}$ & V2 & $\begin{array}{l}\text { Movement of the viral } \\
\text { genome from the nucleus } \\
\text { to neighboring cells }\end{array}$ & (Guo et al. 2015) \\
\hline Importin- $\alpha$ & $\begin{array}{c}\text { Potato mop-top virus } \\
\text { (PMTV) }\end{array}$ & TGB1 & $\begin{array}{c}\text { Virus systemic movement } \\
\text { and systematic } \\
\text { accumulation }\end{array}$ & $\begin{array}{c}\text { (Lukhovitskaya et al. } \\
\text { 2015) }\end{array}$ \\
\hline Fibrillarin2 & $\begin{array}{c}\text { Barley stripe mosaic } \\
\text { virus (BSMV) }\end{array}$ & TGB1 & Cell-to-cell movement & (Li et al. 2018b) \\
\hline coilin & $\begin{array}{l}\text { Groundnut rosette } \\
\text { virus (GRV) }\end{array}$ & ORF3 & $\begin{array}{c}\text { Formation of viral } \\
\text { ribonucleoprotein (RNP) } \\
\text { particles and long-distance } \\
\text { movement }\end{array}$ & $\begin{array}{l}\text { (Canetta et al. 2008; } \\
\text { Kim et al. 2007a; } \\
\text { Kim et al. 2007b) }\end{array}$ \\
\hline Importin- $\alpha$ & $\begin{array}{l}\text { Alfalfa mosaic virus } \\
\text { (AMV)/ (PLRV)/ } \\
\text { Satellite panicum } \\
\text { mosaic virus (SPMV) }\end{array}$ & $\mathrm{CP}$ & $\begin{array}{l}\text { Nucleolar/Cytoplasmic } \\
\text { shuttling and virus } \\
\text { replication and translation }\end{array}$ & $\begin{array}{l}\text { (Herranz et al. 2012; } \\
\text { Kalinina et al. 2018; } \\
\text { Taliansky et al. 2010) }\end{array}$ \\
\hline Fibrillarin & $\begin{array}{l}\text { Poa semilatent virus } \\
\text { (PSLV) }\end{array}$ & TGB1 & Unclear & $\begin{array}{c}\text { (Semashko et al. } \\
\text { 2012) }\end{array}$ \\
\hline Fibrillarin & $\begin{array}{c}\text { Rice Stripe Tenuivirus } \\
\text { (RSV) }\end{array}$ & p2 & $\begin{array}{l}\text { Promotion of virus systemic } \\
\text { movement }\end{array}$ & (Zheng et al. 2015) \\
\hline Fibrillarin & Bamboo mosaic virus & & $\begin{array}{l}\text { cell-to-cell movement and } \\
\text { systemic infection }\end{array}$ & $\begin{array}{l}\text { (Chang et al. 2016; } \\
\text { Zheng et al. 2015) }\end{array}$ \\
\hline fibrillarin & $\begin{array}{c}\text { Cucumber mosaic } \\
\text { virus (CMV) }\end{array}$ & $2 b$ & $\begin{array}{l}\text { Systemic symptom } \\
\text { development }\end{array}$ & (Du et al. 2014). \\
\hline importin & $\begin{array}{c}\text { Tombusvirus/ } \\
\text { Pelargonium line } \\
\text { pattern virus (PLPV) }\end{array}$ & P37 & infectivity & $\begin{array}{c}\text { (Perez-Canamas and } \\
\text { Hernandez 2018) }\end{array}$ \\
\hline ALY4 & $\begin{array}{l}\text { Tomato yellow leaf } \\
\text { curl virus (TYLCV) }\end{array}$ & $\mathrm{CP}$ & infectivity & (Wang et al. 2017a) \\
\hline
\end{tabular}




\begin{tabular}{|c|c|c|c|c|}
\hline $\begin{array}{l}\text { Cytoplasmic } \\
\text { granule } \\
\text { components }\end{array}$ & Viruses & $\begin{array}{c}\text { Viral } \\
\text { components }\end{array}$ & Phenotypes & References \\
\hline $\begin{array}{l}\text { ASYMMETRIC } \\
\text { LEAVES } 2 \text { (AS2) }\end{array}$ & $\begin{array}{l}\text { Cabbage leaf curl } \\
\text { virus (CaLCuV) }\end{array}$ & BV1 & $\begin{array}{c}\text { Activate DCP2 decapping } \\
\text { and increase viral } \\
\text { accumulation }\end{array}$ & $\begin{array}{l}\text { (Ghoshal and } \\
\text { Sanfacon 2014; Ye } \\
\text { et al. 2015) }\end{array}$ \\
\hline AGO1 & Tomato ringspot virus & RNAs & Symptom recovery & $\begin{array}{c}\text { (Ghoshal and } \\
\text { Sanfacon 2014) }\end{array}$ \\
\hline AGO1 & Turnip crinkle virus & P38 & $\begin{array}{l}\text { Mimic AGO-binding GW- } \\
\text { repeat proteins }\end{array}$ & (Azevedo et al. 2010) \\
\hline AGO1 & $\begin{array}{c}\text { Potato virus } X / \text { Beet } \\
\text { western yellows } \\
\text { virus/Tomato ringspot } \\
\text { virus }\end{array}$ & $\begin{array}{l}\mathrm{P} 25 / \mathrm{P0} / \text { coat } \\
\text { protein }\end{array}$ & AGO1 degradation & $\begin{array}{l}\text { (Baumberger et al. } \\
\text { 2007; Bortolamiol } \\
\text { et al. 2007; Chiu et } \\
\text { al. 2010) }\end{array}$ \\
\hline $\begin{array}{c}5^{\prime}-3^{\prime} \\
\text { exoribonuclease } \\
\text { AtXrn4p }\end{array}$ & $\begin{array}{c}\text { Tomato bushy stunt } \\
\text { virus (TBSV) }\end{array}$ & Viral RNAs & Viral RNA degradation & $\begin{array}{c}\text { (Jaag and Nagy } \\
\text { 2009) }\end{array}$ \\
\hline $\begin{array}{c}5^{\prime}-3^{\prime} \\
\text { exoribonuclease } \\
\text { AtXrn4p }\end{array}$ & $\begin{array}{c}\text { Cucumber necrosis } \\
\text { virus (CNV) }\end{array}$ & Viral RNAs & $\begin{array}{l}\text { Emergence of novel viral } \\
\text { variants }\end{array}$ & (Cheng et al. 2007) \\
\hline $\begin{array}{l}\text { P0, varicose and } \\
\text { elF(iso) } 4 \mathrm{E}\end{array}$ & Potato virus A (PVA) & $\begin{array}{c}\text { Vpg and } \\
\text { RdRp HCpro }\end{array}$ & RNA translation & (Hafren et al. 2013) \\
\hline UBP1 & Potato virus $A$ (PVA) & $\begin{array}{c}\text { Vpg and } \\
\text { RdRp HCpro }\end{array}$ & RNA translation inhibition & (Hafren et al. 2015) \\
\hline $\mathrm{elF}($ iso) $4 \mathrm{E}$ & $\begin{array}{l}\text { Turnip mosaic virus } \\
\text { (TuMV) }\end{array}$ & VPg & $\begin{array}{l}\text { RNA translation and/or } \\
\text { replication }\end{array}$ & $\begin{array}{l}\text { (Beauchemin et al. } \\
\text { 2007) }\end{array}$ \\
\hline $\begin{array}{l}\text { exoribonuclease } \\
\text { Rrp6 /a small } \\
\text { alpha-heat } \\
\text { shock protein }\end{array}$ & Potyvirus & $\begin{array}{l}\text { Vpg and } \\
\text { HCpro }\end{array}$ & Infectivity & (Freire 2014) \\
\hline SGS3/RDR6 & $\begin{array}{c}\text { Potato virus } A \\
\text { (PVA) }\end{array}$ & Vpg & $\begin{array}{l}\text { Suppression of sense- } \\
\text { mediated RNA silencing }\end{array}$ & $\begin{array}{c}\text { (Rajamaki et al. } \\
\text { 2014) }\end{array}$ \\
\hline SGS3 & $\begin{array}{l}\text { Tomato yellow leaf } \\
\text { curl virus (TYLCV) }\end{array}$ & V2 & Supression of RNA silencing & (Glick et al. 2008) \\
\hline SGS3 & Rice stripe virus (RSV) & p2 & $\begin{array}{l}\text { Interfere with sense RNA } \\
\text { silencing }\end{array}$ & (Du et al. 2011) \\
\hline RDR6 & Rice yellow stunt virus & P6 & $\begin{array}{l}\text { Inhibition of secondary } \\
\text { siRNAs production and } \\
\text { systemic RNA silencing }\end{array}$ & (Guo et al. 2013) \\
\hline AGO1 & $\begin{array}{c}\text { Cucumber mosaic } \\
\text { virus (CMV) }\end{array}$ & $2 b$ & RNA silencing & $\begin{array}{l}\text { (Zhang et al. 2006; } \\
\text { Zhang et al. 2017) }\end{array}$ \\
\hline Nbrgs-CaM & $\begin{array}{l}\text { Tomato yellow leaf } \\
\text { curl betasatellite }\end{array}$ & $\beta C 1$ & $\begin{array}{l}\text { Suppression of RDR6 gene } \\
\text { expression and PTGS }\end{array}$ & (Li et al. 2014a) \\
\hline
\end{tabular}




\section{Plant virus interplay with cytoplasmic granules}

A study has shown that $A$. thaliana $60 \mathrm{~S}$ acidic ribosomal protein P0 copurified with Potato virus A (PVA) VPg and RdRp proteins. SGs and nuclear component elF(iso)4E colocalize with VPg-formed viral granules and PO as well. PO and elF(iso)4E both enable/enhance the infection process through protection of viral RNA from degradation. Overexpression of P0 promoted viral translation and required the viral $5^{\prime}$ UTR, while inhibition of viral cell-to-cell spread, competing for viral RNAs, also promoted translation by P0 and elF(iso)4E (Hafren et al. 2013). In another study, the helper component (HCPro) of PVA, amongst others known to act as suppressor of RNA silencing, also induces RNA granules that, besides P0 and elF(iso)4, contain the SG marker UBP1 and PB marker Varicose (VCS) and is down-regulated by VPg. Whereas HCPro and VCS stimulate VPg-promoted translation, UBP1 inhibits this process, indicating that PVA translation and potyviral RNA granules are interrelated (Hafren et al. 2015).

Turnip mosaic virus (TuMV), another potyvirus, has been observed to induce similar virus induced granules, and elF(iso)4E shown to interact with VPg which might play a key role in virus replication and translation (Hafren et al. 2015). Other translation associated factors like Eukaryotic elongation factor 1-alpha (eEF1A), Poly(A) binding protein 2 (PABP2) and chaperone heat shock cognate 70-3 protein have been observed in virus induced vesicles, and a subpopulation of eIF(iso)4E and PAPB2 to shuttle between cytoplasmic granules and nucleus during virus infection, and due to VPg-Pro (Beauchemin et al. 2007; Brengues et al. 2007). These studies indicate that TuMV might repress host nuclear mRNA translation and use the cytoplasmic translation machinery to accomplish viral RNA synthesis (Brengues et al. 2007). Interestingly, elF4E or elF(iso)4E is a nuclear-cytoplasmic shuttle protein involved in mRNA translation or storage (functioning in SG formation during stress) and in different plant species confers recessive resistance to plant viruses (Wang and Krishnaswamy 2012).

Recently, a $\mathrm{m}^{6} \mathrm{~A}$ demethylase ALKBH10B has been identified in Arabidopsis with a role in the RNA biology of viruses. The protein interacts with the coat protein of alfalfa mosaic virus (AMV) and removes $m 6 \mathrm{~A}$ in the AMV RNA genome. Upon silencing of this gene, systemic infection is largely inhibited. In vivo AtALKBH9B localizes to cytoplasmic bodies that perfectly colocalize with siRNA body component SGS3 and NMD component UPF1, and in $40 \%$ of the cases with $\mathrm{P}$ bodies component DCP1, suggesting that AtALKBH9B seems an intrinsic component of these RNA bodies and able to restrict AMV infection (Prasch and Sonnewald 2013). 
In yeast, the Lsm1p-7p/Pat1p/Dhh1p complex, which catalyses deadenylation of mRNA and promotes RNA degradation, has been shown pivotal for Brome mosaic virus (BMV) replication. BMV RdRp colocalizes with P bodies component Lsm1p and a deletion mutant of Ism7 has been shown to inhibit viral RNA translation (Anderson et al. 2015). Furthermore, viral RNAs have been found in P bodies and their 3' UTRs to be important for recruiting the decapping complex and switch BMV RNAs from translation into replication (Beckham et al. 2007). The 5'-3' exoribonuclease (Xrn) 4 is a component of $P$ body and acts downstream of DCP2, and functionally similar to yeast xrn1 (Souret et al. 2004). Expression of Xrn4 enhances viral RNA degradation during infection of plants with Cucumber necrosis tombusvirus (CNV), but also promotes the emergence of novel viral variants with different 5 ' truncations (Cheng et al. 2007). A similar inhibition is observed after Tomato bushy stunt tombusvirus (TBSV) infection (Beaudoin et al. 2009). Conversely, silencing of Xrn4 promotes systemic infection of $N$. benthamiana with TMV (Peng et al. 2011). ASYMMETRIC LEAVES 2(AS2), a recently identified $\mathrm{P}$ bodies component, interacts with the Cabbage leaf curl Virus (CaLCuV) nuclear shuttle protein BV1 and regulates plant mRNA decapping and degradation. BV1 expression induces AS2 transfer from the nucleus to cytoplasm to activate the decapping machinery. This activation accelerates the host plant mRNA metabolic rate, inhibits siRNA accumulation and thereby functions as an endogenous suppressor of PTGS, causing a susceptible condition for viral infection (Ye et al. 2015).

So far only very few cases have been reported that have demonstrated the interaction of viral proteins with SG components. In a recent study, the nuclear shuttling proteins (NSPs) from the (nuclear replicating) geminivirus Abutilon mosaic virus (AbMV) and nanovirus pea necrotic yellow dwarf virus (PNYDV) were identified as potential interactors of AtG3BP1 based on the presence of a sequence with similarity to the conserved G3BP-binding motif "FGDF" in the nsP3 from alphaviruses (Krapp et al., 2017). While both NSPs exhibited a nucleocytoplasmic distribution, upon stress induction both co-localized and interacted with atG3BP1, likely to suppress antiviral activity of SG or to support viral replication and dissemination. Due to the presence of "FGDF"-like motifs, Panas et al. (2015) earlier already suggested several potyvirus proteins to interact with G3BP as well.

Not only the $\mathrm{P}$ bodies degradation machinery and SG are targets for virus induced repression, also the siRNA and miRNA pathway are modulated by viruses. Aberrant RNAs resulting from RISC cleavage RNAs will enter $P$ bodies for further degradation. SiRNA bodies compete with PB for these aberrant RNAs, in which the two key elements suppressor of gene silencing 
3 (SGS3) and RDR 6 convert the aberrant RNA into dsRNA that will be processed into a population of secondary siRNAs and contribute to a stronger (antiviral) RNAi response. Potato virus $A(P V A)$ VPg interacts with SGS3 within siRNA bodies and represses the amplification by RDR6 (Beckham et al. 2007). This leads to the suppression of post transcription gene silencing and promotes accumulation of viral RNAs. Likewise, Plantago asiatica mosaic virus (PIAMV) TGB1, Tomato yellow leaf curl virus (TYLCV) V2 and Rice stripe virus (RSV) p2 interact with SGS3, while P6 protein of Rice yellow stunt virus (RYSV) interacts with RDR6, and inhibit the RDR6/SGS3--mediated amplification of secondary siRNAs (Du et al. 2011; Glick et al. 2008; Guo et al. 2013; Okano et al. 2014). By interfering with the RNAi amplification in siRNA Bodies many plant viruses suppress antiviral RNAi and are able to replicate to higher levels. CMV $2 b$ contains a GW motif that enables interaction with AGO1, thereby inhibiting the function of the PAZ and PIWI domains that are required for binding of the AGO within the RISC complex to (viral) target RNAs (Zhang et al., 2006 and 2017).

Plant viruses are also reported to interplay with NMD pathway in different ways. RNA viruses often contain internal stop codons to maximize coding potential, but these may also present targets for NMD. The genome of Turnip crinkle virus (TCV) contains two sequence domains that confer NMD-resistance. In this way, the virus is able to avoid subgenomic RNAs from being degraded and guarantee the expression of viral capsid protein (May et al. 2018). Potato virus X (PVX) with internal stop codons and long 3' UTRs is also prone to restriction by NMD (Garcia et al. 2014).

\section{Plant virus interplay with nuclear granules}

Considering the diverse function of nuclear granules and their role in DNA methylation and pre-mRNA synthesis and modification, it is also not a surprise that a number of viruses target these subnuclear RNA-protein condensation centres. Considering that two host proteins that reside in nuclear granules and are important in viral infection, namely coilin and fibrillarin, localize to both nucleolus and CBs (Trinkle-Mulcahy and Sleeman 2016), the following section will present a correct state of knowledge based on virus interaction and localization rather than type of granules.

Fibrillarin is a highly conserved rRNA methyltransferase involved in pre-rRNA processing, and accumulates in the nucleolus, but it is also an essential scaffold protein for CB. Fibrillarin is also essential for cell-to-cell movement, long-distance spread and systemic infection for 
several plant viruses, like Rice stripe virus or satellite virus of Bamboo mosaic virus (Chang et al. 2016; Zheng et al. 2015). Barley stripe mosaic virus (BSMV) and Umbravirus Groundnut rosette virus (GRV) hijack fibrillarin from the nucleus to form a functional RNP enabling them for cell-to-cell movement (Canetta et al. 2008; Kim et al. 2007a; Li et al. 2018b). For several other viruses, namely Poa semi-latent virus (PSLV), Grapevine red blotch-associated virus (GRBaV) and Potato virus A (PVA), interactions with fibrillarin have been identified, but the reason for this is still not clear (Gu et al. 2015; Rajamaki et al. 2014; Semashko et al. 2012) (Guo et al. 2015; Rajamaki et al. 2014; Semashko et al. 2012). The 2b protein of Cucumber mosaic virus (CMV) also localizes in the nucleolus (Du et al. 2014). Interestingly, the $2 \mathrm{~b}$ protein shuffles between the nucleus, nucleolus, and cytoplasm; the nucleolar pool is necessary for CMV virulence and symptom development, while the cytoplasmic pool of $2 b$ controls the suppression of RNA silencing (Du et al. 2014). In the cytoplasm, $2 b$ interacts with AGO1, while interactions with AGO4 occur predominantly in the nucleus, but not nucleolus (Gonzalez et al. 2010). Furthermore, 2b binding to AGO4 is necessary for the suppression of RDR1/6 - dependent antiviral silencing (Fang et al. 2016). Lastly, also p37 of Tombusvirus Pelargonium line pattern virus (PLPV) resides in the nucleolus together with fibrillarin (Perez-Canamas and Hernandez 2018). However, the nucleolar localization is not fibrillarin-dependent.

Proteins of Importin alpha family are important in cytoplasm-nucleus trafficking (Miyamoto et al. 2016). Recently, it has been shown that two plant viruses interact with them. P37 of Tombusvirus Pelargonium line pattern virus (PLPV) interacts with distinct members of the importin alpha family in the nucleolus and other uncharacterized subnuclear foci, and these interactions promote PLPV infectivity (Perez-Canamas and Hernandez 2018). Potato moptop virus (PMTV) TGB1 protein is a part of the cell-to-cell movement protein complex. TGB1 interactions with importin alpha in the nucleus and nucleolus are essential for viral systemic movement (Lukhovitskaya et al. 2015).

Coilin is a nuclear phosphoprotein that is an indispensable structural element of CBs. Although the protein was cloned almost 30 years ago, its function remains elusive (Machyna et al. 2015). In CBs, it plays a role in snRNP biogenesis (Stanek 2017), while the nuclear pool of coilin suppresses pol I transcription during certain stress conditions (Gilder et al. 2011). As CBs and nucleolus are functionally related, they are expected to have a role during viral infections. Indeed, plants with down-regulated expression of the coilin gene showed altered responses to virus infections (Shaw et al. 2014). For some viruses, like BSMV, Tobacco rattle 
virus (TRV), tomato black ring virus (TBRV) and tomato golden mosaic virus (TGMV), the deficiency of coilin seems to promote viral replication, while replication of Potato virus Y (PVY) and Turnip vein clearing virus (TVCV) seems to be hindered (Shaw et al. 2014). However, the exact function of coilin in viral infection remains obscure.

ALY proteins are recently described to have a role in RNA export from the nucleus to the cytoplasm. In Arabidopsis, four ALY proteins have been identified so far (Pfaff et al. 2018). ALY1 and ALY2 proteins often are localized to speckles and foci within the nucleoplasm, while ALY3 and ALY4 were also found in the nucleolus (Pfaff et al. 2018; Wang et al. 2017a). Interestingly, evidence is accumulating that they are important players during viral infections. During Tomato yellow leaf curl virus (TYLCV) infection, ALY4 translocates from the nucleolus to further uncharacterized nuclear speckles (Wang et al. 2017a). Importantly, transiently expressed CP of TYLCV localizes with ALY4 in the nucleolus but no interactions between ALY and viral proteins have been described (Wang et al., 2017a). P19 protein of Tomato Bushy Stunt Virus (TBSV) is a RNA silencing suppressor, but its presence is also required for short- and long-distance virus movement and symptom development. P19 interacts with Arabidopsis ALY2-4 proteins in the nucleus and with ALY1 in the nucleolus (Canto et al. 2006). The interactions lead to translocation of ALY2 and ALY4 from the nucleus to the cytoplasm (Uhrig et al. 2004). While ALY1 does not relocate in the presence of P19, it hinders P19 in suppressing RNA silencing (Canto et al. 2006). Interestingly, mutant plants lacking ALY1 are hindered in RdDM caused by mRNA trafficking of AGO6 and the activities of RNA Polymerase V (Choudury et al. 2019), both crucial for silencing of geminiviruses during the recovery phase of infection (Coursey et al. 2018).

\section{Conclusions and outlook}

While the role of cytoplasmic and nuclear RNA granules in the regulation of cellular processes, homeostasis and (a)biotic stress responses is clearly recognized, ongoing studies keep unveiling more details that indicate their complexity, interplay and involvement in more processes than initially thought of. Studies on viruses simultaneously turn out to present helpful tools in this. While in the past decade this field of science has more progressed with animal infecting viruses, due to the conservation of many RNA regulation processes in eukaryotes, knowledge obtained with animal infecting viruses can be taken as interesting leads for studies with plant viruses. Plant cells and animal cells, however, do exhibit differences in their cellular architecture and organization as well exemplified by e.g. 
1) the presence of siRNA bodies, for the amplification of RNAi, in plant cells and their absence from animal cells, 2) the absence of GW-182 bodies from plant cells and their presence in animal cells, 3 ) seeming differences in the cellular localization of si/miRNA-mediated RNA silencing in plant versus animal cells. On the other hand, with ongoing studies revealing the existence of P bodies-like bodies (GW-182), SG-like bodies and CB-like bodies, indicating a growing complexity of RNA granules and their transiently different status, the regulation of various processes in plants and animal cells in the end might turn out to be not so much different at all. This idea is supported with the observation that plant proteins have been identified with functional/structural similarities to certain animal proteins e.g., plant host proteins containing GW motifs and exhibiting a functional similarity to the animal GW-182 protein, or the plant COP1 protein with functional/structural similarity to the nuclear PML protein of animal cells (Reyes 2001).

While we are just at the start of an era where studies on plant viruses and the interplay with RNA granules receive growing attention, studies that are reported on this often have remained elusive as to the how and why of the interplay in relation to the benefit for viral replication and dissemination and/or counter defence strategies against antiviral responses. On the other hand, details on the molecular biology and genetics of viruses may also point to issues relevant for the mechanism of RNA regulation and dynamics related to RNA granules. Viruses that could be of special interest and support to these studies are families containing plant- and animal infecting viruses, e.g. rhabdoviruses and bunyaviruses. Due to structural and functional homologies between the genomes and proteins of the plant- and animal-infecting counterparts, but having to cope with the host cell machinery that seems to differ in architecture and organization (plant vs animal), turns the research on the interplay between those viruses and RNA granules into a very interesting challenge.

Lets look at a few examples. In the past few years, studies in animal cells have revealed a cellular mechanism that acts antiviral and represses CpG/UpA dinucleotides in animal RNA viruses (Fros et al. 2017). This repression does not involve a changed phosphorylation status of elF2 $\alpha$ (SG inducer). Instead, a zinc-finger antiviral protein (ZAP) has been shown to be involved in the selective binding to $\mathrm{CpG}$ sequences and viruses high in $\mathrm{CpG}$ contents are only able to replicate to wild type virus levels in ZAP knock out cells (Takata et al. 2017). A recent study showed that ZAP can be found in the cytoplasm but transiently localizes to SG during (Sindbis) virus replication. The antiviral activity correlated with the ability to localize in SG 
and support the idea of SG presenting an important hub in antiviral defence (Law et al. 2019). Furthermore, in another study the enterovirus $A 713 C$ protease has been shown to cleave ZAP (Xie 2018), clearly as a means to (partly) escape from this (antiviral) host response. Considering that plant genomes also exhibit CpG repression, it will be interesting to find out whether in plants a similar mechanism exists that represses $\mathrm{CpG}(/ \mathrm{UpA})$ dinucleotides and also acts on plant viral RNA genomes.

Another interesting question relates to viruses that rely/benefit on $\mathrm{P}$ bodies for genome transcription/replication, e.g. bromoviruses and bunyaviruses. Localizing at $\mathrm{P}$ bodies makes their genomic (m)RNA vulnerable and prone to degradation by the decay machinery. However, bromoviruses do not contain a poly(A)-tail, but a 3'UTR that folds in a tRNA-like structure and is not prone to deadenylation. Maybe it is for this reason that bromoviral RNAs, localizing at $P$ bodies (Beckham et al. 2007), resist against degradation and are able to replicate. A similar question pops up with bunyaviruses where several studies on SNV hantavirus have shown a link to $P$ bodies as sites of viral genome transcription (Mir et al. 2008a). Viral mRNAs of hantaviruses, as with all bunyaviruses, do not contain a common eukaryotic poly(A)-tail. If hantaviruses/bunyaviruses localize at $P$ bodies from where these viruses would collect host capped-RNA leader sequences for genome transcription initiation, it would seem logic to assume that these viruses have evolved strategies to protect or take their non-polyadenaylated viral mRNAs away from $\mathrm{P}$ bodies to prevent degradation by the RNA decay machinery. Whether this involves a similar (sequence-dependent) strategy as observed with TCV (May et al. 2018) where the viral genome contains NMD-resistant sequences to prevent degradation of viral RNA at P bodies, remains to be investigated. In light of this, it is also interesting to note that a recent study has shown that the polypyrimidine tract binding protein 1 (PTBP1) protects specific retroviral and host cellular mRNAs with long 3'UTR sequences from the NMD pathway. Binding of PTBP1 in the 3'UTRs on pyrimidine-rich stretches near termination codons (TCS) prevents binding of UPF1, a protein that once bound disposes (even functional) transcripts to the NMD pathway (Ge et al. 2016). Bunyaviral mRNAs do not contain a common eukaryotic poly(A)-tail, but some members instead contain a long 3'UTR that is predicted to fold into a stable hairpin structure (Kormelink, 2011). Furthermore, some bunyavirus mRNAs contain just downstream the TC stretches rich in C- and U- residues. Intriguingly, tomato spotted wilt virus (TSWV) mRNAs also contain sequence signatures within their 3'UTR that resemble ARE (AUUUA) elements, which are known to destabilize mRNAs (Geerts-Dimitriadou et al. 2012). Whether these are biologically functional and via RNA binding proteins target TSWV mRNAs to P bodies for 
degradation remains to be investigated. In retrospect, and in light of the intimacy between $P$ bodies and SG, in which many RNA molecules and proteins are exchanged, one might also question whether or not hantaviruses/bunyaviruses use SG as source for capped RNA leaders as well (Mir et al., 2008), but also U bodies could present a source. U bodies have been discovered not long ago and present RNP structures that contain Uridine-rich, capped small nuclear (sn)RNAs that mature in the cytoplasm and also associate with P bodies (Liu and Gall 2007), but finally localize in the nucleus where they play a key role in (RNA Pol II complex- mediated) pre-mRNA processing. Support for $U$ bodies as being another source comes from two recent studies on Influenza virus transcription where snRNAs presented a preferred source for capped-RNA leaders (Gu et al. 2015; Koppstein et al. 2015).

While at this moment many questions on the cellular RNA communication network remain, finding the answer to these will not only advance our understanding and the importance of RNA sequence elements and host proteins in this, but also help to understand how viruses have evolved to modulate and benefit from the RNA communication network and simultaneously repress and/or evade network activities that would otherwise inhibit viral replication and dissemination. In relation to this, a recent system-wide profiling of the RNAbinding proteins network has unveiled unknown key regulators of virus infection (GarciaMoreno et al. 2019). In a study on SINV infection of animal cells more than 200 RNA binding proteins (RBPs) were observed to alter their binding profile, due to the loss of host mRNA and accumulation of viral RNA. RBPs were observed to redistribute to viral factories, likely to enable viral infection but also as a means of the host to defend against viral invasion. While the exonuclease XRN1 is generally assumed to present an antiviral factor, this protein appeared essential for SINV replication. Gemin5, a protein of the SMN complex, required for snRNP assembly, appeared to co-localize with SINV RNA in viral factories and by binding to the 5'-Cap inhibited translation by interference with ribosomes (Garcia-Moreno et al. 2019).

The interplay of viruses with the cellular RNA communication network is of evident importance if viruses want to benefit as much as possible but also prevent from becoming sensed by RNA surveillance and control mechanisms of the host, aimed at homeostasis and to cope with (a)biotic stress responses. Not perse and necessarily directed at viral clearance, but at RNA control and regulation, to which viral (m)RNA will be subjected as well. 



\section{Chapter 3}

\section{Dynamic transcriptional profiles of Arabidopsis thaliana infected by Tomato spotted wilt virus}

Min Xu, Jing Chen, Ying Huang, Danyu Shen, Yi Xu and Xiaorong Tao
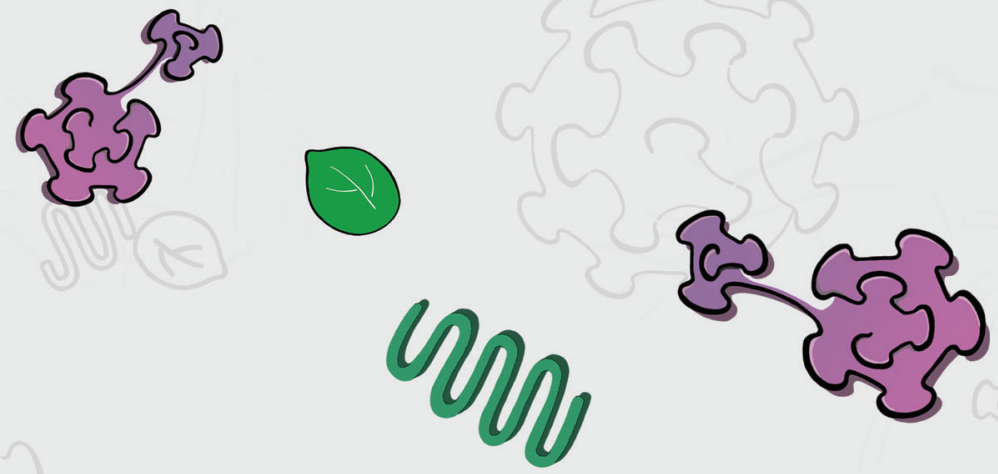

This chapter has been published in a slightly modified version as:

Xu, M., Chen, J., Huang, Y., Shen, D., Sun, P., Xu, Y., and Tao, X. 2020. Dynamic transcriptional profiles of Arabidopsis thaliana infected by Tomato spotted wilt virus. Phytopathology 110:153-163. 


\section{Abstract}

Tomato spotted wilt virus (TSWV) is a negative-stranded RNA virus that infects hundreds of plant species, causing great economic loss. Infected Arabidopsis thaliana develop symptoms including chlorosis and wilt, which can lead to cell death. From 9 days to 15 days after TSWV infection, symptoms progress through a three-stage process of appearance, severity, and death. In the present study, deep sequencing technology was firstly used to explore gene expression in response to TSWV infection in model plant $A$. thaliana at different symptom development stages. We found that plant immune defence and protein degradation are induced by TSWV infection, and that both inductions became stronger over time. Photosynthesis pathway is attenuated with TSWV infection. Cell wall metabolism has a large extent of downregulation while some genes are upregulated. These results illustrate the dynamic nature of TSWV infection in A. thaliana at the whole-transcriptome level. The link between biological processes and sub-pathway metabolism was further analysed. Our study provides new insight into host regulatory networks and dynamic processes in response to TSWV infection. 


\section{Introduction}

Tomato spotted wilt virus (TSWV) is a negative-stranded RNA virus of the genus Orthotospovirus, the family Tospoviridae, and the order Bunyavirales. TSWV is widely distributed throughout the world, and can cause huge economic losses by infecting a range (>800) of plant species, including tomato, pepper, lettuce, peanut, and chrysanthemum (Oliver and Whitfield 2016; Scholthof et al. 2011; Turina et al. 2016). In nature, TSWV is transmitted mainly by western flower thrips (Frankliniella occidentalis) in a persistent and propagative manner (Gilbertson et al. 2015; Rotenberg et al. 2015). Western flower thrips are difficult to control because they are small and often concealed in buds, where they feed on plant pollen and organs. TSWV is considered the second-most scientifically or economically important plant virus (Scholthof et al. 2011). Plant stems, leaves, and fruits develop severe symptoms after viral infection. In the early stages, leaves and fruits display chlorotic rings, mottling, and flecking, which progress to stunting necrosis. Finally, the whole plant becomes wilted and dies (Prins and Goldbach 1998). In China, TSWV is distributed mainly in the southwestern area, and the virus causes serious harm within the tomato industry, leading to losses of up to nearly $100 \%$ (Hu et al. 2011).

The TSWV genome is composed of three RNA segments, which are 9 kilobases $(\mathrm{kb}), 4.8 \mathrm{~kb}$, and $3 \mathrm{~kb}$ in size. The large (L) segment encodes an RNA-dependent RNA polymerase. The medium (M) segment encodes the NSm protein and the precursor of glycoprotein. NSm is a movement protein that localizes at the endoplasmic reticulum (ER) and plasmodesmata to facilitate viral cell-to-cell movement (Feng et al. 2016). The small (S) segment encodes the NSs nonstructural protein and the N protein. NSs is a gene-silencing suppressor that can bind miRNA and siRNA duplexes, as well as long dsRNAs that inhibit the cleavage of viral dsRNAs from Dicer protein (Hedil et al. 2017; Hedil et al. 2015). N protein is a nucleocapsid protein that is able to move along actin with the help of $\mathrm{XI}-\mathrm{K}$, and can bind and protect viral RNA in vivo (Feng et al. 2013; Li et al. 2015). The L segment is composed of negative-sense RNA, while the other two segments are composed of ambisense RNA.

Plant viruses are obligate parasites that require host machinery to accomplish multiplication. Plant metabolic pathways change dynamically during viral infection (De Vos et al. 2005; Mochizuki et al. 2014; Prasch and Sonnewald 2013). In turn, host plants have evolved various means of preventing the spread of viruses (Petek et al. 2014; Shi et al. 2013; Su et al. 2016; Zhu et al. 2019). Previous studies have shown that viruses can induce metabolic 
changes during interactions with host plants. For example, in pea plants, the expression patterns of genes involved in chloroplastic metabolism change to release reactive oxygen species (ROS) in response to plum pox virus infection (Diaz-Vivancos et al. 2008; Rubio et al. 2015). Differential expression patterns are observed in both resistant and susceptible tomato cultivars infected with tomato yellow leaf curl virus (TYLCV) (Sade et al. 2014). TSWV triggers plant ROS accumulation in hypersensitive and susceptible Solanaceae hosts (Quecini et al. 2007). In insect host whitefly, global transcriptional response after TYLCV infection was also analysed and pathways related to virus-host interaction were identified (Luan et al. 2011). Also taking TYLCV for an example, it has been shown that in whiteflies, autophagy pathway participates in resistance to its infection (Wang et al. 2016). From viral aspect, BV1 protein could subvert plant resistance through interacting with transcriptional factor MYC2 (Li et al. 2014b).

The development of RNA-sequencing (RNA-seq) techniques has provided new insights into host gene transcriptional changes during biotic and/or abiotic stress (Wang and Liu 2009). This technology has been widely used to study changes in gene expression in virus-host interactions (Geng et al. 2017; Ke et al. 2014; Miozzi et al. 2014; Yates et al. 2014; Yu et al. 2012). For example, analyses of gene expression changes in Plum pox virus-infected peach leaves have revealed the complicated expression process for Sharka symptoms (Rubio et al. 2015). Comparative gene expression analyses have shown that primary metabolism and ubiquitin-proteasome pathways in Sogatella furcifera are perturbed by infection with southern rice black-streaked dwarf virus (Xu et al. 2012). For tospoviruses, microarray assay has been performed on Nicotiana benthamiana plants infected with sonchus yellow net virus (SYNV) and impatiens necrotic spot virus (INSV) (Schneweis et al. 2017). Likewise, microarray assay on analysis of specific and common gene expression changes in hosts infected with TSWV and other RNA viruses have been revealed in Dendranthema grandiflorum Ramatuelle cultivar Shinma (Choi et al. 2015), as well as in tomato shoots and roots (Catoni et al. 2009). To study transcriptional response in insect host after TSWV infection, RNA-seq has been done to analyse transcriptome profile at different host developmental stages (Senthil et al. 2005). However, no deep sequencing on type species TSWV and plant host interaction has been performed.

TSWV can cause rapid death when it infects host plants, which makes it an attractive target for studies on host gene expression variation and metabolic changes from initial infection to plant death. TSWV is able to infect the model plant Arabidopsis thaliana. 
At-4/1 was identified from $A$. thaliana as an interactor with movement protein to facilitate viral movement (Paape et al. 2006). Candidate proteins were identified from A. thaliana to help in replication of TSWV (Kainz and Hoopes 2009). Arabidopsis is one of the most studied hosts plants used to explore mechanisms of interaction with pathogens, and it plays an important role in research on pathogenicity and the plant immune response (Asari et al. 2017; Huang et al. 2016; Martinez-Perez et al. 2017; Raad et al. 2019; Zhang et al. 2015).

In the present study, deep sequencing was performed to analyse the differentially expressed genes at $9 \mathrm{dpi}, 12 \mathrm{dpi}, 15 \mathrm{dpi}$ in model plant $A$. thaliana after TSWV infection. In addition, viral transcripts from these data were collected to be analysed on their host cellular-derived RNA leader sequences. Key host genes were comprehensively identified and classified into essential pathways, providing new insight into TSWV pathogenesis and the host immune response. Our research illustrates the symptom development process in light of global transcriptome reprogramming after TSWV infection.

\section{Materials and Methods}

\section{Plant material and virus inoculation}

A. thaliana was grown in growth chambers (Jiangnan Motor Factory, Ningbo, China) at $22^{\circ} \mathrm{C}$ with a $8 \mathrm{~h}$ light/16 $\mathrm{h}$ dark cycle. TSWV was maintained in $N$. benthamiana plants. Six- to eight-week-old plants were used for viral inoculation. $N$. benthamiana leaves with TSWV were ground in $\mathrm{PB}\left(0.01 \mathrm{M} \mathrm{Na} \mathrm{HPO}_{4} \cdot \mathrm{H}_{2} \mathrm{O}, 0.01 \mathrm{M} \mathrm{Na} \mathrm{H}_{2} \mathrm{PO}_{4} \cdot 2 \mathrm{H}_{2} \mathrm{O}, \mathrm{pH} 7.5\right)$ buffer to inoculate A. thaliana. The negative control was inoculated with $\mathrm{PB}$ buffer.

\section{RNA Isolation and RNA-seq Library Preparation}

Total RNA of systemically infected $A$. thaliana leaves was extracted using a Total RNA Extraction Kit (Tiangen, Beijing, China). For each sample, total RNA was used to prepare the mRNA-seq library according to the TrueSeq RNA Sample Prep Kit protocol (Illumina, USA). Library quality control and quantification were performed using the Experion ${ }^{\mathrm{TM}}$ DNA $1 \mathrm{~K}$ Chip (Bio-Rad, USA) and Qubit fluorometer (Invitrogen, USA), respectively. For each library, 75 million 48 bp paired-end sequences were generated using an Illumina HiSeq 2500 sequencer. 


\section{Quantitative real-time PCR (qRT-PCR)}

Total RNA from systemically infected $A$. thaliana was used to synthesize first-strand cDNA using a PrimeScrip RT reagent kit with gDNA eraser (Takara, Dalian, China). Then the cDNA was amplified using Power SYBR Green Master Mix (Life Technologies, USA). Primers used in qRT-PCR for validation of differentially expressed genes are listed in Table 3.2. The qRT-PCR was performed using an ABI 7500 Real-Time PCR system (Life Technologies). Actin served as an internal control to normalize the RNA levels of target genes between samples.

\section{Reads mapping and annotations}

A total of 18 RNA libraries were sequenced in one HiSeq 2500 channel, and adapter trimming and cleaning of the reads was carried out. The produced clean reads were mapped to the Arabidopsis (2012) reference genome (http://mapman.gabipd.org/) using TopHat software with default parameters (Trapnell et al. 2009). Only the unique mapped reads were retained for subsequent processing. The retained reads were quantified using the Cufflinks v1.0.3 program (Trapnell et al. 2010), and the expression level of each gene was calculated by normalizing to FPKM values. To filter out weakly expressed genes, only genes with FPKM values $>1$ were included in the analyses. Groups of three biological replicates were combined and differentially expressed genes were identified using the GFOLD algorithm (Feng et al. 2012). Genes with two-fold or greater change changes in expression compared to the controls (FDR < 0.05) were considered differentially expressed (Klipper-Aurbach et al. 1995). The absolute value of $\log 2$ ratio $\geq 1$ was used as the threshold for significant differences in gene expression.

\section{Software and websites used for analyses}

The MapMan ontology tool was used to obtain an overview of $A$. thaliana genes involved in metabolic pathways. MapMan uses a plant-specific ontology that classifies genes into well-defined hierarchical categories, designated BINs. A. thaliana genes were assigned to BINs using the Mercator automated annotation pipeline. MeV software was used for nonsupervised clustering analyses of selected expression data with Euclidean-related metrics (Manhattan distance) and a complete clustering algorithm (TIGR MeV software package) (Saeed et al. 2003). The Venn diagrams (http://bioinformatics.psb.ugent.be/webtools/ Venn) website was used to create GO annotation images and Venn diagrams. 


\section{Results}

\section{Symptom development in TSWV-infected A. thaliana}

A. thaliana plants were mechanically inoculated with TSWV, and the symptom development process was observed (Fig. 3.1). At 9 dpi, small yellow necrotic spots appeared on inoculated leaves, and no symptoms were observed on top leaves (Fig. 3.1A, upper panel). By $12 \mathrm{dpi}$, yellow necrotic spots had spread over entire leaves, while top leaves began to display a light-yellow appearance (Fig. 3.1A, lower panel). At $15 \mathrm{dpi}$, inoculated leaves were dying, and the systemically infected leaves developed curl and yellowing wilt symptoms, indicating that the entire plant was undergoing systematic death (Fig. 3.1A). At these three stages, all TSWV-infected plants exhibited stunt to some extent compared to mock-inoculated plants (Fig. 3.1). Viral N mRNA expression was checked by qRT-PCR (Fig. 3.1B). To compare changes in metabolic regulation between different stages after virus infection, three biological replicates of each stage were prepared for genome-wide sequencing using RNA-seq. For each biological replicate, we collected systemically infected leaves of three different plants for RNA extraction and sent out for high throughput sequencing.

A
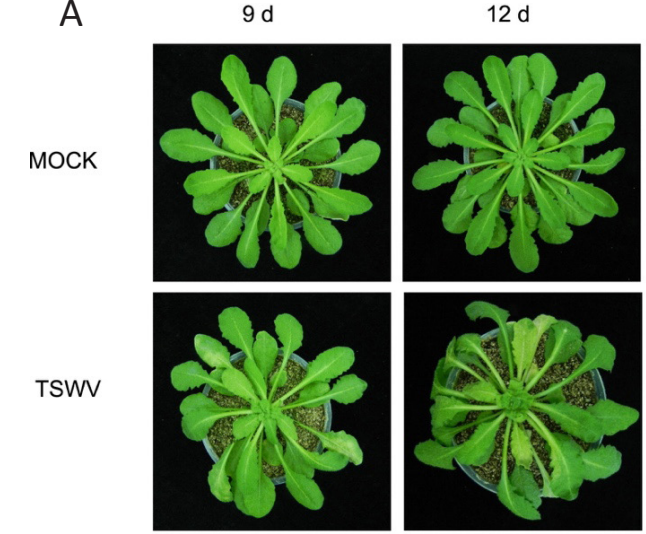

B

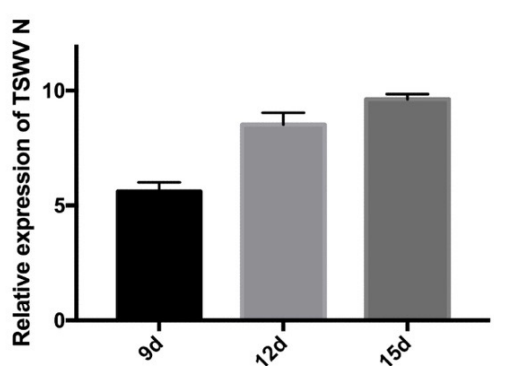

$15 d$
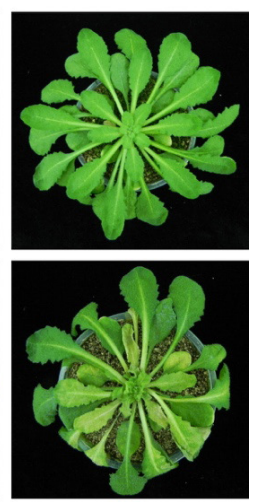

Figure 3.1 Symptoms of TSWVinfected $A$. thaliana plants. (A) Upper panel represents symptoms in mock-inoculated plants 9 days, 12 days, 15 days post-inoculation (dpi), respectively. Lower panel represent symptoms in TSWVinoculated plants at 9 days, 12 days, and $15 \mathrm{dpi}$, respectively. (B) qRT-PCR of TSWV N mRNA expression level. 


\section{Overview of expression patterns of differentially expressed genes in infected plants at different stages}

A total of 18 RNA libraries were sequenced, and within each library $>86 \%$ of reads were mapped to the $A$. thaliana reference (Supplemental Table S3.1). We identified 33,279 transcripts in total, and obtained three libraries of 8,916 deferentially expressed genes (DEGs) (Supplemental Table S3.2). These included 5,004, 5,798, and 6,913 DEGs at 9 dpi, 12 dpi, and $15 \mathrm{dpi}$, respectively (Supplemental Table S3.3).

We identified 3,090 genes with consistent expression across the three different stages (Fig. 3.2). Among them, 1,624 genes were upregulated and 1,448 genes were downregulated at all three timepoints. Some genes were differentially expressed at only a single timepoint: 482, 350, and 935 genes were upregulated only at $9 \mathrm{dpi}, 12 \mathrm{dpi}$, and $15 \mathrm{dpi}$, respectively, while 271,522 , and 898 genes were downregulated only at $9 \mathrm{dpi}, 12 \mathrm{dpi}$, and $15 \mathrm{dpi}$, respectively.

To identify other gene expression patterns, 5,103 genes were analysed using MEV software to reveal the expression patterns of individual genes (Supplemental Table S3.4). Genes with similar expression patterns were clustered together, resulting in four groups of gene expression patterns (Fig. 3.3).
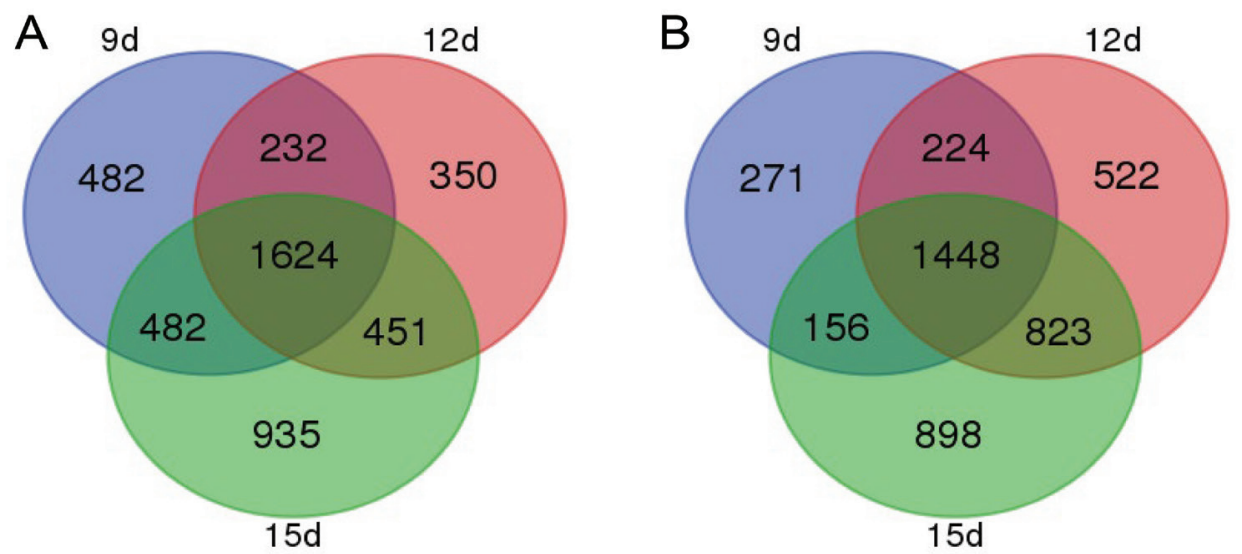

Figure 3.2 Venn diagrams of shared and unique differentially expressed transcripts at the three developmental stages. (A) depicts upregulated transcripts and (B) depicts downregulated transcripts. Blue represents transcripts at $9 \mathrm{dpi}$, red represents transcripts at $12 \mathrm{dpi}$, and green represent transcripts at $15 \mathrm{dpi}$. 


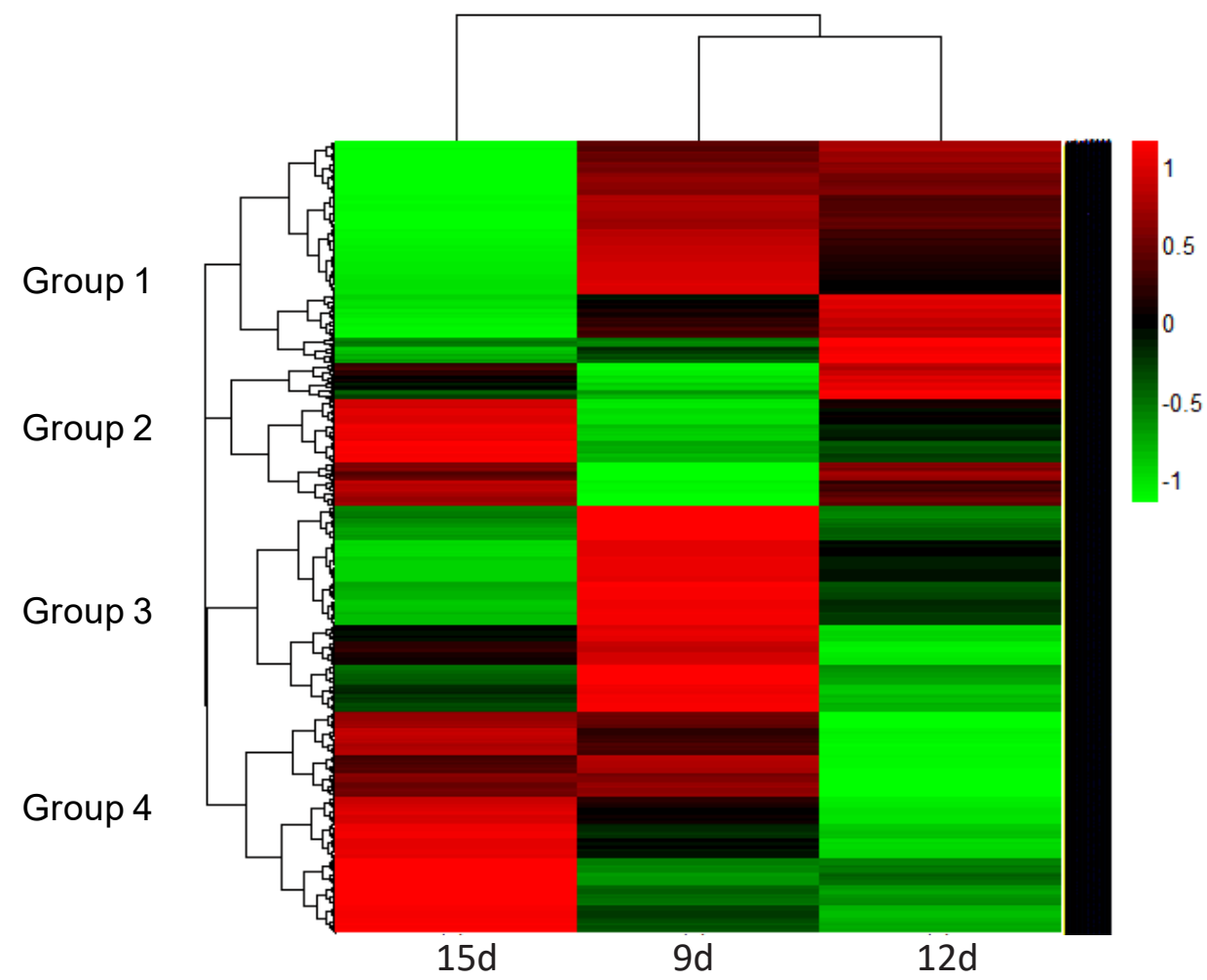

Figure 3.3 Hierarchical clustering of 5102 DEGs at different timepoints. Log2 (fold change) values $>1$ were used. Fold changes from -1 to 1 are shown in a gradation from green to red. The left lane represents DEGs at $15 \mathrm{dpi}$, the middle lane represents DEGs at $9 \mathrm{dpi}$, and the right lane represents DEGs at $12 \mathrm{dpi}$.

\section{Gene ontology enrichment and metabolic pathway analyses of transcriptional responses to TSWV infection}

To elucidate physiological changes at the three different timepoints on a macroscale, the agriGO analysis tool was used for parametric analysis of gene set enrichment (PAGE) for upregulated and downregulated genes. Gene Ontology (GO) enrichment classification revealed that the DEGs were associated with a range of biological processes, molecular functions, and cellular component categories. PAGE analyses were used to compare several rows of DEGs simultaneously (Fig. 3.4). At 9 dpi, cell killing, death, multi-organism process, response to stimulus, and immune system process were the top five most strongly induced pathways. Cellular component organization, cell wall organization or biogenesis, macromolecular process, organelle part, and structural molecule activity were the top five 
most strongly inhibited pathways. Compared to the $9 \mathrm{dpi}$ timepoint, pathways at $12 \mathrm{dpi}$ were moderately regulated. Cell killing, death, and immune system process were also induced. Organelle and extracellular region part were relatively inhibited. At $15 \mathrm{dpi}$, signalling process and transcription regulator activities were induced while cell wall organization, extracellular region, organelle part, and catalytic activity were inhibited (Fig. 3.4).

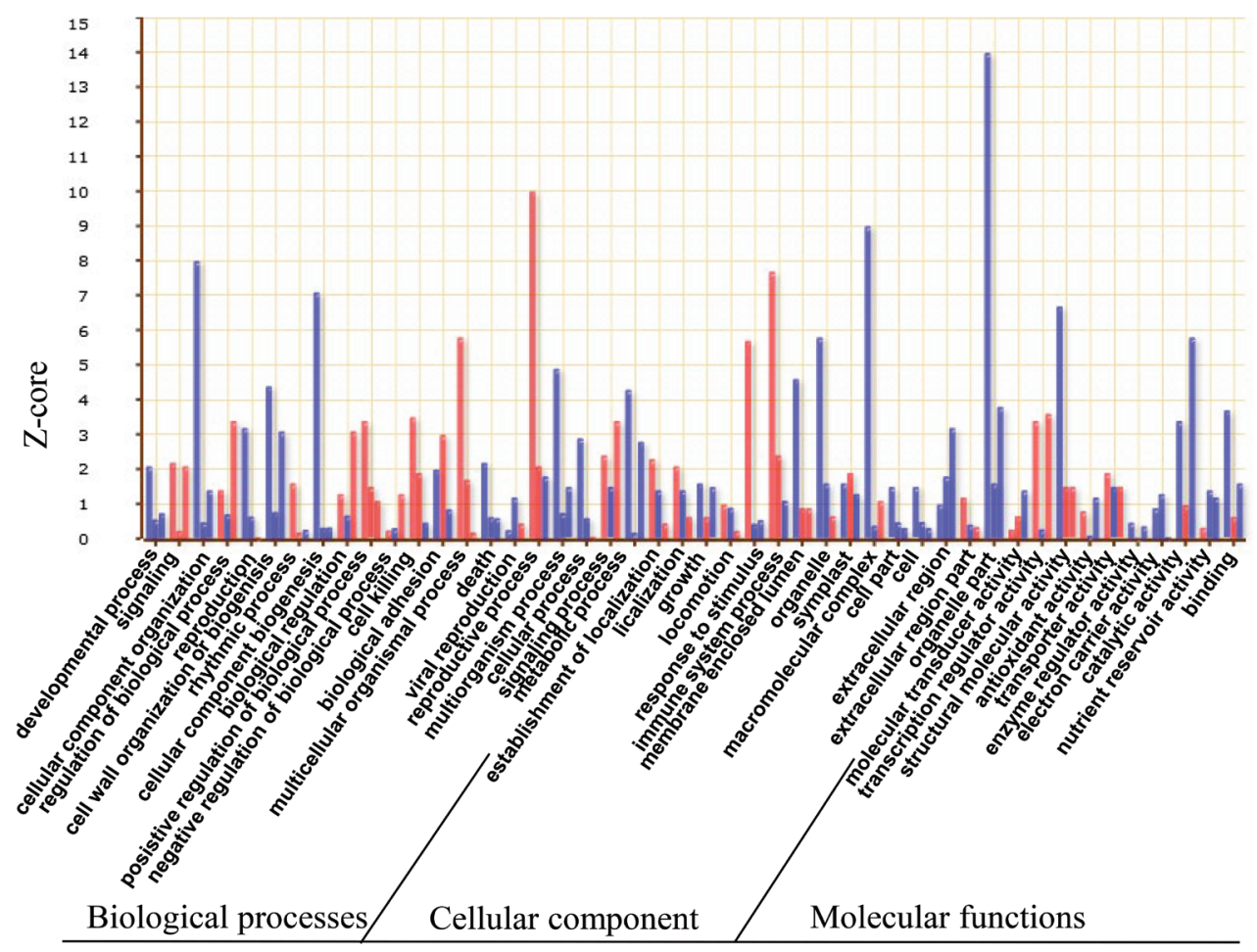

Figure 3.4 GO annotation results for DEGs. Forty-six sub-pathways are listed on the $\mathrm{X}$ axis below the bar chart. The three bars on each sub-pathway represent DEGs at $9 \mathrm{dpi}, 12 \mathrm{dpi}$ and $15 \mathrm{dpi}$. Z-core represents DEG values calculated in each group. Red indicates upregulation and blue indicates downregulation.

Then the MapMan tool was used to present individual gene responses and their related metabolic pathways. Hotspot diagrams of each gene at $9 \mathrm{dpi}, 12 \mathrm{dpi}$, and $15 \mathrm{dpi}$ are shown in Supplemental Table S3.2. The photosynthesis pathway tended to be incrementally repressed, consistent with the phenotype of chlorosis and wilt on leaves (Supplemental Fig. S3.1). Cell wall modification and amino acid synthesis were also repressed. By contrast, genes related to lipid metabolism exhibited induced expression. In the secondary metabolism pathway, terpene synthase genes were incrementally repressed while phenylpropanoid pathway 
genes were incrementally induced (Supplemental Fig. S3.1). These results represent an overview of changes to cellular metabolic pathways in response to TSWV infection at different points in time.

\section{Photosynthesis is inhibited by TSWV infection}

As shown in Supplemental Fig. S3.1, metabolic pathway analyses showed that photosynthesis was downregulated by TSWV infection. Non-supervised clustering analyses were performed to obtain a more precise overview (Fig. 3.5). The expression of genes in the photosynthesis pathway were mostly downregulated, and all downregulated genes exhibited similar expression patterns regardless of the amplitude of the variation. We noticed an increasing

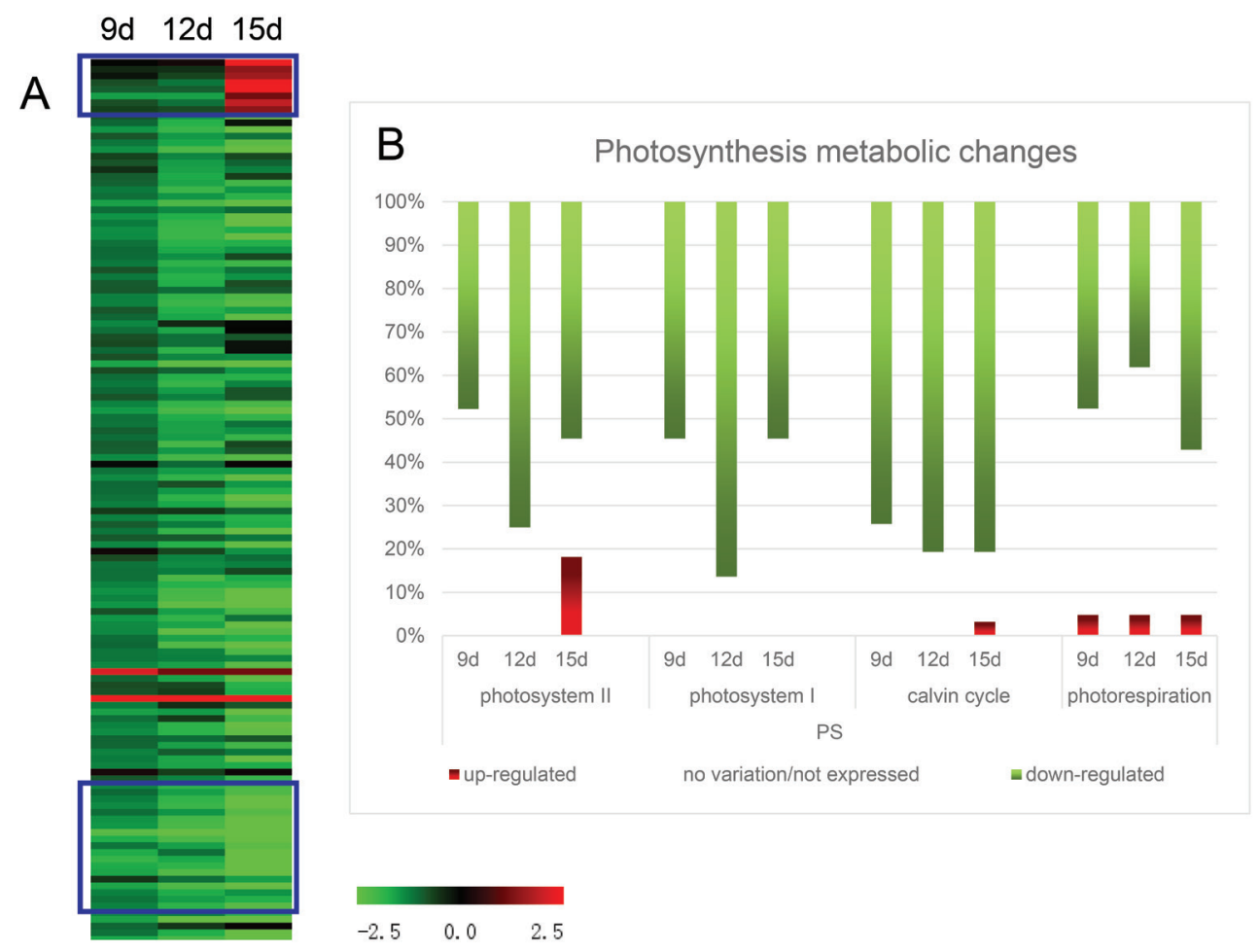

Figure 3.5 Analysis of photosynthesis-related pathways. (A) Gene expression heatmap at 9 dpi, 12 dpi, and 15 dpi. Top blue box represents the LHC-II system, which was upregulated at $15 \mathrm{dpi}$, and bottom blue box represents the Calvin cycle, which was downregulated at $12 \mathrm{dpi}$ and $15 \mathrm{dpi}$. Log2 value is 2.5. (B) Percentage of DEGs in photosynthesis metabolic sub-pathways at $9 \mathrm{dpi}, 12 \mathrm{dpi}$, and $15 \mathrm{dpi}$. Red bars represent upregulated genes and green bars represent downregulated genes. 
fold change over time after viral infection (Fig. 3.5A). At $9 \mathrm{dpi}$, the photosynthesis pathway exhibited mild downregulation, which became stronger at $12 \mathrm{dpi}$. At $15 \mathrm{dpi}$, the entire photosynthesis pathway was nearly completely shut down, as indicated by very low expression levels (Fig. 3.5). For instance, ribulose bisphosphate carboxylase small chain $1 \mathrm{~A}$ (At1g67090), which is a rubisco small subunit that plays a role in copper ion binding, was downregulated from FPKM value 23,581.5 to 7,752.3 at $9 \mathrm{dpi}$, from 20,853.4 to 4,349.6 at $12 \mathrm{dpi}$, and from 24,692.6 to 3,075.6 at 15 dpi (Supplemental Table S3.2). Phosphoglycerate kinase 1 (At3g12780), a phosphoglycerate kinase, decreased in FPKM value from 946 to 310 at $9 \mathrm{dpi}, 1,020$ to 221.5 at $12 \mathrm{dpi}$, and 1,473.6 to 171.1 at $15 \mathrm{dpi}$. Chaperonin 60 beta (At1g55490), an ATP-binding protein, decreased in FPKM value from 361.5 to 72 at 9 dpi, 245.5 to 28.3 at $12 \mathrm{dpi}$, and 389.5 to 18 at $15 \mathrm{dpi}$. Interestingly, the LHCII sub-pathway exhibited a different expression pattern in which gene expression increased at $15 \mathrm{dpi}$. In total, nearly $70 \%$ of genes in Photosystem I were downregulated (Fig. 3.5B). In addition, genes in photosystem II and the Calvin cycle increased in expression by $70 \%$ at 9 dpi and $80 \%$ at $12 \mathrm{dpi}$ and $15 \mathrm{dpi}$. A number of genes had moderately attenuated expression levels at $12 \mathrm{dpi}$ and $15 \mathrm{dpi}$. Collectively, these results indicate that photosynthesis-related pathways were gradually shut down after TSWV infection.

\section{Effects on the transcription of genes that regulate the cell wall}

The plant cell wall is composed of cellulose, hemicellulose, pectin, and soluble proteins, which together provide structural support and help maintain cell shape (Fig. 3.6A). As shown in Fig. 3.6, most DEGs related to cell wall regulation followed a similar expression pattern at $9 \mathrm{dpi}$, $12 \mathrm{dpi}$ and $15 \mathrm{dpi}$. Cell wall metabolic pathway genes were largely downregulated though some genes were upregulated. Statistical analyses showed that about 30\% of the DEGs related to cellulose synthesis were downregulated at $9 \mathrm{dpi}$, while $35 \%$ were downregulated at $12 \mathrm{dpi}$ and 45\% were downregulated at $15 \mathrm{dpi}$ (Supplemental Table S3.2). Other cell wall sub-pathways also had decreasing expression from $9 \mathrm{dpi}$ to $12 \mathrm{dpi}$, but were similar at $12 \mathrm{dpi}$ and $15 \mathrm{dpi}$. In addition, about $40 \%$ of genes related to cell wall degradation and modification were downregulated. Nearly $40 \%$ of genes in the AGP and pectin esterase subpathways were also downregulated (Supplemental Table S3.2). Among the downregulated genes, cellulose synthesis-related genes belonging to the cellulose synthase family were globally inhibited, including cellulose-synthesis like C2 (At5g22740) and cellulose-synthesis like C5 (at4g31590). AGP4 (At5g10430) and AGP7 (At5g65390), members of the cell wall arabinogalactan protein family, were also downregulated. The fold changes for AGP4 were 
1.7, 2.8 and 2.9 at $9 \mathrm{dpi}, 12 \mathrm{dpi}$ and $15 \mathrm{dpi}$, respectively. The fold changes for AGP7 were 1.6, 1.7, 1.6 at $9 \mathrm{dpi}, 12 \mathrm{dpi}$, and $15 \mathrm{dpi}$, respectively. Interestingly, among upregulated genes, several cell wall degradation enzymes were strongly induced (Table 3.1). The FPKM value of beta-1,3-glucanase 1 (At3g57260), a glucan degradation enzyme, increased from 13 to 1,459 at $9 \mathrm{dpi}$, from 229.6 to 2,461.4 at $12 \mathrm{dpi}$, and from 311 to 1,365 at $15 \mathrm{dpi}$. The corresponding fold changes were $6.8,3.4$, and 2.1 , respectively. This gene encodes pathogenesis-related (PR) proteins, which play roles in plant defence against pathogens (Edelbaum et al. 1991). The FPKM value of xyloglucosyl transferase (At4g30270) increased from 20.3 to 187 at 9 dpi, 22 to 172 at $12 \mathrm{dpi}$, and 16.7 to 272.4 at $15 \mathrm{dpi}$. At2g27500, another cellulase glycosyl hydrolase family 17 protein, was induced to a more moderate extent, exhibiting 1.5-, 1.3-, and 1.1-fold changes in expression at $9 \mathrm{dpi}, 12 \mathrm{dpi}$, and $15 \mathrm{dpi}$, respectively. These results indicate that cell wall metabolic activities declined at all three timepoints, which may impair cell structure and lead to metabolic deficiency.
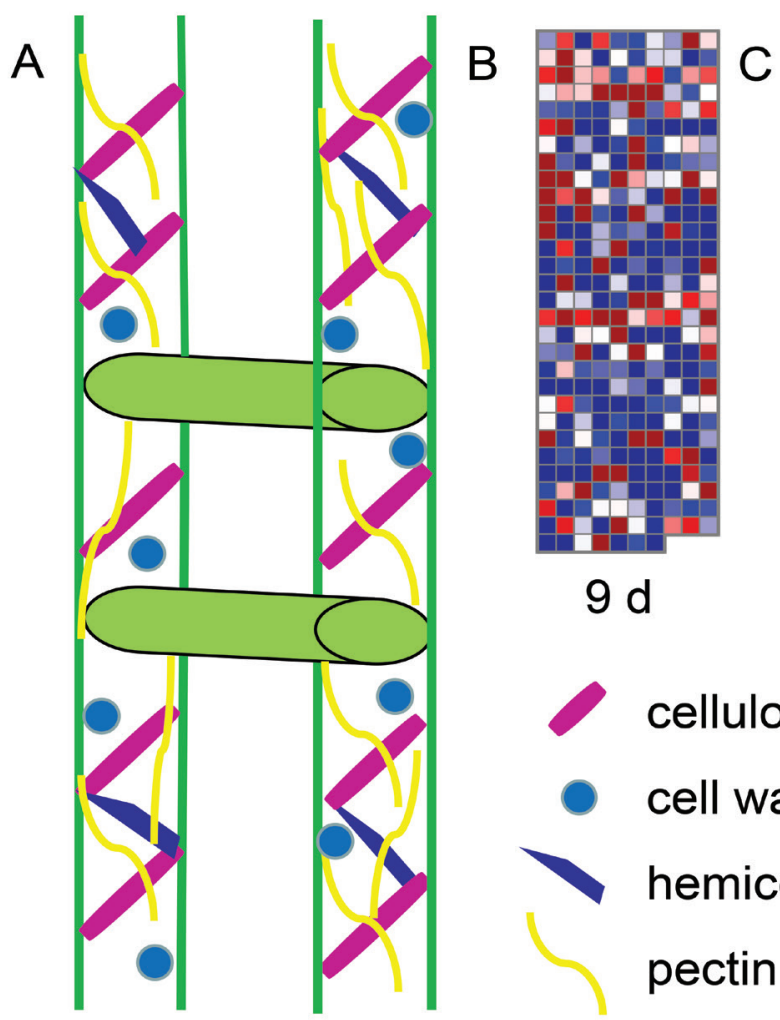

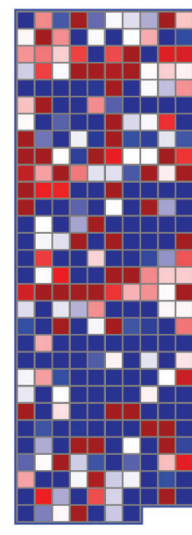

$12 \mathrm{~d}$

cellulose

cell wall protein

hemicellulose

pectin

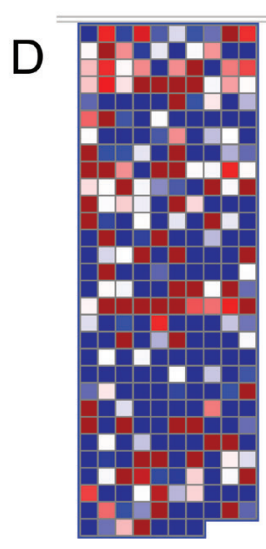

$15 d$

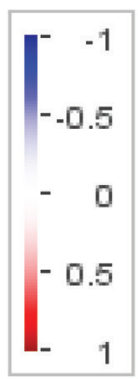

Figure 3.6 Differential expression of genes involved in cell wall synthesis and degradation. (A) depicts a model of the cell wall structure. (B), (C), and (D) are heatmap images of DEGs at $9 \mathrm{dpi}, 12 \mathrm{dpi}$, and $15 \mathrm{dpi}$. 
Table 3.1 Specific genes information in cell-wall-related pathway at 9, 12, and 15 days post inoculation, respectively.

\begin{tabular}{|c|c|c|c|c|c|c|}
\hline Gene ID & $9 d \log 2$ (fold change) & & $12 d \log 2$ (fold change) & & $15 d \log 2$ (fold change) & \\
\hline \multicolumn{7}{|c|}{ Cellulose synthesis } \\
\hline at2g24630 & -1.09763 & down & -1.24934 & down & -1.96844 & down \\
\hline at1g24070 & -1.73350 & down & -2.03652 & down & -1.65734 & down \\
\hline at5g16190 & -2.13355 & down & -1.99167 & down & -2.42748 & down \\
\hline at4g13410 & -1.93853 & down & -3.14795 & down & -3.07856 & down \\
\hline at4g31590 & -1.44560 & down & -1.92324 & down & -2.64772 & down \\
\hline at5g22740 & -1.09634 & down & -1.71385 & down & -1.94281 & down \\
\hline at5g03760 & -1.49523 & down & -2.66919 & down & -2.98299 & down \\
\hline at4g39350 & -1.01520 & down & -1.10480 & down & -0.80981 & \\
\hline at5g09870 & -1.14864 & down & -0.96883 & & -1.26934 & down \\
\hline \multicolumn{7}{|c|}{ Cell wall proteins } \\
\hline at1g68725 & -1.79001 & down & -1.86101 & down & -2.45361 & down \\
\hline at5g60490 & -1.07664 & down & -1.12628 & down & -1.97179 & down \\
\hline at3g52370 & -1.45045 & down & -2.39017 & down & -1.72635 & down \\
\hline at3g46550 & -1.02238 & down & -1.02835 & down & -1.87541 & down \\
\hline at5g06390 & -1.09031 & down & -1.40001 & down & -1.81449 & down \\
\hline at5g65390 & -1.56339 & down & -1.72986 & down & -1.58240 & down \\
\hline at3g11700 & -1.14563 & down & -1.35268 & down & -1.82186 & down \\
\hline at5g55730 & -1.26931 & down & -1.73121 & down & -1.98864 & down \\
\hline at2g04780 & -1.61843 & down & -2.08350 & down & -2.09742 & down \\
\hline at5g10430 & -1.69577 & down & -2.81834 & down & -2.94583 & down \\
\hline at1g03870 & -1.41825 & down & -1.33356 & down & -1.44982 & down \\
\hline at4g12730 & -1.60786 & down & -1.66258 & down & -1.38978 & down \\
\hline at5g44130 & -1.56750 & down & -2.12864 & down & -1.93975 & down \\
\hline at2g14890 & -1.30329 & down & -1.29551 & down & -1.92078 & down \\
\hline at2g45470 & -2.11281 & down & -2.71086 & down & -3.92062 & down \\
\hline \multicolumn{7}{|c|}{ Cell wall degradation enzymes } \\
\hline at5g62150 & 4.60138 & up & 4.64635 & up & 5.39635 & up \\
\hline at5g20950 & 1.64004 & up & 0.52836 & & 0.75202 & \\
\hline at4g30270 & 3.20631 & up & 2.96326 & up & 4.02860 & up \\
\hline at5g49360 & 1.12819 & up & 1.36208 & up & 1.52267 & up \\
\hline at3g54920 & 1.14378 & up & 0.25885 & & -0.74691 & \\
\hline at2g18660 & 4.15316 & up & 2.11973 & up & 2.45283 & up \\
\hline at2g45220 & 7.97132 & up & 6.48892 & up & 7.92552 & up \\
\hline at3g13790 & 2.38246 & up & 2.25579 & up & 3.00060 & up \\
\hline at1g21250 & 1.11909 & up & 0.79163 & & 0.81496 & \\
\hline
\end{tabular}




\section{Activation of ubiquitin-proteasome, autophagy, and plant defence responses}

Plants have evolved many defence responses to counteract TSWV infection (Zhu et al. 2019). In hormone signalling pathways, abscisic acid and ethylene influence plant resistance (Baccelli and Mauch-Mani 2016; Kachroo and Kachroo 2007; Mauch-Mani and Flors 2009). Accordingly, abscisic acid and ethylene were upregulated in the present study. At $15 \mathrm{dpi}$, their expression had increased by $\sim 40 \%$ (Fig. 3.7). Gibberellins and auxins had a balance of downregulated and upregulated genes.

PR proteins and heat-related pathways play an essential role in stress metabolism (Aparicio et al. 2005; Chandrasekaran and Chun 2016; Lu et al. 2003). We found that stimulus- and stress response-related pathways were significantly enriched with DEGs (Fig. 3.7). Almost 20\% of genes within such pathways were upregulated, while around $10 \%$ were downregulated.

It has been reported WRKY transcription factors play an important role in plant resistance (Eulgem 2006). WRKY8 influences the TMV-cg defence response by mediating both abscisic acid and ethylene signalling (Chen et al. 2013a). Based on our data, nearly 50\% of WRKY transcription factors were significantly upregulated, with a 1.1- to 7.3-fold increase in expression. A heatmap of defence process genes (Supplemental Fig. S3.2) validated these results. Collectively, the data showed that viral infection activated plant defence pathway at all three infection stages.

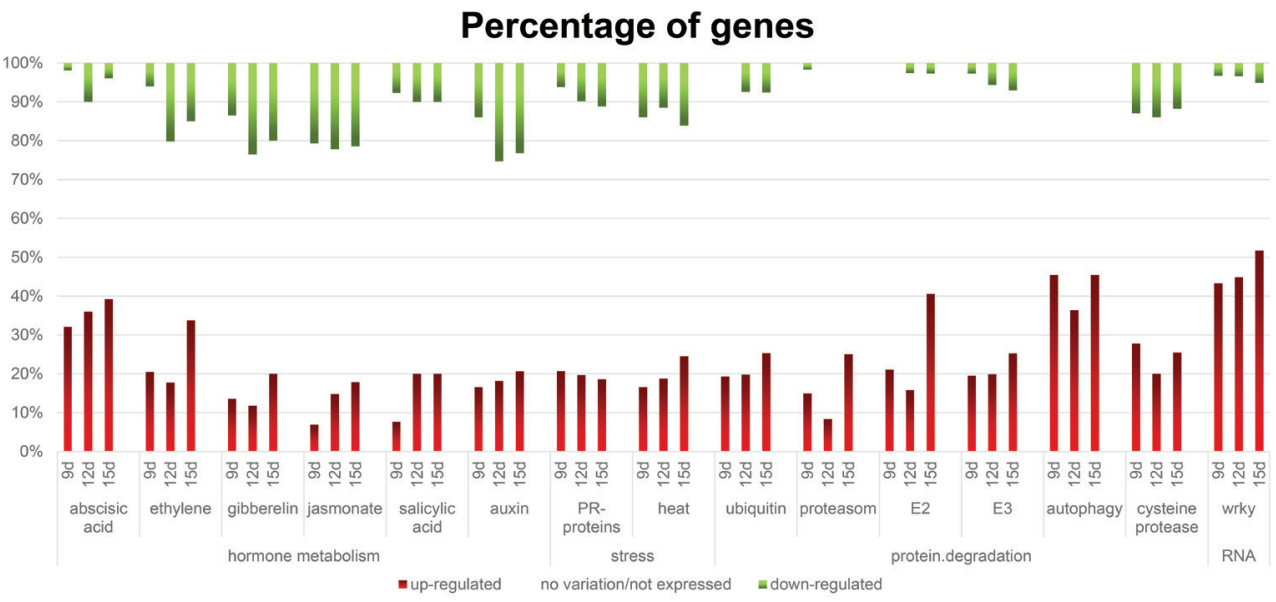

Figure. 3.7 Percentages of DEGs in plant defence and degradation pathways at 9 dpi, 12 dpi, and 15 dpi. Red indicates mostly upregulated genes in the corresponding pathway; green indicates mostly downregulated genes in the corresponding pathway. 
To resist viral spread and multiplication, plants implement protein degradation strategies to accelerate protein metabolism (Deng et al. 2017; Zeng et al. 2004). In the present study, we identified a remarkable change in autophagy-related genes: more than $40 \%$ of genes in the autophagy pathway were upregulated at $9 \mathrm{dpi}$ and $15 \mathrm{dpi}$, while a slightly lower percentage were upregulated at $12 \mathrm{dpi}$ (Fig. 3.7).

The ubiquitin-proteasome pathway is involved in the regulation of metabolic adaptation and the immune response (Furniss and Spoel 2015; Han et al. 2019). We found that $40 \%$ of E2 genes were upregulated at $15 \mathrm{dpi}$ (Fig. 3.7). Moreover, all proteins in the proteasome and cysteine protease degradation sub-pathways exhibited increased upregulation at $9 \mathrm{dpi}$ and $15 \mathrm{dpi}$. However, these sub-pathways had slightly decreased expression at $12 \mathrm{dpi}$ (Fig. 3.7). While some genes in the protein degradation pathway were downregulated, most were upregulated (Supplemental Fig. S3.2). These observations suggest that activation of the ubiquitin-proteasome, autophagy, and plant defence responses are hallmarks of cellular changes associated with TSWV infection.

\section{qRT-PCR validation of differentially expressed genes}

To validate the RNA-seq data, we compared gene expression profiles using qRT-PCR. Twenty genes from different pathways with altered expression patterns were selected for qRT-PCR analysis (Supplemental Fig. S3.5). qRT-PCR showed that the direction of change for all 20 genes was consistent with RNA-seq data (Fig. 3.8). For example, the qRT-PCR results showed that the fold increase in catalytic/ribulose-phosphate 3-epimerase expression in the Calvin cycle pathway was $1.2,1.8$, and 2.6 at $9 \mathrm{dpi}, 12 \mathrm{dp}$, and $15 \mathrm{dpi}$, consistent with the RNA-seq data. The qRT-PCR data for WRKY6 transcription factor were also consistent with the RNA-seq data, with fold increases in expression of 1.6, 2.6, and 3.6 at $9 \mathrm{dpi}, 12 \mathrm{dpi}$, and $15 \mathrm{dpi}$, respectively. Minor inconsistencies may have been caused by the lower sensitivity of qRT-PCR compared to RNA-seq. Nevertheless, qRT-PCR analyses broadly confirmed the direction of changes in expression detected by RNA-seq analyses, indicating that the RNA-seq data were reliable.

\section{Analysis of host cellular mRNA-derived leader sequences at the 5' end of viral mRNAs}

In addition to studying host gene expression in Arabidopsis during an infection with TSWV, the collected transcriptome also enabled to collect a large number of viral mRNAs for 
further studies on their 5' RNA leader sequences obtained by cap snatching. Unfortunately, only several reads in the range of 12-18 nt were identified, the others are rather too short or too long, while some are with polyadenine noisy signals. Thus, the data was insufficient for further analysis of capped leader sequences and we were unable to identify cytosolic mRNA sources from it.

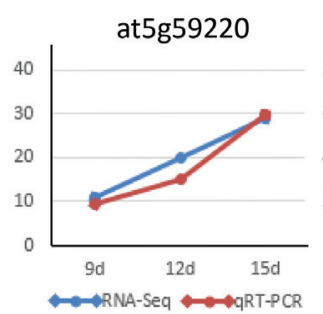

at5g17290

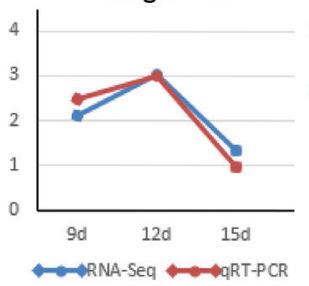

at5g35360

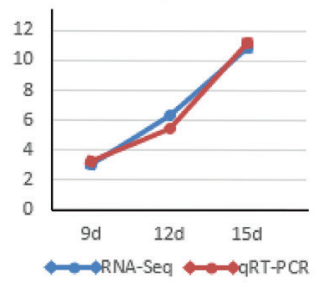

at2g04780

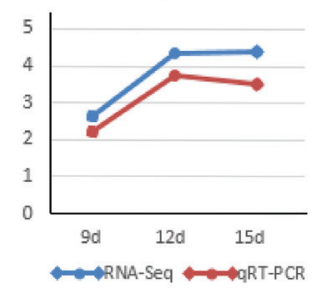

at3g44300

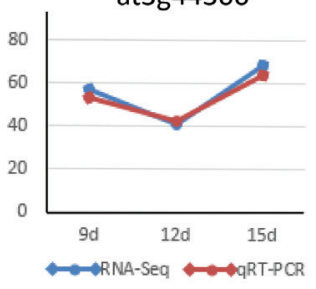

at1g62300

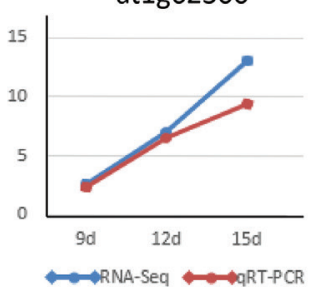

at2g30550

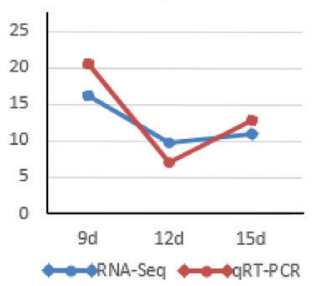

at1g32060

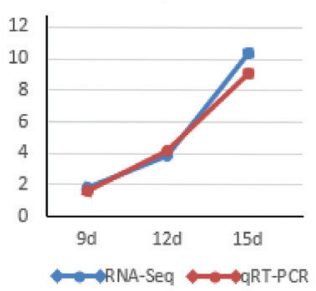

at5g43450

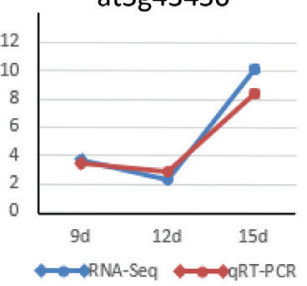

at2g30250

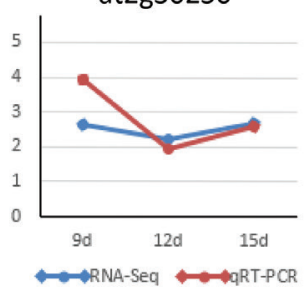

at5g03760

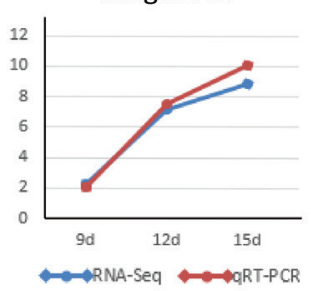

at2g05100

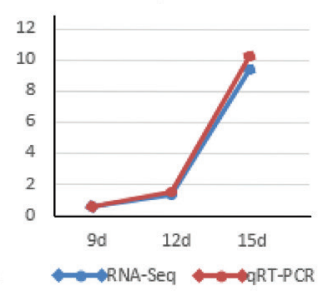

at3g07525

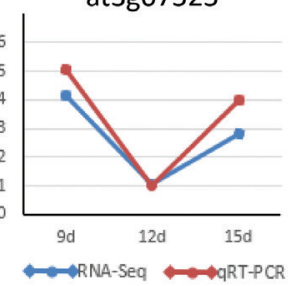

at2g46400

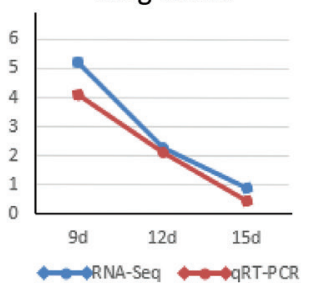

at3g24480

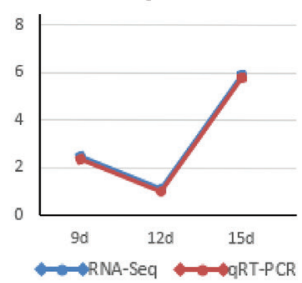

at3g55330

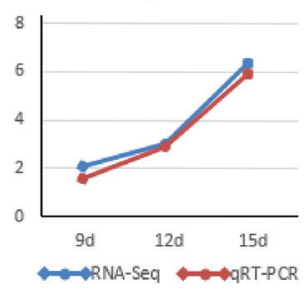

Figure 3.8 qRT-PCR validation of 16 differentially expressed genes from different pathways. The $X$-axis represents different timepoints and the $Y$ axis represents fold change. Red line represents the qRT-PCR results and blue line represents the RNA-seq results. 


\section{Discussion}

Viruses are obligate parasites that depend on the host's machinery to multiply and spread (Brodersen and Voinnet 2009; Dunoyer et al. 2004; Hyodo et al. 2013; Laliberte and Sanfacon 2010; Pallas and Garcia 2011; Sade et al. 2012; Satoh et al. 2010). Viruses may induce downregulation or upregulation of plant genes for their own benefit. By contrast, plant cells may sense viral invasion and initiate a series of defence responses (Dodds and Rathjen 2010; Mandadi and Scholthof 2013). In the present study, deep sequencing technology was used to characterize model plant $A$. thaliana dynamic transcriptome profiles during TSWV infection at different points over time, and to get hold on a large amount of viral mRNA reads to further study snatched RNA leader sequences at their 5 ' ends. Due to the low amount of viral mRNA reads collected containing non-viral heterogenous leader sequences in the range of 10-20 nt, this objective could not be further studied.

Photosynthesis is arguably the most important biological process on Earth, and plays import roles in plant growth, development, and defence against pathogens. Many plant viruses can impair photosynthesis to regulate plant growth and development, aiding viral infection. For instance, Potato virus $X$ (SPCP1 strain) changes the photosynthetic rate by regulating photosystem II and carbohydrate synthesis (Cueto-Ginzo et al. 2016). Geminivirus ${ }^{\beta c 1}$ protein attacks PsbP protein, an extrinsic subunit protein within the oxygen-evolving complex of photosystem II, and induces symptoms by disrupting the ultrastructure and function of chloroplasts (Bhattacharyya et al. 2015). In a previous study, transcriptome analyses of chlorotic Theobroma cacao leaves infected with the fungal pathogen Moniliophthora perniciosa revealed the downregulation of photosynthesis genes (Teixeira et al. 2014). Immunogold labeling has revealed Lily mottled virus (LMoV) coat protein localized to chloroplasts. The overaccumulation of this protein inhibits PSII activity, causing mottling symptoms (Zhang et al. 2019).

The studies described above showed correlations between photosystem damage and chlorotic symptoms; our results support these findings. We conclude that plant mottling and chlorotic symptoms in Arabidopsis may be caused by PSII system damage as a result of TSWV infection. Further research is required to identify the specific viral proteins and host factors contributing to this process. 
The plant cell wall is located outside the cell membrane and provides structural integrity and protection against biotic and abiotic stressors (Underwood 2012). It also plays a regulatory role in plant growth and development (Szymanski and Cosgrove 2009). Research on interactions between plant cell walls and viruses has revealed that cell wall-related genes are downregulated during infection. For example, Potato virus $Y$ infection inhibits xyloglucan CesA4 catalytic activity and increases xylan deposition to reduce cell wall integrity (Allie et al. 2014; Otulak-Koziel et al. 2018a; Otulak-Koziel et al. 2018b). In the present study, most cellulose synthesis-related genes were downregulated, indicating inhibition of the cellulose synthesis process. Furthermore, some cellulases and beta-1,4-glucanases were upregulated to promote cellulose and hemicellulose degradation. The disruption of cell walls might directly facilitate viral invasion and cell-to-cell movement. In addition, many cell wall-related genes are regulated by WRKY transcription factors, and viruses may recruit WRKYs to mediate cell wall metabolism (Chen et al. 2013b; Li et al. 2017; Sun et al. 2019; Zhu et al. 2018).

We found that viral infection regulates cell wall-related genes not only through transcription factors, but also by directly influencing genes involved in cell wall synthesis and degradation. Some specific degradation enzymes involved in cell wall degradation were highly induced, which might affect the recruitment of host factors (Horn et al. 2012). These enzymes catalyse cell wall disruption to facilitate viral infection and transport.

To counteract viral infection, plants may induce PR protein expression. PR is considered the first line of defence against pathogens (Breen et al. 2016; Reiss 1998; Wang et al. 2018). Tobacco mosaic virus infection induces the expression of PR-1, PR-4, and PR-5 proteins (Wang et al. 2018). As discussed previously, WRKYs manipulate gene expression to initiate the defence response under biotic stress (Eulgem 2006; Eulgem and Somssich 2007; Gallou et al. 2012; Ryu et al. 2006; Yamada et al. 2007). Under TSWV infection, these genes were massively upregulated to manipulate the expression of related genes, such as those for PR proteins and cell wall-related proteins. The ubiquitin and autophagy pathways play a role in protein degradation, and many studies have shown that these pathways are involved in host resistance to viral infection (Verchot 2016). The E2 ligase family can mediate plant immunityassociated reactive oxygen species (ROS) and suppress multiple immunity-associated PCDs in N. benthamiana (Zhou and Zeng 2017). TYLCV and Turnip mosaic virus (TuMV) infections induce increased expression of autophagy pathway-related genes to inhibit viral infection (Hafren et al. 2018; Miozzi et al. 2014). Plant transcriptome rearrangement is a complex 


\section{Chapter 3}

process during viral infection, and includes manipulation of photosystems, cell wall-related metabolism, hormones, and the defence responses. Analyses of this phenomenon at the system, pathway, or even gene-specific level can provide deeper insight into TSWV-host interactions and symptom development.

\section{Acknowledgements}

This work was supported by grants from the National Natural Science Foundation of China (31630062 and 31870143), the Fundamental Research Funds for the Central Universities (JCQY201904), and the Youth Science and Technology Innovation Program (to X. Tao). We thank Dr. Harm Nijveen (Wageningen University) for the help in capped leader sequence analysis of RNA-seq data. 


\section{Supplemental data}

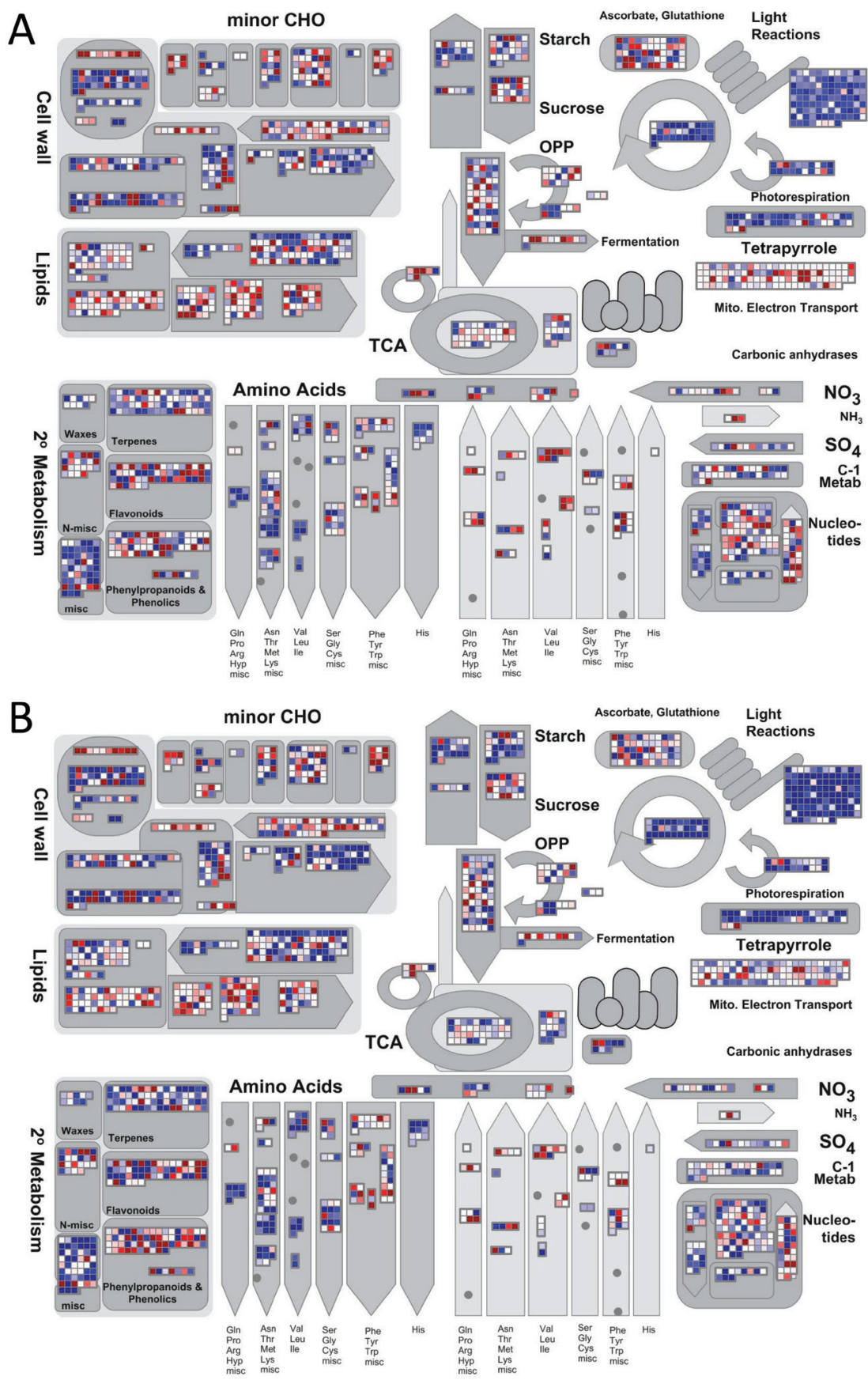



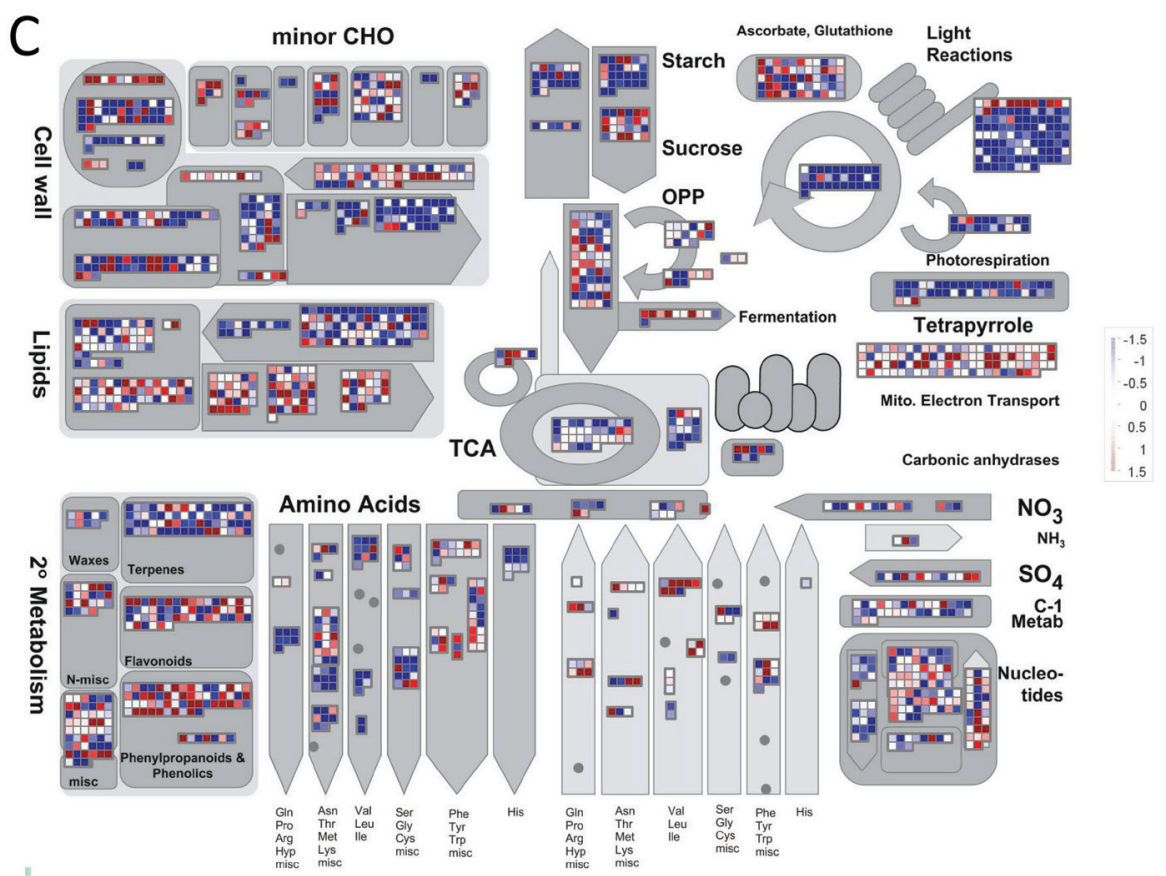

Supplemental Figure S3.1 Heatmap of metabolic changes at (A) 9 dpi, (B) 12 dpi, and (C) 15 dpi. Blue represents downregulated genes and red represents upregulated genes. Log2 (fold change) values from -1.5 to 1.5 is used as the threshold.
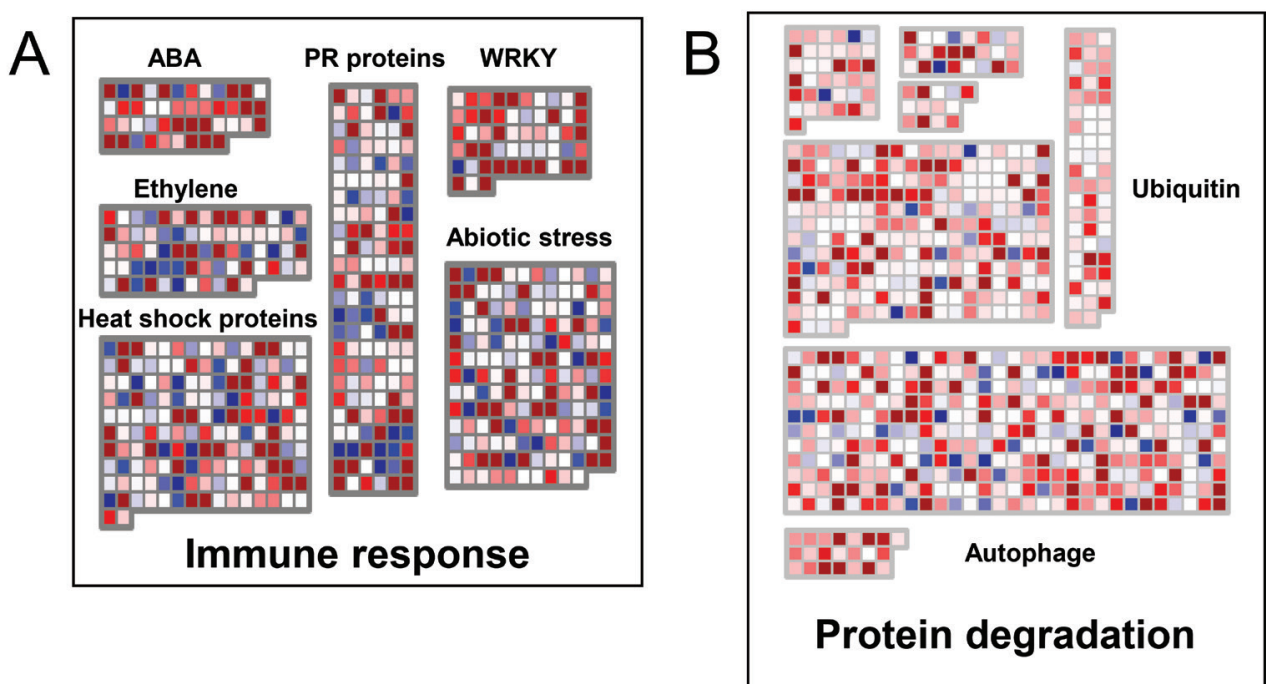

Supplemental Figure S3.2 Heat map of DEGs in pathways related to (A) plant defence and (B) degradation. Log2 values (fold changes) at $9 \mathrm{dpi}, 12 \mathrm{dpi}$, and $15 \mathrm{dpi}$ were averaged. Blue represents downregulated genes and red represents upregulated genes. Log2 (fold change) from -1.5 to 1.5 is used as the threshold. 
Supplemental Table S3.1 Mapping rates of 18 sequencing data libraries to the Arabidopsis thaliana genome.

Supplemental Table S3.2 All transcripts assembled from raw data are listed at 9 dpi, 12 dpi, and 15 dpi. Gene annotation and FPKM values are assembled and Log2 (fold change) values are calculated.

Supplemental Table S3.3 List of DEGs at 9 dpi, 12 dpi, and 15 dpi. FPKM values and Log2 (fold change) values are listed.

Supplemental Table S3.4 List of DEGs in photosynthesis pathways at 9 dpi, 12 dpi, and 15 dpi. Log2 (fold change) values are listed.

Supplemental Table S3.5 qRT-PCR primers used in this study.

These supplemental tables can be found at the link: https://apsjournals.apsnet.org/doi/suppl/10.1094/ PHYTO-06-19-0199-FI 



\section{Chapter 4}

\section{Cytoplasmic sources of capped RNA for genome transcription initiation of cytoplasmic replicating, segmented negative strand RNA viruses}

Min Xu, Magdalena J. Mazur, Nigel Gulickx, Xiaorong Tao and Richard J. M. Kormelink

This chapter will be submitted in a slightly modified version as:

Min Xu, Magdalena J. Mazur, Nigel Gulickx, Xiaorong Tao and Richard Kormelink (2021). Cytoplasmic sources of capped RNA for genome transcription initiation of cytoplasmic-replicating, segmented negative strand RNA viruses. 


\section{Abstract}

Most viruses with a segmented, negative strand (-) RNA genome initiate transcription by capsnatching. The source from which the cytoplasmic-replicating RNA viruses snatch cappedRNA leader sequences has still remained elusive. Earlier reports have pointed towards cytoplasmic RNA processing bodies, although several questions relating to this issue still have remained unsolved. Here, several plant- and animal-infecting members of the order Bunyavirales, in casu Tomato spotted wilt virus (TSWV), Sin nombre virus (SNV), CrimeanCongo haemorrhagic fever virus (CCHFV) and Schmallenberg virus (SBV) were investigated. Nucleocapsid ( $\mathrm{N}$ ) protein, transiently expressed in plant and animal cells, localized to RNA processing $(\mathrm{P})$ bodies and stress granules (SGs). Also, during a viral infection of plant and animal cells with TSWV and SBV, respectively, their N proteins extensively colocalized with both $P$ body and SG markers. Silencing of the gene encoding $P$ body enzymatic element DECAPPING 5 (DCP5) increased TSWV accumulation, whereas silencing of NMD factor gene UPF1 reduced TSWV accumulation. Silencing the SG components Ras GTPase-activating protein-binding protein 1 (G3BP1) or Polyadenylate-binding protein RBP47 (Rbp47) led to a higher accumulation level of TSWV. Silencing the SG-resident translation initiation factor eIF4A reduced TSWV accumulation, whereas silencing of elF4E, a SG-resident translation factor but also an important factor in nuclear mRNA export, increased TSWV accumulation. Concomitant silencing of both P body and SG components (DCP5 and G3BP1) in plant cells, only led to a slight increase in viral titers. Both TSWV and Rice stripe virus (RSV) N proteins also co-localized with RanGAP2 in the perinuclear region, and partly in the nucleus when co-expressed with RanGAP2 containing a nuclear-localization-signal. Upon silencing of the RanGAP 1 and 2 in plant tissues, TSWV titers decreased. The results altogether implicate a role of additional foci/RNA granules, besides $P$ bodies, in the transcription of these viruses. The findings are discussed in light of putative cytoplasmic sources for capped-RNA leader sequences for cap-snatching by cytoplasmic-replicating negative strand RNA viruses (NSVs). 


\section{Introduction}

The RNA dependent RNA polymerase (RdRp) from most segmented, negative-strand (-) RNA viruses lacks a methyltransferase activity that normally is needed to provide a $5^{\prime}$ terminal $\mathrm{m}^{7} \mathrm{G}$ cap on viral RNA transcripts to support their translation (Decroly et al. 2011). To circumvent this problem, these viruses employ cap-snatching, a process during which capped-RNA leader sequences are cleaved from host cellular messengers and used to align on the $3^{\prime}$ end of viral RNA segments to prime transcription. In recent years, 3-D folding structures have been resolved of the viral RdRp complex from Influenza virus and several other viruses that employ cap-snatching, which has revealed quite some structural similarities and supports a highly conserved mechanism of genome transcription initiation (Reguera et al. 2016; Reich et al. 2014). For the nuclear replicating Influenza virus, the RdRp complex consists of three subunits called PA, PB1 and PB2. Whereas PA contains the endonuclease domain (Yuan et al. 2009) and PB1 the catalytic core domain of the RNA polymerase, PB2 contains the cap-binding domain (Guilligay et al. 2008; Stevaert and Naesens 2016). In contrast, for all cytoplasmic replicating segmented, (-)RNA viruses employing cap-snatching, i.e. members of the order Bunyavirales, the RdRp consists of a single protein that holds an endonuclease domain in the $\mathrm{N}$ terminus and six polymerase motifs, characteristic of the RdRp of (-) RNA viruses, in the central region (Reguera et al. 2010; Zhao et al. 2019). However, the rest of the large RdRp protein, ranging in size from $\sim 250 \mathrm{kDa}$ to $\sim 460 \mathrm{kDa}$ between these members, so far is functionally uncharacterized, partly due to the absence of sequence homology with other proteins. A cap-binding domain (CBD), which, according to alignments with the Influenza virus polymerase units, was suspected to map to the C-terminal domain of their RdRps, has not been found (Reguera et al. 2010). Despite an overall structural homology more recently found between the CBD of Influenza polymerase and the C-terminal domain of the RdRp from those of LaCrosse virus (LACV), Rift valley fever virus (RVFV) and the California Academy of Sciences Virus (CASV) (Gerlach et al. 2015; Gogrefe et al. 2019; Vogel et al. 2019), their low affinity for cap structures does not explain how they are able to compete with high-affinity cellular cap-binding proteins. Furthermore, a C-terminal part of the Reptarenavirus (Arenaviridae) polymerase, with structural resemblance to the cap-binding domain of Influenza virus, turned out to present a degenerate cap-binding domain due to the absence of a typical structural sandwich of two aromatic residues, and failure to biochemically detect capbinding (Rosenthal et al. 2017). 
Although these findings do not entirely rule out the possibility for the RdRp proteins of these viruses to harbor a $\mathrm{CBD}$, they do raise the question whether or not another viral protein that is also needed for transcription-replication of these viruses, e.g. the nucleocapsid (N) protein, could harbor such a domain, which could possibly act in concert with RdRp to fulfil cap-snatching.

In the past two decades, studies have demonstrated that (multiple) base complementarity of the 3 '-end residues of capped-RNA leader molecules to the viral RNA genome template promotes their usage as primers during viral genome transcription initiation. This feature has been observed with several plant- and animal infecting viruses (Cheng and Mir 2012; Duijsings et al. 1999, 2001; Geerts-Dimitriadou et al. 2011a; Geerts-Dimitriadou et al. 2011b; Liu et al. 2016; van Knippenberg et al. 2002; van Knippenberg et al. 2005; Yao et al. 2012) and supports the idea that the mechanistic model for cap -snatching is likely generic to all these viruses. From all segmented (-)ssRNA viruses that employ capsnatching, Influenza virus is the only one that replicates in the nucleus where its viral RNA polymerase complex interacts with the large subunit of RNA Polymerase II (Pol II) via its C-terminal domain (Chan et al. 2006; Engelhardt et al. 2005). Being confined to RNA Pol II transcription sites, the Influenza virus has direct access to (a continuous supply of) cellular capped pre-mRNAs from which capped-RNA leaders are snatched to initiate viral genome transcription. However, where and how the cytoplasmic replicating (-)ssRNA viruses snatch capped-RNA leaders still remains somewhat elusive. It is tempting to assume that the cytoplasmic replicating, segmented (-)ssRNA viruses similarly use a costeffective strategy for cap snatching and confine to specific areas in the cytoplasm enriched with mRNA as source for capped-RNA leaders (Tsai and Lloyd 2014b). Candidates for these are two major cytoplasmic RNA granules i.e., RNA processing bodies (PBs) and stress granules (SGs)(Reineke and Lloyd 2013b). Although both act as storage sites for "silenced" mRNA and segregate mRNA away from the active (polysomal) translational machinery, PB and SG are distinct in their function and composition (Anderson and Kedersha 2008; Chantarachot and Bailey-Serres 2018; Loschi et al. 2009). P bodies are constantly present in the cell and play a major role in cellular RNA turnover as they contain the RNA decay machinery (waste bin) of the cell. SGs on the other hand are dynamic and transient foci enriched in (functional) mRNAs stalled in their translation and complexed with translation initiation factors, poly (A) binding protein (PABP), and the $40 \mathrm{~S}$ ribosomal subunit. Under conditions of stress (e.g. induced by arsenite, heat or during viral infection), SGs often localize in close proximity of and even physically interact with $\mathrm{P}$ bodies in mammalian 
cells, with several proteins thought to shuttle between both organelles, as observed by the presence of proteins like elF4E, TTP, and Ago2 in both organelles (Parker and Sheth 2007). Meanwhile evidence is accumulating that RNA granules, in specific SG, are also involved in the sensing of viral infections and mounting of an antiviral response, and this explains why many different viruses are observed to antagonize the formation of these granules in order to establish a viral infection (Burgui et al. 2007; Linero et al. 2011; Tsai and Lloyd 2014b).

Earlier observations made on the Sin Nombre (SNV) hantavirus pointed towards RNA processing $(\mathrm{P})$ bodies as the source for capped-RNA leaders. Not only its nucleocapsid $(\mathrm{N})$ protein co-localizes with the $P$ body marker protein De-capping 1 enzyme (DCP1) but the virus also seems to preferably snatch cap leader sequences from transcripts that target to $P$ bodies via the nonsense-mediated decay (NMD) pathway, a cellular surveillance mechanism that detects mRNA transcripts containing a premature termination codon (PTC) (Cheng and Mir 2012; Mir et al. 2008b). However, rates of cap-snatching/viral transcription for SNV using PTC containing transcripts are similar in normal and P body deficient cells and argues against $P$ bodies being the major source for capped-RNA leader molecules.

This raises the question whether $P$ bodies present the (first and sole?) source for capped RNA leaders to support cap snatching as initially postulated by Mir et al (Mir et al. 2008b). and whether or not other cytoplasmic RNA granules or foci present an (additional) source for capped-RNA leaders. Stress granules, due to their intimate relation with PBs, present a major candidate for these, but also $U$ bodies could present a source. $U$ bodies have been discovered not long ago and present RNP structures that contain Uridine-rich, capped small nuclear (sn) RNAs that mature in the cytoplasm and also associate with P bodies (Liu and Gall 2007), but finally localize in the nucleus where they play a key role in (RNA Pol II complex- mediated) pre-mRNA processing. Support for $U$ bodies as being another source comes from two recent studies on Influenza virus transcription where snRNAs presented a preferred source for capped-RNA leaders (Gu et al. 2015; Koppstein et al. 2015) (Chapter 2).

Considering the highly conserved nature of cap-snatching and the failure so far to proof a fully functional and primary cap-binding domain in the viral RNA polymerase from all cytoplasmic replicating segmented (-)RNA viruses, this study embarked from a concept in which the $\mathrm{N}$ protein is postulated to play a role in the recognition of host mRNAs by (in) directly mediating the binding to $5^{\prime}$ cap-structures. This idea is supported by the observations 
that 1 ) the SNV hantavirus $N$ protein shows affinity to the $5^{\prime}$ cap of mRNAs and plays an important role in the initial stages of translation initiation (Haque and Mir 2010; Mir et al. 2008b; Panganiban and Mir 2009), 2) the Junin (JUNV), Tacaribe (TCRV) and Pichinde (PICV) arenavirus $\mathrm{N}$ proteins are able to interact with a 7 methyl-guanosine (cap) (Linero et al. 2013) and 3) the Crimean-congo haemorrhagic fever (CCHFV) nairovirus $\mathrm{N}$ protein and TSWV tospovirus $\mathrm{N}$ protein enhance translation of viral mRNAs (Geerts-Dimitriadou et al. 2012; Jeeva et al. 2017a; Jeeva et al. 2017b). If this is true, the (indirect) affinity to 5' cap structures will direct and concentrate these $\mathrm{N}$ proteins at cytoplasmic foci enriched for capped-RNA, as previously observed with SNV (Mir et al. 2008b). In light of all these findings a recent study on TSWV has also shown its $\mathrm{N}$ protein to (partially) co-localize with $\mathrm{P}$ bodies and that the $\mathrm{P}$ body de-capping machinery affects TSWV accumulation. When taking out a $\mathrm{P}$ body de-capping element, TSWV accumulates to higher levels and this accumulation was counteracted by heat treatment. However, whether $\mathrm{P}$ bodies function as a cap donor source for TSWV was not investigated. In addition, the influence of heat treatment in this process was observed, but no further investigation was executed on heat-related stress granule formation and a possible role as source for TSWV cap-snatching.

In this study the $\mathrm{N}$ proteins from several plant- and animal infecting segmented (-) RNA viruses have been localized in situ, relative to markers for cytoplasmic RNA granules, to analyse whether all these proteins exhibit similar localization behaviour relevant to $P$ bodies and besides $\mathrm{P}$ body might localize to other foci enriched for capped RNA. Irrespective of their origin from a plant- or animal-infecting virus, expression in plant- and animal cells consistently revealed these $\mathrm{N}$ proteins to localize with $\mathrm{P}$ bodies and SG. Other than these RNA granules, the plant virus $\mathrm{N}$ proteins also localized to the perinuclear region, partly overlapping with RanGAP, an important nucleocytoplasmic transport factor.

\section{Materials and methods}

\section{Cloning of the constructs}

All molecular standard techniques were performed using protocols as described (Sambrook \& Russell, 2001)Primers used in the study are listed in the Supplementary Table 4.1 and were synthetized by Integrated DNA Technologies. Constructs made and used in this study were verified by sequence analysis (Eurofins Genomics, Germany). 
For the generation of fluorophore-fusion constructs, first eCFP, eGFP, eYFP and mRFP coding sequences were amplified using primers containing Sacll restriction sites (Supplementary Table 4.1) and subsequently cloned into Sacll pre-digested pcDNA-DEST40 (Thermo Fischer Scientific), resulting in pcDNA-DEST40-eCFP, pcDNA-DEST40-eGFP, pcDNA-DEST40-eYFP and pcDNA-DEST40-mRFP.

TSWV orthotospovirus $\mathrm{N}$ and Rice stripe tenuvirus (RSV) $\mathrm{N}$ sequences were cloned from infected plant material. Crimean-Congo hemorhagic fever nairovirus (CCHFV) N, Sin nombre hantavirus (SNV) N, European mountain ash ringspot-associated virus (EMARaV) $\mathrm{N}$ were kindly provided by dr. J. Barr, A. Panganiban and C. Buttner, respectively. $\mathrm{N}$ gene sequences were re-amplified with primers containing attB1 and attB2 recombination sites (Supplementary Table 4.1) and recombined into Gateway vector pDONR207 (Thermo Fischer Scientific) using BP Clonase II (Thermo Fischer Scientific). To obtain CMV driven fluorophore-tagged constructs for expression in mammalian cells, $\mathrm{N}$ gene constructs were transferred from the entry clones into either pcDNA-DEST40-eCFP, pcDNA-DEST40-eGFP, pcDNA-DEST40-eYFP or pcDNA-DEST40-mRFP by using Gateway LR clonase II (Thermo Fischer Scientific, Country of supplier). For expression in plant tissues, pDONR207 entry constructs harboring the plant virus $\mathrm{N}$ genes of TSWV, RSV and EMARaV were recombined into destination vector pK2GW7 by LR Clonase Enzyme Mix (Invitrogen, country of supplier). Mammalian RNA granules markers Hs decapping 1(HsDCP1), HsCaprin-1 and HsG3BP1 were kindly provided by dr. N. Kedersha. Plant P body marker RFP-DCP1 was kindly provided by dr. A. Maizel. Tandem zinc finger protein 1 fused with RFP (TZF1-RFP) and G3BP1-RFP were kindly provided by dr. Björn Krenz.

In order to generate dsRNA for gene silencing assays in animal cells, pGEM ${ }^{\circledR}-T^{T}$ Easy (Promega) containing T7 and Sp6 promotor sequences flanking the multiple cloning site, was used to generate gene constructs of the P body marker HsDCP1a. The coding sequence of HsDCP1a was amplified by $\mathrm{Q}^{\circledR}{ }^{\circledR}$ High-Fidelity DNA Polymerase (New England Biolabs) following the manufacturer's protocols (Supplementary Table 4.1). Fragments obtained were subsequently tailed with A-residues using GoTaq ${ }^{\circledR}$ DNA Polymerase according to the manufacturer's protocol (Promega), prior to ligation into pGEM-T easy vector using the pGEM $^{\circledR}$-T Easy Vector Systems kit (Promega). 


\section{Cell culture, transfection and virus infection}

HeLa, HeLa G3BP KO and African green monkey kidney Vero E6 cells (ATCC CRI-1586) were cultured at $37^{\circ} \mathrm{C}$ with $5 \% \mathrm{CO} 2$ in Dulbecco's Modified Eagle Medium (DMEM, Gibco) supplemented with $10 \%$ fetal bovin serum (FBS, Fisher Inv.) and penicillin and streptomycin (Sigma-Aldrich) at the final concentration of $100 \mathrm{U} / \mathrm{ml}$ and $100 \mu \mathrm{g} / \mathrm{ml}$, respectively. Cells grown in a pre-seeded Nunc ${ }^{\mathrm{TM}}$ Lab-Tek $^{\mathrm{TM}}$ II 8-Chamber Slide ${ }^{\mathrm{TM}}$ were transfected with $250 \mathrm{ng}$ DNA (per construct) by using TransIT ${ }^{\circledR}$-LT1 Transfection Reagent (Mirus Bio) in Opti-MEM serum (Gibco) following the manufacturer's protocols. Schmallenberg orthobunyavirus (SBV) infection of animal cells was performed at a MOI of 2.2. Two hours post-virus infection, the cells were washed with PBS (Gibco) and the medium was replaced with fresh DMEM.

\section{Immunostaining}

Cells were fixed for 10 minutes in $4 \%$ paraformaldehyde in PBS, followed by permeabilization for 10 minutes at RT in $0.1 \%$ sodium dodecyl sulphate (SDS) in PBS. After a PBS wash for three times, cells were blocked by incubation in 5\% FBS for $30 \mathrm{~min}$. To immunolocalize SBV N, primary monoclonal mouse antibody against SBV-N (1:100, kindly provided by dr. K. Wernike), was used in combination with secondary antibody goat anti-mouse-Alexa Fluor 488 (1:1000; Thermo Fisher Scientific) or goat anti-mouse-Alexa Fluor 633 (1:1000; Thermo Fisher Scientific). In order to induce SGs, cells were treated with $0.5 \mathrm{mM}$ sodium arsenite for 1 hour prior to the immunostaining experiment and washed three times with PBS. The primary antibodies against elF3 (polyclonal goat, 1:500; Santa Cruz Biotechnology) were used in combination with secondary antibody donkey anti-goat-Qdot565 (1:50; Thermo Fisher Scientific) or donkey anti-goat-Alexa 568 (1:2000; Thermo Fisher Scientific). Proteins were visualized in mammalian cells $24 \mathrm{hrs}$ post transfection or post infection, unless stated otherwise. Microscopical analysis was performed using a confocal laser scanning microscope (Zeiss LSM 510-META). Images were taken using the Plan-Apachromat 63x objective, with the pinhole kept at 1 Airy unit throughout all experiments. The obtained images were processed using ImageJ (ImageJ, $\mathrm{NIH}$ ).

\section{Plant material, transient expression and virus inoculation}

Nicotiana benthamiana were kept under greenhouse conditions $\left(24^{\circ} \mathrm{C}\right.$, during a regime of $16 \mathrm{~h}$ light/8 $\mathrm{h}$ dark per day). Six- to eight-week-old plants of $N$. benthamiana were used for all transient expression analyses and virus inoculations. 
$\mathrm{N}$ gene constructs and granule markers were transformed into Agrobacterium tumefaciens GV3101. Cells harboring different constructs were grown overnight at $28^{\circ} \mathrm{C}$. After centrifugation, the pellet was resuspended and treated with infiltration buffer $(10 \mathrm{mM}$ $\mathrm{MgCl} 2,10 \mathrm{mM}$ MES, $\mathrm{pH}$ 5.9, and $150 \mu \mathrm{M}$ acetosyringone) for $3 \mathrm{~h}$ at room temperature. $N$. benthamiana leaves were infiltrated with combinations of suspensions containing a final optical density at $600 \mathrm{~nm}$ (OD600) of 0.5 per construct. TSWV infection of plants was established by inoculation of virus from source inoculum using inoculation buffer $(0.01 \mathrm{M}$ $\mathrm{Na} 2 \mathrm{HPO} 4 . \mathrm{H} 2 \mathrm{O}, 0.01 \mathrm{M} \mathrm{Na} 2 \mathrm{H} 2 \mathrm{PO} 4.2 \mathrm{H} 2 \mathrm{O}, \mathrm{pH} 7.5)$, 5 days before infiltration with marker gene constructs. For heat shock treatment, plants were incubated at $37^{\circ} \mathrm{C}$ for $45 \mathrm{~min}$ followed by 10 min under dark condition at room temperature.

For virus-induced gene silencing (VIGS), $N$. benthamiana plants were infiltrated with suspensions of $A$. tumefaciens containing an infectious TRV clone with sequences from the host gene to be targeted. During VIGS silencing of host genes, a TRV-GUS gene construct was used as a negative control and TRV-phytoene desaturase (TRV-PDS) as positive control. Approximately 3 weeks later, when plants subjected to TRV-PDS exhibited chlorophyl bleaching in the top leaves, plants were superimposed with a TSWV infection by mechanical inoculation with virus inoculum, or by agroinfiltration with the TSWV infectious clones (Feng et al., 2020) for the L, M and S-GFP reporter constructs (Feng et al.). For heat treatment, plants were incubated at $37^{\circ} \mathrm{C}$ for 8 hours prior to fluorescence microscopy.

\section{Confocal microscopy and green fluorescence microscopy observation}

Agrobacteria-infiltrated leaf samples were harvest at $48 \mathrm{~h}$ post infiltration and were observed under Zeiss (Jena, Germany) LSM 510-META 18 confocal laser scanning microscope with a $\times 60,1.3$-numerical aperture, oil-corrected objective. Samples infected by mechanical inoculation with TSWV or via agroinfiltration of TSWV genome replicons were harvest at $60-72 \mathrm{~h}$ post infiltration and were analysed by Olympus fluorescence microscopy. GFP excitation was performed at $488 \mathrm{~nm}$ and emissions captured at 500-530 nm. For mRFP excitation and emission $543 \mathrm{~nm}$ and 560-615 nm were used, respectively. For CFP this was $405 \mathrm{~nm}$ and 418-480 nm, respectively. Images were processed using a Zeiss $2010 \mathrm{CLSM}$ and Image J viewer. 


\section{Quantification and Statistical analysis}

To quantify the number and size of the granules, Image Analyse Particle Analyser was used. To correct for background noise, a lower cut off for the size of the granules of 0.04 $\mu \mathrm{m}$ was applied. As close proximity of granules might result in a creation of one larger granule, an upper cut off of $6 \mu \mathrm{m}$ was applied. For statistical analyses, at least 10 cells were collected for each treatment. All statistical analyses were performed using IBM SPSS Statistic 25 software. Means were compared by one-way ANOVA with Tukey post-hoc test ( $p$-value of 0.05 ). In cases when the tested data sets did not fulfil the assumptions of ANOVA, the Kruskal-Wallis test was performed with a p-value of 0.05 . ${ }^{*}$-value $<0.05, * *$ p-value $\leq 0.01, * * *$ p-value $\leq 0.001$.

\section{RNA isolation and cDNA synthesis}

Total RNA was isolated from mammalian cells using TRIzol reagent protocol (Invitrogen). Briefly, $500 \mu \mathrm{L}$ TRIzol was added to $100 \mu \mathrm{L}$ cells lysed in passive lysis buffer (Promega). The samples were incubated at room temperature for 0.5 hour, accompanied with regular vertexing every 10 minutes. Afterwards, $100 \mu \mathrm{L}$ chloroform was added and mixed by inversion. The mixture was centrifuged at 10,000 RPM for 15 minutes at $4^{\circ} \mathrm{C}$ and $300 \mu \mathrm{L}$ of the upper (aqueous) layer was transferred to a new Eppendorf tube containing 300 $\mu \mathrm{L}$ isopropanol and centrifuged at 10,000 RPM for 15 minutes at $4^{\circ} \mathrm{C}$. The pellet was washed with $1 \mathrm{~mL} 70 \%$ ethanol and centrifuged at 9000 RPM for 5 minutes at $4^{\circ} \mathrm{C}$. The air-dried RNA pellet was dissolved in $20 \mu \mathrm{L}$ Milli-Q by incubation for 2 minutes at $95^{\circ} \mathrm{C}$. The concentration of RNA was measured using the Nanodrop UV-photo spectrometer. The RNA was treated with TURBO DNase for 30 minutes at $37^{\circ} \mathrm{C}$ (TURBO DNA-free ${ }^{\mathrm{TM}} \mathrm{Kit}$, Invitrogen) followed by a DNase inactivation step. First strand cDNA synthesis was performed on 500 ng total RNA per reaction using RT M-MLV (Promega) and random hexamers following the manufacturer's protocol.

Total RNA was isolated from plant materials using the same protocol. To this end, $0.1 \mathrm{~g}$ leaves were collected from plants and ground in liquid nitrogen followed with TRIzol incubation and extraction as described. 


\section{Quantitative real-time PCR (qRT-PCR)}

After reverse transcription, first strand cDNA was diluted five times prior to further analyses. Primers to genes were designed for quantitative analyses of RNA expression levels (see supplementary table 4.1). The qRT-PCR was performed in an ABI 7500 Real-Time PCR system (Life Technologies). Actin 2 and EF1a served as internal controls to normalize the RNA levels of target gene expression between samples, using a relative quantification method.

\section{Protein extraction and immunoblotting}

After observing fluorescence signals by fluorescence microscopy, leaves were collected and either half used for qRT-PCR and western blot analyses, respectively. For protein extraction, $0.1 \mathrm{~g}$. of $N$. benthamiana-infiltrated leaf sample was grinded in 200ul PBS buffer and centrifuged for $10 \mathrm{~min}$. at $12000 \mathrm{~g}$ and $4^{\circ}$. Supernatant was collected and boiled for 10 min. at $95^{\circ} \mathrm{C}$ in protein loading buffer ( $50 \mathrm{mM}$ Tris- $\mathrm{HCl}$ pH 6.8, $6 \%$ glycerol, $2 \% \mathrm{SDS}, 0.3 \mathrm{mg} /$ $\mathrm{ml}$ 1-Bromophenol blue, $5 \mathrm{mM}$ DTT). Five ul of each sample were loaded on a protein SDSpage gel and proteins resolved by electrophoresis. After semi-dry blotting of proteins onto PVDF membrane, filters were screened using anti-GFP rabbit polyclonal antibody (1: 2000 dilution) followed by anti-IgG rabbit polyclonal antibodies (Thermofisher, 1: 1000 dilution) to detect and determine TSWV reporter gene expression levels.

\section{Results}

\section{Viral $\mathbf{N}$ proteins from plant- and animal-infecting members of the Bunyavirales localize at cytoplasmic processing bodies and stress granules}

To analyse whether a co-localization with cytoplasmic RNA processing $(\mathrm{P})$ bodies is generic to the $\mathrm{N}$ protein from members of the order Bunyavirales, $\mathrm{N}$ proteins from several plantand animal infecting segmented (-)ssRNA viruses were fused with GFP and transiently coexpressed with the cytoplasmic PB marker protein DCP1a fused to RFP in plant leaves and animal cells respectively. In situ analysis showed that a portion of $\mathrm{N}$ proteins from animalinfecting SBV, SNV and CCHFV colocalized with the PB marker DCP1a-RFP in Vero cells (Fig. 4.1A, white arrow). In planta, similarly, the $\mathrm{N}$ proteins from plant-infecting counterparts TSWV and RSV were observed to co-localize with PB marker DCP1a-RFP (Fig. 4.1A, white arrow). Earlier studies on the TSWV structural $\mathrm{N}$ and glycoproteins showed that these 
proteins exhibited similar trafficking and localization behaviour in animal cells, compared to their structural homologs from animal infecting bunyaviruses (Kikkert et al. 2001; Snippe et al. 2005; Snippe et al. 2007). Therefore, TSWV N was also expressed in animal cells and its localization relative to $P$ bodies analysed. The results showed that in animal cells, like in plant cells, TSWV N protein co-localized with PB (Fig. 4.1A, white arrow). Although earlier studies showed a colocalization of SNV and TSWV N with P bodies, during our studies from all viruses analysed, irrespective of plant or animal-infecting viruses, the $\mathrm{N}$ protein did not completely co-localize with PB upon transient expression. Repeated analyses revealed that a part of the $\mathrm{N}$ protein consistently appeared in close proximity to $\mathrm{PB}$. To test whether these signals localized to SG, the cytoplasmic RNA granules that mostly appear adjacent PB, the $\mathrm{N}$ proteins from SBV, SNV, CCHFV and TSWV were co-expressed with the SG marker G3BP1 in Vero cells. All of the tested N proteins showed partial colocalization with SGs (Fig. 4.1B, white arrow). In plant cells, TSWV and RSV N proteins were transiently co-expressed with the SG marker G3BP1-RFP and also showed a colocalization with the SG marker protein to some extent. Similar with the observations made on the PB marker, the TSWV N protein colocalized with the SG marker in both animal and plant cells.

Besides the colocalization of N protein to either PB or SG (Fig. 4.1A, white and yellow arrows), the $\mathrm{N}$ protein randomly dispersed throughout the cell (Fig. 4.1A, purple arrow). To determine its spatial distribution into more detail, the localization of $\mathrm{N}$ relative to the observed $\mathrm{PB}$ and SG was quantified (Fig. 4.1C and D). To this end, and to allow statistical analyses, at least 10 cells were collected for each sample/treatment. The number (and size) of the granules was quantified using ImageJ Analyse Particle Analyser (for details see M\&M). From all N proteins analysed, the CCHFV N protein showed the highest degree of co-localization to the observed PB (64\%) upon its expression in Vero cells, while in 7\% of the observed PB the fluorescence signals of the N protein localized neighbouring the PB (Fig. 4.1C). A co-localization of SNV N and $\mathrm{PB}$ was observed in only $2 \%$ of the observed $\mathrm{PB}$, whereas $24 \%$ of the fluorescence signals showed up close to PB. For the SBV N protein, $29 \%$ of the observed PB showed a co-localizing $\mathrm{N}$ and $12 \%$ showed a neighbouring localization. Upon expression of the RSV N protein in $N$. benthamiana plant cells a colocalization with PB was observed in $16 \%$ of the cases, whereas $13 \%$ localized in close proximity. When TSWV N protein was transiently expressed in Vero cells and $N$. benthamiana cells, the $N$ protein co-localized to PB in $48 \%$ and $43 \%$ of the observed $\mathrm{PB}$, respectively. A similar variation was observed when the $\mathrm{N}$ proteins of SBV, SNV, CCHFV, TSWV and RSV were expressed in Vero cells and/or plant cells, respectively, and the localization was quantified relative to the observed SG (Fig. 4.1D). SBV N showed a high 
association (78\%) with SG in animal cells while RSV N showed a high association (81\%) with SG in plant cells. TSWV N protein also colocalized to a high number of SGs in both animal and plant cells. Although the numbers vary in between all viruses, the data altogether indicate that, in contrast to earlier (limited) reports suggesting a co-localization of $\mathrm{N}$ with PB only, the $\mathrm{N}$ protein from a wide range of viruses of the bunyavirales co-localize with both PB and SG.
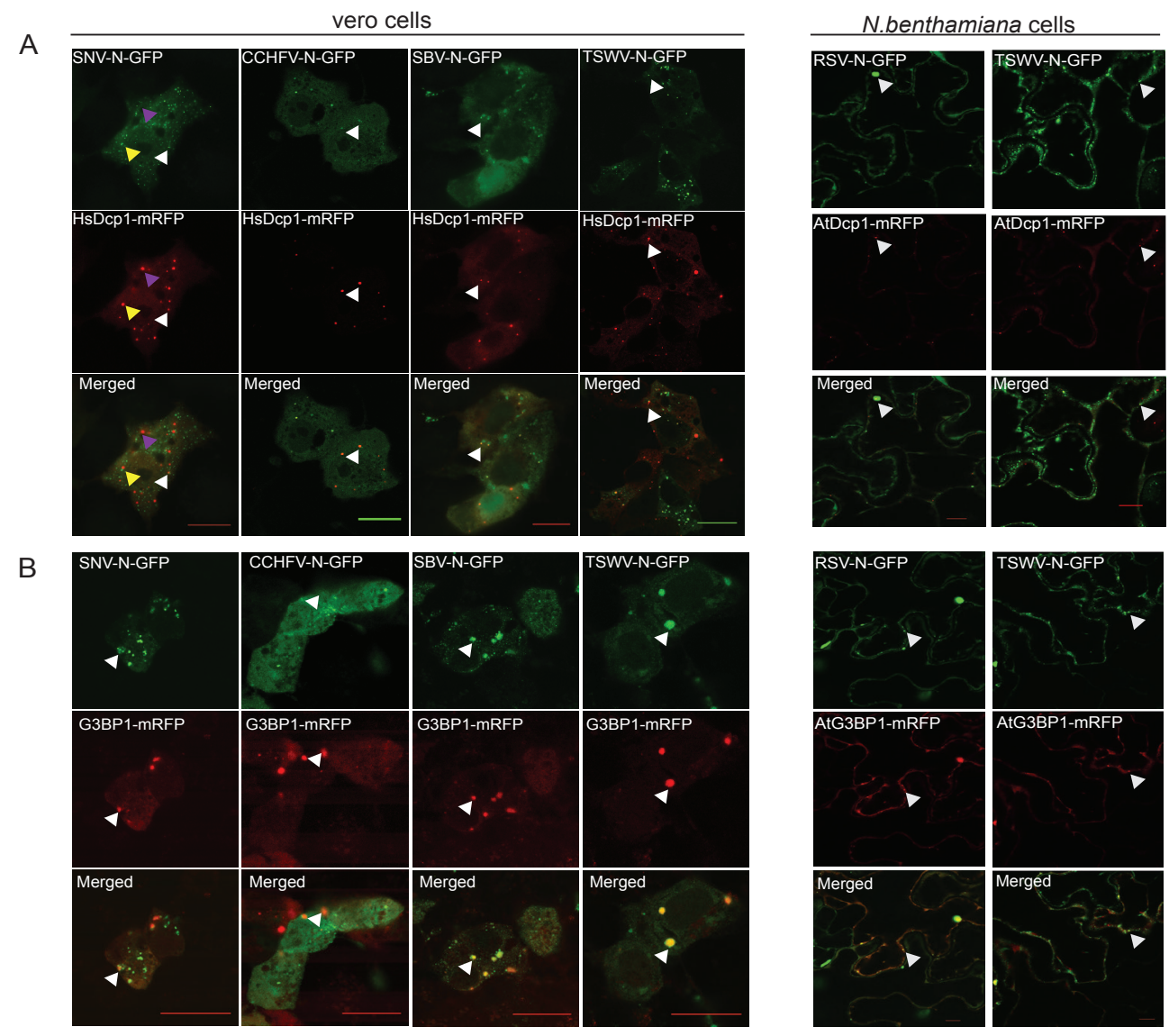

Figure 4.1 Localization and quantification of different NSV N proteins relative to PB/SG in plant cells and animal cells. (A) Confocal microscopy observation of SNV, CCHFV, SBV and TSWV N-GFP protein localization relative to P body marker protein HsDCP1-mRFP (left panel) in Vero cells, RSV and TSWV N-GFP protein localization relative to P body marker protein AtDCP1-mRFP in plant cells (right panel). Bar is 10uM. (B) Confocal microscopy observation of SNV, CCHFV, SBV and TSWV N-GFP protein localization relative to SG marker protein HsG3BP1-mRFP (left panel), RSV and TSWV N-GFP protein localization relative to SG marker protein AtG3BP1-mRFP in plant cells. Scale bar is $10 u M$. 
C
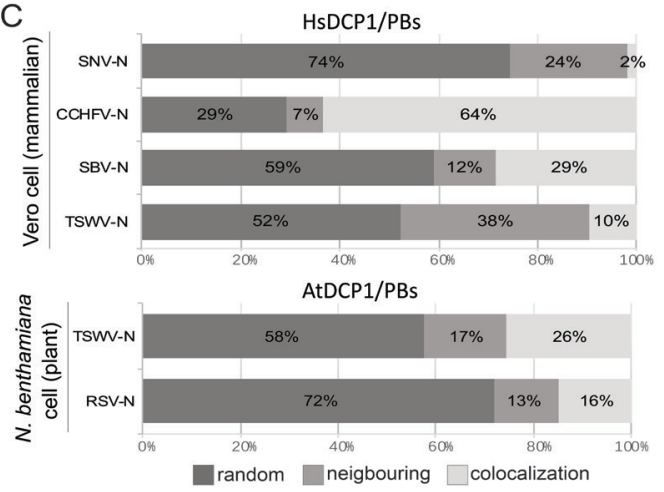
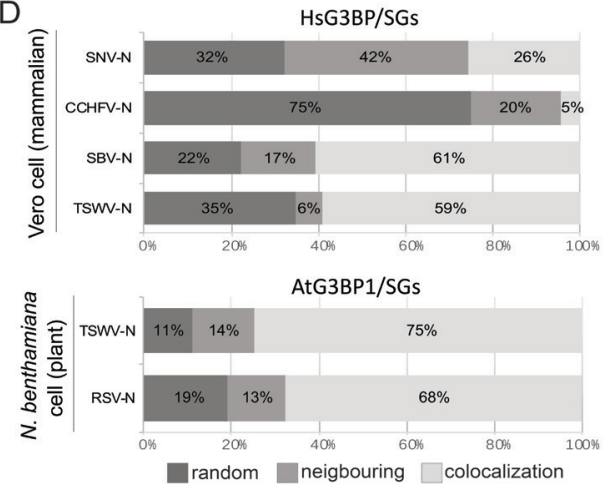

Figure 4.1 Localization and quantification of different NSV N proteins relative to PB/SG in plant cells and animal cells. (C) Quantification of $\mathrm{P}$ body localization relative to different $\mathrm{N}$ proteins. Colocalization, neighbouring and random localization ratio were quantified for SNV, CCHFV, SBV and TSWV in animal cells and TSWV, RSV in plant cells. (D) Quantification of SG localization relative to different N proteins. Colocalization, neighbouring and random localization ratio were quantified for SNV, CCHFV, SBV and TSWV in animal cells and TSWV, RSV in plant cells.

\section{$\mathrm{N}$ protein $\mathrm{P}$ body/SG association during viral infection}

To test whether the spatial distribution of transiently expressed $\mathrm{N}$ proteins relative to both PB and SG also occurred during a normal infection, the experiments were repeated but now in the presence of virus. To this end, TSWV N-GFP and SBV N-GFP were transiently expressed in the additional presence of the PB marker in plant respectively animal cells that were infected with the corresponding viruses prior to the experiment. Thirty hours post transfection with SBV N-GFP and the PB marker, animal cells were infected with SBV. After 24 hrs cells were analysed and showed a strong association of SBV N with P bodies (Fig. 4.2A). Transient expression following TSWV infection was conducted on the infected leaf 5 -days post mechanical inoculation. Also here, the results showed that a strong association of P bodies with TSWV N (Fig. 4.2B).

In analogy, transiently expressed N-GFP was localized relative to SGs during a viral infection. In animal cells, the infection with SBV induced formation of SGs (Fig. 4.2C) and a colocalization of $\mathrm{N}$ protein with the SG marker was observed (Fig. 4.2C). In planta, TSWV N protein and SG marker were co-expressed in infected leaf tissue 5-days post mechanically inoculation. However, no SG formation was observed in TSWV infected leaf tissue (Supplementary Fig. 4.1). To stimulate the induction of SGs, plants were subjected to heat stress, after which TSWV N colocalized in most cases of the SG observed (Fig. 4.2D). 

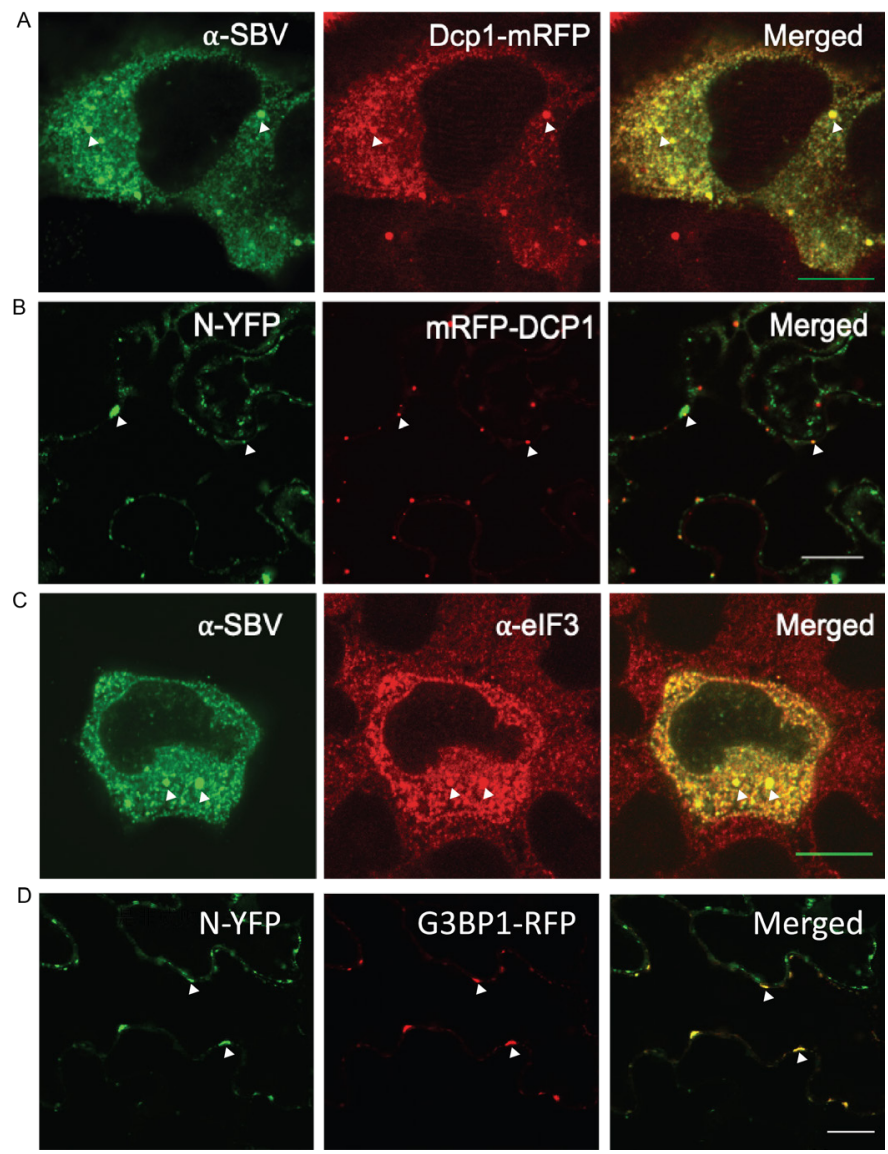

Figure 4.2 Localization of TSWV-N and SBV-N relative to PB/SG under viral infection. (A) HsDCP1-mRFP construct was first transfected to the cells and followed with SBV infection, immunostaining was performed for mRFP and SBV. Scale bar is 10uM. (B) $5 \mathrm{~d}$ post TSWV infection, TSWV-N-GFP and AtRFP-DCP1 were agro-infiltrated to infected leaves, confocal microscopy was observed at $48 \mathrm{hpi}$. Scale bar is 10uM. (C) HsG3BP-mRFP construct was first transfected to the cells and followed with SBV infection, immunostaining was performed for mRFP and SBV. Scale bar is 10uM. (D) 5d post TSWV infection, TSWV-N-GFP and AtRFP-G3BP1 were agro-infiltrated to infected leaves, confocal microscopy was observed at $48 \mathrm{hpi}$. Scale bar is $10 \mathrm{uM}$.

\section{TSWV N protein preferably localizes to stress granules docked on $\mathrm{P}$ bodies, and the formation of SG docked on PB promoted by viral infection}

Since all $\mathrm{N}$ proteins analysed in this study strongly associated to both cytoplasmic RNA granules when expressed transiently, as well as during a viral infection (for TSWV and SBV), another experiment was performed in which TSWV and SBV N proteins were co-expressed with PB and SG markers simultaneously. In agreement with earlier results, when TSWV 
$\mathrm{N}$ protein was transiently expressed it localized at SG and at $\mathrm{PB}$, but interestingly also colocalized with SG docked on PB, in both animal cells and plant cells (Fig. 4.3A). In order to quantify the spatial distribution of TSWV $N$ to any of these foci/condensates, PB and SG were first classified into three localization patterns and next the number of cases of TSWV $\mathrm{N}$ co-localizing to these stages was counted (Fig. 4.3B). The three defined localization profiles of PB versus SG (Fig. 4.3B) were: individual distribution (I), docking (co-localizing) with SG (D), and neighbouring (close) to SG (C). Upon quantification a large number of the docking stage (D) was associated with TSWV N protein, whereas (I) and (C) patterns were less enriched in $\mathrm{N}$ protein. In Vero cells, $94 \%$ of the docking stage (D) revealed the presence of TSWV N protein and $73 \%$ of $C$ pattern was associated with the $N$ protein (Fig. 4.3B). In N. benthamiana plant cells, $100 \%$ of the PB-SG docking complexes and $98 \%$ of closely distributed PB and SG showed the presence of TSWV N protein (Fig. 4.3B). When SBV N protein was transiently expressed in animal cells, $100 \%$ of the docking stages (D) observed showed the presence of SBV N protein, whereas $75 \%$ of the neighbouring (C) pattern exhibited an association with $\mathrm{N}$ protein (Fig. 4.3B).

Considering that viral infections are known to influence the occurrence and spatial distribution of cytoplasmic PBs and SGs, experiments were performed to analyse whether viral infections of TSWV and SBV also affected the occurrence and number of SG-PB docking stages. To this end, the numbers of individual PBs and SGs, as well as PB-SG docking stages were counted during either SBV-infection in animal cells or TSWV infection of plant tissues (Fig. 4.3C). In addition, PBs and SGs were also analysed under non-viral stress conditions for comparison (Fig. 4.3C). In Vero cells, 40\% PB docked to SG during arsenite treatment, whereas upon a challenge with SBV, 56\% of PB docked to SG (Fig. 4.3C left panel). Meanwhile, the rate of PB neighbouring to SG increased from $11 \%$ to $17 \%$. In $N$. benthamiana plant cells, PB docked to SG with a high rate after both heat shock treatment and TSWV infection. When cells were only treated with heat shock, $56 \%$ of PB docked to SG. Upon TSWV infection, 63\% of PB docked to SG (Fig. 4.3C, right panel). Altogether these data point towards an altering and dynamic distribution profile of $\mathrm{N}$ protein relative to the cytoplasmic PBs and SGs, that is not restricted to PB in contrast to earlier reports, and in which a viral infection clearly affects PB and SG dynamics. 

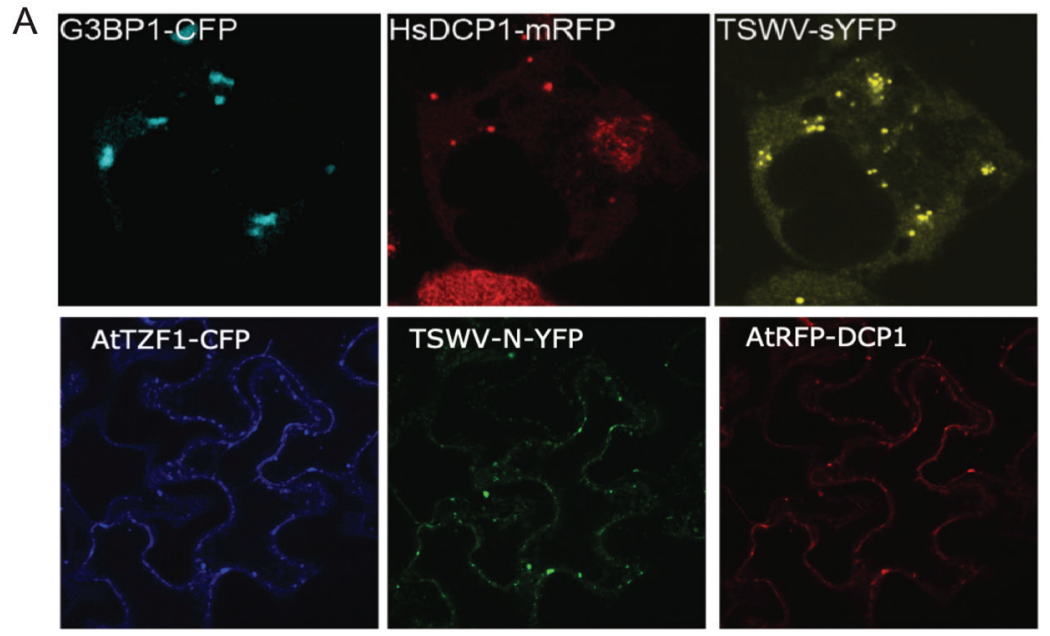

B

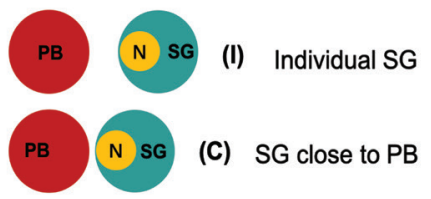

N SG (D) SG docked with PB

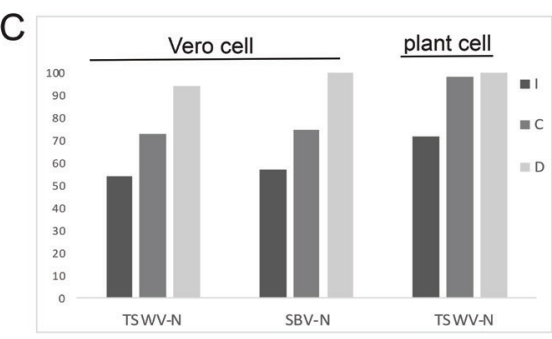

D

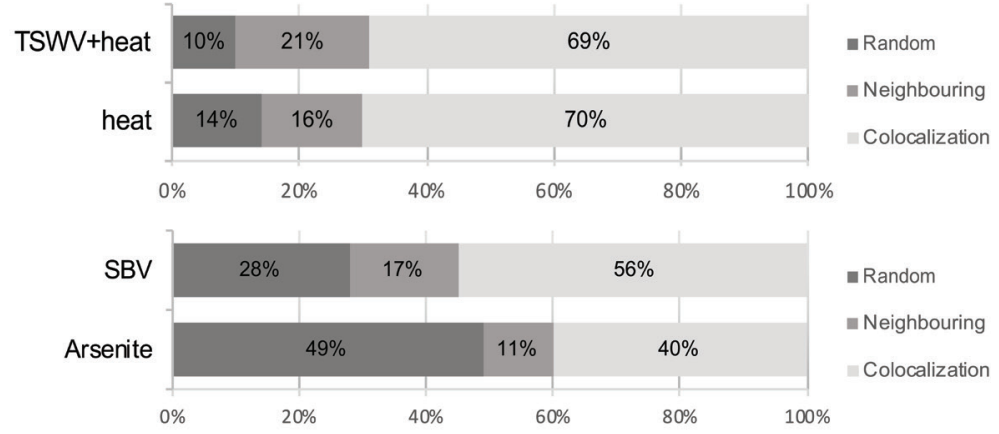

Figure 4.3. Localization and quantification of $\mathbf{N}$ proteins with both $\mathrm{PB}$ and $\mathrm{SG}$ marker proteins and $\mathrm{PB} / \mathrm{SG}$ association. (A) Transient expression of SBV N protein in Vero cells and conservation of the spatial distribution of N protein with PB and SG markers (Upper panel). Transient expression of TSWV N protein in N.benthamiana cells and observation of the spatial distribution of N protein with PB and SG markers (lower panel). (B) Graphic description of different patterns of $\mathrm{N}$ protein associated with SG linked with PB (Left panel). The indication "I" represents individual SG which shows random localization with PB, " $\mathrm{C}$ " represents SG close to PB. "D" represents SG docked with PB. (C) Quantification of N protein associated with different types of SG. (D) Quantification of PB and SG association types under heat and TSWV/heat conditions in plant cells (left panel) and quantification of PB and SG association types under arsenite and SBV infection in animal cells (right panel). 


\section{Silencing of different P body-related genes differentially influences TSWV replication rates}

Considering the clear link to PB and SG, their role as putative sources for capped RNA leader sequences to prime viral genome transcription was hypothesized. To test this idea, Tobacco rattle virus (TRV) induced gene silencing (VIGS) was performed to first knock down the expression of PB elements and subsequently analyse the effect on viral replication. For PB knock-down, DCP5 and UPF1 were selected as two candidate genes. The DCP5 gene encodes a $P B$ component that coordinates RNA de-capping activity with core de-capping enzymes (Xu and Chua 2009a). UPF1 is a regulator of the nonsense-mediated decay (NMD) pathway and facilitates the transport of nonsense RNA to $P$ bodies for subsequent degradation (Chicois et al. 2018; Kim and Maquat 2019). When plants were silenced on either DCP5 or UPF1, they showed a normal phenotype like those from GUS-silenced plants (Fig. 4.4A).

A
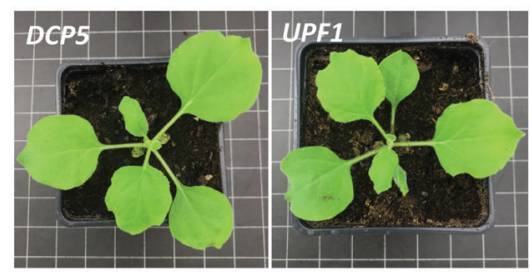

C
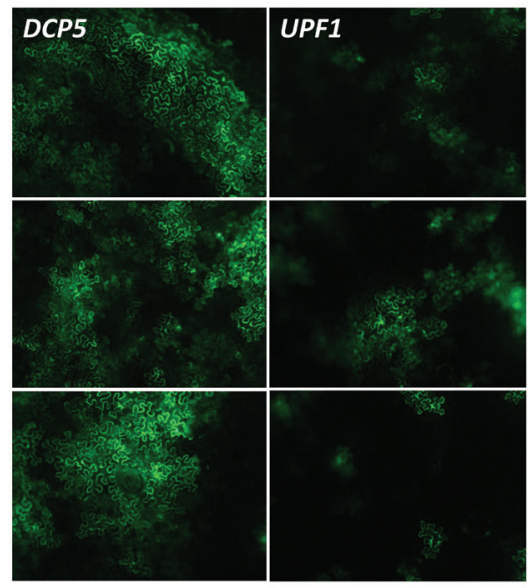

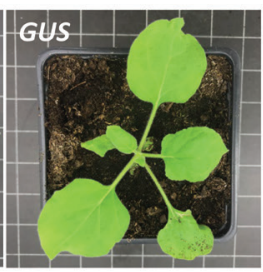

B
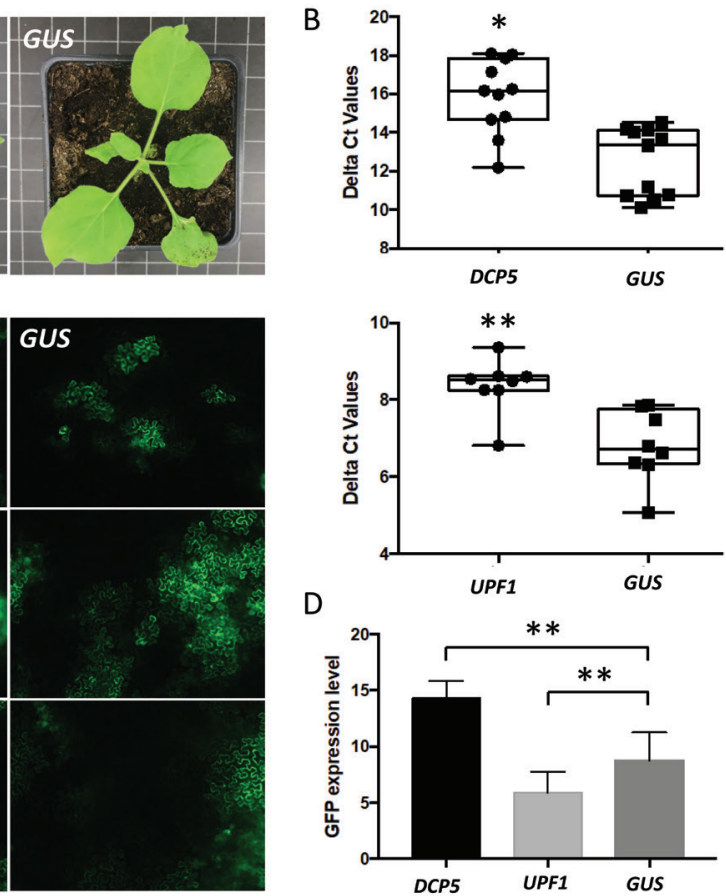

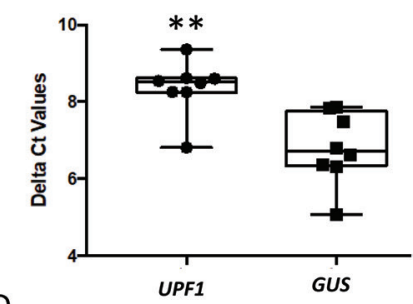

D

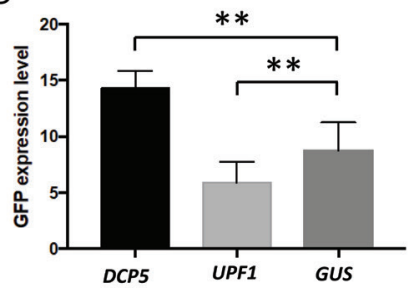

Figure 4.4 The effect of silencing $P$ body elements on viral replication. (A) Plant phenotype after silencing DCP5 and UPF1, compared to GUS silenced control plant. (B) qRT-PCR assay to quantify the silencing efficiency. ${ }^{*} p$-value $<0.05, * *$ p-value $<0.01, * * *$ p-value $<0.001$. (C) Green fluorescence resulting from TSWV replication in plants silenced on $\mathrm{P}$ body elements, and control plants. Plants were infiltrated TSWV-eGFP 2.5-week post silencing and harvested at around $60 \mathrm{hpi}$. (D) Statistical analysis of GFP intensity in the silenced plants and control plants. 
When those silenced plants were additionally checked on PB formation after transient expression of AtDCP1-mRFP, neither of the silenced genes seemed to affect condensation of AtDCP1-mRFP into PB (-like) aggregates (supplementary Fig. 4.2). When leaf samples were collected and tested by qRT-PCR on DCP5/UPF1 transcriptional expression levels, the delta $\mathrm{Ct}$ value indicated that both genes were indeed successfully silenced (Fig. 4.4B). Three-week post VIGS, plants silenced on DCP5/UPF1 were infiltrated with TSWV L, M and S-eGFP genome constructs to rescue TSWV and determine the rate of viral replication by GFP fluorescence levels (Feng et al. 2020). Whereas in UPF1-silenced plants, TSWV-GFP expression levels were only slightly less than those collected from GUS-silenced plants (Fig. 4.4C), in DCP5-silenced plants the GFP expression level was higher than in GUS-silenced plants (Fig. 4.4C). Quantification was performed to show the significance of GFP signals in several silenced plants (Fig. 4.4D).

In analogy to the knock down of PB elements, TRV-induced gene silencing was also performed on SG components and their effect on TSWV infection analysed. To this end, several genes were selected. G3BP-like gene and $R b p 47$ gene were selected for their function in the assembly of SGs (Protter and Parker 2016). Elf4A and elf4E genes were selected due to the dynamic localization to SGs (Protter and Parker 2016). When plants were silenced on G3BP-like and Rbp47 the plant phenotype remained normal (Fig. 4.5A). A qRT-PCR on total RNA purified from silenced leaf samples showed a clear and significant knock down of both genes (Fig. 4.5B). When leaves of plants silenced on G3BP-like and Rbp47 were subsequently infiltrated with constructs for transient expression of TZF1-CFP, the formation of SG (under heat stress) was clearly affected in comparison to GUS-silenced plants (supplementary Fig. 4.3). Upon subsequent infiltration of these leaves with TSWV L, M and S-eGFP genome constructs to rescue TSWV and determine the rate of viral replication by GFP fluorescence levels, the expression level of GFP in both G3BP-like and Rbp47 silenced plants dramatically increased compared to GUS-silenced plants (Fig. 4.5C and D).

To test the role of SG in SBV replication, a G3BP knock-out cell line was used and infected with SBV. After $24 \mathrm{hrs}$ p.i. SBV titres were determined and, in comparison to wild type (unsilenced) cells, these were higher in G3BP knock-out cells (Fig. 4.5E), and in agreement with the observations made on TSWV in plant cells. 
A
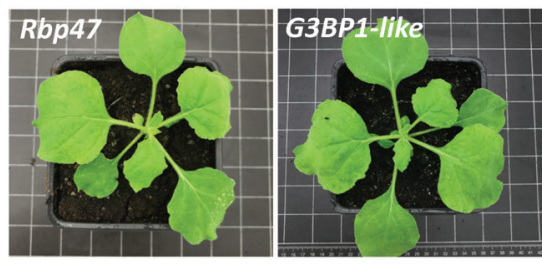

C
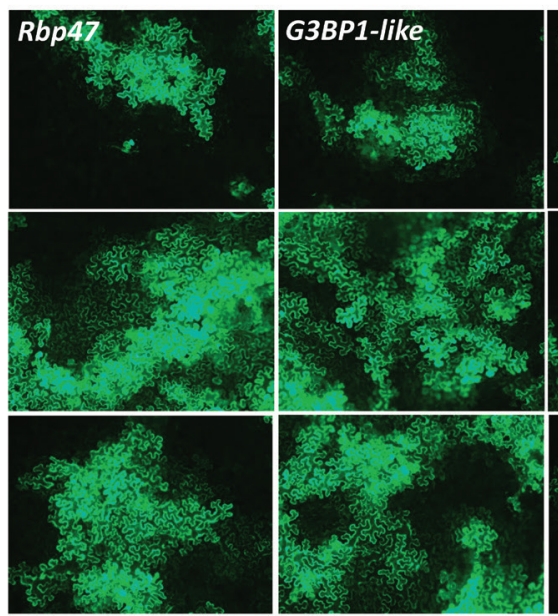
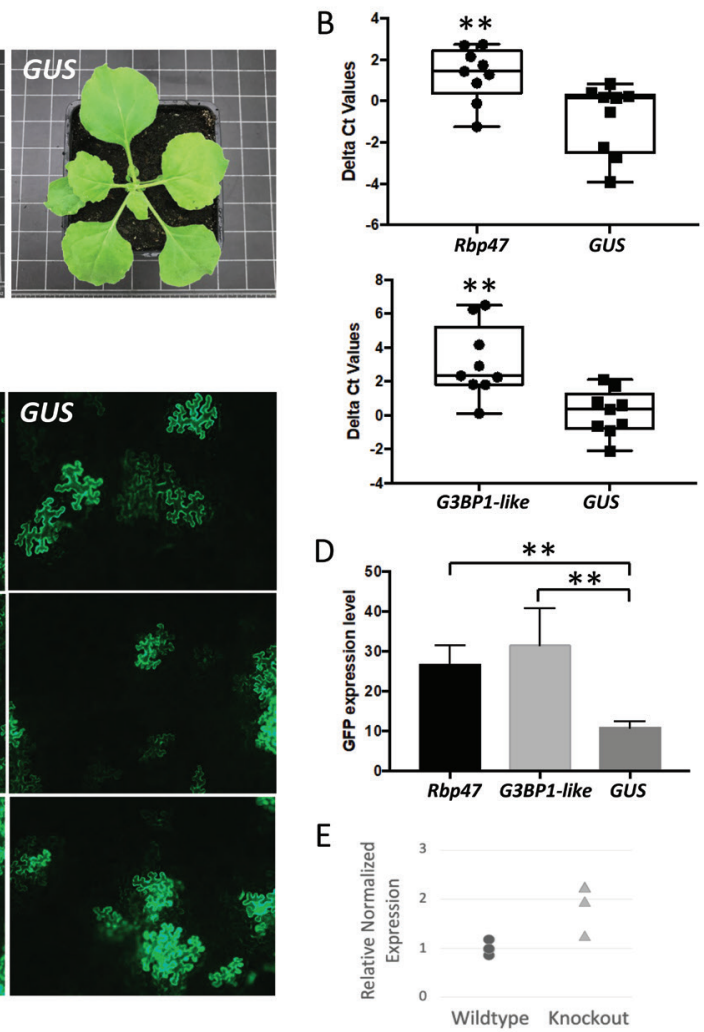

Figure 4.5 The effect of silencing SG assembly components on viral replication. (A) Plant phenotype after silencing Rbp47 and G3BP1-like, compared to GUS silenced control plant. (B) qRT-PCR assay to quantify the silencing efficiency. ${ }^{*} p$-value $<0.05, * * p$-value $<0.01, * * * p$-value $<0.001$. (C) Green fluorescence resulting from TSWV replication in plants silenced on SG assembly components, and control plants. Plants were infiltrated with TSWVeGFP 2.5-week post silencing and harvested at around $60 \mathrm{hpi}$. (D) Statistical analysis of GFP intensity in the silenced plants and control plants. (E) qRT-PCR assay to check viral accumulation in mammalian cells.

elF4A and elF4E are translation initiation factors that enter SGs for storage upon stress induction and are again released to engage in translation of the mRNA when the stress is relieved (Protter and Parker 2016). Similar to G3BP-like and Rbp47, plants were silenced on these two genes to test the effect on TSWV infection. When the phenotype of silenced plants was checked no difference was observed between elF4E silenced plants and GUSsilenced control plants (Fig. 4.6A). However, plants silenced on elF4A exhibited stunting and leaf deformation (Fig. 4.6A). A qRT-PCR showed that both genes were effectively silenced (Fig. 4.6B). When those plants were subsequently challenged with a TSWV infection, induced by infiltration of the infectious TSWV L, M and S-eGFP genome constructs, GFP expression 
level was hardly observed in eIF4A-silenced plants compared to GUS-silenced control plants. However, and interestingly, elF4E-silenced plants showed an enormously higher GFP expression, indicating high replication levels of the virus (Fig. 4.6C and D).
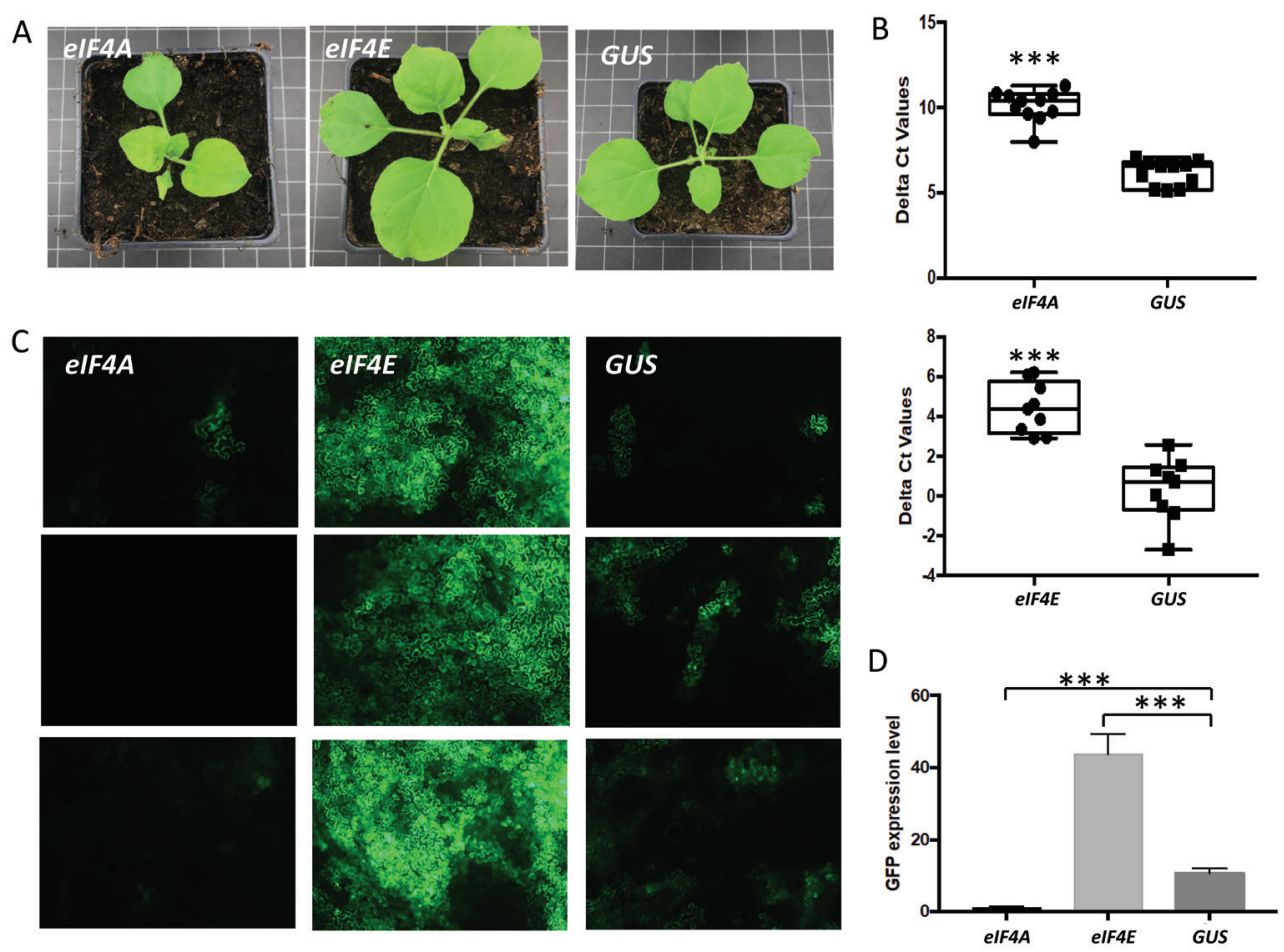

Figure 4.6 The effect of silencing other SG components on viral replication. (A) Plant phenotype after silencing eIF4A and elF4E, compared to GUS silenced control plant. (B) qRT-PCR assay to quantify the silencing efficiency. $*$ p-value $<0.05, * *$ p-value $<0.01, * * *$ p-value $<0.001$. (C) Green fluorescence resulting from TSWV replication in plants silenced on SG components, and control plants. Plants were infiltrated with TSWV-eGFP 2.5-week post silencing and harvested at around $60 \mathrm{hpi}$. (D) Statistical analysis of GFP intensity in the silenced plants and control plants.

\section{Simultaneous silencing of PB and SG components still allows for high viral replication levels}

Considering that the individual silencing of components from PBs or SGs differentially affected viral replication rates, and mostly did not show a decline in viral replication, raised the question whether PB and SG would only play a redundant role as a source for capped-RNA leaders, and allowing the virus to alternate between both granules for viral cap-snatching. Silencing of one would allow for an escape and promote usage of the other. To test this 
hypothesis, N. benthamiana plants were silenced on DCP5 and G3BP1-like simultaneously. Plants did not exhibit a changed phenotype compared to GUS-silenced control plants (Fig. 4.7A), and upon qRT-PCR analyses of transcript levels plants were selected that showed silencing of both genes (Fig. 4.7B). When these plants were subsequently infiltrated with TSWV L, M and S-eGFP genome constructs and the rate of viral replication analysed based on the amount of GFP fluorescence, the level of GFP expression in silenced plants was higher than in GUS-silenced control plants (Fig. 4.7C and D). A similar experiment is currently being prepared to determine SBV titres in G3BP knock-out Hela cells that additionally have been silenced on DCP1a. The results so far, at least shown for the plant infecting bunyavirus TSWV, indicated that a knock-out of elements from both PB and SG does not negatively affect viral replication rates, but rather points toward an antiviral role.
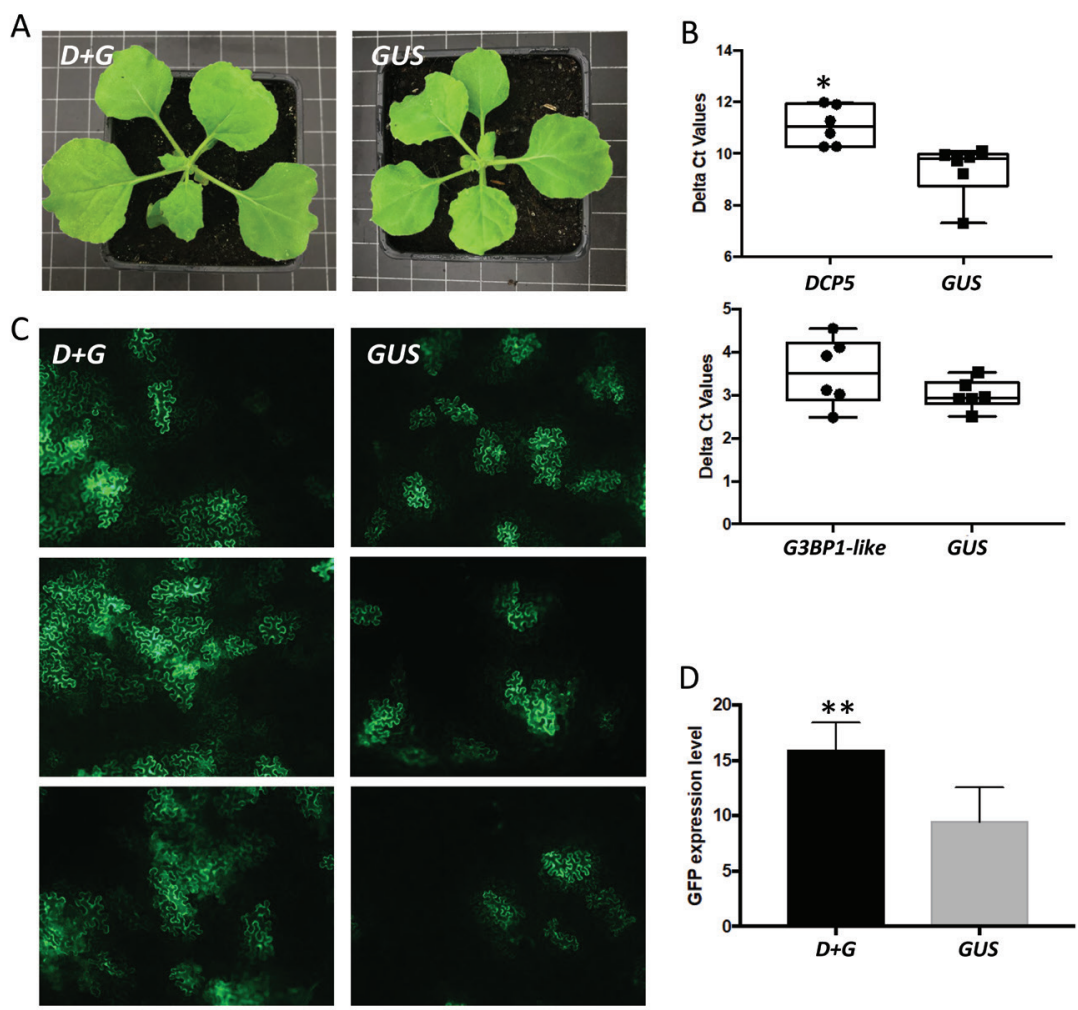

Figure 4.7 The effect of a simultaneous silencing of DCP5 and G3BP1-like on viral replication. (A) Plant phenotype after silencing DCP5 and G3BP1-like, compared to GUS silenced control plant. (B) qRT-PCR assay to quantify the silencing efficiency. ${ }^{*} \mathrm{p}$-value $<0.05,{ }^{*} \mathrm{p}$-value $<0.01, * * * \mathrm{p}$-value $<0.001$. (C) Green fluorescence resulting from TSWV replication in plants silenced on DCP5 and G3BP1-like, and GUS silenced control plants. Plants were infiltrated with TSWV-eGFP 2.5-week post silencing and harvested at around $60 \mathrm{hpi}$. (D) Statistical analysis of GFP intensity in the silenced plants and control plants. 


\section{$\mathbf{N}$ proteins localize to the perinuclear region and silencing of nucleocytoplasmic transport RanGAP factors negatively affects TSWV replication}

Since the concomitant silencing of $\mathrm{P}$ bodies and SGs only led to a slight increase in viral replication, and not to an expected cumulative effect (combined from the silencing of $\mathrm{PB}$ and SG individually), we speculated on other cytoplasmic foci (upstream of the mRNA trafficking routes leading to SG and $\mathrm{PB}$ ) that could present potential sites/sources for the virus from where to snatch capped-RNA leader sequences. Considering that the silencing of elF4E, not only a component of SG but also an essential factor for a nuclear mRNA export pathway, led to a major increase in viral replication (Fig. 4.6B), we speculated on the nuclear pore complex (NPC) as a potential target/foci for cap-snatching. As the gate of nucleocytoplasmic transport, NPCs serve for continuous mRNA export to the cytoplasm and thereby offer a constant efflux/supply of capped RNA. Prior to testing this, the localization of N proteins relative to RanGAP, an important player of the nucleocytoplasmic transport machinery at Nuclear pore complexes (NPCs), was analysed. Upon in planta co-expression of TSWV N-GFP and RSV N-GFP with RanGAP-mcherry, both N proteins, besides the observations described above in relation to $\mathrm{PB}$ and $\mathrm{SG}$, also revealed a perinuclear localization that overlapped with RanGAP (Fig. 4.8A). When the experiment was repeated, but now in the presence of the RanGAP unique N-terminal domain (WPP domain) fused to a nuclear localization signal (NLS), part of the $\mathrm{N}$ protein showed up in the nucleus with RanGAP as well, indicating an interaction of the $\mathrm{N}$ protein with the nucleocytoplasmic transport machinery of which RanGAP is a component (Fig. 4.8B). Next, RanGAP genes were silenced to inhibit the export and release of nuclear mRNA into the cytosol. Whereas silencing of RanGAP2 resulted in some slight stunting (Fig. 4.8B), silencing of both RanGAPs showed a slightly more severe phenotype. A quantitative RT-PCR was performed to test the silencing efficiency and revealed a significant reduction of the mRNA transcript level (Fig. 4.8C). Subsequently, TSWV L, M and S-eGFP genome constructs were infiltrated to rescue TSWV (containing a S-RNA encoding GFP gene) and determine the rate of viral replication by GFP fluorescence levels. The expression of GFP on single RanGAP2 gene silenced plants was similar as GUSsilenced plants. However, the expression of GFP on double RanGAPs silenced plants was clearly lower than in GUS-silenced plants. 


\section{Chapter 4}
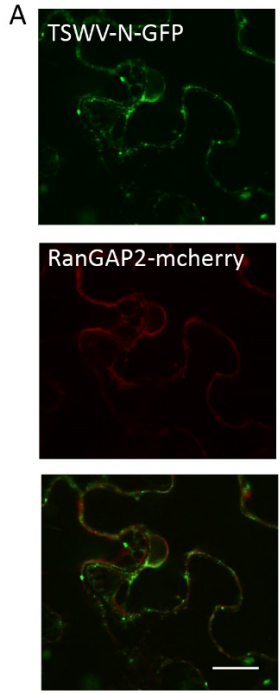

C

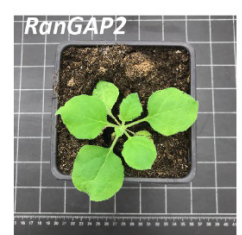

E

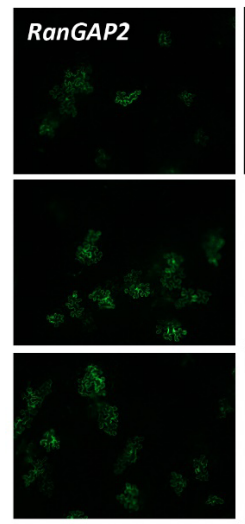

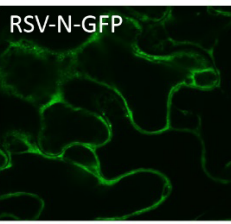

RanGAP2-mcherry
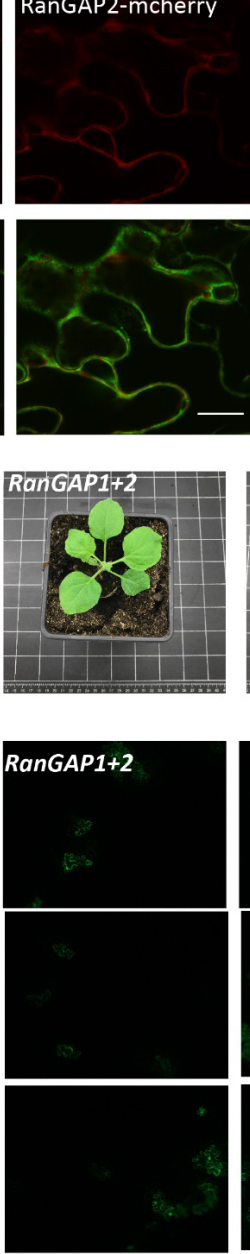
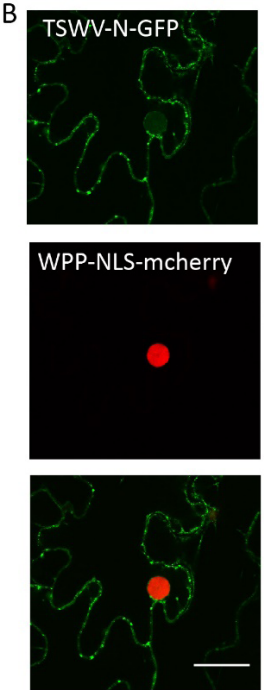

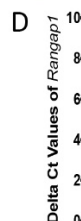
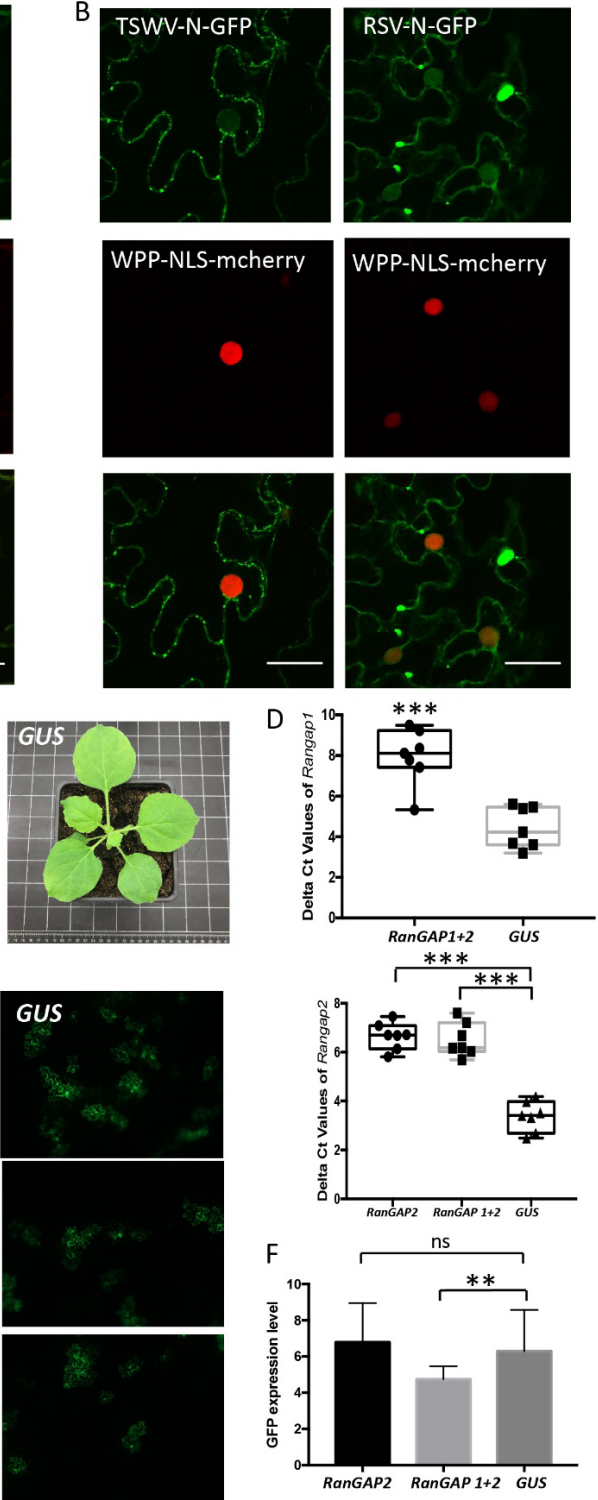

Figure 4.8 Co-localization analysis of $\mathbf{N}$ with AtRanGAP2 and effect of RanGAP silencing on viral replication. (A) In situ localization of RSV and TSWV N-GFP protein relative to RanGAP2-mcherry in plant cells. Bar presents 10uM. (B) In situ localization of RSV and TSWV N-GFP protein relative to WPP-NLS-mcherry in plant cells. Bar presents 10uM. (C) Plant phenotype after silencing RanGAPs, compared to GUS silenced control plant. (D) qRT-PCR assay to quantify the silencing efficiency. ${ }^{*} p$-value $<0.05,{ }^{* *} p$-value $<0.01,{ }^{* * *} p$-value $<0.001$. (E) Green fluorescence

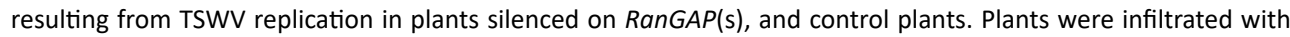
TSWV-eGFP 2.5-week post silencing and harvested at around 60hpi. (F) Statistical analysis of GFP intensity in the silenced plants and control plants. 


\section{Discussion}

P bodies are currently postulated/considered as the source for cap donors for cytoplasmicreplicating NSVs, needed to initiate viral genome transcription (Hopkins et al. 2013; Mir et al. 2008b). In this study, the relation of several segmented NSVs to PBs and other cytoplasmic granules/foci was further investigated. Here, it is shown that $\mathrm{N}$ proteins of different segmented, cytoplasmic-replicating NSVs either transiently expressed or during viral infection, not only localize at PBs but also at SGs (Fig. 4.1) (Malinowska et al. 2016; Reineke and Lloyd 2013b; Weber et al. 2008), and preferably at PB-SG docking stages. Silencing of PB and/or SG in plants resulted in differential effects on TSWV titres but mostly led to higher titres. TSWV/RSV N proteins furthermore revealed a peri-nuclear localization that overlapped with RanGAP, an important player of the nucleocytoplasmic (RNA) transport pathway through NPCs. Silencing of RanGAPs led to clearly reduced titres of TSWV. These results altogether indicated a possible redundancy of PB and SG in cap-snatching and a putative role of sources more upstream in the mRNA trafficking pathway, whose inhibition is more detrimental to viral genome transcription-replication initiation.

First data pointing towards PB as source of host cellular mRNAs for cap-snatching were collected from studies performed with the animal-infecting SNV hantavirus. Those studies revealed the usage of capped-RNA leaders from transcripts destined (by the NMDpathway) to PB for viral genome transcription, and a co-localization of the SNV N protein with PB (Mir et al. 2008b). Moreover, the SBV N protein exhibited affinity to cap analogue (Mir et al. 2010), which might explain its localization at foci enriched for mRNA, like PB. Recently, similar indications have been reported for TSWV N, and the TSWV N protein observed to (partially) localize to $P$ bodies as well. However, the usage of capped-RNA leaders from host cellular transcripts destined to PB for TSWV viral genome transcription was not quantified relative to the usage of functional transcripts. Also, no other putative sources enriched for host cellular mRNAs were pointed out, nor further investigated whether or not to play a role in the cap-snatching process besides $\mathrm{P}$ bodies, even though SNV was observed to surprisingly still replicate to similar levels in P body deficient cells (Mir et al. 2010). The latter findings indicated that the virus likely is able to use other sites/ cytoplasmic granules to support viral genome transcription, as also raised by the authors of the study on SNV. Whether these sites are additional or simply just upstream PB, the final destination of any capped-RNA molecule, still remained elusive. The findings from this study indicates at least some redundancy and/or usage of other sources upstream the 
cytoplasmic mRNA trafficking pathway. From a rational and deductive point of view, the observed co-localization of $\mathrm{N}$ protein to SG is not totally unexpected for several reasons. Firstly, although these granules are only transient and often arise as a result and (a)biotic stress responses, like viral infections, they contain functional mRNAs that are only stalled in translation. SGs thus provide in perfect substrate for cap-snatching, in contrast to PB, where mRNAs are de-capped and degraded. Secondly, with SGs often localizing in close proximity of $\mathrm{PB}$, and even known to exchange components during the formation of docking stages, a co-localization of $\mathrm{N}$ proteins to $\mathrm{PB}$ tempts to speculate and investigate a co-localization with SG as well. Our results, not only with SNV, for which earlier only a co-localization with PB was reported, but also with several other completely distinct (plant- and animal infecting) members of the Bunyavirales, showing a co-localization of the $\mathrm{N}$ protein with PB and SG, strengthened the observations made and support the idea of all these cytoplasmic replicating NSVs performing cap-snatching at more foci enriched for host cellular mRNAs, and not limited to PB only.

Previous studies have shown that PB and SG are different granules, but spatially, compositionally, and functionally they are tightly linked (Kedersha et al. 2005). The formation of PB-SG docking complexes allows for a specific mRNA exchange. Messenger RNAs destined for decay disassemble from polysomes and are first sorted at SG before they subsequently transport into PB (Kedersha et al. 2005). Considering that viral infections often lead to SG formation, viral infections may also boost the RNA interchange between PB and SG. The access of virus to capped RNA from both structures thus would be a very effective way to support viral genome transcription. The observation on both SBV and TSWV N proteins to preferably localize at docked PB/SG supports this point of view.

By taking out DCP5, a enzymatic coactivator of the de-capping process in PB (Xu and Chua 2009a), more capped-RNA molecules will remain available for cap-snatching. As expected, TSWV replication becomes up-regulated, as observed in this study, but also observed in another recent study on TSWV and earlier already reported on RVFV that DCP2 silencing in insect cells increased viral titres (Hopkins et al. 2013). By taking out UPF1, which interferes at the nonsense mediated RNA transportation to PB, TSWV replication is only slightly attenuated. Similar result has been shown in Arabidopsis UPF1 mutant which reduced the TSWV infection(Ma et al. 2019). The slight attenuation in viral replication indicates that NMD RNA-trafficking to PB is probably not the only source of cap donor molecular for TSWV. Notably, a recent study has pointed that RNA degradation of molecules entering the NMD 
pathway seems to occur in "polysomal-derived" complexes, and not at PB (Sulkowska et al. 2020). This further indicates the importance of capped RNAs from sources other than/ besides PB, e.g. upstream into the cascade of the translational machinery.

When G3bp-like or Rbp47, both components from SGs, another putative cap donor source, were taken out the viral titter turned much higher. In other words, breaking SG assembly promoted viral replication and indicated antiviral activity of SGs, as already observed and reported with many viruses (Chapter 2). However, and alternatively, SGs may also act redundantly with PB. When the virus accesses PB more efficiently and silencing of SGs shifts some of its stalled mRNA to PB, viral genome transcription would benefit and titres go up. However, SGs are not always present in the cell, not even and always during viral infections, but when they are formed during biotic/abiotic stress conditions and translatable mRNAs are stalled/stored, they present an additional/alternative escape for cytoplasmic replicating segmented NSVs to support cap-snatching. It thus makes sense that when cells are inhibited in SG formation, these viruses still maintain their ability to replicate well. To further confirm if SGs indeed facilitate cap-snatching, experiments are needed to analyse the usage of cap leader sequences that originate from transcripts transported to SGs. Furthermore, it remains to be investigated whether the silencing of SG formation does lead to a shift of the stalled mRNAs to PBs.

In case PB and SG present the sole source for capped-RNA leaders to support cap-snatching, a combined silencing of DCP5 (leading to increased levels of capped RNA in PB) and G3BP (leading to a possible shift of stalled mRNA to PB) would lead to a major increase in viral transcription-replication levels. Unexpectedly, and surprising, only a slight increase of TSWV replication was observed, and not up to a higher level than when these granules were taken out individually. This raises the question on the use of additional/alternative sources/sites from where to take capped RNA, more upstream of the RNA trafficking pathway leading to PB/SG. Earlier studies on SNV already showed that even when cells were depleted from PB the virus was still able to effectively replicate (Mir et al. 2010). In our previous study, we also found the colocalization of SBV N protein with (the cytoplasmic precursor stages of) $U$ bodies, but this was relatively minor. When summarizing the observations on PB and SG, it remains to be noted that these condensates not only reflect end points of enriched RNAs but are also relatively easy to discern. This might blur our views and lead to ignoring possible upstream sources of first-hand capped RNA molecules for viral cap-snatching. 
The localization of TSWV and RSV N protein in the perinuclear region, partly overlapping with RanGAP, an important player of the nucleocytoplasmic transport machinery at the NPC, pointed towards the idea of accessing mRNA during nucleocytoplasmic transport through nuclear pores. As the gate of nucleocytoplasmic transport, NPCs serve for continuous mRNA export to the cytoplasm and thereby offer a constant efflux/supply of capped RNA. Intriguingly, TSWV N localization altered by a RanGAP domain fused with nuclear localization signal, and suggests an interaction between $\mathrm{N}$ and RanGAP. Silencing of both RanGAP (1 and 2) genes (reduction by $30 \%$ ) affected TSWV replication, and indicates that inhibition of the nuclear-cytoplasmic mRNA trafficking pathway is quite detrimental to viral replication. This is most likely caused by a reduction in the pool of mRNAs at the cytoplasmic side of NCP, resulting from a reduced RanGAP-mediated mRNA nuclear efflux. This idea agrees with data obtained on eIF4E silencing, where an increase in TSWV replication was observed. Knowing that elF4E is also involved in nuclear export of specific mRNAs, in specific on the pick-up of mRNAs from the nuclear efflux at NCP for further release in the cytoplasm, the hypothesis on nuclear pore as first-hand source has become quite appealing. However, this idea only holds true when elF4E silencing indeed leads to an accumulation of capped RNA accumulation at the (cytoplasmic side of) NCP. Considering that elF4E is also involved in other process, like protecting mRNA from degradation and initiating translation, as well as resident in SGs, more studies are needed to support this idea.

As we have described in chapter 2, cellular RNA granules present a complicated network. In previous studies, Mir et al have shown that transcripts ending up in $\mathrm{P}$ bodies are used for SNV cap-snatching. Although SNV prefers transcripts with PTCS that are assumed to move towards PB, viral transcription-replication was not affected when cells were depleted from $P$ bodies. The authors did not further investigate this and only suggested that the virus might use transcripts from other sources. Recent studies also linked P bodies to TSWV cap-snatching, but also these studies were limited, since the observed increase of TSWV replication in DCP2-deficient Arabidopsis upon heat stress was not further investigated. This study shows that the $\mathrm{N}$ protein of several cytoplasmic replicating, segmented NSVs not only co-localize at PB but also are found at SGs, and silencing of elements from these granules, as well as from the upstream cytoplasmic mRNA trafficking pathway, indicates that these viruses may likely use capped-RNA leader sequences from granules/sources not limited to $\mathrm{PB}$ only. The questions on how and to what extent/ratio these viruses use cap donors from PB and/or SG, relative to the entire pool of cytoplasmic mRNAs, or whether the NPC present the first-hand and major site for cap-donor supply, remain a challenge for the near future. 


\section{Acknowledgements}

We thank dr. Peter Moffett (Department of Biology at the Université de Sherbrooke in Sherbrooke, Québec, Canada) for providing the TRV RNA2 constructs for silencing of DCP2, DCP5, dr. N. Kedersha for providing mammalian RNA granules markers Hs decapping 1(HsDCP1), HsCaprin-1 and HsG3BP1, dr. A. Maizel for providing plant P body marker RFPDCP1, and dr. Björn Krenz was for providing tandem zinc finger protein 1 fused with RFP (TZF1-RFP) and G3BP1-RFP, dr. Aska Govers (Wageningen University) for providing TRV2 constructs for silencing RanGAPs and AtRanGAPs constructs for localization observstion, Prof. dr. Monique van Oers (Wageningen University) is acknowledged for critically reading the manuscript prior to submission. The authors acknowledge N. de Ruijter from the Wageningen Light Microscopy Centre and Ying Zheng from public experiment platform of college of plant protection, Nanjing Agricultural University for assistance in cellular imaging. This research was financed partially by China Scholarship Council. The author also kindly thank Henk Smid from uniform of Wageningen University for the support of N.benthamiana plants. 


\section{Supplemental data}

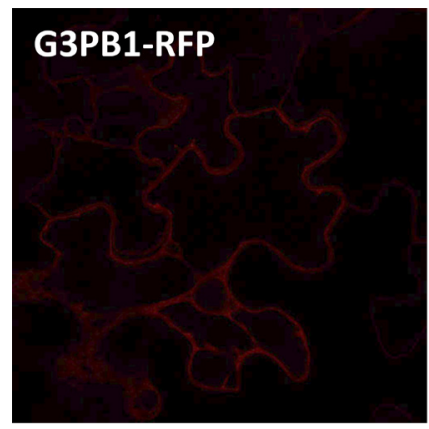

Supplemental Figure 4.1 The expression of G3BP1-RFP at normal condition.
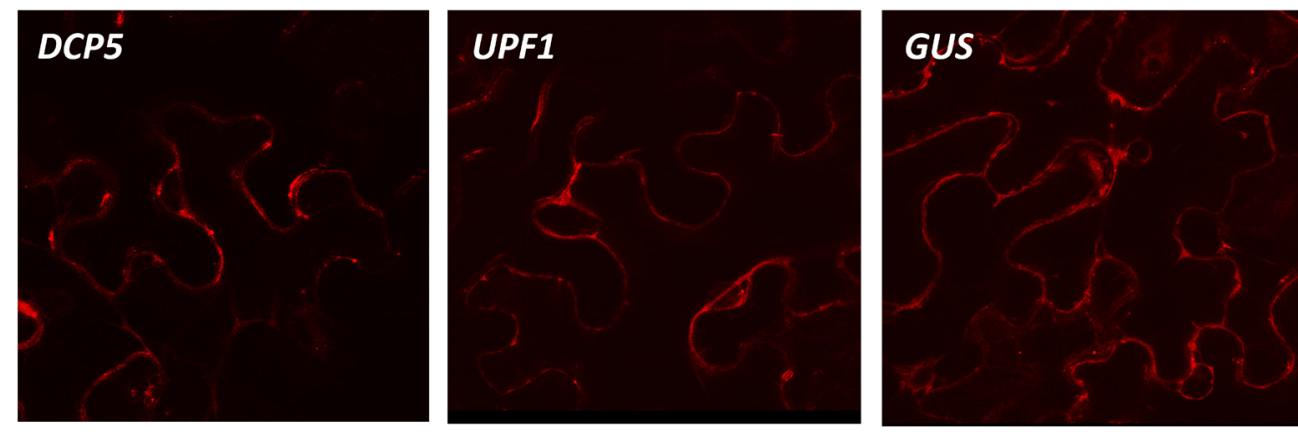

Supplemental Figure 4.2 The expression of DCP1-RFP on different silenced plants at heat stress condition.
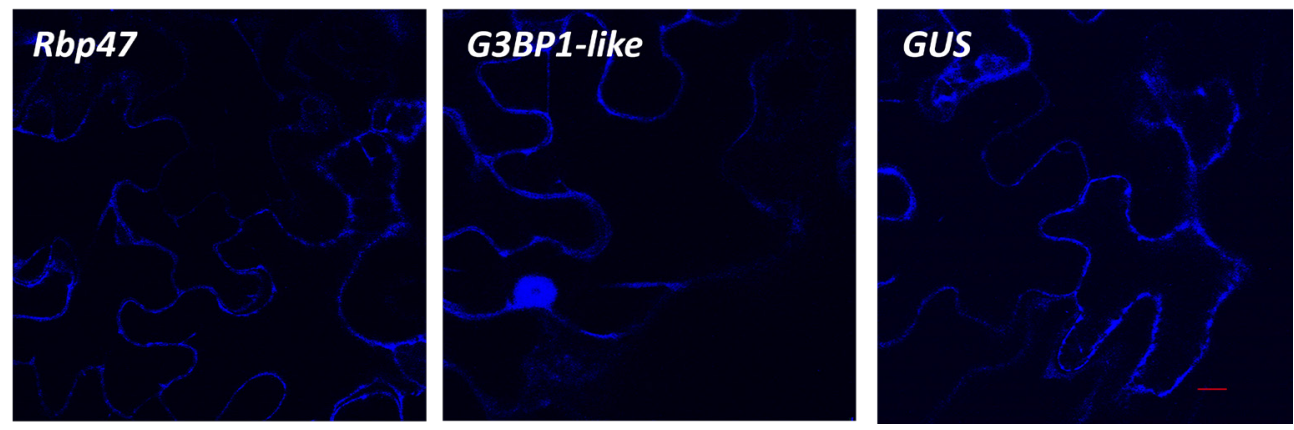

Supplemental Figure 4.3 The expression of G3BP1-CFP on different silenced plants at heat stress condition. 
Supplemental Table 4.1 Primers used in this chapter.

\begin{tabular}{|c|c|}
\hline Name & Sequence \\
\hline F_Sacll_eGFP & AACCGCGGATGGTGAGCAAGGGCGAG \\
\hline R_Sacll_eGFP & AACCGCGGTTACTTGTACAGCTCGTC \\
\hline F_Sacll_mRFP & ACCGCGGATGGCCTCCTCCGAGGAC \\
\hline R_Sacll_mRFP_stop & ACCGCGGTTAGGCGCCGGTGGA \\
\hline F_attB1_N_TSWV & GGGGACAAGTTTGTACAAAAAAGCAGGCTTAACCATGTCTAAGGTTAAGCTCACT \\
\hline R_attB2_N_TSWV & GGGGACCACTTTGTACAAGAAAGCTGGGTAAGCAAGTTCTGCGAGTTT \\
\hline F_attB1_N_SBV & GGGGACAAGTTTGTACAAAAAAGCAGGCTTAACCATGTCAAGCCAATTCATTTTT \\
\hline R_attB2_N_SBV & GGGGACCACTTTGTACAAGAAAGCTGGGTAGATGTTGATACCGAATTG \\
\hline F_attB1_N_SNV & GGGGACAAGTTTGTACAAAAAAGCAGGCTTAACCATGAGCACCCTCAAAGAAGTG \\
\hline R_attB2_N_SNV & GGGGACCACTTTGTACAAGAAAGCTGGGTAAAGTTTAAGTGGTTCTTG \\
\hline F_attB1_adapt & GGGGACAAGTTTGTACAAAAAAGCAGGC \\
\hline R_attB2_adapt & GGGGACCACTTTGTACAAGAAAGCTGGGT \\
\hline F_attB1_CCHFV_N & CAAAAAAGCAGGCTCCACCATGGAAAACAAAATCGAAG \\
\hline R_attB2_CCHFV_N & CAAGAAAGCTGGGTAGCGCCAACATCATCT \\
\hline F_attb1_G3BP1 & GGGGACAAGTTTGTACAAAAAAGCAGGCTCCACCATGGTGATGGAGAAGC \\
\hline R_attb2_G3BP1 & GGGGACCACTTTGTACAAGAAAGCTGGGTACTGCCGTGGCGCAAGC \\
\hline F_attB1_caprin1 & GGGGACAAGTTTGTACAAAAAAGCAGGCTCCACCATGCCCTCGGCCACC \\
\hline R_attB1_caprin1 & GGGGACCACTTTGTACAAGAAAGCTGGGTAATTCACTTGCTGAGTGTTCAT \\
\hline F_attB1_DCP1A & GGGGACAAGTTTGTACAAAAAAGCAGGCTCCACCATGGAGGCGCTGAGT \\
\hline R_attB2_DCP1A & GGGGACCACTTTGTACAAGAAAGCTGGGTATAGGTTGTGGTTGTCTTTG \\
\hline F_q_PCR_G3BP & AGTAGAGGAACCTGAAGAAAGACAGC \\
\hline R_q_PCR_G3BP & CCATGTCATTACTGACAACTGCCTG \\
\hline F_q_PCR_GAPDH & CACCAGGGCTGCTTTTAACTC \\
\hline R_q_PCR_GAPDH & GGAATTTGCCATGGGTGGAATC \\
\hline F_q_PCR_SBV & TCAGATTGTCATGCCCCTTGC \\
\hline R_q_PCR_SBV & TTCGGCCCCAGGTGCAAATC \\
\hline F_DCP1a_qPCR & TATCACCAGCATCGCAGACC \\
\hline R_DCP1a_qPCR & TGGTAAGGGGAAGCTGACCT \\
\hline F_attB1_N_EMARaV & GGGGACAAGTTTGTACAAAAAAGCTGAACAGATGCCTATTATTCCAAAGCC \\
\hline R_attB2_N_EMARaV & GGGGACCACTTTGTACAAGAAAGCTGAACTTGTGGTTGTTTTGAAGCTTTC \\
\hline F_attB1_N_RSV & GGGGACAAGTTTGTACAAAAAAGCTGAACAGATGGGTACCAACAAGCCAG \\
\hline R_attB2_N_RSV & GGGGACCACTTTGTACAAGAAAGCTGAACTCTAGTCATCTGCACCTTCTGC \\
\hline F_RNAi_Nb-elF4E-like & GGGGACAAGTTTGTACAAAAAAGCTGAACAGGGAAAGTGGACAATGAGCTTTTC \\
\hline R_RNAi_Nb-elF4E-like & GGGGACCACTTTGTACAAGAAAGCTGAACTCTACGCAGAATAACGATTCTTGG \\
\hline F_BgllI_AteiF4E & GAAGATCTATGGCGGTAGAAGACACTCC \\
\hline
\end{tabular}




\begin{tabular}{|c|c|}
\hline R_BglII_AteiF4E & GAAGATCTTCAAGCGGTGTAAGCGTTC \\
\hline F_BgllI_AtTZF1 & GAAGATCTATGATCGGCGAAAATAAAAACC \\
\hline R_BglII_AtTZF1 & GAAGATCTACCGAGTGAGTTCTCTCTACTGAG \\
\hline F_RNAi_Nb-DCP5 & GGGGACAAGTTTGTACAAAAAAGCTGAACAGAATCTGTCAAGATCATCTCTC \\
\hline R_RNAi_Nb-DCP5 & GGGGACCACTTTGTACAAGAAAGCTGAACTTCTGTGTGTGTGGCCTTTAC \\
\hline F_q_PCR_TSWV & GCTTCCCACCCTTTGATT \\
\hline R_q_PCR_TSWV & TCCCGAGGTCCTTGTATT \\
\hline F_q_PCR_elf4E-like & GTGAGGTGGAAGAGGAAGG \\
\hline R_q_PCR_elf4E-like & GGAGAAGGTGTAAATGGGTC \\
\hline F_q_PCR_DCP5 & AATAACGGTGTCTGCTCTG \\
\hline R_q_PCR_DCP5 & CAGTTTGCGAGATTGTGG \\
\hline F_q_PCR_actin & TCCTGATGGGCAAGTGATTAC \\
\hline R_q_PCR_actin & TTGTATGTGGTCTCGTGGATTC \\
\hline F_q_PCR_EF1a & AGCTTTACCTCCCAAGTCATC \\
\hline R_q_PCR_EF1a & AGAACGCCTGTCAATCTTGG \\
\hline RBP47_qPCR_F & GGTCTCCGTGAAAATACCTGC \\
\hline RBP47_qPCR_R & GAAAGACGAACTGCCTGCTT \\
\hline RBP47_RNAi_F & GGGGACAAGTTTGTACAAAAAAGCAGGCTTATAGCACTGGTGAAAAGCGTG \\
\hline RBP47_RNAi_R & GGGGACCACTTTGTACAAGAAAGCTGGGTATTGTTGCTGTGCTGATGGTT \\
\hline G3BP_RNAi_F & GGGGACAAGTTTGTACAAAAAAGCAGGCTTAATGATGCAGTCCAGGTTCCA \\
\hline G3BP_RNAi_R & GGGGACCACTTTGTACAAGAAAGCTGGGTACTAGAAGGTGCTGGAGGAGG \\
\hline G3BP_qPCR_F & TGTGGTCGAGGAGAAGAGGT \\
\hline G3BP_qPCR_R & TAACCCCTGCCACCTCCATA \\
\hline Nbelf4a-RNAi-F & GGGGACAAGTTTGTACAAAAAAGCAGGCTTACAACTTTCTGCTCTGGAGT \\
\hline Nbelf4a-RNAi-R & GGGGACCACTTTGTACAAGAAAGCTGGGTATCCTTGAAACCTCTTGAGAGC \\
\hline UPF1_RNAi-F & GGGGACAAGTTTGTACAAAAAAGCAGGCTTAATTGCGTTATTCAGGGGATGC \\
\hline UPF1_RNAi-R & GGGGACCACTTTGTACAAGAAAGCTGGGTACCAAAACGGCGAGGAAGTGTA \\
\hline
\end{tabular}




\section{Chapter 5}

\section{High throughput sequencing of snatched host cellular mRNA leader sequences for viral genome transcription initiation and identification of the host donor genes}

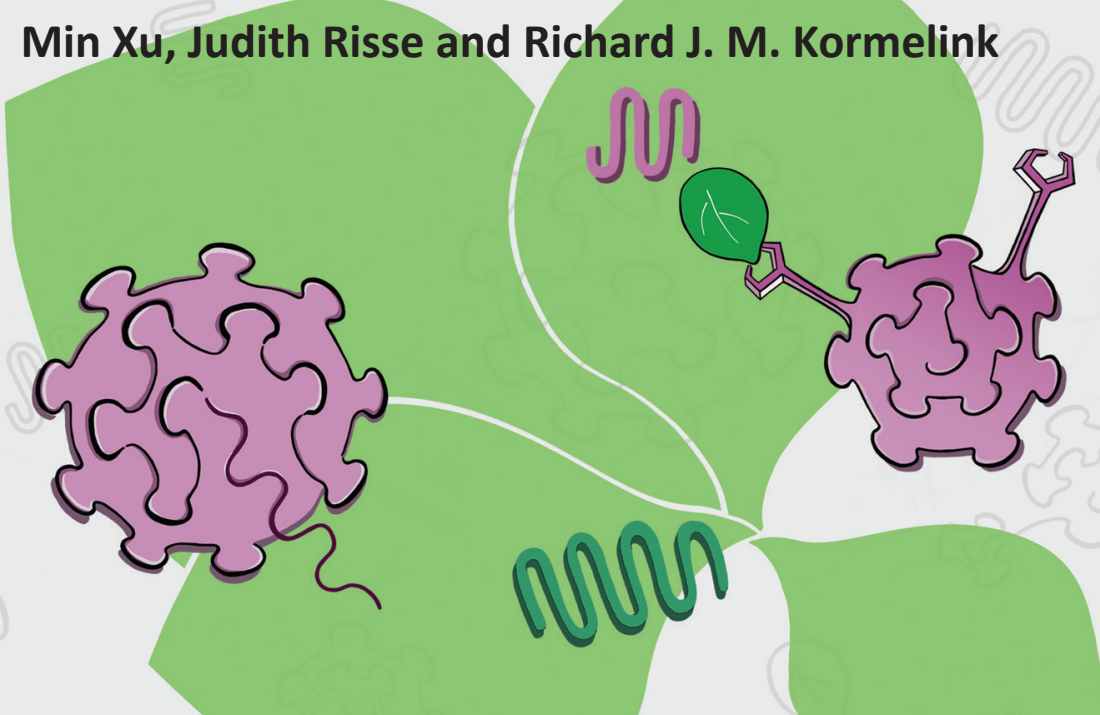

This chapter will be submitted in a slightly modified version as:

Min Xu, Judith Risse and Richard Kormelink (2021). High throughput sequencing of snatched host cellular mRNA leader sequences for viral genome transcription initiation and identification of the host donor genes. 


\section{Abstract}

Cap-snatching is a mechanism applied by segmented, negative strand (-) RNA viruses (NSVs) to initiate genome transcription. So far, the cap donor source of cytoplasmicreplicating NSVs has remained elusive. Recently, N protein localization studies pointed to the nucleopore and cytoplasmic RNA granules as potential source for providing capped RNAs but conclusive evidence is still lacking. Identifying the origins of donor transcripts, which could hint to possible sources, might help to solve this issue. Here, using Tomato spotted wilt virus (TSWV) as a representative, $5^{\prime}$ race RT-PCR was performed to clone the $5^{\prime}$ ends of TSWV N mRNAs for high throughput sequencing (HTS). Analysis of the nonviral heterogenous, host-derived leader sequences revealed that their sizes ( 10-20 nt), cleavage sites (at A or AG) and motif features matched with previous studies. Mapping the capped-leader sequences to the 5' UTR region of genes encoded by the Nicotiana tabacum genome, identified 348 donor genes and which were specifically enriched in cellular photosynthesis pathway genes. Nineteen of those were clearly expressed differentially at normal condition versus heat stress conditions. Furthermore, in vivo cap donor competition experiments showed that TSWV was able to use exogenous GFP, nsGFP (with premature stop codon (PTC)) and long GFP (LGFP) transcripts for cap-snatching, in which the usage of LGFP was promoted by heat treatment. These findings altogether indicate that during cap-snatching TSWV uses transcripts that not necessarily are restricted and destined to $P$ bodies as suggested by other studies. 


\section{Introduction}

In eukaryotic cells, pre-mRNA 5' capping is a critical process for mRNA maturation and protein translation(Ramanathan et al. 2016). By means of capping, capped mRNAs avoid being degraded and are exported to the cytosol, for most of the bulk mRNAs to become translated (Wilkie et al. 2003). The presence of the 5' cap leads to the recruitment of translation initiation factors and ribosomes, which will start scanning from $5^{\prime}$ end to initiate translation at the first (optimal) start codon (Lopez-Lastra et al. 2010). Viruses rely on the host translational machinery to produce viral proteins, translated from viral transcripts that mostly also contain a 5' cap (Raoult and Forterre 2008). Whereas many viruses are able to cap their own transcripts, the single strand negative-sense RNA viruses from the family Orthomyxoviridae, in the order Bunyavirirales, are not able to do this and instead adopted cap-snatching to solve this issue. During cap-snatching the viral transcriptase complex binds and subsequently cleaves short capped-RNA leader sequences from host cellular mRNAs, to next use these as primers on the viral genome to initiate transcription. As a result, viral mRNA molecules from these viruses are distinguished by the additional presence of a nonviral, non-templated leader sequence at their 5' ends.

Many studies have been conducted to investigate the mechanism of cap-snatching and Influenza virus is among the best studied viruses on this issue. Influenza viruses are nuclearreplicating and snatch capped-leader sequences in the nucleus. Its polymerase complex consists of three subunits, PA, PB1 and PB2, with each of them containing a specific role. Subunit PA contains the endonuclease activity that cleaves $5^{\prime}$ capped-leaders bound at the cap by a cap-binding domain (CBD) in PB2. PB1 contains the core domain of polymerase catalytic activity and is needed for elongation (Guilligay et al. 2008; Stevaert and Naesens 2016; Yuan et al. 2009). The entire complex binds, via PA, to the carboxy-terminal domain (CTD) of host RNA polymerase II (pol II) (Engelhardt et al. 2005). This enables influenza virus to directly access a pool of $5^{\prime}$ cap structures of nascent messenger transcripts. Once cleaved, the 5' capped-leaders, aligned on the viral RNA template by base pairing (GeertsDimitriadou et al. 2011a; Geerts-Dimitriadou et al. 2011b), are being elongated by PB1 to generate viral mRNAs.

Cap-snatching studies have also been conducted with several cytoplasmic-replicating segmented NSVs and have revealed some similar characteristics and subtle differences when compared to influenza virus. Although repetitive sequences were already reported 
in viral transcripts from several viruses, the prime and re-alignment (PAR) event has first been found and described with hantavirus and next observed and demonstrated to occur with Tomato spotted wilt virus (TSWV) (2001), Influenza virus (2011) and other (plant- and animal infecting) cytoplasmic-replicating segmented NSVs (Duijsings et al. 2001; Garcin et al. 1995; Yao et al. 2012). Follow-up studies on TSWV and Influenza revealed that these viruses exhibit a preference for capped-RNA leader molecules that are able to align on the $3^{\prime}$ viral genomic RNA by base-pairing, during which multiple base-paring increases the usage of capped leader sequences. The optimal size of capped leaders varies among different segmented NSVs, however is no longer than $20 \mathrm{nt}$ in general. For example, the size of capped leader sequences is in the range of 12-18 nt for TSWV, 11-14 for La Crosse virus, 12-20 nt for RSV and 9-13 for Influenza virus (Geerts-Dimitriadou et al. 2011a; Olschewski et al. 2020).

Whereas the source of cap donors has been identified for the nuclear replicating Influenza viruses, this has remained elusive for the cytoplasmic-replicating segmented NSVs. A few studies on Sin nombre hantavirus (SNV), Rift valley fever virus (RVFV) and TSWV, have pointed towards cytoplasmic $P$ bodies as a source for cap-snatching (Mir et al. 2008b) (Hopkins et al. 2013) (Ma et al. 2019), in which SNV appears to exhibit a preference for transcripts containing a premature translation termination codon (PTC). Affinity to $5^{\prime}$ cap structures, or a role in translational enhancement has also been reported for the $\mathrm{N}$ protein of several cytoplasmic replicating NSVs. However, some questions still remained unsolved, e.g. how to explain that the SNV virus replicates well when cells are depleted from $\mathrm{P}$ bodies (Cheng and Mir 2012). A recent study showed that $\mathrm{N}$ proteins from different (plant- and animal infecting) bunyaviruses not only associate with $\mathrm{P}$ bodies but stress granules (SGs) as well (chapter 4), and a colocalization has especially been observed with SGs-docked on PB. In tissues where PB elements are silenced, preventing decapping of mRNA, TSWV titres increase. Upon silencing of SG condensation factor G3BP, TSWV titres also increase, pointing to either antiviral activity of SG and/or a redundancy towards PB. Furthermore, the $\mathrm{N}$ proteins of TSWV and rice stripe virus (RSV) also localize to the nuclear envelope together with RanGAP2, a cofactor in nuclear export. Silencing of RanGAP homologs attenuates TSWV infection (Chapter 4). The results indicate that the cytoplasmic replicating NSVs might be able to target multiple sites enriched for host cellular mRNAs to support cap-snatching. Whether these viruses prefer one major site/source for cap-snatching, and if so, which one, still remains to be solved. 
In two studies on influenza virus, viral mRNAs were shown to be enriched in leader sequences derived from (pol II-generated) noncoding RNAs (ncRNAs) and small nuclear RNAs (sncRNAs) which could be distinguished by the presence of specific sequence features (Gu et al. 2015; Li et al. 2020). Here, we have taken a similar approach, and performed High throughput sequencing (HTS) to analyse a large collection of non-viral leader sequences of TSWV mRNAs for the presence of potential motifs or clues that could hint to the cytoplasmic source from where cytoplasmic replicating NSVs take capped leader sequences. In addition, exogenous (functional, non-functional and long) transcripts, and destined to different cytoplasmic granules/sites, have been offered to TSWV-infected plants in pair-wise competitions to test their usage as cap donor.

\section{Materials and methods}

\section{Plant material and virus inoculation}

Nicotiana benthamiana plants were kept in climate chambers at $23^{\circ} \mathrm{C}$ with a $8 \mathrm{~h}$ light/16 $\mathrm{h}$ dark cycle. Four-week-old plants were inoculated with inoculum from TSWV-infected $N$. benthamiana leaves ground in PB (0.01M Na2HPO4.H2O, 0.01M Na2H2PO4.2H2O, pH7.5) buffer. For heat stress, plants were incubated at $37^{\circ} \mathrm{C}$ for $3 \mathrm{hrs}$ prior to harvesting.

\section{pEAQ-GFP (modified), pEAQ-nsGFP and pEAQ-L-GFP construction}

GFP fragment was amplified and cloned into PEAQ-HT vector (Sainsbury et al. 2009). Site directed mutagenesis was performed within the UTR leader sequence at nucleotide position 12. Primers with mutagenesis were used to amplify the whole plasmid followed by Dpnl (NEB) enzymatic digestion to digest input plasmid DNA. The PCR fragment was purified, religated and recovered as plasmid after transformation into bacteria. The $P E A Q-G F P$ construct was modified to additionally introduce a premature stop codon at position nt10 of the GFP transcript leader sequence. To generate a long (L)-GFP construct, the GFP sequence was fused with a $2 A$ sequence and two tandem LacZ sequences into $P E A Q$ vector.

\section{Agrobacterium infiltration}

Constructs were transformed into Agrobacterium tumefaciens (GV3101). Cells harboring different constructs were grown overnight at room temperature and treated with infiltration buffer (10 mM MgCl2, $10 \mathrm{mM}$ MES, pH 5.9, and $150 \mu \mathrm{M}$ acetosyringone) for $3 \mathrm{~h}$ at room 
temperature. $N$. benthamiana leaves were infiltrated with combinations of suspensions containing a final optical density at $600 \mathrm{~nm}$ (OD600) of 0.5 per construct.

\section{RNA isolation, 5' RACE PCR and cloning}

Total RNA of systemically infected $N$. benthamiana leaves was extracted using Trizol Extraction Kit (invitrogen). Two ug total RNA was used for reverse transcription by Superscript II transcriptase (Thermo fisher). After reverse transcription, template-switch primer was added for further transcription (supplemental Table 1). Complementary DNA was amplified using template-switch primer and an internal primer tailed with an adaptor sequence (supplemental Table 1). Purified PCR products were used for 2 nd PCR amplicon sequencing (Eurofin Genomics, Germany). For cloning of leader sequences from GFP/nsGFP/ LGFP transcripts, primers without adaptors (supplemental Table 1) were used for the PCR amplification and products obtained were digested by $\mathrm{Ncol}$ and Pstl restriction enzymes (NEB). Digestion products were ligated into pGEM-T-Easy vector. Following transformation to DH10b cells, a colony PCR was performed to select positive colonies for further analysis. Sequence analysis of plasmids was performed by Eurofins (Germany).

\section{cDNA synthesis and Quantitative real-time PCR (qRT-PCR)}

Total RNA was extracted from the infiltrated leaf samples and used for first strand cDNA synthesis. To this end, 500ng total RNA was used per sample and reverse transcribed by M-MLV RT (Promega), using oligo-dT reverse primer following the manufacturer's protocol. Complementary DNA was diluted five times prior to further analyses. Specific primers for GFP and nsGFP were designed for quantitative analyses of RNA expression levels (see supplementary table 1). The qRT-PCR was performed in an ABI 7500 RealTime PCR system (Life Technologies). Actin-2 and EF1a served as internal controls to normalize the RNA levels of target gene expression between samples, using a relative quantification method.

\section{Annotation of 5' UTRs in Nicotina tabacco}

Nicotiana tabacum was chosen as reference genome as it is of better quality than $N$. benthamiana. Five_prime_UTR features were explicitly added to the genome annotation in a two-step approach. Genes without implicit 5' UTR (i.e. the coding sequence and first exon of the gene have the same starting coordinates) were extended with a hypothetical 5'UTR 
of 250 nt using a custom script (add5UTR.py). The implicit UTRs were then converted to the five_prime_UTR feature using the NCBI provided script add_utrs_to_gff.py (https://ftp.ncbi. nlm.nih.gov/genomes/TOOLS/).

For the alignment of putative host leader sequences to 5'UTR regions a masked Ntab reference genome was created using BEDTools (v 2.26.0, (Quinlan 2014)) mask where all bases outside of $5^{\prime}$ UTR annotations were converted to $\mathrm{N}$.

\section{Annotation of Ntab-TN90 genes with GO terms}

To test the snatched transcripts for overrepresentation of GO terms the Ntab-TN90 proteins were annotated using InterproScan (v 5.50-84.0, with -goterms (Cock et al. 2013)). GO terms were extracted from the gff3 output file, duplicates per gene removed and consolidated into a table for input into the R GO overrepresentation analysis (gotermsToAssoc.py).

\section{Detection of host capped leader sequence regions and target genes}

For all six sequenced samples, the 150PE reads were merged and primers removed using cutadapt (v1.11,(Martin 2011)) and vsearch merge (v2.1.1,(Rognes et al. 2016)). Merged reads combined with read 1 from the unmerged reads were aligned to a TSWV reference genome using minimap 2 (v 2.10-r764-dirty,(Li 2018) with default settings and sorted using samtools ( $v$ 1.11,(Danecek et al. 2021)). Using a custom script (capsnatch_merge. py) the softclipped section of each alignment to the TSWV reference was extracted and filtered to retain those between 10 and $100 \mathrm{nt}$ in length and with less than $80 \%$ AT content to remove polyA signals. The resulting sequences were written to a fastq output file. All extracted reads were reduced to unique reads using vsearch derep prefix and cluster fast (--id 0.9-min_length 10)and written to a separate file for input into Streme(Bailey 2020)

The softclipped fastq records were aligned to the masked Ntab-TN90 genome using bwa aln/ samse (bwa v 0.7.17-r1188, (Li and Durbin 2009)England) in combination with samtools sort. HTSeq-count (v 0.13.5,(Anders et al. 2015)) with feature five_prime_UTR was used to obtain counts per transcript. BEDTools genomecov was used to determine per base coverage of each alignment. 


\section{Identification of overrepresented motifs in host capped leader sequences}

The unique host capped leader sequences where tested for overrepresented motifs using Streme (Meme 5.3.2, with -maxw 18,(Bailey 2020)), once for the combined libraries of heat treated and control respectively and once for the mapped 5'UTR regions per treatment.

\section{Differentially snatched host capped leader sequences between heat- treated and control samples}

Counts files produced by htseq-count were analysed for differentially snatched transcripts using DESeq2 (v 1.26.0,(Love et al. 2014) in R ( $v$ 3.6.2) with the simple model treatment. Snatched transcripts were filtered only to retain those with at least 10 reads mapping to the five_prime_UTR feature in a minimum of three samples. Counts were normalized to library size using estimateSizeFactors. Euclidian distance between samples was calculated from rlog transformed normalized counts and plotted in a heatmap using pheatmap (v 1.0.12). Principal components were plotted for the rlog-transformed counts.

\section{Overrepresentation of GO terms in putative target genes}

The significantly differentially snatched genes (padj < 0.01) were investigated for overrepresentation of GO terms using R packages clusterProfiler (v 3.14.3,(Yu et al. 2012)) and GO.db (v2.9). Using the GO table as input a map including parent terms was build using buildGOmap. This gomap was used to build the required term2name table for the enricher and GSEA functions in clusterProfiler. Enricher was used to compare differentially snatched transcripts (padj < 0.01 and padj <0.05) against the universe of all snatched transcripts. In a second comparison, all snatched transcripts were compared against a universe of all GO annotated transcripts.

For GSEA all snatched genes were ranked using -log10(pvalue) * sign(log2FoldChange). GSEA was performed with fgsea with 1000 permutations and Benjamini-Hochberg multiple testing correction.

\section{KEGG pathway enrichment in snatched genes}

The snatched transcript IDs were converted to their RefSeq GeneID using the information contained in the genome annotation file. This list of genes was then used as input to the 
enrichKegg function with organism 'nta'. Capped host leader sequence transcript IDs were mapped to Arabidosis thaliana TAIR 10.1.

For the identified genes, the Ntab-TN90 protein sequence was mapped to the TAIR10.1 representative transcript amino acid sequence using blastp (-task blastp-fast -max-targetseqs 10 -outfmt '6 std qlen slen qcovs', ncbi-blast-2.5.0+, (Camacho et al. 2009). For each gene the best hit (lowest e-value) was selected where either \% identity and \% query coverage $>70 \%,>50 \%$ and $>80 \%$, or $>40 \%$ and $>90 \%$ respectively. The sequences with no hits or hits of insufficient quality were manually checked against A.thaliana and Solanacaea genes using NCBI blastp and gene IDs were mapped when query coverage of A.thaliana was similar to other Solanaceae coverage or other Solanaceae hits where annotated with an Arabidopsis gene ID.

For the pseudogenes, the RNA sequence was blasted using blastx against TAIR10.1 CDS sequences. IDs were mapped according to the same criteria as the genes plus those hits with an e-value of 0.0 .

All custom scripts are available on https://git.wur.nl/judith.risse/tswv

\section{Results}

\section{Identification of leader sequences through high throughput sequencing}

To characterize features of host cellular leader sequences at the 5' end of TSWV mRNAs, and collect possible clues that would hint to the cytoplasmic source from where these leader sequences were snatched, an HTS approach was used on non-viral leader sequences from $\mathrm{N}$ mRNAs. To be able, in addition, to compare a difference in the usage of host transcripts, plants were kept under standard conditions or subjected to heat in order to induce a (stronger) stress response. Plants were first infected with TSWV and at 7dpi, when the top leaves showed a systemic infection, one group was put at $37^{\circ} \mathrm{C}$ (heat treatment) while the other group was kept at standard conditions (M\&M; Fig. 5.1). After 3 hrs, systemically infected leaves were collected and used for RNA isolation (Fig. 5.1A), and subsequent 5 'race RT-PCR as shown (Fig. 5.1A) (Gu et al. 2015). N PCR fragments obtained, and containing heterogenous leader sequences, were purified and sent out for HTS analysis (Fig. 5.1B). From the sequencing data obtained, over 29,000 leader sequences were identified for each sample that mapped to a 5'UTR of $N$. tabacum genes (Table 5.1). 


\section{Chapter 5}

A

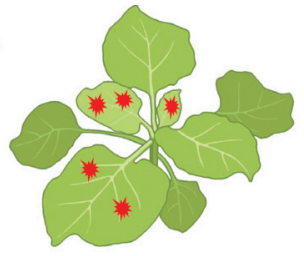

$23^{\circ} \mathrm{C}$

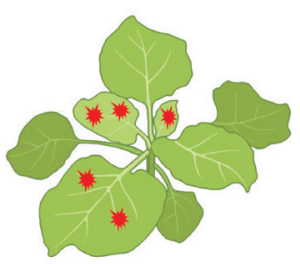

$37^{\circ} \mathrm{C}$

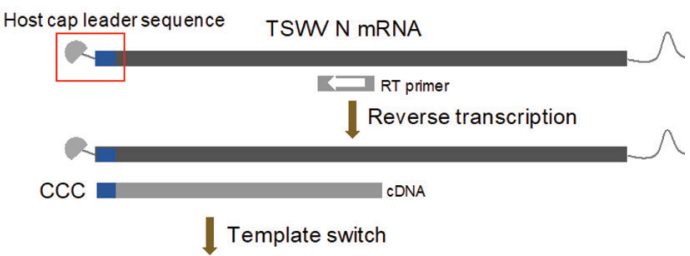

rGrGiG

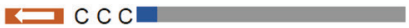

PCR

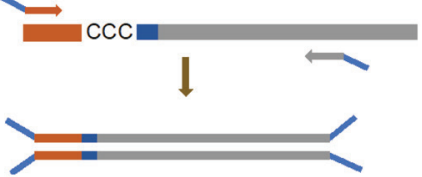

B

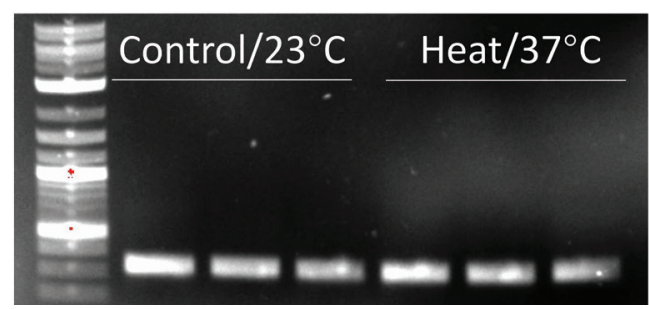

Figure 5.1 Workflow for the cloning and identification of host-derived 5' capped-RNA leader sequences of TSWV N mRNA. (A) Two groups of Nicotiana benthamiana plants were inoculated with TSWV and at 7 days post inoculation, one group was subjected to a heat treatment for 3 hours, after which systemically infected leaf samples were collected. Flow chart of 5'race RT-PCR after RNA isolation. (B)Agarose gel electrophoresis of the PCR amplified 5' end $\mathrm{N}$ gene fragment. First lane, DNA marker; Lane 2-4, replicate samples collected from leaves kept under normal condition; Lane 5-7, replicate samples collected from leaves subjected to a heat condition.

Table 5.1 Sequencing reads and mapping information.

\begin{tabular}{|c|c|c|c|c|c|c|}
\hline & $\begin{array}{l}\text { NG- } \\
27142 \_1-1 \\
\text { lib471243 }\end{array}$ & $\begin{array}{l}\text { NG- } \\
27142 \_2 \\
\text { lib471244 }\end{array}$ & $\begin{array}{l}\text { NG- } \\
27142 \_3- \\
\text { lib471245 }\end{array}$ & $\begin{array}{l}\text { NG- } \\
27142 \_4 \_- \\
\text {lib471246 }\end{array}$ & $\begin{array}{l}\text { NG- } \\
27142 \_5 \\
\text { lib471247 }\end{array}$ & $\begin{array}{l}\text { NG- } \\
27142 \_6 \\
\text { lib471248 }\end{array}$ \\
\hline treatment & control & control & control & heat & heat & heat \\
\hline read pairs & 139195 & 241440 & 175584 & 165576 & 133735 & 184051 \\
\hline trimmed & 97480 & 165537 & 117931 & 114347 & 89637 & 131597 \\
\hline merged \% & 60.3 & 60.2 & 59.2 & 61.3 & 62.2 & 60.7 \\
\hline aligned to TSWV & 96616 & 164228 & 117103 & 115326 & 88709 & 130336 \\
\hline putative CAP & 70535 & 124884 & 88411 & 75643 & 63563 & 95977 \\
\hline unique CAP & 32010 & 47618 & 38072 & 35922 & 30931 & 39418 \\
\hline mapped to 5' UTR & 64063 & 113670 & 80508 & 67523 & 57109 & 86861 \\
\hline mapped in same strand & 30840 & 58027 & 38121 & 29839 & 26789 & 42737 \\
\hline
\end{tabular}




\section{Characterization of leader sequences}

To investigate the presence of sequence motifs and features within the host-derived leader sequences, first the size distribution of these sequences was analysed. When a 2 nt overlap with the viral template sequence was included, the sequences ranged in size between 12-27 nt, with the highest abundancy of leaders ranging between 16-20nt (Fig. 5.2A). Most of the leader sequences end with AGA or GAG (Fig. 5.2B). To investigate if TSWV prefers certain donor RNA sequences for cap-snatching, leader sequences were analysed for conserved motif conservativity and the snatched chance of highly enriched motifs. In Fig. 5.2C, sequences (motifs) that appeared in highest amounts in the population of host leaders are listed. However, analysis of these motifs, like in the case of snRNA-derived leader sequences in Influenza viral mRNAs, did not point to a certain class of cap-donors, or foci from where TSWV preferentially used capped leader sequences.

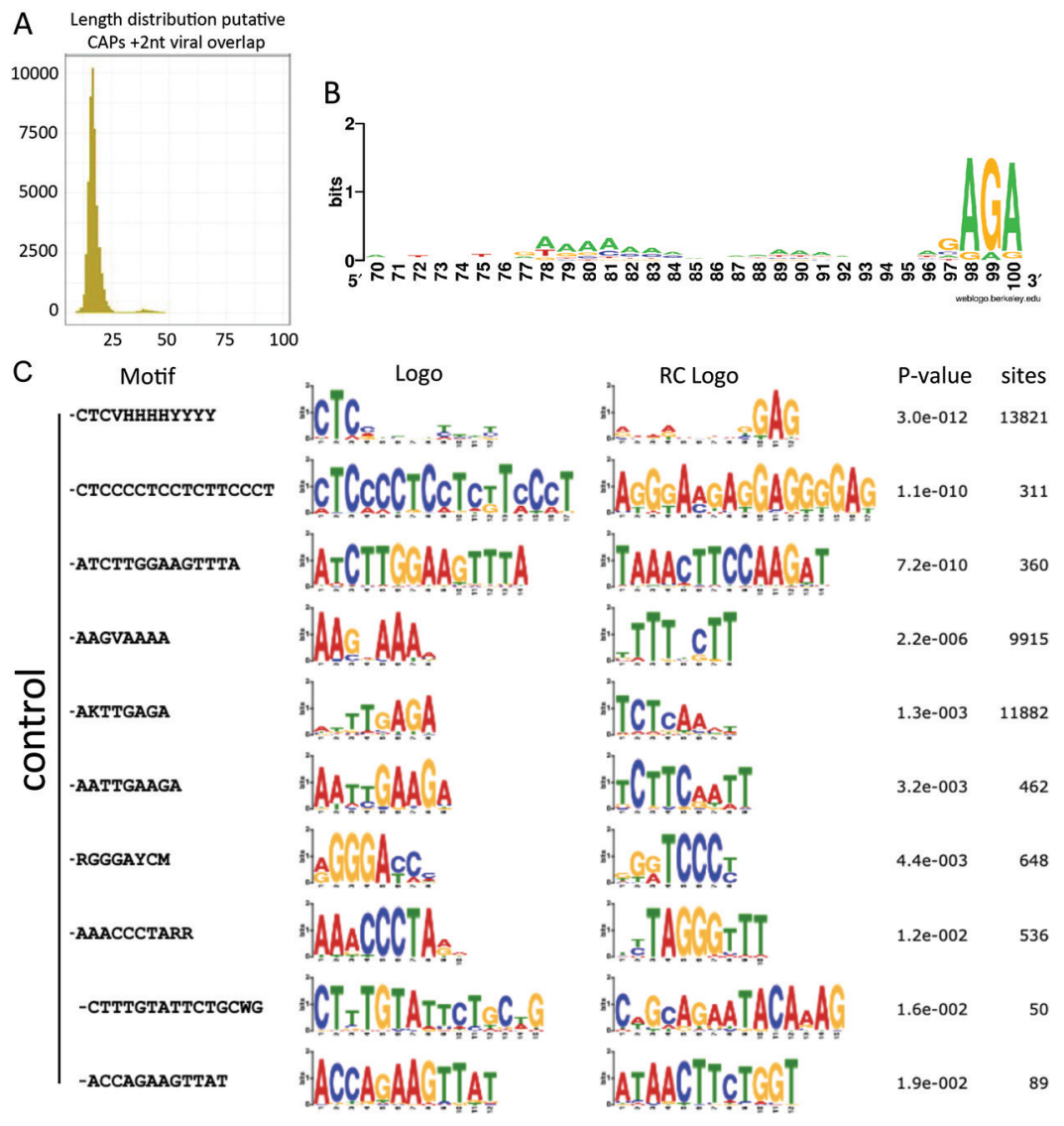



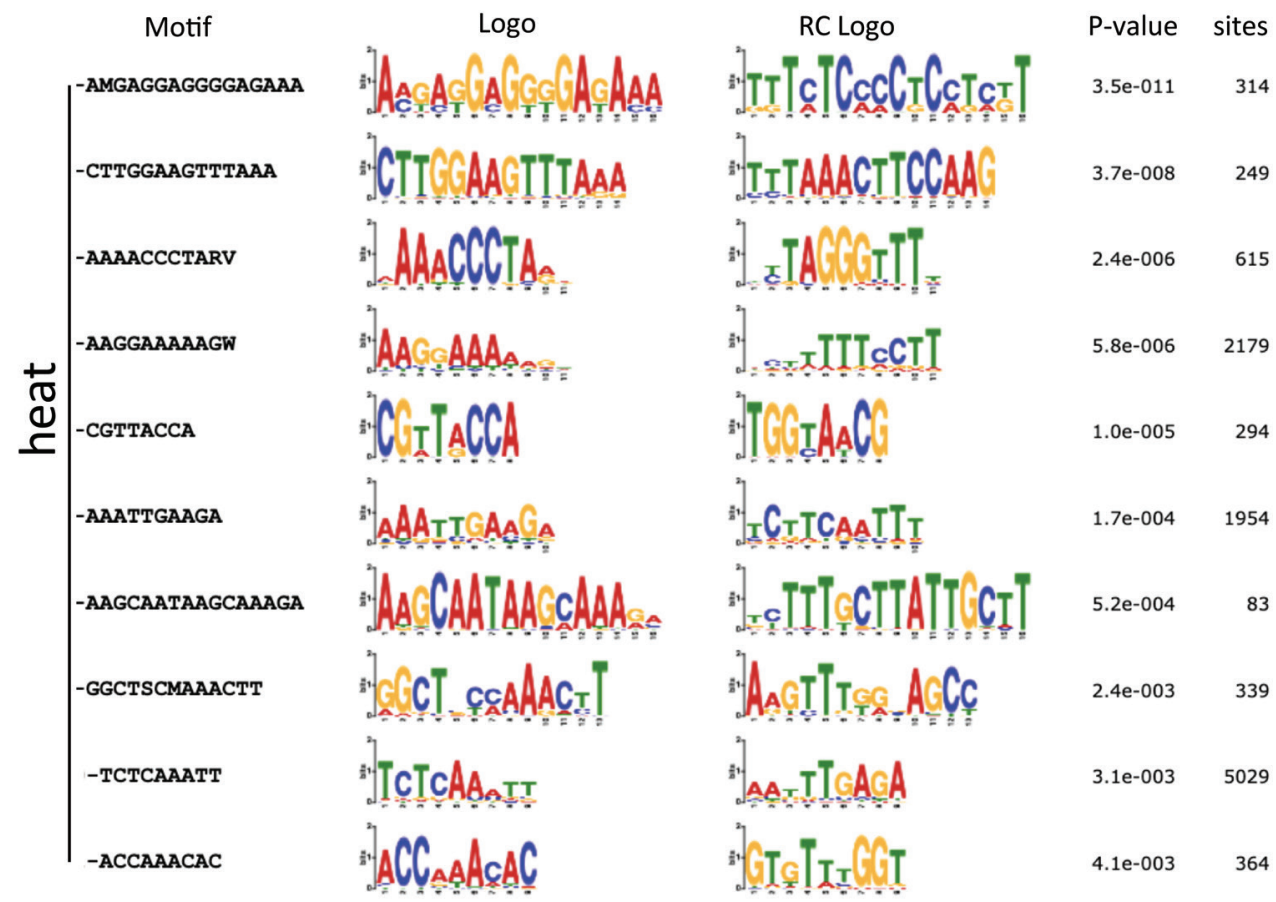

Figure 5.2 Sequence motifs and features of capped leader sequences. (A) Size distribution analysis of capped leader sequences from TSWV N mRNAs. (B) Nucleotide sequence analysis at the junction of non-viral and viral sequence (reflecting the cleavage site in host cellular mRNA leaders). (C) Motif analysis of capped leader sequences for different group of samples. Each logo consists of stacks of symbols, one stack for each position ( $X$ axis) in the sequence. The overall height ( $Y$ axis) of the stack indicates the sequence conservation at that position, while the height of symbols within the stack indicates the relative frequency of each amino or nucleic acid at that position.

\section{Identification of DEGs that are being used during cap-snatching at normal and heat stress conditions}

Having analysed and characterized features from the snatched leader sequences, next an attempt was made to identify the corresponding genes from which the leaders were snatched. To see if there are differences in the use of different transcripts, the frequency of donor transcript usage in normal and heat-treated samples was analysed. Prior to this, a principle component analysis was performed to analyse the correlation between the six samples. The first principle component explains $58 \%$ of the variance between the samples and clearly separates the two groups. The results represented a clearly different distribution of transcripts at normal condition and heat stress condition (Fig. 5.3A). Hierarchical clustering analysis also showed the formation of two distinct groups, 
A

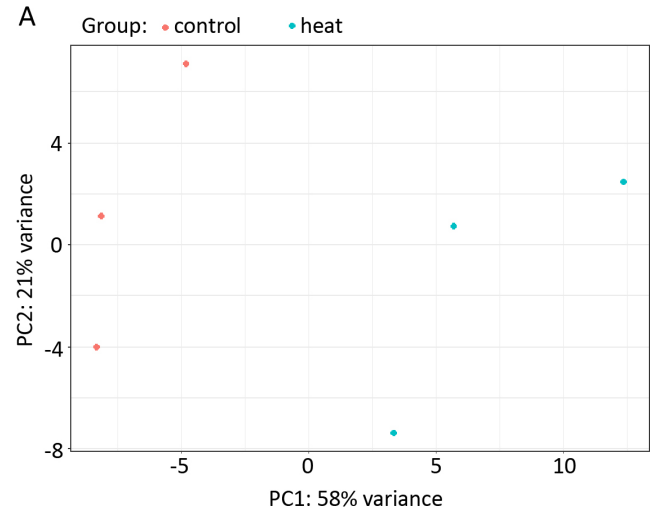

B

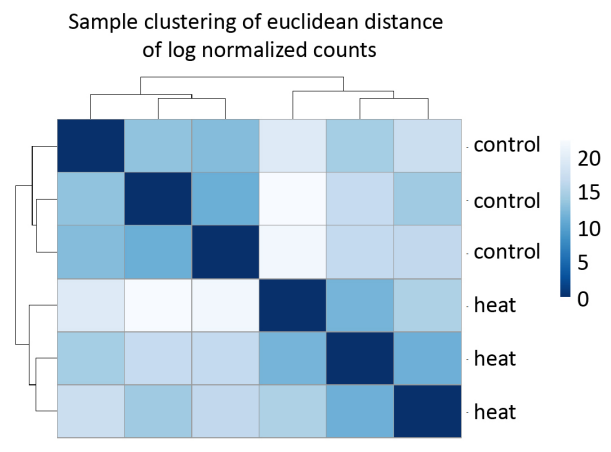

C

C Differentially snatched capped leader host genes with $\mathrm{p}$ adj $<0.01$

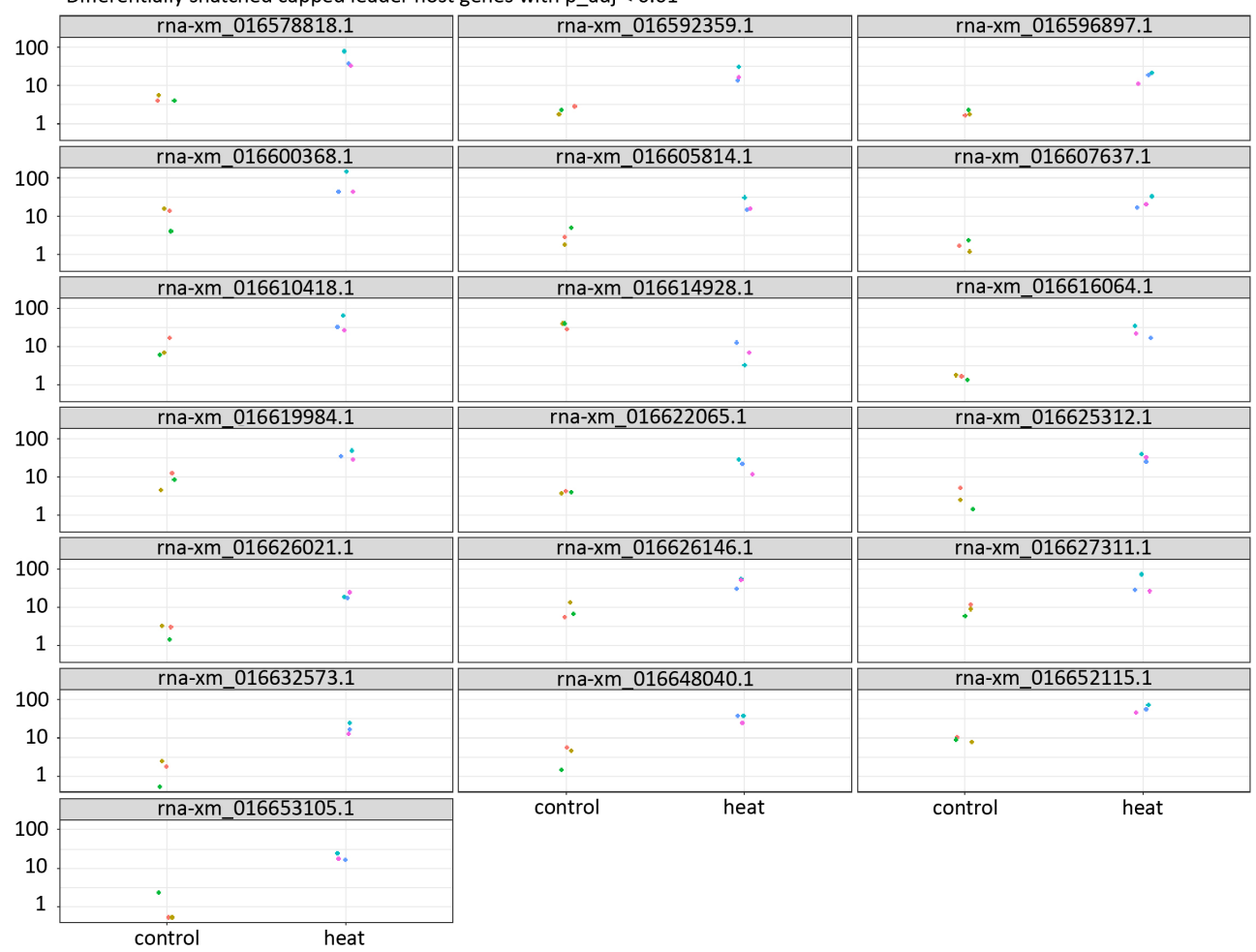

Figure 5.3 Global Evaluation of the cap-seq samples. (A) PCA analysis of samples from the two different treatments. (B) Hierarchical clustering of the six samples and showing their biological variation. (C) Total overview of the 19 differentially snatched genes, at normal and heat stress conditions.

representing the samples collected at normal condition clustering in one group versus the other group containing the samples that were subjected to a heat treatment (Fig. 5.3B). 


\section{Chapter 5}

Having verified and confirmed the homogeneity/quality of the triplicate samples, next, all host genes identified, from where the snatched leader sequences were collected, were analysed on their expression profile. Transcripts from 18 of those genes were differentially used between the two conditions (FDR adjusted p-value < 0.01, Fig. 5.3C). Most of them were more used at heat stress condition while only one of them was less used.

\section{An overview of identified genes encoding cap donor transcripts by GO enrichment analysis and KEGG pathway analysis}

In a next step, the identified host gene transcripts and their source genes were analysed. Of all these, 348 transcripts (from 330 different genes) were identified with 10 or more reads. A GO enrichment of these genes compared to all other genes with $\mathrm{GO}$ annotation revealed that 28 terms were significantly overrepresented (Fig. 5.4A) ( $p<0.01$ ). The top 10 enriched GO biological processes were photosynthesis, generation of precursor metabolites and energy, photosynthesis light reaction, photosynthesis light harvesting, thylakoid, photosystem, photosynthetic membrane, thylakoid part, photosystem II and cellular carbohydrate biosynthesis (Fig. 5.4A).

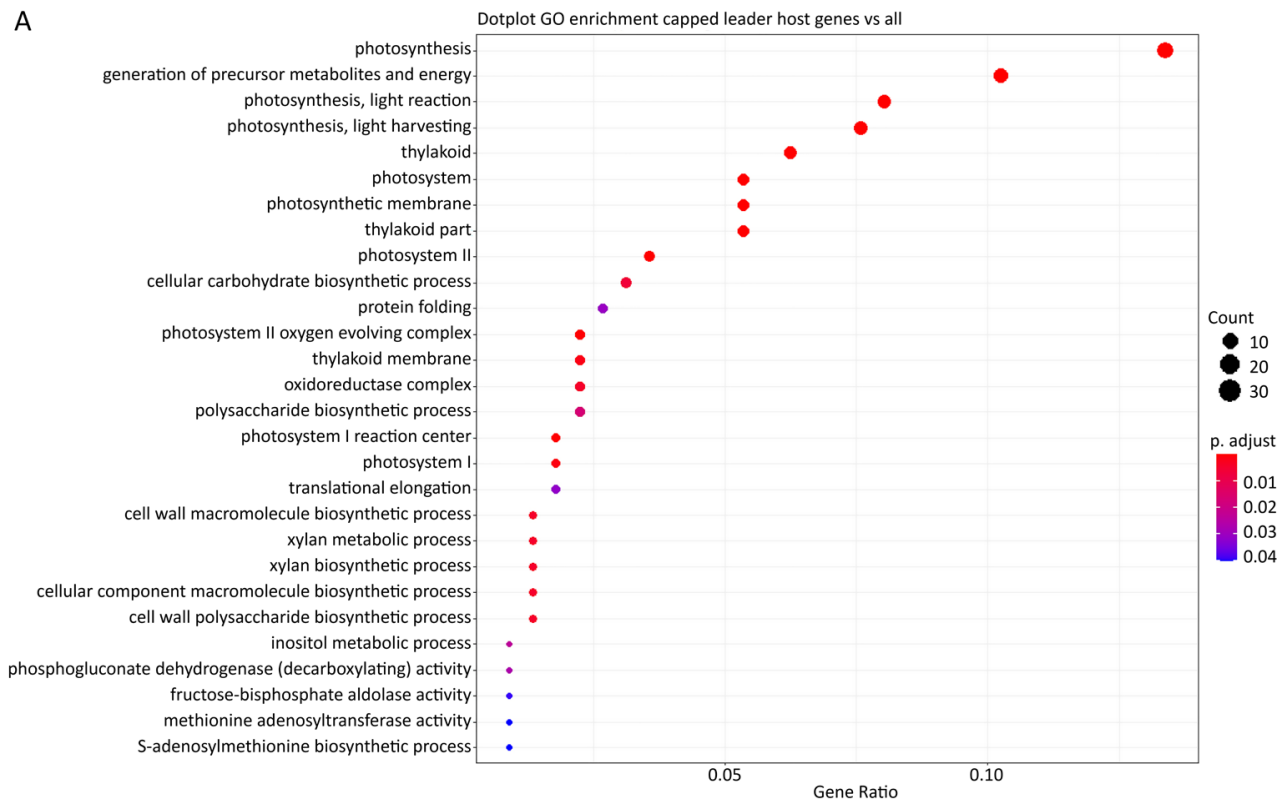

Figure 5.4 GO Enrichment and KEGG pathway analysis of cap donor genes. (A) Overview on significantly enriched pathways by $\mathrm{GO}$ enrichment analysis. 
B Overrepresented GO terms in capped leader host genes (padj $<0.01$

protein folding

oxidoreductase complex

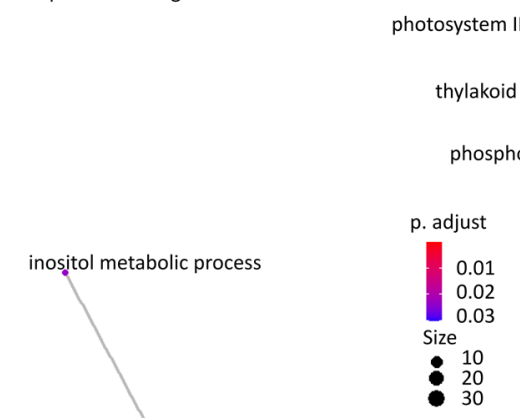

cellular carbohydrate biosynthetic process

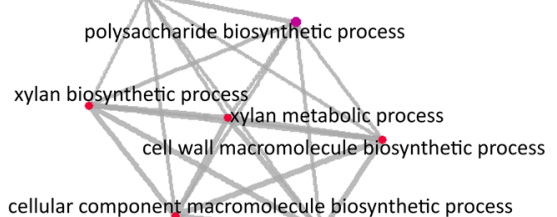

xygen evolving complex

photosystem I reaction center

$1 \mathrm{x} x$

photosynthetic membrane

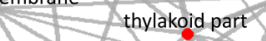

gluconate dehydrogenase (decarboxylating) activity

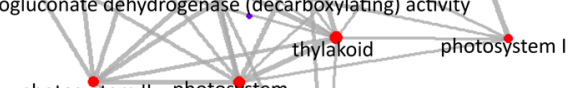

photosystem II photosystem
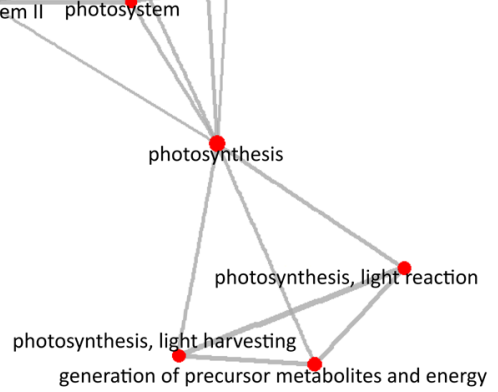

cellular component macromolecule biosynthetic process

cell wall polysaccharide biosynthetic process

C Overrepresented KEGG pathways in capped leader host genes
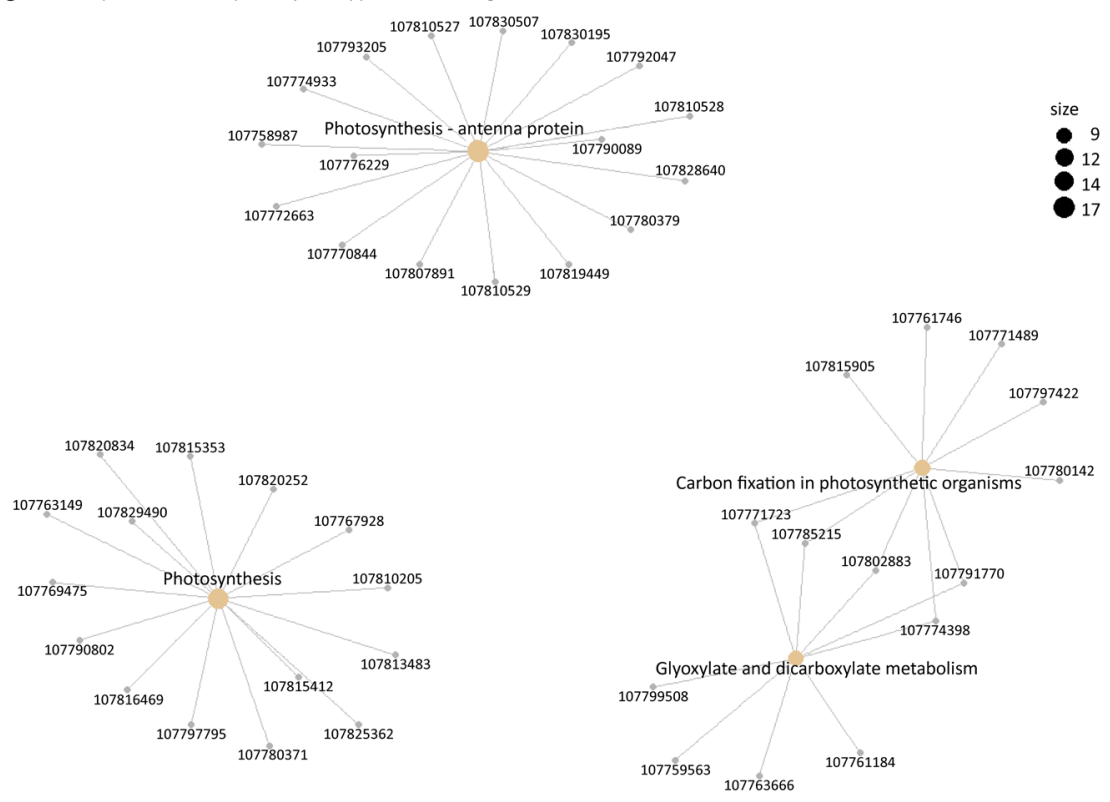

Figure 5.4 GO Enrichment and KEGG pathway analysis of cap donor genes. (B) Schematical diagrams of networks from significantly enriched pathways. (C) Significantly enriched pathways by KEGG pathway analysis. 
After this, the significantly represented pathways were classified into networks. In total, this yielded 3 connected networks (Fig. 5.4B). These networks covered fourteen photosynthesis pathways in one network, eight cellular biosynthetic pathways into a second connected network and a third one in protein folding. When a KEGG pathway enrichment analysis was performed on the identified genes from the cap donor transcripts, four pathway terms with the greatest enrichment were identified, namely "photosynthesis-antenna proteins", "photosynthesis", "glyoxylate dicarboxylate metabolism" and "carbon fixation in photosynthetic organisms" (Fig. 5.4C).

\section{Both functional and non-functional transcripts are used for cap-snatching}

Based on the HTS results and the gene transcripts identified as cap donor, it was not possible to determine whether transcripts containing a PTC were being used. Transcripts containing a PTC are thought to enter the NMD pathway and are assumed to traffic to P bodies for further degradation. In order to analyse a potential preference of TSWV for the usage of functional transcripts or non-functional (PTC containing) transcripts during cap-snatching, a cap donor competition experiment was performed. To this end, a nonsense (ns)GFP construct containing a PTC right downstream of the start codon was generated (Kim and Maquat 2019)(Fig. 5.5A). An identical, but functional GFP construct (lacking the PTC) was used as a control. To be able to distinguish between leaders snatched from the functional GFP or nsGFP transcripts, a marker nucleotide was introduced at nt position 12 (Fig. 5.5A). To test the relative usage of both functional and non-functional transcripts, GFP and nsGFP constructs were infiltrated to TSWV infected plants in a 1:1 ratio (Fig. 5.5A). Leaf samples were collected and total RNA purified to be used for a $5^{\prime}$ race PCR to clone capped leader sequences from TSWV N mRNA (Fig. 5.5A and 5A). PCR fragments obtained, and of expected size, were purified and ligated into pGEM-T easy vector for sequencing. From the 55 positive clones collected, 35 contained the leader sequence from GFP and 20 from nsGFP, as identified by the marker nucleotide (Fig. 5.5B). The expression of both transcripts was verified by qRTPCR, and revealed that the level of GFP transcripts was about two times higher than that of nsGFP (Fig. 5.5C). Despite these differences, the results altogether indicate that both functional GFP and non-functional GFP transcripts are used during TSWV cap-snatching, and there seems to be no strong preference for either transcript, when somewhat corrected for their expression levels. 

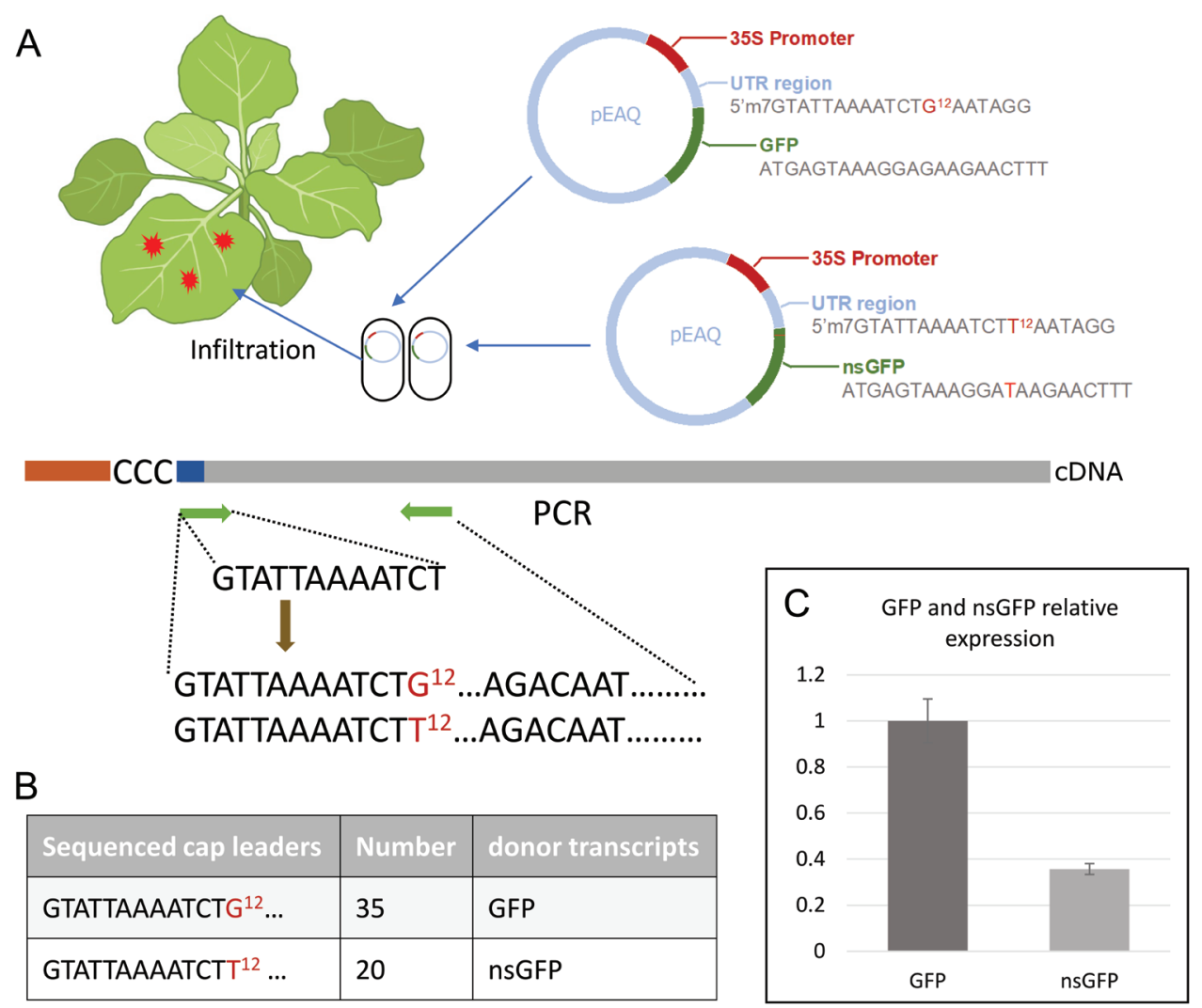

Figure 5.5 Cap donor competition assays of functional GFP and non-functional GFP. (A) Schematical diagram of GFP and nsGFP constructs, agrobacterium infiltration on the TSWV inoculated leaf at 5dpi TSWV cleavage strategy for GFP/nsGFP transcripts and cloning strategy of PCR fragment with GFP/nsGFP capped leader sequence. (B) Number calculation of capped leader sequences from GFP/nsGFP (C) qRT-PCR on the expression of GFP/nsGFP.

\section{The usage of GFP and long GFP transcripts is altered by heat stress}

As known from previous studies, plants subjected to heat stress undergo transcriptional reprogramming and stress granule (SG) formation (Guo et al. 2016; Hamada et al. 2018). This also leads to functional transcripts becoming stalled in translation and stored at SGs (Khong et al. 2017). Several studies, though, suggest that this especially applies to long transcripts. Results from the HTS analysis did reveal a difference in the usage of certain transcripts at normal and heat conditions, and could imply a relocalization of those transcripts (Fig. 5.3). However, and alternatively, it could also be caused by heat induced gene expression changes. To further investigate if short-term heat stress and related SG formation affects 


\section{Chapter 5}

transcript usage during TSWV cap-snatching, TSWV-infected plants were co-infiltrated with two constructs encoding a normally sized functional GFP (0.7 kb) or and long (L)GFP $(6.9 \mathrm{~kb})$ transcript to test their usage as cap donor in a pairwise (1:1) competition. After coinfiltration of both constructs, plants were placed at normal $\left(23^{\circ} \mathrm{C}\right)$ and heat stress $\left(37^{\circ} \mathrm{C}\right)$ conditions, respectively (Fig. 5.6A). According to other studies, SG enriched transcripts are relatively long with an average size of $7.1 \mathrm{~kb}$ while short, translatable transcripts do not accumulate in SG (Khong et al. 2017). Based on this, the long GFP transcript was expected to accumulate at SG under heat stress conditions. If TSWV would indeed use cap donors from SG, an increased usage of the leader sequence from the LGFP transcripts was expected in the samples subjected to heat stress. When viral $\mathrm{N}$ gene transcripts were amplified by the 5 'race RT-PCR, and the obtained fragments cloned, the usage of GFP and LGFP as cap donors was determined by the presence of the marker nucleotide introduced in the leader sequence at nucleotide position 12 . From the 35 clones obtained from plants kept at $23^{\circ} \mathrm{C}$, 26 leader sequences were from the GFP transcript and 9 were from the LGFP transcript. However, from the 30 clones obtained from the samples subjected to a heat stress, 16 contained a leader sequence originating from GFP while 14 were from LGFP, suggesting an increased usage of the LGFP cap donor under heat stress conditions (Fig. 5.6B).

A

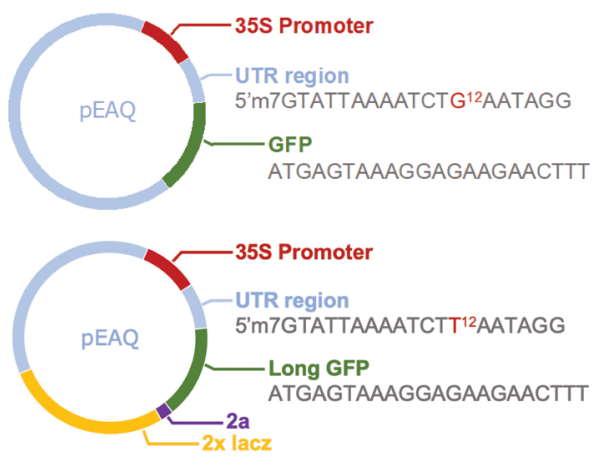

B

\begin{tabular}{|l|l|l|}
\hline Sequenced cap leaders & Number & donor transcripts \\
\hline GTATTAAAATCTG $^{12} \ldots$ & 26 & GFP \\
\hline GTATTAAAATCTT $^{12} \ldots$ & 9 & LGFP \\
\hline GTATTAAAATCTG $^{12} \ldots$ & 16 & GFP \\
\hline GTATTAAAATCTT $^{12} \ldots$ & 14 & LGFP \\
\hline
\end{tabular}

Figure 5.6 Cap donor competition assays of functional GFP and long GFP. (A) Schematical diagram of GFP and nsGFP constructs. (B) Number calculation of capped leader sequences from GFP/L-GFP at different conditions (C) qRT-PCR on the expression of GFP/L-GFP at different conditions. 
To verify the expression level of both transcripts at different conditions, a qRT-PCR was performed, which showed that at normal $\left(23^{\circ} \mathrm{C}\right)$ conditions the expression level of GFP was much higher than from LGFP (Fig. 5.6C). Heat stress significantly reduced the GFP expression level while hardly affecting LGFP expression. At $23^{\circ} \mathrm{C}$, the GFP expression level was 8.9fold higher than that of LGFP, while under heat stress it was only 4-fold higher (Fig. 5.6C). Altogether, these results indicated that both GFP and LGFP transcripts were used during TSWV cap-snatching and heat stress (inducing SG formation and storing long transcripts stalled in translation) seems to affect the usage of different transcripts.

\section{Discussion}

So far, the source of cap donors for the cytoplasmic-replicating NSVs remains to be elucidated. Previous studies have indicated that $\mathrm{P}$ bodies may provide in (degradable) capped RNAs for cap-snatching(Hopkins et al. 2013; Mir et al. 2008b). However, results from studies described in this thesis (Chapter 4 ), also pointed to the possible role of stress granules (SGs) and nucleopore mRNA export in providing capped RNAs. In this study, HTS was used to sequence a large amounts of host-derived cellular mRNA leader sequences at the $5^{\prime}$ end of TSWV N mRNAs to analyse for certain motifs and sequence features, as well as to identify the corresponding host donor genes. In addition, cap donor competition assays were performed to verify the usage of transcripts destined for normal translation (GFP), to $P$ bodies (nsGFP) mediated by the nonsense mediated degradation (NMD) pathway of PTC transcripts, or to SG (LGFP) upon heat stress induction. After bioinformatical analysis of the HTS data 330 genes were identified whose transcripts were used as cap donor by TSWV, and they were highly enriched in genes from the photosynthesis pathways. Heat treatment/stress induction clearly influenced, and changed the usage of host transcripts. Analysis of all snatched leader sequences did not reveal specific motifs or features that hinted towards the origin of a specific cap donor source. Further studies using cap donor pair wise competition assays on the other hand, showed that TSWV used transcripts of functional GFP, GFP with a PTC as cap donors, although to a different extent. These results all together indicate that TSWV snatches capped leader sequences from a variety of transcripts, and from different sources.

Earlier studies on snatched leader sequences by Influenza virus revealed motifs that pointed to the snatching of capped RNA leaders from snRNAs, pointing to the usage of cap donors from Uridine-rich small nuclear ribonucleoproteins (U snRNPs) that are involved in key steps 
of pre-mRNA processing (Refs). For leaders from TSWV N transcripts no specific motifs or features were found. Although these could have pointed to a source, alternatively motifs could also point to host proteins that bind to these motifs and could as a scaffold for docking of a TSWV transcriptase complex. On this point certain classes of mRNA are known to contain motifs in their 5'UTR to bind heterogeneous nuclear ribonucleoproteins (Chang et al. 2017; Gui et al. 2010; Lee et al. 2010; Li et al. 2018a; Ng et al. 2005; Shi et al. 2003; Wang et al. 2014).

The findings that TSWV likely snatches leader sequences from a variety of transcripts, and from different sources seem to contrast those reported for the hantavirus SNV in an earlier study by Mir et al. (2008), where a colocalization of SNV N protein solely with P bodies was observed, and the virus appeared to show preference for PTC transcripts during transcription. The reason for a sole colocalization to $\mathrm{P}$ bodies is strange, seeing that in studies as described in this thesis (Chapter 4) the SNV N protein, expressed from the same construct, ended up colocalizing with SG as well. Furthermore, in contrast to the pair wise in vivo cap donor competition assays as performed here, using either GFP and nsGFP or GFP and LGFP under both normal and heat stress (SG induction) conditions, the studies by Mir et al (2008) only tested a GFP-PTC or functional GFP transcripts separately and not in a pair wise competition. In addition, in cells depleted from PB, SNV transcription-replication levels were not affected, nor the use of GFP-PTC transcripts, which led the authors to propose that SNV likely could target GFP-PTC transcripts earlier, before they reached PB. The observations in our study support this idea, as transcripts targeting PB and SG granules, but also normal GFP transcripts (engaged in translation) are still being used as cap donors under normal and heat stress conditions, although some slight differences are observed. For these reasons, it slowly becomes appealing that these viruses likely use cap donors upstream, e.g. at the Nuclear pore complex (NPC), as postulated in Chapter 4, and supported by silencing of elements related to the NPC and nuclear mRNA export pathway. Collecting capped leaders from there would then likely occur randomly and leaders found at the 5' end of viral mRNAs genuinely reflect those from the pool of mRNAs that pass the NPC into the cytosol from where many engage either in (polysomal) translation, or move onwards to PB or SG, depending on ((a) biotic) conditions.

Support for this idea also comes from the HTS analyses of snatched leader sequences for viral mRNA transcription, and the cellular mRNA origin of these (this chapter), reflecting the pool of cellular transcripts observed during transcriptome analysis on TSWV infected plants 
(Chapter 3). The HTS approach as applied in this chapter allowed the identification of 29,000 capped leader sequences of which most agreed to the requirements as earlier reported for leaders used during TSWV cap snatching (Duijsings et al. 1999, 2001). This support the HTS approach as being a powerful tool for massive capped RNA leader identification, to analyse for the presence of motifs or sequence features within highly enriched capped leader sequences found in viral mRNAs. The presence of those features will help in the identification of binding host proteins that could assist in cap-snatching, e.g. in the recognition/binding of capped RNA leader sequences from host cellular mRNAs. While most reads conform to the expected pattern of polyG_host-leader-sequence_TSWV (polyG, referring to the template switch primer used for the amplification of leader sequences), and are mapping to 5'UTR sequences of $N$. tabacum, only about $50 \%$ of those mapped in the same polarity as the transcript, while the others mapped to the reverse sequence of the UTR were removed from further analysis. This is mostly due to the shortness of the leader sequences increasing the chance of random alignments. However, it also means that those sequences should map to the genome upstream of a gene outside the currently annotated or inferred 5'UTR regions. Another issue that complicates the mapping is that a cross-species mapping has been performed, i.e. N. benthamiana host leader sequences were mapped onto N. tabacum, enforced by the poor annotation state of $N$. benthamiana. A number of host capped leader sequences might thus not correctly map when the two species differ in certain regions.

The identification of hundreds of donor genes revealed that TSWV uses many different cellular gene transcripts for cap-snatching. GO enrichment analysis and KEGG pathway analysis showed many of those genes are involved in the photosynthesis pathway. This echoes with our previous RNA-seq data from TSWV infected Arabidopsis where a major down-regulation was observed of genes related to the photosynthesis pathway (Chapter 3 ), suggesting that their transcriptional repression may likely have resulted from cap snatching. Many plant viruses cause chlorosis and/or yellowing symptoms on host plants via modulation of the photosynthetic pathway. Changes include inhibition of photosystem efficiency, changes in chloroplast structure, fluctuation in chlorophyll fluorescence, imbalanced accumulation of photo assimilates, transcriptional repression of photosynthetic genes, interference at protein binding with chloroplast factors, and so on, altogether contributing to a chlorosis phenotype (Zhao et al. 2016). Transcriptional repression of photosynthesis related genes has also been observed for Cucumber mosaic virus (CMV), Plum pox potyvirus (PPV), Tomato ringspot nepovirus (ToRSV), and Prunus necrotic ringspot ilarvirus (PNRSV) (Dardick 2007; Mochizuki et al. 2014). Due to these viruses differing in their genome structure, their replication and 
transcription strategies, the causes of such down-regulation might be different. For some viruses, though, the photosynthesis pathway is essential for viral uncoating and replication (Ahlquist et al. 2003; Dreher 2004; Torres et al. 2006; Xiang et al. 2006). Whether, in the latter cases, the interplay of viral uncoating and the photosynthesis pathway is related to the development of disease symptoms was not investigated nor discussed.

Heat is a type of (abiotic) stress for plants of which a consistent treatment up to a certain threshold induces transcriptional reprogramming and stress granule formation (Guo et al. 2016; Hamada et al. 2018). Previous experiments have shown stress granule formation under heat treatment and during which the N protein of cytoplasmic-replicating NSVs also colocalizes to these condensates (Chapter 4), providing support for a possible role of SGs as source of capped leaders for cap-snatching. In this study, the usage of long GFP transcripts, relative to normal GFP transcripts, increases upon heat stress induction, a condition that is known to specifically stall long transcripts in translation at SG. This result indicates that transcripts temporarily concentrated in SG can be snatched by cytoplasmic-replicating NSVs' during transcription initiation. Moreover, these transcripts are more efficiently used at stress condition, and maybe also more stabilized due to the condensation and leading to a longer turnover time. During the HTS analysis of differentially used transcripts, also 17 transcripts were identified that were more efficiently used after heat stress induction compared to the normal condition, while only 1 was less used. This also indicates that heat stress does change the usage for certain transcripts. Although no certain pathways were identified for those host gene transcripts used as cap donor, their increased usage after heat induction likely relates to a change in their spatial distribution/availability in granules, probably due to storage in SGs. Conversely, and complementing the studies on the LGFP transcript usage after heat stress induction, it provides support for SGs presenting a possible source of cap donors for the cytoplasmic replicating NSVs. Of course, one cannot exclude that heat stress increases the expression of some proteins e.g. heat shock related proteins, which are increased by heat stress, to response to the protein misfolding, translocation, degradation (Guo et al. 2016; Schöffl et al. 1998). There could also be an increased usage in heat related transcripts.

Despite the usefulness of the HTS approach, it was not possible to distinguish which of the transcripts used were functional or not, which would have supported studies to indicate the role of PB and SG in cap snatching. Still, and altogether, the findings indicate that TSWV may use a large number of various transcripts (functional, non-functional, long), directly 
accessed from the nucleocytoplasmic export pathway at NPC, irrespective of their final cytoplasmic destination. Downstream (PB/SG) RNA granules may still be used, but might act as a supplemental source for cap-snatching, as supported by their redundancy during silencing of some RNA granule components.

\section{Acknowledgements}

We would like to thank Prof. dr. Monique van Oers (Wageningen University) for critically reading the manuscript prior to submission. 

Chapter 6

\section{General discussion}

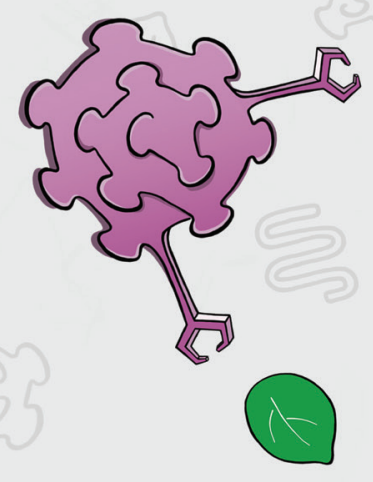

Min Xu
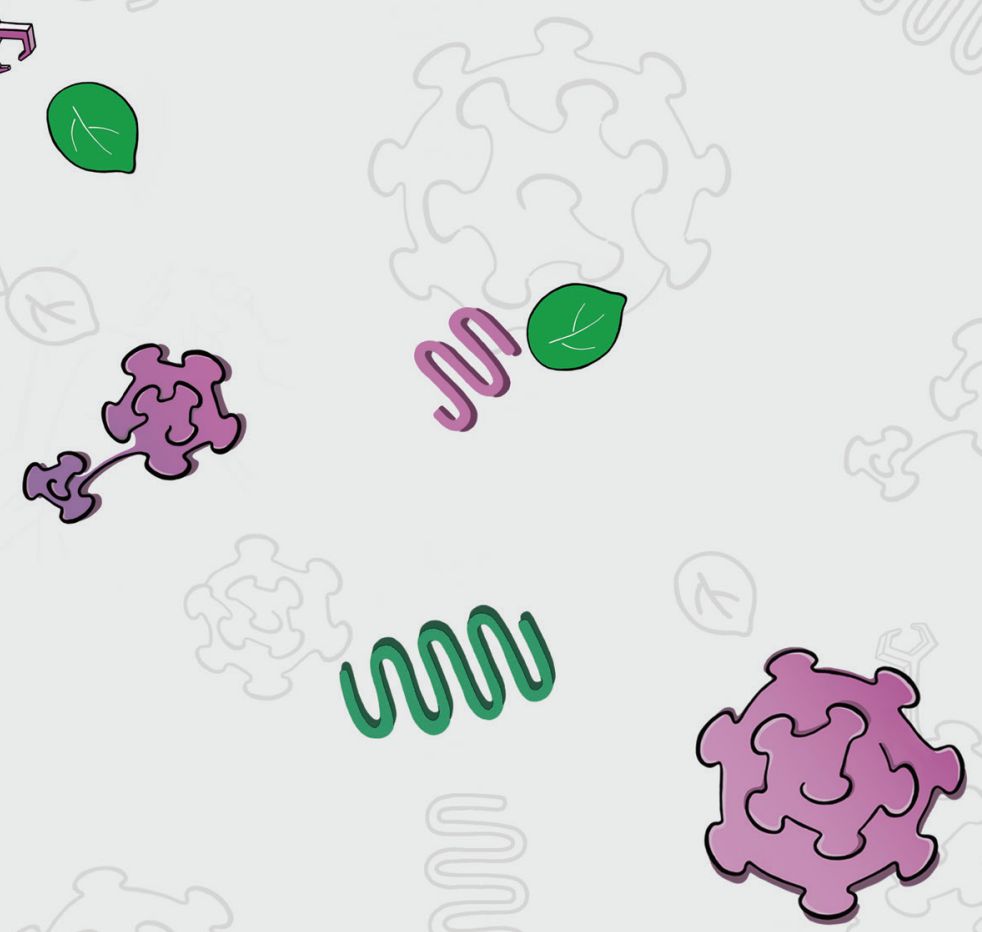
Cap-snatching is a common and highly conserved mechanism used by most segmented, negative stranded RNA viruses (NSVs) to initiate viral genome transcription. Since the discovery of cap-snatching in the early 70's of the past century, knowledge of this mechanism has grown impressively. Many features of cap-snatching have been identified for both the nuclear-replicating influenza viruses and cytoplasmic-replicating bunyaviruses. Although they differ in their site of replication, so far, most features unravelled are generic and similar for both groups of viruses. The mechanism and the role of the viral polymerase in capsnatching has been more extensively studied with Influenza viruses and these studies led, amongst others, to the identification of RNA polymerase II transcription sites as the nuclear source from where Influenza takes capped-RNA leader sequences to initiate viral genome transcription. In contrast, and the enigma from where the study described in this thesis embarked, it is not known from which cytoplasmic sources of mRNA (granules/pools//foci) the cytoplasmic replicating bunyaviruses snatch capped RNA leader sequences, nor is the exact role of the viral RNA dependent RNA polymerase (RdRp) and N proteins in this process understood.

To this end, and as described in the previous chapters, various molecular, biochemical, bioinformatical and cell biological experiments have been performed. The results from this thesis research show that the $N$ proteins from several distinct bunyaviruses associate to $P$ bodies (PBs) and stress granules (SGs) and indicates that these viruses might use capped RNA leader sequences from both cytoplasmic condensations. Even the cytoplasmic site of the Nuclear Pore Complex (NPC), that receives a constant supply of mRNA from the nuclear efflux, turned out to present a potential source for cap donors. Silencing of elements from these condensations/foci, affected levels of viral replication, thereby providing support for their potential candidacy as source of capped RNA. Some of the observations were made, and strengthened, by the analysis that included plant- and animal infecting bunyaviruses. High Throughput Sequencing (HTS) of host cap leader sequences collected from normal, and (heat-) stress conditioned infected plants, revealed changes in the dynamics and differential usage of host gene transcripts for cap-snatching, and further strengthened an interplay with other pools beyond $\mathrm{P}$ bodies, e.g. with stress granules.

Although the studies described in this thesis have not fully solved the enigma on the cytoplasmic source(s) (granules/foci/pools) of mRNAs from where the cytoplasmic replicating bunyaviruses snatch capped-RNA leaders, the findings described in this thesis will be helpful to design follow up experiments to answer the remaining questions. E.g. 
what makes NPC an interesting candidate as source for capped RNA-leaders? What are the mechanisms by which the (cytoplasmic) mRNAs are recognized by all these viruses? How would these viruses preferentially hijack mRNAs with premature stop codon (PTC), as reported in a few studies, or are these observations to be explained in another way? Have we been blinded by the visibility and constant presence of $\mathrm{P}$ bodies, and thereby ignoring the possibility of other more transient, less visible pools/sites of cytoplasmic mRNAs for cap-snatching? In the following sections a model is presented to discuss the findings of this thesis research in a cell biological context and some perspectives is given on several of the aforementioned unsolved and challenging questions.

\section{All the way, and down the road of RNA destiny: Possible cytoplasmic sources of host cellular mRNA for bunyavirus cap-snatching}

Previous data have pointed to $\mathrm{P}$ bodies as the cytoplasmic pools for capped RNA in hantavirus cap-snatching (Cheng and Mir 2012; Mir et al. 2008b; Mir et al. 2010), and a few follow up studies further supported this idea (Hopkins et al. 2013; Ma et al. 2019). Data presented in this thesis indicate that these viruses likely are not limited to P bodies, and SG but also NPCS could present potential sources (Chapter 4). In vivo cap donor competition assays during TSWV transcription, using functional GFP transcripts and GFP-PTC transcripts (destined for RNA degradation), revealed that also functional GFP transcripts are very well-used during viral transcription (Chapter 5). These findings indicate that the virus has the possibility to snatch capped-RNA leaders from transcripts engaged in (cytoplasmic/polysomal) translation, and that these do not seem to be outcompeted by transcripts entering the nonsense-mediated decay (NMD) pathway, and traffic to PBs (Chapter 4). Earlier studies on Sin nombre virus (SNV) cap snatching also indicated that this virus may already collect capped RNA leader sequences from PTC transcripts before they reach PBs (Cheng and Mir 2012). These results altogether indicate that the virus already (and maybe also likely) has the ability to snatch capped leader sequences from mRNAs upstream on the RNA-destiny road that leads to $P$ bodies/stress granules.

In Figure 6.1 the cytoplasmic trafficking route of (m)RNA, starting at the NPC, is depicted, to overview possible sources of capped-RNA that can be used for bunyavirus cap-snatching. In general, two major classes of capped RNAs can be distinguished in this pathway. The first one is the major class of host cellular mRNAs produced in the nucleus and exported to the cytoplasm for further translation and use in cellular processes. The second class presents 


\section{Chapter 6}

capped, small nuclear RNAs (snRNAs) which function in mRNA processing. SnRNPs assemble and mature cytoplasmically into $U$ bodies, after which they return to the nucleus for functionality (Liu and Gall 2007). During their maturation (and storage), U bodies associate with $P$ bodies. When bunyaviruses would use any cytoplasmic source of capped RNA, one would also expect to see a co-localization of $\mathrm{N}$ with $\mathrm{U}$ bodies. Not entirely surprising, localization studies with Schmallenberg virus (SBV) N protein also revealed a co-localization with cytoplasmic $U$ bodies, although the amounts were relatively minor. Most striking and interesting though, were the observations on the perinuclear localization of the $\mathrm{N}$ protein to NPC, and the observed "detrimental" effects on Tomato spotted wilt virus (TSWV) replication after silencing RanGAP, an important factor in nuclear mRNA export. The observations in this thesis altogether point to the possibility that bunyaviruses may access cap donor RNAs at NPCs (Fig. 6.1, point 1), at pre-translational and translational stages (Fig.6.1, point 2), and at RNA granules at the end of the road of RNA destiny (Fig.6.1, point 3). There is no reason to argue why this would not be possible, although it is tempting to speculate on a highly preferred source. Whether this is presented by PBs/SGs, or is to be found at NPCs, the start of the cytoplasmic trafficking road, needs further investigation.

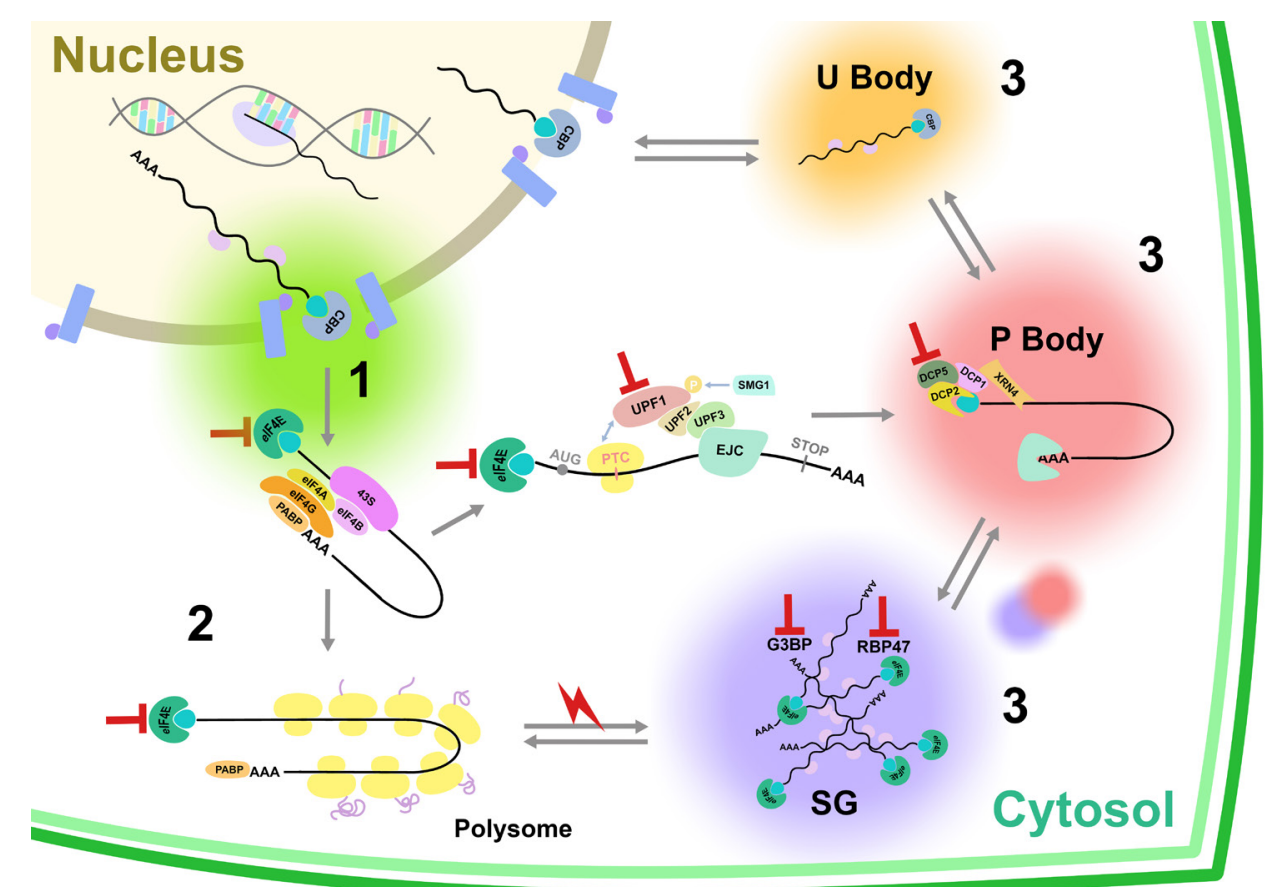

Figure 6.1 Model of capped RNA transport route and processing pathway. See the explanation of the various steps in the main text. 


\section{Why not immediately attack at the start of the road? The Nucleopore and passageway of RNAs from nucleus to cytosol as the first site of cap-snatching}

The nucleopore complex (NPC) is the gate where receptors shuttle various cargo molecules in or out of the nucleus for a number of biological processes. Here, nuclear mRNAs constantly transit and to become appointed to specific cytosolic sites for further downstream processes. For influenza virus, the RdRp interacts with the C-terminal domain of host RNA polymerase II (pol II) to access nascent mRNAs for cap-snatching (Engelhardt et al. 2005). When bunyaviruses indeed would collect capped RNA leaders at NPC, it would somewhat resemble the strategy as applied by influenza virus, i.e. get as close to the access point/start of the flow of capped-RNA molecules as possible. On this point NPCs provide a continuous nuclear efflux of capped RNA and present the first possibility for bunyaviruses to access these capped RNAs. In our observations, both N proteins of TSWV and Rice stripe virus (RSV) show a perinuclear localization, overlapping with RanGAP2, the same factor described above that has a crucial role in the nucleocytoplasmic transport pathway through NCP. Moreover, when transiently expressing the RanGAP2 WPP domain fused to a nuclear localization signal (NLS), portion of the $\mathrm{N}$ protein molecules also show up in the nucleus (Chapter 4 ). This is the first indication that these viruses possibly interact with the RanGAP-mediated branch of the nucleocytoplasmic trafficking pathway. Although speculative, whether this reflects a hitchhike strategy to access nucleocytoplasmic mRNA trafficking needs to be further investigated.

Nucleocytoplasmic transport through NPC is a complicated process. Depending on cargo and receptors, four pathways are distinguished, namely Karyopherin $\alpha / \beta$ transport, transportin transport, exportin transport and bulk mRNA transport (Carmody and Wente 2009; Moore et al. 2020) (Fig. 6.2). The first three types of transport rely on the Ran cycle involving RanGAP, whereas the fourth one is responsible for bulk mRNA transport and relies on ATP-dependent RNA helicase activity of DDX19. The earlier described two classes of capped RNAs (the noncoding snRNAs and the host cytoplasmic mRNAs) rely on different pathways to move from nucleus to cytosol. The major class of RNA pol II transcribed mRNA requires NXF1-NXT1 exportin proteins to enter the nucleopore from the nuclear side and the DDX19 helicase to disassociate from it at the cytoplasmic side (Moore et al. 2020), whereas translation initiation factor elF4E takes over for further release into the cytosol and to become engaged in translation. Besides this role, elF4E also binds some specific mRNAs already in the nucleus, and mediates their export (Borden and Culjkovic-Kraljacic 2010; Topisirovic et al. 2009). 


\section{Chapter 6}

elF4E ribonuclear particles (RNPs) depend on CRM1 for export rather than TAP/NXF1, which is in charge of bulk mRNA export pathway (Culjkovic et al. 2005). Overexpression of elF4E enhanced the export of its binding mRNAs through the elevation of RanBP1 and the RNA export factors, Gle1 and DDX19 (Culjkovic-Kraljacic et al. 2012). The snRNA export through NPC also depends on exportin CMR1, and is driven by RanGTP (Carmody and Wente 2009). RanGTP hydrolysis recycles through RanGAP in the cytosol and RCC1 in the nucleus.

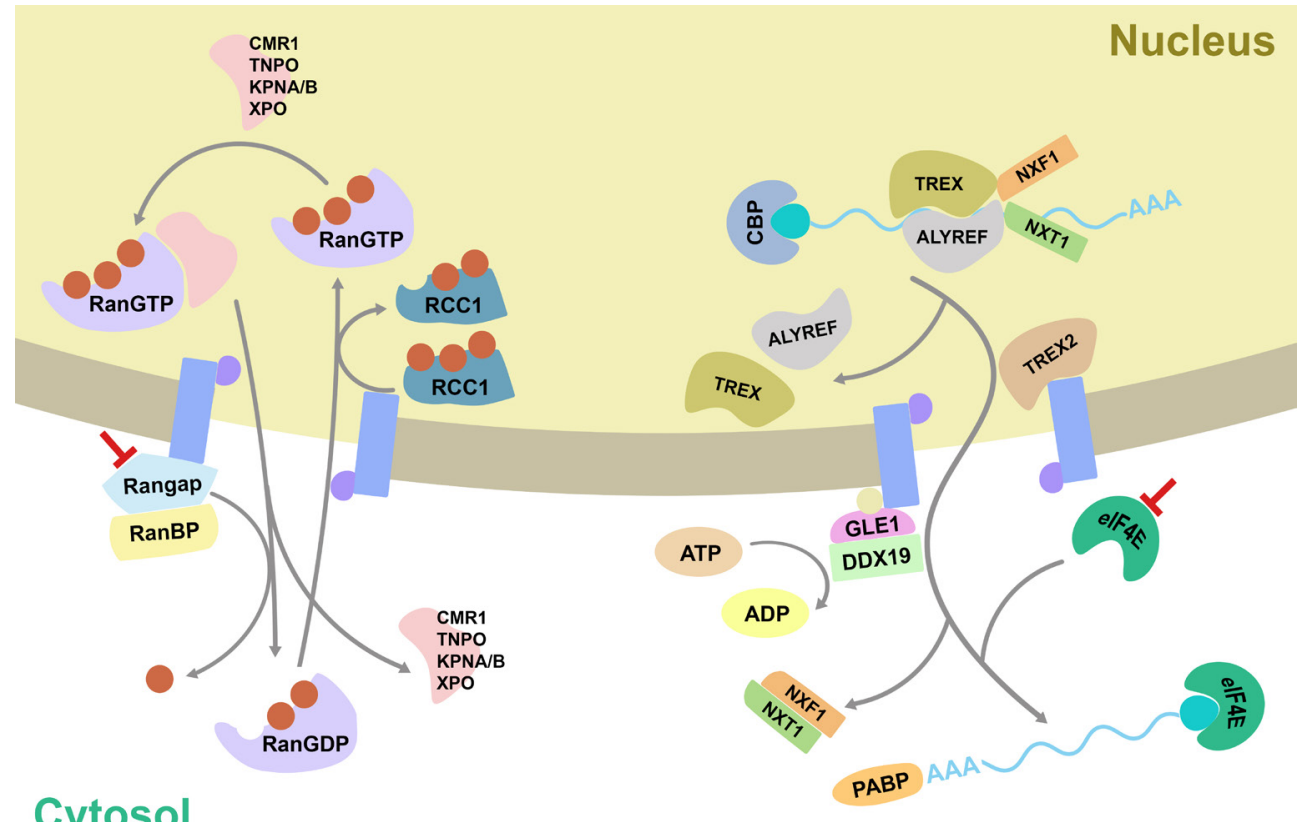

Figure 6.2 Nucleopore export pathways. Left is RanGTP mediated export pathway and right is mRNA export pathway.

When we silenced $\operatorname{RanGAP}(s)$ to interfere with the perinuclear localization of viral N protein, silencing of RanGAP2, only, did not affect the accumulation of TSWV, and viral titres were more or less similar to those from GUS silenced control plants. In contrast, when both RanGAP homologs (1 \& 2) were silenced in plants, TSWV accumulation decreased by $30 \%$. This not only supports earlier findings on a redundancy of RanGAPs in plants (RodrigoPeiris et al. 2011), but also indicates that their depletion directly affects viral replicationtranscription. Considering the function of RanGAP and the possible interaction of TSWV N with Ran WPP (Chapter 4), it is tempting to propose that depletion of RanGAP interferes with the perinuclear localization of N protein/RNPs at NPC, and thereby prevents access to the 
nuclear efflux of capped RNA for viral genome transcription. Whether depletion of RanGAP indirectly affects the bulk mRNA export either positive or negative, was not tested in this study. The observations made after silencing RanGAP, and pointing to a possible role of NPC in viral genome transcription, are supported by observations made on silencing of elF4E. Initially this protein was selected as a target for silencing, being a component of SG (Chapter 4), but this protein is also reported to facilitate nuclear mRNA export by the Ran-GTP driven trafficking pathway (Osborne and Borden 2015). Furthermore, elF4E has also been shown to elevate bulk mRNA export factors Gle1 and the RNA helicase DDX19, indicating a crosstalk between elF4E and the bulk mRNA export pathways, leading to enhanced bulk mRNA export levels (Culjkovic-Kraljacic et al. 2012). The observed increase of TSWV replication in plants silenced on elF4E, might then be explained by an accumulation of bulk mRNAs at the NPC due to lowered levels of DDX19 and due to a depletion of elF4E, to take mRNAs further into the cytosol for translation. The observed, and surprising increase in TSWV replication, after silencing of elF4E, is also interesting knowing that eIF4E plays a major role (in the eIF4G complex) for translation of mRNAs, and indicates that translation of TSWV mRNAs does not appear to be inhibited by depletion of elF4E. This suggests that the virus, like earlier suggested for the SNV hantavirus and Crimean Congo haemorrhagic fever virus (CCHFV) nairovirus (Jeeva et al. 2017a), may have adopted a unique (elF4E-independent) N-proteinmediated translation strategy to enable and stimulate translation of viral mRNAs during their competition with host cellular mRNAs for the translational machinery. This idea is further supported by earlier studies on TSWV that have shown translational enhancement of viral mRNAs by the N and NSs proteins (Geerts-Dimitriadou et al. 2012).

Although more evidence is needed to strengthen the candidacy of NCP as a potential (major?) cap-snatching site, the idea of hijacking the nuclear mRNA export pathway directly at NPC, is quite tempting. On this point it is also of interest to note that for many animal-infecting bunyaviruses, their RNPs, or transiently expressed N proteins, were observed to concentrate in the perinuclear region (Heath et al. 2001; Ravkov and Compans 2001; Reichelt et al. 2004). Whether this perinuclear localization reflects the need to create a viral factory for particle assembly or is required for cap-snatching (or both), or even, as suggested by some, results from sequestration by the host to avoid N/RNPs to go further into replication, still needs to be resolved (Andersson et al. 2004a; Andersson et al. 2004b; Heath et al. 2001). Despite all this, whether and how, mechanistically, capped RNA from these nuclear export pathways are targeted and whether this indeed benefits bunyaviruses, remains unclear. Silencing specific receptors from different RNA export pathways is one of the approaches 
that could be pursued. In addition, the identification of host proteins interacting with the $\mathrm{N}$ proteins of various bunyaviruses could also point towards receptor proteins from mRNA nuclear export pathways.

An interplay with and hitchhike of on nuclear mRNA export pathways would not be unique. Although distinct from the bunyaviruses, the nuclear replicating Influenza viruses, with functional similar RdRps and N proteins, rely on the (RanGAP-dependent) Karyopherin $\alpha / \beta$ transport pathway to reach the nucleus (Swale et al. 2020). Influenza viral mRNAs produced, on the other hand, depend on the cellular NXF1/TAP pathway for export out of the nucleus. Furthermore, although Influenza polymerase units can be found widely distributed in the nucleus, only Influenza replicase/transcriptase complexes collected from the nuclear matrix are suggested to present true replication complexes (Lopez-Turiso et al. 1990). Whether this implies that Influenza viral replication occurs in close proximity of NPC, remains unknown. However, all these findings fit with the idea of RNA polymerase synthesis and modifications to take place in nuclear substructures, for example the nuclear envelop matrix.

\section{Why wait just at the end of the road? Cap-snatching at PB and SG, the cytoplasmic RNA granules near/at the end of the mRNA life cycle}

Cellular RNA granules present a complicated network that connects nuclear and cytoplasmic RNA processing. Previous data have pointed towards cytoplasmic PB as source of host cellular mRNAs for cap-snatching. In studies on the animal-infecting SNV hantavirus Mir et al showed that its $\mathrm{N}$ protein localizes to $\mathrm{P}$ bodies and exhibits cap-binding affinity (Mir et al. 2008b; Mir et al. 2010). Transcripts with PTCs, and ending up at P bodies, also appeared better used than functional transcripts during SNV cap-snatching. However, depletion of $P$ bodies did not lead to reduced viral transcription. Also Rift valley fever virus (RVFV) was observed to snatch capped RNA leaders from Decapping enzyme2 (DCP2)-accessible transcripts (Hopkins et al. 2013). Similar indications have recently been reported for TSWV, and its $\mathrm{N}$ protein observed to (partially) localize to P bodies as well. Knock-out of DCP2 in Arabidopsis resulted in the increase of the TSWV capping rate ( $M a$ et al. 2019). Although these data indicate that $P$ bodies may serve as a source from where bunyaviruses snatch capped-RNA leaders, their major role in RNA decay and being highly enriched in decapping enzymes and nucleases, also poses a risk to the virus from which its mRNAs have to be protected to avoid degradation. As an alternative, it would seem more logical and safer to snatch capped RNA leaders from mRNAs stalled in translation and stored at neighbouring 
SGs. The mRNAs in there, still contain a 5'cap, in contrast to the mRNAs in P bodies of which a part already lacks a 5'cap and are being degraded. However, whether translationally stalled mRNAs in SG are as accessible as the transcripts present in PB can be debated. The observation that SNV hantavirus replicated at normal titres in cells depleted from PB, also led the authors from that study to suggest that the virus might also access host mRNAs from elsewhere, before these reached PB. Furthermore, for several bunyaviral $\mathrm{N}$ proteins affinity to 5' cap-structures was reported, and/or translational enhancement by $\mathrm{N}$ demonstrated (Geerts-Dimitriadou et al. 2012; Jeeva et al. 2017a; Jeeva et al. 2017b; Mir et al. 2010). For this reason, one would also expect these $\mathrm{N}$ proteins to localize/condense at any cytosolic RNA granule enriched on capped-RNA molecules. In situ localization analyses of various (plant- and animal infecting) bunyaviral N proteins, transiently expressed, or during TSWVinfection in planta and SBV infection of animal cells, indeed confirmed this idea (Chapter 4). The results showed that besides PB their N protein also co-localized to SG, and even preferably to SG docked on PB. Even the SNV N protein that was earlier found to only localize in P bodies, in this study localized at PBs and SGs. Bunyaviruses might thus have adopted a (generic) strategy to access not only $P$ bodies but also SGs for cap-snatching. Since $\mathrm{P}$ bodies and SGs are dynamically linked structures and their association is enhanced by viral infection, the use of both as a source for capped-RNA is very plausible. In addition, and not entirely surprising along these lines of thought, the SBV N protein was also observed to co-localize to cytoplasmic maturing $U$ bodies, enriched for capped snRNAs, and found in close proximity to $\mathrm{P}$ bodies, although this was only observed to a minor extent (data not shown). Interestingly, Influenza viral transcripts, although depending on nuclear sources for capped RNA leaders, appeared enriched in the presence of leader sequences from nuclear snRNAs(Gu et al. 2015).

The competition assays with functional GFP and non-functional GFP (nsGFP, containing a PTC) transcripts, that are directed to PB and SG respectively, supported a model for usage of capped-RNA from both PBs and SGs for TSWV cap-snatching (Chapter 5). During earlier studies with SNV (Cheng and Mir 2012; Mir et al. 2008b), capped leader sequences from non-functional GFP, and targeting PBs, were more prevalent in viral mRNAs, compared to those of the functional GFP transcripts. However, during these assays and prior to SNV infection, animal cells were transfected with either the functional GFP construct or nonfunctional GFP-PCT only. During the experiments performed in this thesis, both nsGFP and GFP transcripts were offered simultaneously. In addition, plants were subjected to heat stress for SG induction, for targeting of functional GFP into a translational arrest at SG, prior 
to TSWV infection and cap-snatching. During that experiment the number of capped- leader sequences collected from nsGFP and found in viral mRNAs was slightly lower than from functional GFP. These results show that $P$ bodies do not appear the exclusive source of capped-RNA. The usage of P body-located transcripts is also confirmed by silencing of UPF1. TSWV titres showed a reduction in UPF1-silenced plants, although relatively mild compared to those in GUS-silenced plants. UPF1 is a crucial regulator of the NMD pathway and directs non-functional GFP to P bodies for further turnover (Kim and Maquat 2019). Silencing of UPF1 gene thus cuts off the transport of nonsense RNAs to $P$ bodies and reduces one of the likely cap donor streams. However, a recent study has pointed out that the UPF1 mediated NMD pathway seems to occur in "polysomal-derived" complexes, and not at PBs (Sulkowska et al. 2020). This would then support the role of upstream sources within the translational machinery in providing capped RNAs, a suggestion that was also raised by Mir et al (2008) to explain some of their results. Based on all these results, one might thus debate on the role of $\mathrm{P}$ bodies as the (major/sole) source of capped RNA for cap snatching with the cytoplasmic replicating bunyaviruses. A similar method was used to test the role of SGs in cap-snatching in plants subjected top heat stress to induce SG formation. Analysis of the snatched cappedRNA leader sequences on viral mRNAs revealed the presence of long leader sequences (Chapter 5). As long transcripts tend to go to stress granules upon heat stress (Khong et al. 2017), this altogether suggests that the virus is able to use transcripts that enter SGs. However, the exact localization of these produced transcripts needs to be confirmed, before being able to conclude whether P body and/or SG are indeed being used for cap-snatching.

Although P bodies and SGs both may serve as potential cytoplasmic pools of cellular mRNAs for cap-snatching, they are involved in various functions and act pro- or antiviral for many viruses (Malinowska et al. 2016) (Chapter 2), and this seems to apply to TSWV and SBV as well (Chapter 4). When key elements of either P bodies or SGs were silenced, titres for TSWV/SBV went up, also suggesting a putative role in host defence against these viruses. Interestingly, when PB and SG elements were both silenced, viral titres did not go up to combined titre levels obtained from their individual silencing. Although the potential role of RNA granules in cap-snatching has been explored, due to their complex functions in RNA processing, transient existence (SGs), their intimacy and exchange of elements, it is very plausible that these RNA granules are just complementing to other (primary) sites of cap-snatching, facilitating the virus to maintain replication rates when access to upstream sources of capped-RNA are blocked/inhibited. 
Although bunyaviral N proteins co-localize at PB and SGs, reflecting their affinity for 5'capstructures, and possibly a generic feature for all bunyaviral $\mathrm{N}$ proteins, the fact that these RNA condensates are relatively large and can be easily seen, also raises the question whether or not this has blinded us so far from a more complex cap-snatching process taking place more upstream, and at less visible spots. After all, and in the end, all cytoplasmic mRNAs, even those just bound by viral $\mathrm{N}$ protein at the $5^{\prime}$ cap, end up and concentrate at PBs for their turn-over. Current evidence as presented in this thesis research at least indicates that there is more to the story of cap snatching than just PBs. Considering that cap-snatching requires the endonuclease activity of the viral $\mathrm{RdRp}$, co-localization studies of $\mathrm{N}$ protein with the viral RdRp will be one of the next major tasks needed to possibly solve the enigma on the cytoplasmic pools of mRNA needed for cap-snatching, as will further be explained in the next section.

\section{Lead the way? Host proteins interacting with bunyaviral $\mathbf{N}$ proteins to localize at and access leader sequences of host cellular mRNA for cap- snatching}

Cap-snatching is a process that involves several activities performed by a few interacting proteins. The $L(R d R p)$ protein is a major player because it contains some essential functions required for this process: 1 ) endonuclease activity (within the $\mathrm{N}$-terminus) to cleave cappedRNA leaders from host cellular mRNAs, 2) RNA polymerase activity (within a central domain) and 3) a cap-binding domain (within the C-terminus). However, the cap-binding domain in the $L$ protein still remains putative and mysterious among bunyaviruses. Structural folding analysis has shown some resemblance to the cap-binding domain (CBD) within the Influenza PB2 protein, but in the case of a reptarena virus RdRp protein, this domain appeared nonfunctional(Rosenthal et al. 2017). In case of the California Academy of Sciences virus (CASV) and RVFV L proteins, the affinity to biochemically produced cap structures appeared not as strong as for 5 'cap binding translation initiation factors(Olschewski et al. 2020). Considering that both $\mathrm{N}$ and $\mathrm{L}$ have earlier been demonstrated in bunyavirus reverse genetics systems to be required for replication-transcription(Cheng et al. 2014), it is very likely that they intimately interact and are both essential to enable cap-snatching. To compensate for the observed lower affinity of the L CBD for $5^{\prime}$ caps, a concerted action with the $\mathrm{N}$ protein to strongly bind 5'cap structures has been an appealing idea. As major component of the viral RNPs, $\mathrm{N}$ protein is important in many viral processes, and some studies do provide support for a possible role in cap-snatching. The SNV N protein exhibits a higher affinity to 
capped RNA when being offered a 1:1 mix of capped and uncapped RNA (Mir et al. 2010). For the Junin, Tacaribe, and Pichinde arenaviruses, their $\mathrm{N}$ protein has been shown to be able to interact with 7-methyl-guanosine (=cap) (Jeeva et al. 2017a; Jeeva et al. 2017b). For other viruses, for example, Crimean-congo haemorrhagic fever nairovirus (CCHFV) and the TSWV tospovirus, their $\mathrm{N}$ protein has been shown to enhance translation of mRNAs (Geerts-Dimitriadou et al. 2012; Jeeva et al. 2017a; Jeeva et al. 2017b). In a few studies on SNV and CCHFV, and in this thesis demonstrated for TSWV, viral replication-transcription was not affected by depletion of elF4E, and indicated a role of $\mathrm{N}$ as surrogate of elF4E. The exact role of $\mathrm{N}$ protein in cap-snatching and how it helps $\mathrm{L}$ protein to perform capsnatching still remains unsolved. Furthermore, the role of additional host cellular factors in this process, recruited by either the $\mathrm{N}$ or L protein, cannot be excluded. After all, some of these factors might help to direct and provide access to the source of host cellular mRNA. On this point, only limited information is available. For hantavirus, unknown host factors have been suggested to play a key role in cap-snatching (Jeeva et al. 2019). A few studies have identified some host proteins interacting with $\mathrm{N}$ protein but for several the exact role has still to be resolved. For example, the cellular protein MOV10 has been shown to interact with $\mathrm{N}$ protein of Severe fever with thrombocytopenia syndrome virus (SFTSV) and observed to restrict the assembly of RNPs (Mo et al. 2020). Hantavirus N protein interacts with the apoptosis enhancer $=$ Daxx and the predicted binding site is related to nuclear localization (Li et al. 2002b), suggesting $\mathrm{N}$ protein plays a role in liming apoptosis. In relation to the idea that the NPC might present a site for cap-snatching, it is interesting to note that hantavirus $\mathrm{N}$ protein also shows interaction with importina, which is responsible for shuttling nuclear factor kappa $B$ to the nucleus, thus interfering the nuclear transport pathway (Taylor et al. 2009). Hantavirus $N$ protein was also found to interact with Small Ubiquitin-related MOdifier-1 (SUMO-1) and SUMO-1 conjugating enzyme (ubc9) proteins and this interaction was crucial for perinuclear localization of $N$ protein (Maeda et al. 2003). However, still more work is needed to identify the interactome of $\mathrm{N}$ (and $\mathrm{L}$ ) and analyse the functional relevance of host proteins for cap-snatching.

A direct first view on possible cellular foci involved in viral transcription-replication processes may be obtained by in situ localization of essential (non)structural proteins, as many viruses establish a viral factory that often relies on (and hijacks) host cellular structures(Hyodo and Okuno 2020). The localization of the N protein is a good indicator for the cytoplasmicreplicating NSVs in cap-snatching, and the first glimpse of this has been obtained with SNV, of which the $\mathrm{N}$ protein exclusively appeared to localize at $\mathrm{P}$ bodies and showed a preferential 
binding to capped RNAs (Mir et al. 2008b; Mir et al. 2010). In light of cellular sites being of potential importance for bunyavirus cap-snatching, like the NPC with a continuous capped RNA nuclear efflux as described in this thesis (Chapter 4), and most likely being conserved/ generic for all cytoplasmic bunyviruses, an overview was made on the reported localization of bunyavirus $\mathrm{N}$ protein (Table 6.1 ). Whereas the SNV N protein initially was reported to

Table 6.1 Localization of several bunyavirus $\mathrm{N}$ proteins.

\begin{tabular}{|c|c|c|c|}
\hline Genus & Species & Specific location & References \\
\hline Hantaviridae & SNV, BCCV & P body, SG, perinuclear and Golgi & $\begin{array}{c}\text { (Mir et al. 2008b), } \\
\text { (Ravkov and Compans } \\
\text { 2001) }\end{array}$ \\
\hline Phenuiviridae & RVFV, RSV & P body, SG, perinuclear & $\begin{array}{c}\text { (Hopkins et al. 2013), } \\
\text { chapter } 4\end{array}$ \\
\hline Arenaviridae & TCRV & Cytosolic, punctate & (Baird et al. 2012) \\
\hline Tospoviridae & IYSV, CaCV, TSWV & P body, SG, perinuclear, ER & $\begin{array}{c}\text { (Tripathi et al. 2015) } \\
\text { (Widana Gamage } \\
\text { and Dietzgen 2017), } \\
\text { chapter } 4\end{array}$ \\
\hline Peribunyaviridae & LACV & Perinuclear and Golgi & $\begin{array}{l}\text { (Reichelt et al. } \\
\text { 2004) }\end{array}$ \\
\hline Narioviridae & CCHFV & P body, SG, perinuclear and Golgi & $\begin{array}{l}\text { (Andersson et al. } \\
\text { 2004a; Andersson et } \\
\text { al. 2004b), chapter } 4\end{array}$ \\
\hline
\end{tabular}

localize at P bodies only (Mir et al. 2008b), in this study it also co-localized to SG (Chapter 4). RVFV N protein colocalizes with a P body resident protein (Hopkins et al. 2013). Tacaribe arenavirus $\mathrm{N}$ protein induces cytosolic punctates and localizes together with viral RNAs(Baird et al. 2012). Both plant-infecting Iris yellow spot virus (IYSV) and Capsicum chlorosis virus (CaCV) tospovirus N proteins localize at perinuclear sites (Tripathi et al. 2015; Widana Gamage and Dietzgen 2017), and a similar localization was observed for TSWV and RSV N proteins in this thesis. Notably, a perinuclear localization of $\mathrm{N}$ protein in mammalian cells has also been reported for several animal infecting bunyaviral RNPs/N proteins (Andersson et al. 2004a; Andersson et al. 2004b; Ravkov and Compans 2001; Reichelt et al. 2004), including several hantavirus $\mathrm{N}$ proteins. In the latter cases some were reported to occur at late stages of viral infection (Ramanathan et al. 2007). The TSWV N protein showed a perinuclear localization in both animal and plant cells. In animal cells the trafficking of $\mathrm{N}$ to the perinuclear region depended on microtubules (Snippe et al. 2005), whereas in plants 
cells, $\mathrm{N}$ dispersed over ER and cytosol and its movement depended on actin (Ribeiro et al. 2008). Although it still remains unclear why/how $N$ proteins depend on different host factors for their intracellular movement, it is very well possible that part of the variations observed, are due to differences in the cellular architecture (plant versus animal) that required adaptation of the virus. However, seeing that most of the $\mathrm{N}$ proteins have been reported to also localize perinuclear hints at a possible role for the perinuclear region in viral replication as well. Whether the perinuclear localization is to create a viral factory for particle assembly or for cap-snatching, or instead, results from host sequestration to avoid N/RNPs to go further into replication as also proposed by some authors, still requires further investigation (Andersson et al. 2004a; Andersson et al. 2004b; Heath et al. 2001).

Even with the possibility of bunyavirus $\mathrm{N}$ proteins having a major role in cap-snatching, without the $L$ protein there will not be any endonuclease cleavage of capped RNA leaders. For Influenza virus the binding of the polymerase complex (PA-PB1-PB2) to the CTD of RNA pol II has pointed towards RNA pol II transcription foci as the site for Influenza transcription (Engelhardt et al. 2005), supported by the finding that inhibitors of RNA pol II directly inhibited viral transcription/replication. On the other hand, influenza viral N protein was also observed to localize to PBs in the presence of the NS1 protein, but localized to SG in the absence of this protein (Mok et al. 2012). Although Influenza viruses and bunyaviruses are very distinct in many ways, these observations may as well be considered as support for the idea that the co-localization of bunyavirus $\mathrm{N}$ to PBs and SG relate to repression of their antiviral activity. To unravel the process for the cytoplasmic-replicating bunyaviruses, and further indicate the relevance of PBs, SG, NPC or any other cytosolic source in cap-snatching, studies need to be performed on the localization of bunyaviral L proteins. Due to the size ( 250-450 kDa) and (low) expression levels of these $L$ proteins, determining their subcellular localization and the identification of interacting host proteins, are major challenges. Attempts to transiently express and localize the TSWV L protein so far have failed.

\section{Side effects on the way: down-regulation of photosynthetic genes expression}

By bioinformatic analysis, non-viral cap leader sequences used by TSWV were analysed and the originating host cellular donor transcripts identified (Chapter 5). Hundreds of donor transcripts were mapped to different biological pathways, but there was a significant abundance of transcripts that mapped to genes related to the photosynthesis pathway. A look at gene expression levels in non-infected plants shows that those involved in 
photosynthesis are (usually) highly expressed. This makes them more likely candidate transcripts to be encountered by the virus on the cytoplasmic RNA trafficking route and subsequently being used for cap-snatching. This is also in agreement with transcriptome data from TSWV-infected Arabidopsis where a clear downregulation in the expression of genes from the photosynthesis pathway is observed (Chapter 3). Many plant viruses have been reported to cause chlorosis and/or yellowing symptoms on host plants via changing the photosynthetic pathway through many different ways (Zhao et al. 2016). The data collected in this study supports the idea that cap-snatching of gene transcripts from the photosynthesis pathway leads to their translational repression and contributes to disease symptomatology like chlorosis. This not only limits to TSWV, but likely applies to all plantinfecting NSVs, i.e. the tenuiviruses and emaraviruses as well. 


\section{Concluding remarks}

The research described in this thesis investigated the cap-snatching mechanism of cytoplasmic replicating NSVs with emphasis on the identification of the cytoplasmic source(s) of host cellular mRNAs used as cap donor. Whereas some earlier reports have pointed towards $P$ bodies, the results described in this study point to SG and the NPC as potential source/foci as well. Support for this is being provided by in situ localization analysis of $\mathrm{N}$ proteins from different (plant and animal infecting) NSVs to these sites, the effect of silencing of host components related to these sites on viral replication, and the observation that capped-RNA leader sequences from functional and non-functional (NMD-destined) transcripts both are being used well during cap-snatching when offered simultaneously. Despite the progress made, it still remains elusive which cytoplasmic condensation/foci present are the predominant/sole source of cap donors, and how these viruses get access to these sources. The observation of a potential interaction between TSWV N and RanGAP, enabling TSWV N to access the NPC, tempts us to speculate on the NPC presenting a major site from where capped RNA sequences can be selected. It makes sense from the point of view that NPC offers a constant and concentrated flow of nuclear mRNA to the cytosol. When the mRNAs are bound there by N protein at the 5' cap, mRNAs may then still continue on their road to engage in (elF4E-independent) translation, or further downstream to end up and concentrate at SG, PB or "polysomal-derived" complexes for NMD-transcripts. Whereas SG and PB condensations are easily discerned and bunyavirus $\mathrm{N}$ proteins are generally being produced in relative high amounts, their co-localization could thus be misleading, and the results of this study has indicated that we need to be more cautious. Although it is possible that any source downstream NPC, like PB and SG, may play a complementary role in providing cap donor RNAs, only a co-localization with $\mathrm{N}$ and the viral RdRp protein at any of these foci will reveal where capped-RNA leader sequences are truly cleaved from host cellular mRNAs. This now presents one of the first and major challenges to address in order to advance. During these studies, identified host proteins that lead the viral $N$ and $L$ proteins to these capped RNA sequences will become promising target(s) for future virus control. 


\title{
Appendix
}

\author{
Reference list \\ Summary \\ Acknowledgements \\ About the author \\ Publication list
}

Education statement 


\section{Reference list}

\section{A}

Abudurexiti, A., Adkins, S., Alioto, D., Alkhovsky, S. V., Avsic-Zupanc, T., Ballinger, M. J., Bente, D. A., Beer, M., Bergeron, E., Blair, C. D., Briese, T., Buchmeier, M. J., Burt, F. J., Calisher, C. H., Chang, C., Charrel, R. N., Choi, I. R., Clegg, J. C. S., de la Torre, J. C., de Lamballerie, X., Deng, F., Di Serio, F., Digiaro, M., Drebot, M. A., Duan, X., Ebihara, H., Elbeaino, T., Ergunay, K., Fulhorst, C. F., Garrison, A. R., Gao, G. F., Gonzalez, J. J., Groschup, M. H., Gunther, S., Haenni, A. L., Hall, R. A., Hepojoki, J., Hewson, R., Hu, Z., Hughes, H. R., Jonson, M. G., Junglen, S., Klempa, B., Klingstrom, J., Kou, C., Laenen, L., Lambert, A. J., Langevin, S. A., Liu, D., Lukashevich, I. S., Luo, T., Lu, C., Maes, P., de Souza, W. M., Marklewitz, M., Martelli, G. P., Matsuno, K., Mielke-Ehret, N., Minutolo, M., Mirazimi, A., Moming, A., Muhlbach, H. P., Naidu, R., Navarro, B., Nunes, M. R. T., Palacios, G., Papa, A., Pauvolid-Correa, A., Paweska, J. T., Qiao, J., Radoshitzky, S. R., Resende, R. O., Romanowski, V., Sall, A. A., Salvato, M. S., Sasaya, T., Shen, S., Shi, X., Shirako, Y., Simmonds, P., Sironi, M., Song, J. W., Spengler, J. R., Stenglein, M. D., Su, Z., Sun, S., Tang, S., Turina, M., Wang, B., Wang, C., Wang, H., Wang, J., Wei, T., Whitfield, A. E., Zerbini, F. M., Zhang, J., Zhang, L., Zhang, Y., Zhang, Y. Z., Zhang, Y., Zhou, X., Zhu, L., and Kuhn, J. H. 2019. Taxonomy of the order Bunyavirales: update 2019. Archives of virology 164:1949-1965.

Ahlquist, P., Noueiry, A. O., Lee, W. M., Kushner, D. B., and Dye, B. T. 2003. Host factors in positivestrand RNA virus genome replication. J Virol 77:8181-8186.

Anders, S., Pyl, P. T., and Huber, W. 2015. HTSeq-a Python framework to work with high-throughput sequencing data. Bioinformatics 31:166-169.

Anderson, P., and Kedersha, N. 2008. Stress granules: the Tao of RNA triage. Trends Biochem Sci 33:141-150.

Anderson, P., and Kedersha, N. 2009. RNA granules: post-transcriptional and epigenetic modulators of gene expression. Nat. Rev. Mol. Cell Biol. 10:430-436.

Anderson, P., Kedersha, N., and Ivanov, P. 2015. Stress granules, P bodies and cancer. BBA-GENE REGUL MECH 1849:861-870.

Andersson, I., Bladh, L., Mousavi-Jazi, M., Magnusson, K. E., Lundkvist, A., Haller, O., and Mirazimi, A. 2004a. Human MxA protein inhibits the replication of Crimean-Congo hemorrhagic fever virus. J Virol 78:4323-4329.

Andersson, I., Simon, M., Lundkvist, A., Nilsson, M., Holmström, A., Elgh, F., and Mirazimi, A. 2004b. Role of actin filaments in targeting of Crimean Congo hemorrhagic fever virus nucleocapsid protein to perinuclear regions of mammalian cells. J Med Virol 72:83-93.

Azevedo, J., Garcia, D., Pontier, D., Ohnesorge, S., Yu, A., Garcia, S., Braun, L., Bergdoll, M., Hakimi, M. A., Lagrange, T., and Voinnet, O. 2010. Argonaute quenching and global changes in Dicer homeostasis caused by a pathogen-encoded GW repeat protein. Genes Dev 24:904-915.

\section{B}

Bailey, T. L. 2020. STREME: Accurate and versatile sequence motif discovery. Biorxiv https://doi. org/10.1101/2020.11.23.394619

Baird, N. L., York, J., and Nunberg, J. H. 2012. Arenavirus infection induces discrete cytosolic structures for RNA replication. J Virol 86:11301-11310.

Barr, J. N. 2007. Bunyavirus mRNA synthesis is coupled to translation to prevent premature transcription termination. Rna 13:731-736.

Baumberger, N., Tsai, C. H., Lie, M., Havecker, E., and Baulcombe, D. C. 2007. The polerovirus silencing suppressor P0 targets Argonaute proteins for degradation. Curr Biol 17:1609-1614. 
Beauchemin, C., Boutet, N., and Laliberte, J. F. 2007. Visualization of the interaction between the precursors of $\mathrm{VPg}$, the viral protein linked to the genome of turnip mosaic virus, and the translation eukaryotic initiation factor iso 4E in Planta. J Virol 81:775-782.

Beaudoin, S., Vanderperre, B., Grenier, C., Tremblay, I., Leduc, F., and Roucou, X. 2009. A large ribonucleoprotein particle induced by cytoplasmic PrP shares striking similarities with the chromatoid body, an RNA granule predicted to function in posttranscriptional gene regulation. Biochimica et Biophysica Acta (BBA) - Mol Cell Res 1793:335-345.

Beckham, C. J., and Parker, R. 2008. P bodies, stress granules, and viral life cycles. Cell Host \& Microbe 3:206-212.

Beckham, C. J., Light, H. R., Amar Nissan, T., Ahlquist, P., Parker, R., and Noueiry, A. 2007. Interactions between brome mosaic virus rnas and cytoplasmic processing bodies. J Virol 81:9759-9768.

Behm-Ansmant, I., Rehwinkel, J., Doerks, T., Stark, A., Bork, P., and Izaurralde, E. 2006. mRNA degradation by miRNAs and GW182 requires both CCR4:NOT deadenylase and DCP1:DCP2 decapping complexes. Genes Dev 20:1885-1898.

Bhasin, H., and Hulskamp, M. 2017. ANGUSTIFOLIA, a plant homolog of CtBP/BARS localizes to stress granules and regulates their formation. Front Plant Sci 8:1004.

Bhullar, D. S., Sheahan, M. B., and Rose, R. J. 2017. RNA processing body (P body) dynamics in mesophyll protoplasts re-initiating cell division. Protoplasma 254:1627-1637.

Bies-Etheve, N., Pontier, D., Lahmy, S., Picart, C., Vega, D., Cooke, R., and Lagrange, T. 2009. RNAdirected DNA methylation requires an AGO4-interacting member of the SPT5 elongation factor family. EMBO Reports 10:649-654.

Bond, C. S., and Fox, A. H. 2009. Paraspeckles: nuclear bodies built on long noncoding RNA. J Cell Biol 186:637-644.

Borden, K. L., and Culjkovic-Kraljacic, B. 2010. Ribavirin as an anti-cancer therapy: acute myeloid leukemia and beyond? Leukemia \& lymphoma 51:1805-1815.

Bortolamiol, D., Pazhouhandeh, M., Marrocco, K., Genschik, P., and Ziegler-Graff, V. 2007. The polerovirus F box protein P0 targets Argonaute1 to suppress RNA silencing. Curr Biol 17:1615-1621.

Boudonck, K., Dolan, L., and Shaw, P. J. 1998. Coiled body numbers in the Arabidopsis root epidermis are regulated by cell type, developmental stage and cell cycle parameters. J Cell Sci 111 ( Pt 24):3687-3694.

Boudonck, K., Dolan, L., and Shaw, P. J. 1999. The movement of coiled bodies visualized in living plant cells by the green fluorescent protein. Mol Biol Cell 10:2297-2307.

Boulon, S., Westman, B. J., Hutten, S., Boisvert, F. M., and Lamond, A. I. 2010. The Nucleolus under Stress. Mol Cell 40:216-227.

Braun, J. E., Huntzinger, E., and Izaurralde, E. 2013. The role of GW182 proteins in miRNA-mediated gene silencing. Adv. Exp Med Biol 768:147-163.

Brengues, M. 2005. Movement of eukaryotic mRNAs between polysomes and cytoplasmic processing bodies. Science 310:486-489.

Brengues, M., Parker, R., and Fox, T. 2007. Accumulation of polyadenylated mRNA, Pab1p, elF4E, and elF4G with P bodies in Saccharomyces cerevisiae. Mol Biol Cell 18:2592-2602.

Brodersen, P., Sakvarelidze-Achard, L., Bruun-Rasmussen, M., Dunoyer, P., Yamamoto, Y. Y., Sieburth, L., and Voinnet, O. 2008. Widespread translational inhibition by plant miRNAs and siRNAs. Science 320:1185-1190.

Burgui, I., Yanguez, E., Sonenberg, N., and Nieto, A. 2007. Influenza virus mRNA translation revisited: is the elF4E cap-binding factor required for viral mRNA translation? J Virol 81:12427-12438.

Butterbach, P., Verlaan, M. G., Dullemans, A., Lohuis, D., Visser, R. G., Bai, Y., and Kormelink, R. 2014. Tomato yellow leaf curl virus resistance by Ty-1 involves increased cytosine methylation of viral genomes and is compromised by Cucumber mosaic virus infection. Proc Natl Acad Sci USA 111:12942-12947. 


\section{C}

Camacho, C., Coulouris, G., Avagyan, V., Ma, N., Papadopoulos, J., Bealer, K., and Madden, T. L. 2009. BLAST+: architecture and applications. BMC bioinformatics 10:421.

Canetta, E., Kim, S. H., Kalinina, N. O., Shaw, J., Adya, A. K., Gillespie, T., Brown, J. W., and Taliansky, M. 2008. A plant virus movement protein forms ringlike complexes with the major nucleolar protein, fibrillarin, in vitro. J Mol Biol 376:932-937.

Canto, T., Uhrig, J. F., Swanson, M., Wright, K. M., and MacFarlane, S. A. 2006. Translocation of Tomato bushy stunt virus $\mathrm{P} 19$ protein into the nucleus by ALY proteins compromises its silencing suppressor activity. J Virol 80:9064-9072.

Carmody, S. R., and Wente, S. R. 2009. mRNA nuclear export at a glance. J Cell Sci 122:1933-1937.

Chan, A. Y., Vreede, F. T., Smith, M., Engelhardt, O. G., and Fodor, E. 2006. Influenza virus inhibits RNA polymerase II elongation. Virology 351:210-217.

Chang, C. H., Hsu, F. C., Lee, S. C., Lo, Y. S., Wang, J. D., Shaw, J., Taliansky, M., Chang, B. Y., Hsu, Y. H., and Lin, N. S. 2016. The nucleolar fibrillarin protein is required for helper virus-independent longdistance trafficking of a subviral satellite RNA in plants. Plant Cell 28:2586-2602.

Chang, C. K., Chen, C. J., Wu, C. C., Chen, S. W., Shih, S. R., and Kuo, R. L. 2017. Cellular hnRNP A2/B1 interacts with the NP of influenza A virus and impacts viral replication. PLoS One 12:e0188214.

Chantarachot, T., and Bailey-Serres, J. 2018. Polysomes, stress granules, and processing bodies: a dynamic triumvirate controlling cytoplasmic mRNA fate and function. Plant Physiol 176:254-269.

Chekulaeva, M., Mathys, H., Zipprich, J. T., Attig, J., Colic, M., Parker, R., and Filipowicz, W. 2011. miRNA repression involves GW182-mediated recruitment of CCR4-NOT through conserved W-containing motifs. Nat Struct Mol Biol 18:1218-1226.

Cheng, C. P., Jaag, H. M., Jonczyk, M., Serviene, E., and Nagy, P. D. 2007. Expression of the Arabidopsis Xrn4p 5'-3' exoribonuclease facilitates degradation of tombusvirus RNA and promotes rapid emergence of viral variants in plants. Virology 368:238-248.

Cheng, E., and Mir, M. A. 2012. Signatures of host mRNA 5' terminus for efficient hantavirus cap snatching. J Virol 86:10173-10185.

Cheng, E., Wang, Z., and Mir, M. A. 2014. Interaction between hantavirus nucleocapsid protein (N) and RNA-dependent RNA polymerase (RdRp) mutants reveals the requirement of an N-RdRp interaction for viral RNA synthesis. J Virol 88:8706-8712.

Chicois, C., Scheer, H., Garcia, S., Zuber, H., Mutterer, J., Chicher, J., Hammann, P., Gagliardi, D., and Garcia, D. 2018. The UPF1 interactome reveals interaction networks between RNA degradation and translation repression factors in Arabidopsis. Plant J 96:119-132.

Chiu, M. H., Chen, I. H., Baulcombe, D. C., and Tsai, C. H. 2010. The silencing suppressor P25 of Potato virus $\mathrm{X}$ interacts with Argonaute1 and mediates its degradation through the proteasome pathway. Mol Plant Pathol 11:641-649.

Choudury, S. G., Shahid, S., Cuerda-Gil, D., Panda, K., Cullen, A., Ashraf, Q., Sigman, M. J., McCue, A. D., and Slotkin, R. K. 2019. The RNA export factor ALY1 enables genome-wide RNA-directed DNA methylation. Plant Cell 31:759-774.

Cock, P. J., Gruning, B. A., Paszkiewicz, K., and Pritchard, L. 2013. Galaxy tools and workflows for sequence analysis with applications in molecular plant pathology. PeerJ 1:e167.

Coller, J., and Parker, R. 2005. General translational repression by activators of mRNA decapping. Cell 122:875-886.

Collier, S., Pendle, A., Boudonck, K., van Rij, T., Dolan, L., and Shaw, P. 2006. A distant coilin homologue is required for the formation of Cajal bodies in Arabidopsis. Mol Biol Cell 17:2942-2951.

Coursey, T., Regedanz, E., and Bisaro, D. M. 2018. Arabidopsis RNA polymerase V mediates enhanced compaction and silencing of geminivirus and transposon chromatin during host recovery from infection. J Virol 92.7. 
Culjkovic, B., Topisirovic, I., Skrabanek, L., Ruiz-Gutierrez, M., and Borden, K. L. 2005. elF4E promotes nuclear export of cyclin D1 mRNAs via an element in the 3'UTR. J Cell Biol 169:245-256.

Culjkovic-Kraljacic, B., Baguet, A., Volpon, L., Amri, A., and Borden, K. L. 2012. The oncogene elF4E reprograms the nuclear pore complex to promote mRNA export and oncogenic transformation. Cell Rep 2:207-215.

Danecek, P., Bonfield, J. K., Liddle, J., Marshall, J., Ohan, V., Pollard, M. O., Whitwham, A., Keane, T., McCarthy, S. A., Davies, R. M., and Li, H. 2021. Twelve years of SAMtools and BCFtools. GigaScience 10.

Dardick, C. 2007. Comparative expression profiling of Nicotiana benthamiana leaves systemically infected with three fruit tree viruses. Mol Plant Microbe Interact 20:1004-1017.

Decroly, E., Ferron, F., Lescar, J., and Canard, B. 2011. Conventional and unconventional mechanisms for capping viral mRNA. Nat Rev Microbiol 10:51-65.

del Olmo, I., Lopez, J. A., Vazquez, J., Raynaud, C., Pineiro, M., and Jarillo, J. A. 2016. Arabidopsis DNA polymerase epsilon recruits components of Polycomb repressor complex to mediate epigenetic gene silencing. Nucleic Acids Research 44:5597-5614.

Dinh, P. X., Das, A., Franco, R., and Pattnaik, A. K. 2013. Heterogeneous nuclear ribonucleoprotein $\mathrm{K}$ supports vesicular stomatitis virus replication by regulating cell survival and cellular gene expression. J Virol 87:10059-10069.

Dong, O. X., Meteignier, L. V., Plourde, M. B., Ahmed, B., Wang, M., Jensen, C., Jin, H., Moffett, P., Li, X., and Germain, H. 2016. Arabidopsis TAF15b localizes to RNA processing bodies and contributes to snc1-mediated autoimmunity. Mol Plant Microbe Interact 29:247-257.

Dreher, T. W. 2004. Turnip yellow mosaic virus: transfer RNA mimicry, chloroplasts and a C-rich genome. Mol Plant Pathol 5:367-375.

Du, Z. G., Xiao, D. L., Wu, J. G., Jia, D. S., Yuan, Z. J., Liu, Y., Hu, L. Y., Han, Z., Wei, T. Y., Lin, Q. Y., Wu, Z. J., and Xie, L. H. 2011. p2 of Rice stripe virus (RSV) interacts with OsSGS3 and is a silencing suppressor. Mol Plant Pathol 12:808-814.

Du, Z. Y., Chen, A. Z., Chen, W. H., Liao, Q. S., Zhang, H. M., Bao, Y. M., Roossinck, M. J., and Carr, J. P. 2014. Nuclear-cytoplasmic partitioning of Cucumber mosaic virus protein $2 \mathrm{~b}$ determines the balance between its roles as a virulence determinant and an RNA-silencing suppressor. J Virol 88:5228-5241.

Dubois, M.-L., and Boisvert, F.-M. 2016. The nucleolus: structure and function. Springer Int Publishing $\mathrm{Ag}$, Gewerbestrasse 11, Cham, Ch-6330, Switzerland.

Duijsings, D., Kormelink, R., and Goldbach, R. 1999. Alfalfa mosaic virus RNAs serve as cap donors for Tomato spotted wilt virus transcription during coinfection of Nicotiana benthamiana. J Virol 73:5172-5175.

Duijsings, D., Kormelink, R., and Goldbach, R. 2001. In vivo analysis of the TSWV cap-snatching mechanism: single base complementarity and primer length requirements. EMBO J 20:2545-2552.

Dundr, M., and Misteli, T. 2010. Biogenesis of nuclear bodies. Cold Spring Harb Perspect Biol 2:a000711.

\section{$E$}

Engelhardt, O. G., Smith, M., and Fodor, E. 2005. Association of the influenza A virus RNA-dependent RNA polymerase with cellular RNA polymerase II. J Virol 79:5812-5818.

Eulalio, A., Behm-Ansmant, I., and Izaurralde, E. 2007. P bodies: at the crossroads of post-transcriptional pathways. Nat Rev Mol Cell Biol 8:9-22.

Eulalio, A., Huntzinger, E., and Izaurralde, E. 2008. Getting to the root of miRNA-mediated gene silencing. Cell 132:9-14. 
Eulalio, A., Huntzinger, E., Nishihara, T., Rehwinkel, J., Fauser, M., and Izaurralde, E. 2009. Deadenylation is a widespread effect of miRNA regulation. Rna 15:21-32.

Eystathioy, T., Chan, E. K., Tenenbaum, S. A., Keene, J. D., Griffith, K., and Fritzler, M. J. 2002. A phosphorylated cytoplasmic autoantigen, GW182, associates with a unique population of human mRNAs within novel cytoplasmic speckles. Mol Biol Cell 13:1338-1351.

Eystathioy, T., Jakymiw, A., Chan, E. K. L., Seraphin, B., Cougot, N., and Fritzler, M. J. 2003. The GW182 protein colocalizes with mRNA degradation associated proteins hDcp1 and hLSm4 in cytoplasmic GW bodies. Rna-a Publication of the Rna Society 9:1171-1173.

\section{$\mathbf{F}$}

Fang, Y., and Spector, D. L. 2007. Identification of nuclear dicing bodies containing proteins for microrna biogenesis in living Arabidopsis plants. Current Biology 17:818-823.

Fang, Y. Y., Zhao, J. H., Liu, S. W., Wang, S., Duan, C. G., and Guo, H. S. 2016. CMV2b-AGO Interaction is required for the suppression of RDR-dependent antiviral silencing in Arabidopsis. Front Microbiol 7. 1329 .

Feng, M., Cheng, R., Chen, M., Guo, R., Li, L., Feng, Z., Wu, J., Xie, L., Hong, J., Zhang, Z., Kormelink, R., and Tao, X. 2020. Rescue of Tomato spotted wilt virus entirely from complementary DNA clones. Proc Natl Acad Sci USA 117:1181-1190.

Feng, Q., Jagannathan, S., and Bradley, R. K. 2017. The RNA surveillance factor UPF1 represses myogenesis via its E3 ubiquitin ligase activity. Mol Cell 67:239-251.

Fontaine, K. A., Leon, K. E., Khalid, M. M., Tomar, S., Jimenez-Morales, D., Dunlap, M., Kaye, J. A., Shah, P. S., Finkbeiner, S., Krogan, N. J., and Ott, M. 2018. The cellular NMD pathway restricts Zika virus infection and is targeted by the viral capsid protein. mBio 9.

Fox, A. H., Nakagawa, S., Hirose, T., and Bond, C. S. 2018. Paraspeckles: where long noncoding RNA meets phase separation. Trends Biochem Sci 43:124-135.

Freire, M. A. 2014. Potyviral VPg and HC-Pro proteins and the cellular translation initiation factor elF(iso)4E interact with exoribonuclease Rrp6 and a small alpha-heat shock protein. Plant Mol Biol Rep 32:596-604.

Fros, J. J., Dietrich, I., Alshaikhahmed, K., Passchier, T. C., Evans, D. J., and Simmonds, P. 2017. CpG and UpA dinucleotides in both coding and non-coding regions of echovirus 7 inhibit replication initiation post-entry. Elife 6: e29112.

Fros, J. J., Domeradzka, N. E., Baggen, J., Geertsema, C., Flipse, J., Vlak, J. M., and Pijlman, G. P. 2012. Chikungunya virus nsP3 blocks stress granule assembly by recruitment of G3BP into cytoplasmic foci. J Virol 86:10873-10879.

Fujioka, Y., Utsumi, M., Ohba, Y., and Watanabe, Y. 2007. Location of a possible miRNA processing site in SmD3/SmB nuclear bodies in Arabidopsis. Plant and Cell Physiol 48:1243-1253.

Fukuhara, N., Ebert, J., Unterholzner, L., Lindner, D., Izaurralde, E., and Conti, E. 2005. SMG7 is a 14-33-like adaptor in the nonsense-mediated mRNA decay pathway. Mol Cell 17:537-547.

\section{G}

Galganski, L., Urbanek, M. O., and Krzyzosiak, W. J. 2017. Nuclear speckles: molecular organization, biological function and role in disease. Nucleic Acids Res 45:10350-10368.

Garcia, D., Garcia, S., and Voinnet, O. 2014. Nonsense-mediated decay serves as a general viral restriction mechanism in plants. Cell Host Microbe 16:391-402.

Garcia, D., Garcia, S., Pontier, D., Marchais, A., Renou, J. P., Lagrange, T., and Voinnet, O. 2012. Ago hook and RNA helicase motifs underpin dual roles for SDE3 in antiviral defense and silencing of nonconserved intergenic regions. Mol Cell 48:109-120. 
Garcia-Moreno, M., Noerenberg, M., Ni, S., Jarvelin, A. I., Gonzalez-Almela, E., Lenz, C. E., Bach-Pages, M., Cox, V., Avolio, R., Davis, T., Hester, S., Sohier, T. J. M., Li, B., Heikel, G., Michlewski, G., Sanz, M. A., Carrasco, L., Ricci, E. P., Pelechano, V., Davis, I., Fischer, B., Mohammed, S., and Castello, A. 2019. System-wide profiling of RNA-binding proteins uncovers key regulators of virus infection. Mol Cell 74:196-211.e111.

Garcin, D., Lezzi, M., Dobbs, M., Elliott, R. M., Schmaljohn, C., Kang, C. Y., and Kolakofsky, D. 1995. The 5 ' ends of Hantaan virus (Bunyaviridae) RNAs suggest a prime-and-realign mechanism for the initiation of RNA synthesis. J Virol 69:5754-5762.

Ge, Z. Y., Quek, B. L., Beemon, K. L., and Hogg, J. R. 2016. Polypyrimidine tract binding protein 1 protects mRNAs from recognition by the nonsense-mediated mRNA decay pathway. Elife 5 .

Geerts-Dimitriadou, C., Goldbach, R., and Kormelink, R. 2011a. Preferential use of RNA leader sequences during influenza A transcription initiation in vivo. Virology 409:27-32.

Geerts-Dimitriadou, C., Zwart, M. P., Goldbach, R., and Kormelink, R. 2011b. Base-pairing promotes leader selection to prime in vitro influenza genome transcription. Virology 409:17-26.

Geerts-Dimitriadou, C., Lu, Y. Y., Geertsema, C., Goldbach, R., and Kormelink, R. 2012. Analysis of the Tomato spotted wilt virus ambisense S RNA-encoded hairpin structure in translation. PLoS One 7:e31013.

Gerlach, P., Malet, H., Cusack, S., and Reguera, J. 2015. Structural insights into bunyavirus replication and its regulation by the vRNA promoter. Cell 161:1267-1279.

Ghoshal, B., and Sanfacon, H. 2014. Temperature-dependent symptom recovery in Nicotiana benthamiana plants infected with Tomato ringspot virus is associated with reduced translation of viral RNA2 and requires Argonaute 1. Virology 456:188-197.

Gilder, A. S., Do, P. M., Carrero, Z. I., Cosman, A. M., Broome, H. J., Velma, V., Martinez, L. A., and Hebert, M. D. 2011. Coilin participates in the suppression of RNA polymerase I in response to cisplatin-induced DNA damage. Mol Biol Cell 22:1070-1079.

Glick, E., Zrachya, A., Levy, Y., Mett, A., Gidoni, D., Belausov, E., Citovsky, V., and Gafni, Y. 2008. Interaction with host SGS3 is required for suppression of RNA silencing by Tomato yellow leaf curl virus V2 protein. Proc Natl Acad Sci USA 105:157-161.

Gogrefe, N., Reindl, S., Günther, S., and Rosenthal, M. 2019. Structure of a functional cap-binding domain in Rift Valley fever virus L protein. PLoS Pathog 15:e1007829.

Gonzalez, I., Martinez, L., Rakitina, D. V., Lewsey, M. G., Atencio, F. A., Llave, C., Kalinina, N. O., Carr, J. P., Palukaitis, P., and Canto, T. 2010. Cucumber mosaic virus $2 \mathrm{~b}$ protein subcellular targets and interactions: their significance to RNA silencing suppressor activity. Mol Plant Microbe Interact 23:294-303.

Gu, W., Gallagher, G. R., Dai, W., Liu, P., Li, R., Trombly, M. I., Gammon, D. B., Mello, C. C., Wang, J. P., and Finberg, R. W. 2015. Influenza A virus preferentially snatches noncoding RNA caps. RNA 21:2067-2075.

Gui, H., Lu, C. W., Adams, S., Stollar, V., and Li, M. L. 2010. hnRNP A1 interacts with the genomic and subgenomic RNA promoters of Sindbis virus and is required for the synthesis of $G$ and SG RNA. J Biomed Sci 17:59.

Guilligay, D., Tarendeau, F., Resa-Infante, P., Coloma, R., Crepin, T., Sehr, P., Lewis, J., Ruigrok, R. W., Ortin, J., Hart, D. J., and Cusack, S. 2008. The structural basis for cap binding by influenza virus polymerase subunit PB2. Nat Struct Mol Biol 15:500-506.

Guo, H., Song, X., Xie, C., Huo, Y., Zhang, F., Chen, X., Geng, Y., and Fang, R. 2013. Rice yellow stunt rhabdovirus protein 6 suppresses systemic RNA silencing by blocking RDR6-mediated secondary siRNA synthesis. Mol Plant Microbe Interact 26:927-936.

Guo, M., Liu, J. H., Ma, X., Luo, D. X., Gong, Z. H., and Lu, M. H. 2016. The plant heat stress transcription factors (HSFs): structure, regulation, and function in response to abiotic stresses. Front Plant Sci $7: 114$. 
Guo, T. W., Vimalesvaran, D., Thompson, J. R., Perry, K. L., and Krenz, B. 2015. Subcellular localization of grapevine red blotch-associated virus ORFs V2 and V3. Virus Genes 51:156-158.

Gutierrez-Beltran, E., Moschou, P. N., Smertenko, A. P., and Bozhkov, P. V. 2015. Tudor staphylococcal nuclease links formation of stress granules and processing bodies with mRNA catabolism in Arabidopsis. Plant Cell 27:926-943.

\section{H}

Hafren, A., Eskelin, K., and Makinen, K. 2013. Ribosomal protein P0 promotes Potato virus A infection and functions in viral translation together with VPg and elF(iso)4E. J Virol 87:4302-4312.

Hafren, A., Lohmus, A., and Makinen, K. 2015. Formation of Potato Virus A-Induced RNA granules and viral translation are interrelated processes required for optimal virus accumulation. PLoS Pathog 11:e1005314.

Hamada, T., Yako, M., Minegishi, M., Sato, M., Kamei, Y., Yanagawa, Y., Toyooka, K., Watanabe, Y., and Hara-Nishimura, I. 2018. Stress granule formation is induced by a threshold temperature rather than a temperature difference in Arabidopsis. J Cell Sci 131.

Hannon, G. J. 2002. RNA interference. Nature 418:244-251.

Haque, A., and Mir, M. A. 2010. Interaction of hantavirus nucleocapsid protein with ribosomal protein S19. J Virol 84:12450-12453.

Hastie, K. M., Liu, T., Li, S., King, L. B., Ngo, N., Zandonatti, M. A., Woods, V. L., Jr., de la Torre, J. C., and Saphire, E. O. 2011. Crystal structure of the Lassa virus nucleoprotein-RNA complex reveals a gating mechanism for RNA binding. Proc Natl Acad Sci USA 108:19365-19370.

Heath, C. M., Windsor, M., and Wileman, T. 2001. Aggresomes resemble sites specialized for virus assembly. J Cell Biol 153:449-455.

Hebner, C. M., and Laimins, L. A. 2006. Human papillomaviruses: basic mechanisms of pathogenesis and oncogenicity. Rev Med Virol 16:83-97.

Herranz, M. C., Pallas, V., and Aparicio, F. 2012. Multifunctional roles for the N-terminal basic motif of Alfalfa mosaic virus coat protein: nucleolar/cytoplasmic shuttling, modulation of RNA-binding activity, and virion formation. Mol Plant Microbe Interact 25:1093-1103.

Hofer, C. T., Jolmes, F., Haralampiev, I., Veit, M., and Herrmann, A. 2017. Influenza A virus nucleoprotein targets subnuclear structures. Cell Microbiol 19 (4), e12679.

Hopkins, K. C., McLane, L. M., Maqbool, T., Panda, D., Gordesky-Gold, B., and Cherry, S. 2013. A genome-wide RNAi screen reveals that mRNA decapping restricts bunyaviral replication by limiting the pools of Dcp2-accessible targets for cap-snatching. Genes Dev 27:1511-1525.

Hyodo, K., and Okuno, T. 2020. Hijacking of host cellular components as proviral factors by plantinfecting viruses. Adv Virus Res 107:37-86.

\section{I}

Ingelfinger, D., Arndt-Jovin, D. J., Luhrmann, R., and Achsel, T. 2002. The human LSm1-7 proteins colocalize with the mRNA-degrading enzymes Dcp1/2 and Xrnl in distinct cytoplasmic foci. Rna 8:1489-1501.

\section{J}

Jaag, H. M., and Nagy, P. D. 2009. Silencing of Nicotiana benthamiana Xrn4p exoribonuclease promotes tombusvirus RNA accumulation and recombination. Virology 386:344-352.

Jakymiw, A., Lian, S., Eystathioy, T., Li, S., Satoh, M., Hamel, J. C., Fritzler, M. J., and Chan, E. K. L. 2005. Disruption of GW bodies impairs mammalian RNA interference. Nature Cell Biology 7:1267-1274.

Jeeva, S., Cheng, E., Ganaie, S. S., and Mir, M. A. 2017a. Crimean-Congo hemorrhagic fever virus nucleocapsid protein augments mrna translation. J Virol 91. 
Jeeva, S., Pador, S., Voss, B., Ganaie, S. S., and Mir, M. A. 2017b. Crimean-Congo hemorrhagic fever virus nucleocapsid protein has dual RNA binding modes. PLoS One 12:e0184935.

Jeeva, S., Mir, S., Velasquez, A., Weathers, B. A., Leka, A., Wu, S., Sevarany, A. T., and Mir, M. 2019. Hantavirus rdrp requires a host cell factor for cap snatching. J Virol 93.

\section{K}

Kainz, M., and Hoopes, B. C. 2009. Identification of candidate Arabidopsis thaliana proteins involved in the replication cycle of Tomato spotted wilt virus. Abstracts of the General Meeting of the American Society for Microbiology 109.

Kalinina, N. O., Makarova, S., Makhotenko, A., Love, A. J., and Taliansky, M. 2018. The multiple functions of the nucleolus in plant development, disease and stress responses. Front Plant Sci 9.

Kedersha, N., Stoecklin, G., Ayodele, M., Yacono, P., Lykke-Andersen, J., Fritzler, M. J., Scheuner, D., Kaufman, R. J., Golan, D. E., and Anderson, P. 2005. Stress granules and processing bodies are dynamically linked sites of mRNP remodeling. J Cell Biol 169:871-884.

Kedersha, N. L., Gupta, M., Li, W., Miller, I., and Anderson, P. 1999. RNA-binding proteins TIA-1 and TIAR link the phosphorylation of elF-2 alpha to the assembly of mammalian stress granules. J Cell Biol 147:1431-1442.

Khaperskyy, D. A., Hatchette, T. F., and McCormick, C. 2012. Influenza A virus inhibits cytoplasmic stress granule formation. Faseb J. 26:1629-1639.

Khong, A., Matheny, T., Jain, S., Mitchell, S. F., Wheeler, J. R., and Parker, R. 2017. The stress granule transcriptome reveals principles of mRNA accumulation in stress granules. Mol Cell 68:808-820 e805.

Kikkert, M., Verschoor, A., Kormelink, R., Rottier, P., and Goldbach, R. 2001. Tomato spotted wilt virus glycoproteins exhibit trafficking and localization signals that are functional in mammalian cells. J Virol 75:1004-1012.

Kim, S. H., Ryabov, E. V., Brown, J. W., and Taliansky, M. 2004. Involvement of the nucleolus in plant virus systemic infection. Biochem Soc Trans 32:557-560.

Kim, S. H., Macfarlane, S., Kalinina, N. O., Rakitina, D. V., Ryabov, E. V., Gillespie, T., Haupt, S., Brown, J. W., and Taliansky, M. 2007a. Interaction of a plant virus-encoded protein with the major nucleolar protein fibrillarin is required for systemic virus infection. Proc Natl Acad Sci USA 104:11115-11120.

Kim, S. H., Ryabov, E. V., Kalinina, N. O., Rakitina, D. V., Gillespie, T., MacFarlane, S., Haupt, S., Brown, J. W., and Taliansky, M. 2007b. Cajal bodies and the nucleolus are required for a plant virus systemic infection. EMBO J 26:2169-2179.

Kim, Y. K., and Maquat, L. E. 2019. UPFront and centre in RNA decay: UPF1 in nonsense-mediated mRNA decay and beyond. Rna 25:407-422.

Koppstein, D., Ashour, J., and Bartel, D. P. 2015. Sequencing the cap-snatching repertoire of H1N1 influenza provides insight into the mechanism of viral transcription initiation. Nucleic Acids Res 43:5052-5064.

Kormelink, R., Garcia, M. L., Goodin, M., Sasaya, T., and Haenni, A. L. 2011. Negative-strand RNA viruses: the plant-infecting counterparts. Virus Res 162:184-202.

Krapp, S., Greiner, E., Amin, B., Sonnewald, U., and Krenz, B. 2017. The stress granule component G3BP is a novel interaction partner for the nuclear shuttle proteins of the nanovirus pea necrotic yellow dwarf virus and geminivirus abutilon mosaic virus. Virus Res 227:6-14.

Kulkarni, M., Ozgur, S., and Stoecklin, G. 2010. On track with P bodies. Biochem Soc Trans 38:242-251.

Kumakura, N., Takeda, A., Fujioka, Y., Motose, H., Takano, R., and Watanabe, Y. 2009. SGS3 and RDR6 interact and colocalize in cytoplasmic SGS3/RDR6-bodies. FEBS Letters 583:1261-1266.

Kurihara, Y., Takashi, Y., and Watanabe, Y. 2006. The interaction between DCL1 and HYL1 is important for efficient and precise processing of pri-miRNA in plant microRNA biogenesis. Rna 12:206-212. 
Lahmy, S., Pontier, D., Cavel, E., Vega, D., El-Shami, M., Kanno, T., and Lagrange, T. 2009. PolV(PolIVb) function in RNA-directed DNA methylation requires the conserved active site and an additional plant-specific subunit. Proc Natl Acad Sci USA 106:941-946.

Law, L. M. J., Razooky, B. S., Li, M. M. H., You, S., Jurado, A., Rice, C. M., and MacDonald, M. R. 2019. ZAP's stress granule localization is correlated with its antiviral activity and induced by virus replication. PLoS Pathog 15:e1007798.

Lee, J. H., Kim, S. H., Pascua, P. N., Song, M. S., Baek, Y. H., Jin, X., Choi, J. K., Kim, C. J., Kim, H., and Choi, Y. K. 2010. Direct interaction of cellular hnRNP-F and NS1 of influenza A virus accelerates viral replication by modulation of viral transcriptional activity and host gene expression. Virology 397:89-99.

Li, C. F., Pontes, O., El-Shami, M., Henderson, I. R., Bernatavichute, Y. V., Chan, S. W. L., Lagrange, T., Pikaard, C. S., and Jacobsen, S. E. 2006. An Argonaute4-containing nuclear processing centre colocalized with Cajal bodies in Arabidopsis thaliana. Cell 126:93-106.

Li, F., Huang, C., Li, Z., and Zhou, X. 2014a. Suppression of RNA silencing by a plant DNA virus satellite requires a host calmodulin-like protein to repress RDR6 expression. PLoS Pathog 10:e1003921.

Li, H. 2018. Minimap2: pairwise alignment for nucleotide sequences. Bioinformatics 34:3094-3100.

$\mathrm{Li}, \mathrm{H}$. , and Durbin, R. 2009. Fast and accurate short read alignment with Burrows-Wheeler transform. Bioinformatics 25:1754-1760.

Li, J., Hu, S., Xu, F., Mei, S., Liu, X., Yin, L., Zhao, F., Zhao, X., Sun, H., Xiong, Z., Zhang, D., Cen, S., Wang, J., Liang, C., and Guo, F. 2019a. MOV10 sequesters the RNP of influenza A virus in the cytoplasm and is antagonized by viral NS1 protein. Biochem J 476:467-481.

Li, L., Dai, H., Nguyen, A. P., Hai, R., and Gu, W. 2020. Influenza A virus utilizes noncanonical capsnatching to diversify its mRNA/ncRNA. RNA 26:1170-1183.

Li, M., Johnson, J. R., Truong, B., Kim, G., Weinbren, N., Dittmar, M., Shah, P. S., Von Dollen, J., Newton, B. W., Jang, G. M., Krogan, N. J., Cherry, S., and Ramage, H. 2019b. Identification of antiviral roles for the exon-junction complex and nonsense-mediated decay in flaviviral infection. Nature microbiology 4:985-995.

Li, M. L., Rao, P., and Krug, R. M. 2001. The active sites of the influenza cap-dependent endonuclease are on different polymerase subunits. EMBO J 20:2078-2086.

Li, R., Weldegergis, B. T., Li, J., Jung, C., Qu, J., Sun, Y., Qian, H., Tee, C., van Loon, J. J., Dicke, M., Chua, N. H., Liu, S. S., and Ye, J. 2014b. Virulence factors of geminivirus interact with MYC2 to subvert plant resistance and promote vector performance. Plant Cell 26:4991-5008.

Li, W., Li, Y., Kedersha, N., Anderson, P., Emara, M., Swiderek, K. M., Moreno, G. T., and Brinton, M. A. 2002a. Cell proteins TIA-1 and TIAR interact with the 3' stem-loop of the West Nile virus complementary minus-strand RNA and facilitate virus replication. J Virol 76:11989-12000.

Li, X. D., Mäkelä, T. P., Guo, D., Soliymani, R., Koistinen, V., Vapalahti, O., Vaheri, A., and Lankinen, H. 2002b. Hantavirus nucleocapsid protein interacts with the Fas-mediated apoptosis enhancer Daxx. J Gen Virol 83:759-766.

Li, Z., Zeng, W., Ye, S., Lv, J., Nie, A., Zhang, B., Sun, Y., Han, H., and He, Q. 2018a. Cellular hnRNP A1 interacts with nucleocapsid protein of Porcine epidemic diarrhea virus and impairs viral replication. Viruses 10 .

Li, Z., Zhang, Y., Jiang, Z., Jin, X., Zhang, K., Wang, X., Han, C., Yu, J., and Li, D. 2018b. Hijacking of the nucleolar protein fibrillarin by TGB1 is required for cell-to-cell movement of Barley stripe mosaic virus. Mol Plant Pathol 19:1222-1237.

Linero, F., Welnowska, E., Carrasco, L., and Scolaro, L. 2013. Participation of elF4F complex in Junin virus infection: blockage of elF4E does not impair virus replication. Cell Microbiol 15:1766-1782. 
Linero, F. N., Thomas, M. G., Boccaccio, G. L., and Scolaro, L. A. 2011. Junin virus infection impairs stressgranule formation in Vero cells treated with arsenite via inhibition of elF2alpha phosphorylation. J Gen Virol 92:2889-2899.

Lingel, A., and Sattler, M. 2005. Novel modes of protein-RNA recognition in the RNAi pathway. Curr Opin Struct Biol 15:107-115.

Liu, J., Valencia-Sanchez, M. A., Hannon, G. J., and Parker, R. 2005. MicroRNA-dependent localization of targeted mRNAs to mammalian P bodies. Nat Cell Biol 7:719-723.

Liu, J. L., and Gall, J. G. 2007. U bodies are cytoplasmic structures that contain uridine-rich small nuclear ribonucleoproteins and associate with P bodies. Proc Natl Acad Sci USA 104:11655-11659.

Liu, X., Xiong, G., Qiu, P., Du, Z., Kormelink, R., Zheng, L., Zhang, J., Ding, X., Yang, L., Zhang, S., and $\mathrm{Wu}$, Z. 2016. Inherent properties not conserved in other tenuiviruses increase priming and realignment cycles during transcription of Rice stripe virus. Virology 496:287-298.

Lloyd, R. E. 2015. Nuclear proteins hijacked by mammalian cytoplasmic plus strand RNA viruses. Virology 479-480:457-474.

Lloyd, R. E. 2016. Enterovirus control of translation and rna granule stress responses. Viruses 8:93.

Lopez-Lastra, M., Ramdohr, P., Letelier, A., Vallejos, M., Vera-Otarola, J., and Valiente-Echeverria, F. 2010. Translation initiation of viral mRNAs. Rev Med Virol 20:177-195.

Lopez-Turiso, J. A., Martinez, C., Tanaka, T., and Ortin, J. 1990. The synthesis of influenza virus negativestrand RNA takes place in insoluble complexes present in the nuclear matrix fraction. Virus Res 16:325-337.

Loschi, M., Leishman, C. C., Berardone, N., and Boccaccio, G. L. 2009. Dynein and kinesin regulate stress-granule and P body dynamics. J Cell Sci 122:3973-3982.

Love, A. J., Yu, C., Petukhova, N. V., Kalinina, N. O., Chen, J., and Taliansky, M. E. 2017. Cajal bodies and their role in plant stress and disease responses. RNA Biol 14:779-790.

Love, M. I., Huber, W., and Anders, S. 2014. Moderated estimation of fold change and dispersion for RNA-seq data with DESeq2. Genome Biol 15:550.

Luan, J. B., Li, J. M., Varela, N., Wang, Y. L., Li, F. F., Bao, Y. Y., Zhang, C. X., Liu, S. S., and Wang, X. W. 2011. Global analysis of the transcriptional response of whitefly to Tomato yellow leaf curl China virus reveals the relationship of coevolved adaptations. J Virol 85:3330-3340.

Lukhovitskaya, N. I., Cowan, G. H., Vetukuri, R. R., Tilsner, J., Torrance, L., and Savenkov, E. I. 2015. Importin-alpha-mediated nucleolar localization of potato mop-top virus TRIPLE GENE BLOCK1 (TGB1) protein facilitates virus systemic movement, whereas TGB1 self-interaction is required for cell-to-cell movement in Nicotiana benthamiana. Plant Physiol 167:738-752.

Lykke-Andersen, J. 2002. Identification of a Human Decapping Complex Associated with hUpf Proteins in Nonsense-Mediated Decay. Mol Cell Biol 22:8114-8121.

Lykke-Andersen, J., and Wagner, E. 2005. Recruitment and activation of mRNA decay enzymes by two ARE-mediated decay activation domains in the proteins TTP and BRF-1. Genes Dev 19:351-361.

\section{M}

Ma, X., Zhou, Y., and Moffett, P. 2019. Alterations in cellular RNA decapping dynamics affect Tomato spotted wilt virus cap snatching and infection in Arabidopsis. New Phytol 224:789-803.

Machyna, M., Neugebauer, K. M., and Staněk, D. 2015. Coilin: The first 25 years. RNA Biol 12:590-596.

Maeda, A., Lee, B. H., Yoshimatsu, K., Saijo, M., Kurane, I., Arikawa, J., and Morikawa, S. 2003. The intracellular association of the nucleocapsid protein (NP) of hantaan virus (HTNV) with small ubiquitin-like modifier-1 (SUMO-1) conjugating enzyme 9 (Ubc9). Virology 305:288-297.

Mäkinen, K., Lõhmus, A., and Pollari, M. 2017. Plant RNA regulatory network and RNA granules in virus infection. Front Plant Sci 8.

Malinowska, M., Niedźwiedzka-Rystwej, P., Tokarz-Deptuła, B., and Deptuła, W. 2016. Stress granules (SG) and processing bodies (PB) in viral infections. Acta Biochim Pol 63:183-188. 
Mao, Y. S., Zhang, B., and Spector, D. L. 2011. Biogenesis and function of nuclear bodies. Trends Genet 27:295-306.

Martin, M. 2011. Cutadapt removes adapter sequences from high-throughput sequencing reads. EMBnet.journal; Vol 17, No 1: Next Generation Sequencing Data Analysis.

Martinez de Alba, A. E., Moreno, A. B., Gabriel, M., Mallory, A. C., Christ, A., Bounon, R., Balzergue, S., Aubourg, S., Gautheret, D., Crespi, M. D., Vaucheret, H., and Maizel, A. 2015. In plants, decapping prevents RDR6-dependent production of small interfering RNAs from endogenous mRNAs. Nucleic Acids Res 43:2902-2913.

Matthews, J. D., and Frey, T. K. 2012. Analysis of subcellular G3BP redistribution during rubella virus infection. J Gen Virol 93:267-274.

May, J. P., Yuan, X., Sawicki, E., and Simon, A. E. 2018. RNA virus evasion of nonsense-mediated decay. PLoS Pathog 14:e1007459.

McInerney, G. M., Kedersha, N. L., Kaufman, R. J., Anderson, P., and Liljestrom, P. 2005. Importance of elF2 alpha phosphorylation assembly in alphavirus translation and stress granule regulation. Mol Biol Cell 16:3753-3763.

Meteignier, L. V., Zhou, J., Cohen, M., Bhattacharjee, S., Brosseau, C., Chan, M. G., Robatzek, S., and Moffett, P. 2016. NB-LRR signalling induces translational repression of viral transcripts and the formation of RNA processing bodies through mechanisms differing from those activated by UV stress and RNAi. J Exp Bot 67:2353-2366.

Meyer, B. J., and Southern, P. J. 1993. Concurrent sequence analysis of 5' and 3' RNA termini by intramolecular circularization reveals $5^{\prime}$ nontemplated bases and $3^{\prime}$ terminal heterogeneity for lymphocytic choriomeningitis virus mrnas. j virol 67:2621-2627.

Mir, M. A., Sheema, S., Haseeb, A., and Haque, A. 2010. hantavirus nucleocapsid Protein Has Distinct m7G Cap- and RNA-binding sites. J Biol Chem 285:11357-11368.

Mir, M. A., Duran, W. A., Hjelle, B. L., Ye, C., and Panganiban, A. T. 2008b. Storage of cellular 5' mRNA caps in P bodies for viral cap-snatching. Proc Natl Acad Sci USA 105:19294-19299.

Miyamoto, Y., Yamada, K., and Yoneda, Y. 2016. Importin alpha: a key molecule in nuclear transport and non-transport functions. J Biochem 160:69-75.

Mo, Q., Xu, Z., Deng, F., Wang, H., and Ning, Y. J. 2020. Host restriction of emerging high-pathogenic bunyaviruses via MOV10 by targeting viral nucleoprotein and blocking ribonucleoprotein assembly. PLoS Pathog 16:e1009129.

Mochizuki, T., Ogata, Y., Hirata, Y., and Ohki, S. T. 2014. Quantitative transcriptional changes associated with chlorosis severity in mosaic leaves of tobacco plants infected with Cucumber mosaic virus. Mol Plant Pathol 15:242-254.

Mok, B. W., Song, W., Wang, P., Tai, H., Chen, Y., Zheng, M., Wen, X., Lau, S. Y., Wu, W. L., Matsumoto, K., Yuen, K. Y., and Chen, H. 2012. The NS1 protein of influenza A virus interacts with cellular processing bodies and stress granules through RNA-associated protein 55 (RAP55) during virus infection. J Virol 86:12695-12707.

Moore, M. J. 2005. From birth to death: the complex lives of eukaryotic mRNAs. Science 309:1514-1518.

Moore, S., Rabichow, B. E., and Sattler, R. 2020. The Hitchhiker's Guide to Nucleocytoplasmic Trafficking in Neurodegeneration. Neurochem Res 45:1306-1327.

Morimoto, M., and Boerkoel, C. F. 2013. The role of nuclear bodies in gene expression and disease. Biology 2:976-1033.

Moser, J. J., Eystathioy, T., Chan, E. K. L., and Fritzler, M. J. 2007. Markers of mRNA stabilization and degradation, and RNAi within astrocytoma GW bodies. Neurochem Res 85:3619-3631.

$\mathbf{N}$

Ng, L. F., Chan, M., Chan, S. H., Cheng, P. C., Leung, E. H., Chen, W. N., and Ren, E. C. 2005. Host heterogeneous ribonucleoprotein K (hnRNPK) as a potential target to suppress hepatitis B virus replication. PLoS Med 2:e163. 
Niu, F., Shaw, N., Wang, Y. E., Jiao, L., Ding, W., Li, X., Zhu, P., Upur, H., Ouyang, S., Cheng, G., and Liu, Z. J. 2013. Structure of the Leanyer orthobunyavirus nucleoprotein-RNA complex reveals unique architecture for RNA encapsidation. Proc Natl Acad Sci USA 110:9054-9059.

Nizami, Z., Deryusheva, S., and Gall, J. G. 2010a. The Cajal body and histone locus body. Cold Spring Harb Perspect Biol 2:a000653.

Nizami, Z. F., Deryusheva, S., and Gall, J. G. 2010b. Cajal bodies and histone locus bodies in Drosophila and Xenopus. Cold Spring Harb Symp Quant Biol 75:313-320.

Novoa, R. R., Calderita, G., Arranz, R., Fontana, J., Granzow, H., and Risco, C. 2005. Virus factories: associations of cell organelles for viral replication and morphogenesis. Biol Cell 97:147-172.

0

Okano, Y., Senshu, H., Hashimoto, M., Neriya, Y., Netsu, O., Minato, N., Yoshida, T., Maejima, K., Oshima, K., Komatsu, K., Yamaji, Y., and Namba, S. 2014. In planta recognition of a double-stranded RNA synthesis protein complex by a potexviral RNA silencing suppressor. Plant Cell 26:2168-2183.

Okonski, K. M., and Samuel, C. E. 2013. Stress granule formation induced by Measles virus is protein kinase pkr dependent and impaired by RNA adenosine deaminase ADAR1. J Virol 87:756-766.

Olschewski, S., Cusack, S., and Rosenthal, M. 2020. The Cap-Snatching Mechanism of Bunyaviruses. Trends Microbiol 28:293-303.

Onomoto, K., Yoneyama, M., Fung, G., Kato, H., and Fujita, T. 2014. Antiviral innate immunity and stress granule responses. Trends Immunol 35:420-428.

Onomoto, K., Jogi, M., Yoo, J. S., Narita, R., Morimoto, S., Takemura, A., Sambhara, S., Kawaguchi, A., Osari, S., Nagata, K., Matsumiya, T., Namiki, H., Yoneyama, M., and Fujita, T. 2012. Critical role of an antiviral stress granule containing RIG-I and PKR in viral detection and innate immunity. Plos One 7 (8), e43031.

Orban, T. I., and Izaurralde, E. 2005. Decay of mRNAs targeted by RISC requires XRN1, the Ski complex, and the exosome. Rna 11:459-469.

Ortin, J., and Martin-Benito, J. 2015. The RNA synthesis machinery of negative-stranded RNA viruses. Virology 479-480:532-544.

Osborne, M. J., and Borden, K. L. 2015. The eukaryotic translation initiation factor elF4E in the nucleus: taking the road less traveled. Immunol Rev 263:210-223.

\section{$\mathbf{P}$}

Paape, M., Solovyev, A. G., Erokhina, T. N., Minina, E. A., Schepetilnikov, M. V., Lesemann, D. E., Schiemann, J., Morozov, S. Y., and Kellmann, J. W. 2006. At-4/1, an interactor of the Tomato spotted wilt virus movement protein, belongs to a new family of plant proteins capable of directed intra- and intercellular trafficking. Mol Plant Microbe Interact 19:874-883.

Pager, C. T., Schutz, S., Abraham, T. M., Luo, G., and Sarnow, P. 2013. Modulation of hepatitis C virus RNA abundance and virus release by dispersion of processing bodies and enrichment of stress granules. Virology 435:472-484.

Panas, M. D., Varjak, M., Lulla, A., Eng, K. E., Merits, A., Karlsson Hedestam, G. B., and Mclnerney, G. M. 2012. Sequestration of G3BP coupled with efficient translation inhibits stress granules in Semliki Forest virus infection. Mol Biol Cell 23:4701-4712.

Panganiban, A. T., and Mir, M. A. 2009. Bunyavirus N: elF4F surrogate and cap-guardian. Cell Cycle 8:1332-1337.

Parker, R., and Sheth, U. 2007. P bodies and the control of mRNA translation and degradation. Mol Cell 25:635-646.

Patel, P. H., Barbee, S. A., and Blankenship, J. T. 2016. GW-bodies and P bodies constitute two separate pools of sequestered non-translating RNAs. PLoS One 11:e0150291. 
Pauley, K. M., Eystathioy, T., Jakymiw, A., Hamel, J. C., Fritzler, M. J., and Chan, E. K. L. 2006. Formation of $\mathrm{GW}$ bodies is a consequence of microRNA genesis. EMBO reports 7:904-910.

Pendle, A. F., Clark, G. P., Boon, R., Lewandowska, D., Lam, Y. W., Andersen, J., Mann, M., Lamond, A. I., Brown, J. W., and Shaw, P. J. 2005. Proteomic analysis of the Arabidopsis nucleolus suggests novel nucleolar functions. Mol Biol Cell 16:260-269.

Peng, J., Yang, J., Yan, F., Lu, Y., Jiang, S., Lin, L., Zheng, H., Chen, H., and Chen, J. 2011. Silencing of NbXrn4 facilitates the systemic infection of Tobacco mosaic virus in Nicotiana benthamiana. Virus Res 158:268-270.

Perez-Canamas, M., and Hernandez, C. 2018. new insights into the nucleolar localization of a plant RNA virus-encoded protein that acts in both RNA packaging and RNA silencing suppression: involvement of importins alpha and relevance for viral infection. Mol Plant Microbe Interact 31:1134-1144.

Pfaff, C., Ehrnsberger, H. F., Flores-Tornero, M., Sorensen, B. B., Schubert, T., Langst, G., Griesenbeck, J., Sprunck, S., Grasser, M., and Grasser, K. D. 2018. ALY RNA-binding proteins are required for nucleocytosolic mRNA transport and modulate plant growth and development. Plant Physiol 177:226-240.

Pflug, A., Guilligay, D., Reich, S., and Cusack, S. 2014. Structure of influenza A polymerase bound to the viral RNA promoter. Nature 516:355-360.

Pico, S., Ortiz-Marchena, M. I., Merini, W., and Calonje, M. 2015. Deciphering the role of polycomb repressive complex1 variants in regulating the acquisition of flowering competence in Arabidopsis. Plant Physiol 168:1286-1297.

Pillai, R. S., Bhattacharyya, S. N., Artus, C. G., Zoller, T., Cougot, N., Basyuk, E., Bertrand, E., and Filipowicz, W. 2005. Inhibition of translational initiation by Let-7 MicroRNA in human cells. Science 309:1573-1576.

Poblete-Durán, N., Prades-Pérez, Y., Vera-Otarola, J., Soto-Rifo, R., and Valiente-Echeverría, F. 2016. Who regulates whom? an overview of RNA granules and viral infections. Viruses 8:180.

Pomeranz, M., Lin, P. C., Finer, J., and Jang, J. C. 2010. AtTZF gene family localizes to cytoplasmic foci. Plant Signal Behav 5:190-192.

Pontes, O., and Pikaard, C. S. 2008. siRNA and miRNA processing: new functions for Cajal bodies. Curr Opin Genet Dev 18:197-203.

Pontes, O., Li, C. F., Costa Nunes, P., Haag, J., Ream, T., Vitins, A., Jacobsen, S. E., and Pikaard, C. S. 2006. The Arabidopsis chromatin-modifying nuclear siRNA pathway involves a nucleolar RNA processing centre. Cell 126:79-92.

Prasch, C. M., and Sonnewald, U. 2013. Simultaneous application of heat, drought, and virus to Arabidopsis plants reveals significant shifts in signalling networks. Plant Physiol 162:1849-1866.

Protter, D. S. W., and Parker, R. 2016. principles and properties of stress granules. Trends Cell Biol 26:668-679.

\section{Q}

Quinlan, A. R. 2014. BEDTools: The swiss-army tool for genome feature analysis. Curr Protoc Bioinformatics 47:11.12.11-11.12.34.

\section{$\mathbf{R}$}

Rajamaki, M. L., Streng, J., and Valkonen, J. P. 2014. Silencing suppressor protein VPg of a potyvirus interacts with the plant silencing-related protein SGS3. Mol Plant Microbe Interact 27:1199-1210.

Raju, R., Raju, L., Hacker, D., Garcin, D., Compans, R., and Kolakofsky, D. 1990. Nontemplated bases at the $5^{\prime}$ ends of Tacaribe virus mRNAs. Virology 174:53-59.

Ramanathan, A., Robb, G. B., and Chan, S. H. 2016. mRNA capping: biological functions and applications. Nucleic Acids Res 44:7511-7526. 
Ramanathan, H. N., Chung, D. H., Plane, S. J., Sztul, E., Chu, Y. K., Guttieri, M. C., McDowell, M., Ali, G., and Jonsson, C. B. 2007. Dynein-dependent transport of the hantaan virus nucleocapsid protein to the endoplasmic reticulum-Golgi intermediate compartment. J Virol 81:8634-8647.

Raoult, D., and Forterre, P. 2008. Redefining viruses: lessons from Mimivirus. Nat Rev Microbiol 6:315-319.

Ravkov, E. V., and Compans, R. W. 2001. Hantavirus nucleocapsid protein is expressed as a membraneassociated protein in the perinuclear region. J Virol 75:1808-1815.

Reguera, J., Weber, F., and Cusack, S. 2010. Bunyaviridae RNA polymerases (L-protein) have an $\mathrm{N}$-terminal, influenza-like endonuclease domain, essential for viral cap-dependent transcription. PLoS Pathog 6:e1001101.

Reguera, J., Gerlach, P., and Cusack, S. 2016. Towards a structural understanding of RNA synthesis by negative strand RNA viral polymerases. Curr Opin Struct Biol 36:75-84.

Reich, S., Guilligay, D., Pflug, A., Malet, H., Berger, I., Crepin, T., Hart, D., Lunardi, T., Nanao, M., Ruigrok, R. W., and Cusack, S. 2014. Structural insight into cap-snatching and RNA synthesis by influenza polymerase. Nature 516:361-366.

Reichelt, M., Stertz, S., Krijnse-Locker, J., Haller, O., and Kochs, G. 2004. Missorting of LaCrosse virus nucleocapsid protein by the interferon-induced MxA GTPase involves smooth ER membranes. Traffic 5:772-784.

Reineke, L. C., and Lloyd, R. E. 2013a. Diversion of stress granules and P bodies during viral infection. Virology 436:255-267.

Reineke, L. C., and Lloyd, R. E. 2013b. Diversion of stress granules and P bodies during viral infection. Virology 436:255-267.

Reyes, J. C. 2001. PML and COP1--two proteins with much in common. Trends Biochem Sci 26:18-20.

Ribeiro, D., Foresti, O., Denecke, J., Wellink, J., Goldbach, R., and Kormelink, R. J. M. 2008. Tomato spotted wilt virus glycoproteins induce the formation of endoplasmic reticulum- and Golgiderived pleomorphic membrane structures in plant cells. J Gen Virol 89:1811-1818.

Rodrigo-Peiris, T., Xu, X. M., Zhao, Q., Wang, H.-J., and Meier, I. 2011. RanGAP is required for postmeiotic mitosis in female gametophyte development in Arabidopsis thaliana. J Exp Bot 62:27052714.

Rognes, T., Flouri, T., Nichols, B., Quince, C., and Mahe, F. 2016. VSEARCH: a versatile open source tool for metagenomics. PeerJ 4:e2584.

Rosenthal, M., Gogrefe, N., Vogel, D., Reguera, J., Rauschenberger, B., Cusack, S., Gunther, S., and Reindl, S. 2017. Structural insights into reptarenavirus cap-snatching machinery. PLoS Pathog 13:e1006400.

\section{S}

Sainsbury, F., Thuenemann, E. C., and Lomonossoff, G. P. 2009. pEAQ: versatile expression vectors for easy and quick transient expression of heterologous proteins in plants. Plant Biotechnol J 7:682693.

Sang Hyon Kim, E. V. R., Natalia O Kalinina, Daria V Rakitina, Trudi Gillespie, Stuart MacFarlane, Sophie Haupt, John WS Brown, and and Michael Taliansky. 2009. Cajal bodies and the nucleolus are required for a plant virus systemic infection. EMBO J 26, 2169-2179.

Schöffl, F., Prändl, R., and Reindl, A. 1998. Regulation of the heat-shock response. Plant Physiol 117:1135-1141.

Scholte, F. E. M., Tas, A., Albulescu, I. C., Zusinaite, E., Merits, A., Snijder, E. J., and van Hemert, M. J. 2015. Stress Granule Components G3BP1 and G3BP2 Play a Proviral Role Early in Chikungunya Virus Replication. J Virol 89:4457-4469.

Scholthof, K. B., Adkins, S., Czosnek, H., Palukaitis, P., Jacquot, E., Hohn, T., Hohn, B., Saunders, K., Candresse, T., Ahlquist, P., Hemenway, C., and Foster, G. D. 2011. Top 10 plant viruses in molecular plant pathology. Mol Plant Pathol 12:938-954. 
Semashko, M. A., Gonzalez, I., Shaw, J., Leonova, O. G., Popenko, V. I., Taliansky, M. E., Canto, T., and Kalinina, N. O. 2012. The extreme N-terminal domain of a hordeivirus TGB1 movement protein mediates its localization to the nucleolus and interaction with fibrillarin. Biochimie 94:1180-1188.

Serman, A., Le Roy, F., Aigueperse, C., Kress, M., Dautry, F., and Weil, D. 2007. GW body disassembly triggered by siRNAs independently of their silencing activity. Nucleic Acids Res 35:4715-4727.

Shatkin, A. J. 1976. Capping of eucaryotic mRNAs. Cell 9:645-653.

Shaw, J., Love, A. J., Makarova, S. S., Kalinina, N. O., Harrison, B. D., and Taliansky, M. E. 2014. Coilin, the signature protein of Cajal bodies, differentially modulates the interactions of plants with viruses in widely different taxa. Nucleus 5:85-94.

Sheth, U., and Parker, R. 2003. Decapping and decay of messenger RNA occur in cytoplasmic processing bodies. Science 300:805-808.

Shi, S. T., Yu, G. Y., and Lai, M. M. 2003. Multiple type A/B heterogeneous nuclear ribonucleoproteins (hnRNPs) can replace hnRNP A1 in mouse hepatitis virus RNA synthesis. J Virol 77:10584-10593.

Shyu, A. B., Wilkinson, M. F., and van Hoof, A. 2008. Messenger RNA regulation: to translate or to degrade. EMBO J 27:471-481.

Sikora, D., Rocheleau, L., Brown, E. G., and Pelchat, M. 2017. Influenza A virus cap-snatches host RNAs based on their abundance early after infection. Virology 509:167-177.

Snippe, M., Borst, J. W., Goldbach, R., and Kormelink, R. 2005. The use of fluorescence microscopy to visualise homotypic interactions of Tomato spotted wilt virus nucleocapsid protein in living cells. J Virol Methods 125:15-22.

Snippe, M., Willem Borst, J., Goldbach, R., and Kormelink, R. 2007. Tomato spotted wilt virus Gc and N proteins interact in vivo. Virology 357:115-123.

Song, L., Han, M. H., Lesicka, J., and Fedoroff, N. 2007. Arabidopsis primary microRNA processing proteins HYL1 and DCL1 define a nuclear body distinct from the Cajal body. Proc Natl Acad Sci USA 104:5437-5442.

Sorenson, R., and Bailey-Serres, J. 2014. Selective mRNA sequestration by OLIGOURIDYLATE-BINDING PROTEIN 1 contributes to translational control during hypoxia in Arabidopsis. Proc Natl Acad Sci USA 111:2373-2378.

Souret, F. F., Kastenmayer, J. P., and Green, P. J. 2004. AtXRN4 degrades mRNA in Arabidopsis and its substrates include selected miRNA targets. Mol Cell 15:173-183.

Stanek, D. 2017. Cajal bodies and snRNPs - friends with benefits. RNA Biol 14:671-679.

Steffens, A., Jaegle, B., Tresch, A., Hulskamp, M., and Jakoby, M. 2014. Processing-body movement in Arabidopsis depends on an interaction between myosins and Decapping protein1. Plant Physiol 164:1879-1892.

Stępiński, D. 2014. Functional ultrastructure of the plant nucleolus. Protoplasma 251:1285-1306.

Stevaert, A., and Naesens, L. 2016. The Influenza virus polymerase complex: an update on its structure, functions, and significance for antiviral drug design. Med Res Rev 36:1127-1173.

Stoecklin, G., and Kedersha, N. 2013. Relationship of GW/P bodies with stress granules. Adv Exp Med Biol 768:197-211.

Sulkowska, A., Auber, A., Sikorski, P. J., Silhavy, D. N., Auth, M., Sitkiewicz, E., Jean, V., Merret, R. M., Bousquet-Antonelli, C. C., and Kufel, J. 2020. RNA helicases from the DEA(D/H)-Box family contribute to plant NMD efficiency. Plant Cell Physiol 61:144-157.

Swale, C., Da Costa, B., Sedano, L., Garzoni, F., McCarthy, A. A., Berger, I., Bieniossek, C., Ruigrok, R. W. H., Delmas, B., and Crépin, T. 2020. X-ray structure of the human karyopherin RanBP5, an essential factor for influenza polymerase nuclear trafficking. J Mol Biol 432:3353-3359.

\section{T}

Takata, M. A., Goncalves-Carneiro, D., Zang, T. M., Soll, S. J., York, A., Blanco-Melo, D., and Bieniasz, P. D. 2017. CG dinucleotide suppression enables antiviral defence targeting non-self RNA. Nature 550:124-127. 
Taliansky, M. E., Brown, J. W. S., Rajamäki, M. L., Valkonen, J. P. T., \& Kalinina, N. O. (2010). Involvement of the plant nucleolus in virus and viroid infections: parallels with animal pathosystems. Advances in virus research, 77, 119-158.

Taubenberger, J. K., and Morens, D. M. 2008. The pathology of influenza virus infections. Annu Rev Pathol 3:499-522.

Taylor, S. L., Frias-Staheli, N., García-Sastre, A., and Schmaljohn, C. S. 2009. Hantaan virus nucleocapsid protein binds to importin alpha proteins and inhibits tumor necrosis factor alpha-induced activation of nuclear factor kappa B. J Virol 83:1271-1279.

Teixeira, D. 2005. Processing bodies require RNA for assembly and contain nontranslating mRNAs. Rna 11:371-382.

Tharun, S., Muhlrad, D., Chowdhury, A., and Parker, R. 2005. Mutations in the Saccharomyces cerevisiae LSM1 gene that affect mRNA decapping and 3' end protection. Genetics 170:33-46.

Thran, M., Link, K., and Sonnewald, U. 2012. The Arabidopsis DCP2 gene is required for proper mRNA turnover and prevents transgene silencing in Arabidopsis. Plant J 72:368-377.

Topisirovic, I., Siddiqui, N., Lapointe, V. L., Trost, M., Thibault, P., Bangeranye, C., Piñol-Roma, S., and Borden, K. L. 2009. Molecular dissection of the eukaryotic initiation factor 4E (elF4E) exportcompetent RNP. EMBO J 28:1087-1098.

Torres, M. A., Jones, J. D., and Dangl, J. L. 2006. Reactive oxygen species signalling in response to pathogens. Plant Physiol 141:373-378.

Trinkle-Mulcahy, L., and Sleeman, J. E. 2016. The Cajal body and the nucleolus: "In a relationship" or "It's complicated"? RNA Biology 14:739-751.

Tripathi, D., Raikhy, G., Goodin, M. M., Dietzgen, R. G., and Pappu, H. R. 2015. In vivo localization of iris yellow spot tospovirus (Bunyaviridae)-encoded proteins and identification of interacting regions of nucleocapsid and movement proteins. PLoS One 10:e0118973.

Tsai, W. C., and Lloyd, R. E. 2014b. Cytoplasmic RNA Granules and Viral Infection. Annu Rev Virol 1:147-170.

Tsuzuki, M., Motomura, K., Kumakura, N., and Takeda, A. 2017. Interconnections between mRNA degradation and RDR-dependent siRNA production in mRNA turnover in plants. J Plant Res 130:211-226.

U

Uhrig, J. F., Canto, T., Marshall, D., and MacFarlane, S. A. 2004. Relocalization of nuclear ALY proteins to the cytoplasm by the Tomato bushy stunt virus P19 pathogenicity protein. Plant Physiol 135:2411-2423.

Unterholzner, L., and Izaurralde, E. 2004. SMG7 acts as a molecular link between mRNA surveillance and mRNA decay. Mol Cell 16:587-596.

\section{V}

van Dijk, E., Cougot, N., Meyer, S., Babajko, S., Wahle, E., and Seraphin, B. 2002. Human Dcp2: a catalytically active mRNA decapping enzyme located in specific cytoplasmic structures. EMBO J 21:6915-6924.

van Knippenberg, I., Goldbach, R., and Kormelink, R. 2002. Purified tomato spotted wilt virus particles support both genome replication and transcription in vitro. Virology 303:278-286.

van Knippenberg, I., Lamine, M., Goldbach, R., and Kormelink, R. 2005. Tomato spotted wilt virus transcriptase in vitro displays a preference for cap donors with multiple base complementarity to the viral template. Virology 335:122-130.

Vogel, D., Rosenthal, M., Gogrefe, N., Reindl, S., and Günther, S. 2019. Biochemical characterization of the Lassa virus L protein. J Biol Chem 294:8088-8100. 


\section{W}

Wang, A., and Krishnaswamy, S. 2012. Eukaryotic translation initiation factor 4E-mediated recessive resistance to plant viruses and its utility in crop improvement. Mol Plant Pathol 13:795-803.

Wang, L., Tan, H., Wu, M., Jimenez-Gongora, T., Tan, L., and Lozano-Duran, R. 2017a. Dynamic virusdependent subnuclear localization of the capsid protein from a geminivirus. Front Plant Sci 8:2165.

Wang, L. L., Wang, X. R., Wei, X. M., Huang, H., Wu, J. X., Chen, X. X., Liu, S. S., and Wang, X. W. 2016. The autophagy pathway participates in resistance to Tomato yellow leaf curl virus infection in whiteflies. Autophagy 12:1560-1574.

Wang, X., Chang, L., Wang, H., Su, A., and Wu, Z. 2017b. Dcp1a and GW182 induce distinct cellular aggregates and have different effects on microrna pathway. DNA Cell Biol 36:565-570.

Wang, Y., Zhou, J., and Du, Y. 2014. hnRNP A2/B1 interacts with influenza A viral protein NS1 and inhibits virus replication potentially through suppressing NS1 RNA/protein levels and NS1 mRNA nuclear export. Virology 449:53-61.

Weber, C., Nover, L., and Fauth, M. 2008. Plant stress granules and mRNA processing bodies are distinct from heat stress granules. Plant J 56:517-530.

White, J. P., and Lloyd, R. E. 2012. Regulation of stress granules in virus systems. Trends Microbiol 20:175-183.

Widana Gamage, S. M. K., and Dietzgen, R. G. 2017. Intracellular localization, interactions and functions of Capsicum chlorosis virus proteins. Front Microbiol 8:612.

Wilkie, G. S., Dickson, K. S., and Gray, N. K. 2003. Regulation of mRNA translation by 5'-and 3'-UTRbinding factors. Trends Biochem Sci 28:182-188.

\section{$\mathrm{X}$}

Xiang, Y., Kakani, K., Reade, R., Hui, E., and Rochon, D. 2006. A 38-amino-acid sequence encompassing the arm domain of the cucumber necrosis virus coat protein functions as a chloroplast transit peptide in infected plants. J Virol 80:7952-7964.

Xie, L., Lu, B., Zheng, Z., Miao, Y., Liu, Y., Zhang, Y., ... \& Wang, H. (2018). The 3C protease of enterovirus A71 counteracts the activity of host zinc-finger antiviral protein (ZAP). Journal of General Virology, 99(1), 73-85.

$\mathrm{Xu}$, J., and Chua, N.-H. 2009a. Arabidopsis decapping 5 is required for mRNA decapping, P body formation, and translational repression during postembryonic development. The Plant cell 21:3270-3279.

Xu, J., and Chua, N. H. 2011. Processing bodies and plant development. Curr Opin Plant Biol 14:88-93.

Xu, J., Yang, J. Y., Niu, Q. W., and Chua, N. H. 2006. Arabidopsis DCP2, DCP1, and VARICOSE form a decapping complex required for postembryonic development. Plant Cell 18:3386-3398.

\section{$Y$}

Yao, M., Zhang, T., Zhou, T., Zhou, Y., Zhou, X., and Tao, X. 2012. Repetitive prime-and-realign mechanism converts short capped RNA leaders into longer ones that may be more suitable for elongation during rice stripe virus transcription initiation. J Gen Virol 93:194-202.

Ye, J., Yang, J., Sun, Y., Zhao, P., Gao, S., Jung, C., Qu, J., Fang, R., and Chua, N. H. 2015. Geminivirus activates asymmetric leaves 2 to accelerate cytoplasmic DCP2-Mediated mRNA turnover and weakens RNA silencing in Arabidopsis. PLoS Pathog 11:e1005196.

Yu, G., Wang, L. G., Han, Y., and He, Q. Y. 2012. clusterProfiler: an R package for comparing biological themes among gene clusters. Omics : a journal of integrative biology 16:284-287. 
Yuan, P., Bartlam, M., Lou, Z., Chen, S., Zhou, J., He, X., Lv, Z., Ge, R., Li, X., Deng, T., Fodor, E., Rao, Z., and Liu, Y. 2009. Crystal structure of an avian influenza polymerase PA(N) reveals an endonuclease active site. Nature 458:909-913.

\section{Z}

Zhang, X., Yuan, Y. R., Pei, Y., Lin, S. S., Tuschl, T., Patel, D. J., and Chua, N. H. 2006. Cucumber mosaic virus-encoded $2 \mathrm{~b}$ suppressor inhibits Arabidopsis Argonaute1 cleavage activity to counter plant defense. Genes Dev 20:3255-3268.

Zhang, X. P., Liu, D. S., Yan, T., Fang, X. D., Dong, K., Xu, J., Wang, Y., Yu, J. L., and Wang, X. B. 2017. Cucumber mosaic virus coat protein modulates the accumulation of $2 \mathrm{~b}$ protein and antiviral silencing that causes symptom recovery in planta. PLoS Pathog 13:e1006522.

Zhao, J., Zhang, X., Hong, Y., and Liu, Y. 2016. Chloroplast in plant-virus interaction. Front Microbiol 7:1565.

Zhao, S., Xu, G., He, G., Peng, Y., and Liang, C. 2019. Characterization of an endonuclease in rice stripe tenuivirus Pc1 in vitro. Virus Res 260:33-37.

Zheng, L., Du, Z., Lin, C., Mao, Q., Wu, K., Wu, J., Wei, T., Wu, Z., and Xie, L. 2015. Rice stripe tenuivirus p2 may recruit or manipulate nucleolar functions through an interaction with fibrillarin to promote virus systemic movement. Mol Plant Pathol 16:921-930.

Zhou, M., and Law, J. A. 2015. RNA Pol IV and V in gene silencing: Rebel polymerases evolving away from Pol II's rules. Curr Opin Plant Biol 27:154-164. 


\section{Summary}

Viruses are obligate intracellular parasites, which have to utilize the host machinery to accomplish transcription, translation and replication, in order to produce virus progeny. For segmented, negative stranded RNA viruses (NSVs), cap-snatching is a unique process to facilitate viral genome transcription initiation. The studies on cap-snatching for both cytoplasmic- and nuclear-replicating viruses have revealed some common features, but there are also some differences and the most striking one relates to the source from where these viruses take capped-RNA leaders to prime mRNA synthesis. For the nuclear-replicating Influenza viruses, cap-snatching occurs at RNA polymerase II (pollI) sites, where the viral transcriptase complex, bound at the C-terminal domain of the host RNA polll, is able to directly access to $5^{\prime}$ capped leader sequences from nascent mRNAs. At the start of this thesis research, a few studies pointed to cytoplasmic $P$ bodies as a source from where the cytoplasmic NSVs would steal capped-RNA leaders. However, there were still questions that could not be answered and pointed to a situation that was likely more complex (Chapter 1). In order to better understand the various cytoplasmic RNA granules and indicate their potential as a source of cap donors for the cytoplasmic-replicating NSVs, an overview was made of the different cellular RNA granules and their functions in the regulation of cellular processes and control of cell development (Chapter 2). In addition, the interplay of these granular structures with viruses during an infection, as well as their pro- and antiviral activity was described.

To get a first glimpse of the source of host-derived capped-RNA leaders, an RNA-seq experiment was performed to analyse the host-derived capped-RNA leaders at the 5' end of viral mRNAs, and identify their corresponding host genes (Chapter $\mathbf{3}$ ). Whereas viral transcripts were identified within the transcriptome, capped RNA leader sequences derived from host cellular mRNAs were hardly collected. Likely, their absence resulted from the experimental approach to make a cDNA library, and failure to copy complete $5^{\prime}$ UTR sequences up to the $5^{\prime}$ cap. On the other hand, the transcriptome data revealed a change in the gene expression profile induced upon viral infection, in which genes corresponding to the photosynthesis pathway were downregulated the most (Chapter $\mathbf{3}$ ).

Earlier studies revealed a colocalization of the hantavirus Sin nombre virus (SNV) N protein with $\mathrm{P}$ bodies (PBs), and an affinity of this protein to $5^{\prime}$ caps. Therefore, it was postulated that this affinity likely led to a localization at foci enriched for capped RNA. If true, it was 
speculated that the SNV N protein might also co-localize with stress granules, which contain functional mRNAs stalled in their translation and are intimately related to PBs. To test this hypothesis for bunyaviruses and analyse for generic features, in situ localization studies were performed on bunyaviral $\mathrm{N}$ proteins from distinct animal- and plant infecting viruses. The results showed that the $\mathrm{N}$ proteins analysed all co-localized with PBs and SGs (Chapter 4). Furthermore, for Tomato spotted wilt virus (TSWV) and Schmallenberg virus (SBV) this was confirmed to occur during a natural infection as well. Interestingly, a preference of TSWV and SBV N protein was observed for the PB-SG docking complex. When the PB enzymatic factor DCP5 gene was silenced, slowing down the de-capping and degradation of mRNA, viral titres went up. However, when the NMD pathway factor UPF1 gene was silenced, assumed to reduce the influx of non-functional mRNAs into PB, viral titres went down only slightly. When the SG formation-related factors G3BP1-like and Rbp47 were silenced, viral titres increased. When the translation initiation factors elF4E and elF4A (SG resident components) were silenced, viral titres were positively or negatively affected, respectively. Upon simultaneous silencing of both DCP5 and G3BP1-like genes, TSWV was able to replicate better than in control plants, but less to a lesser extent than when the additive effect of the individually silenced genes. Altogether, these results indicated that the role of PBs as the only/major source from where these viruses could collect capped RNA could be questioned. To test for the use of possible upstream sources of cytosolic capped RNAs, the localization of the TSWV N protein was analysed relative to the perinuclear region. A co-localization of $\mathrm{N}$ with RanGAP2, a nuclear envelope and nucleocytoplasmic shuttling factor, was observed, while the $\mathrm{N}$ protein also seemed to interact with a particular domain of RanGAP2. When both RanGAP homologs in plants were silenced, TSWV titres went clearly down, pointing to the nuclear pore complex as a potential site from where these viruses could access cappedRNA leaders from the nuclear mRNA efflux (Chapter 4).

As a complementary approach and alternative to find clues that would point to the source of host-derived capped-RNA leaders, high throughput sequencing (HTS) was applied on a large number of capped-leader sequences from viral mRNAs (Chapter 5). By bioinformatic analysis, capped-leader sequences were analysed and their donor transcripts identified. Host leader sequences snatched were generally between 16-20 nucleotides long. Most of the leader sequences ended up with AGA or GAG and it appeared that certain motifs were used more frequently. Analysis of the identified host donor transcripts revealed the abundant use of photosynthesis gene transcripts for TSWV cap-snatching. It was also found that heat stress (SG-induction) altered the usage of certain gene transcripts (Chapter 5). 
When functional GFP and non-functional GFP transcripts were simultaneously offered as cap donors in TSWV-infected plants both transcripts were used during TSWV cap-snatching. When a functional GFP transcript and a long GFP transcript were offered during a TSWV infection in planta, at normal and stress conditions, again both leaders were being used during cap-snatching. The results altogether indicated that TSWV was able to use transcripts destined to PBs and SGs, but also from possible upstream sources containing functional GFP transcripts (Chapter 5).

In summary, the research described in this thesis indicates that the cytoplasmic-replicating segmented NSVs likely apply a generic mode of cap-snatching with a potential role for $\mathrm{P}$ bodies and stress granules, but also the nucleopore complexes, as source and foci from where capped-donor RNAs may be used, as being discussed in Chapter 6. The abundant usage of transcripts from photosynthesis pathway genes during TSWV cap-snatching indicates that, although somewhat speculative, disease symptoms like chlorosis could at least in part, be the result of a specific translational shut-off by plant-infecting NSVs, resulting from cap-snatching from host mRNAs needed for proteins effective in photosynthesis. Further evidence, whether capped-mRNAs indeed are being used from the aforementioned granules/foci will have to come from future co-localization studies with the viral RdRp protein, the viral protein needed for endonuclease cleavage of leader sequences. 


\section{Acknowledgements}

Here, I finally come to the end of my PhD life, it was a unique journey with a lot of joy, struggles, endeavours and support. I feel lucky to have had this experience at both Nanjing Agricultural University and Wageningen University, and to contribute to the collaboration of two plant virus research groups. I would like to take this opportunity to thank all the people that have accompanied, helped, inspired, and loved me in this journey.

陶老师, thank you for providing me this opportunity to go abroad for such an amazing experience. You are the enlightener of my research career. You showed great passion to the research from the first time I met you, and such passion stays and never fades. From a young and naïve girl, you taught me about TSWV and frontiers in science, and I learned to explore this world in a proactive and scientific manner. Although this process is rather slow, you never gave up and always kept supporting me. Thank you for helping me and respecting my decisions all the time.

Richard, I still remember the first time I met you in Nanjing (you did not remember me, unfortunately:)). I could not imagine to be your student by that time, but how lucky I was when this came true. If it was not you as my supervisor, I would not have decided to stay in Wageningen longer. Thank you for training me how to perform research and how to tell a scientific story to an audience. I learned a lot from you. You are also a good friend, I enjoyed every talk with you and appreciate your inspirations and supports (also your cooking).

Monique, thank you for having me back to virology to finish my PhD. I enjoyed all the time together with you and wish I could have had more contact with you. I appreciate your visit to Nanjing for my PhD continuation and I miss the time we spent together visiting the attractions and sharing our minds on everything. You are a super nice group leader, arranging everything considerately and making the laboratory of virology a pleasant environment to work. Thank you for helping me with the revision of my thesis and all the comments to my research.

Judith, thank you for helping me with the bioinformatic analysis. You are a brilliant bioinformatician. Without your help, I would not have easily completed my thesis in time. It was a pity that due to the covid situation, we were not able to meet physically. I hope that someday in the near future we will be able to meet in person and communicate face-to-face and who knows, collaborate in future research. 
My lovely plant buddies, I enjoyed all the time being with all of you and appreciated all the help you provided. Corien, I was so happy when I saw the moment you finished your PhD and entered the next phase of your scientific career with a job as plant virologist at one of the Dutch plant research stations. Thank you for your help during my start-up period with all the introductions and sharing of material. Andre, thank you for all the help in the lab. I had great moments with you and Karol. The "IELTS" book you gave to me helped me a lot with my English test. You both are always nice to me and I feel very relaxed when (coming over to your place and) being with you. Irene, I am so glad that you were around when I came back to Wageningen. I enjoyed our time together in the office and lab. I hope you also will enjoy your last months of your PhD life like I have done, and wish you good luck and success when finishing it. Magda, I am happy that we worked on the same project. Although we worked on it by each of it using different cell systems, it made the project challenging. You are also a very nice friend and I liked our talks. Dick, thank you for the warmness when I started on the lab in Wageningen and for all your help in the lab. Cristina, you are the one that always helps me without hesitation and I always appreciated that, besides our chats of course. Maria, although we have only spent some brief time in the lab, your smile has always been inspiring and I hope you are enjoying your work and life back in Spain, with family and friends again. Sharella, it was a big and nice surprise to see you doing a PhD in virology group when I came back, after I witnessed you earlier as a student in one of the virology courses. I hope ("but I am sure") you will enjoy it. Emilyn, I am happy that you have joined the plant virology family. Even though I am finishing my time at the Laboratory of Virology, I do get the chance to already learn you more personal. Dennis, thanks for your efforts to organise a small lab-trip to socialize and help Cristina to manage the plant virus lab. Rene and Karen, I enjoyed the discussions and chats with you during our plant group meetings. Mandy, I put you at last but not because you are the least. Thanks for being my paranymph and helping me to organize this event. I feel honoured to have you as a colleague and as a friend. You are kind, considerate and smart, and always bring me a lot of energy. I hope that one of these days we will find an opportunity to travel to Korea or China together.

Corrine and Els, thank you for helping me with the use of lab facilities. Marleen, thank you for helping me with the ordering of biochemicals and so on. Without all of your help, I could not have done my experiments smoothly. Gwen and Dorothy, it was nice to see that you joined the team of technicians and in your own way contribute to a very pleasant and great environment in the lab. Melanie, thank you for helping me with all the administrative stuff without any complaints. 
Of course I would like to thank everyone and all the people that make virology a great place to work, the virology events and all the conversations we had will always be cherished and never be forgotten. Vera, Jan, Gorben, Jeroen, and Jelke, thanks for the many nice discussions during Monday morning seminars and chats during coffee time/lunch break. Han, Fengqiao, Aydin, Gabriela, Simone, Sandra, Miao, Giel, Bob, Jitte, Tessy, Haidong, Irene Meki, Haozhou, John, Jerome, Linda, Joyce, Astrid, Xiao, Ahmad, Annamaria, Jirka, Hanna, Janna, Luzhao, Carolina, thanks for all the fun and things we did together and of course all the support I received from you.

It was also nice to have our PRI neighbors around. Jan B, thanks for helping me to contact on the use of MinION. Annette, thank you for helping me with lab access. I would also like to thank Marga for the help with the use of the qPCR machine. Thanks Norbert and 郑颖老师 for the introduction and technical support on confocal microscopy. My special thanks go to Henk, you've always been a magician to me because no matter when, you were always able to provide me with sufficient plants for my experiments, even last minute. Without your kind help, I wouldn't have finished my experiments in time.

Also thanks to my students Marco and Raquel. Thank you for your contribution to the project, you were the best students I could wish. Jingyi, Prianka, Mariangela, Ahmad, Yoga, Christina, although you were not my students, you are my friends. We had a great time together.

Aska, thanks for being my external advisor. I feel lucky that I did not run into problems with my supervisors so I didn't have to trouble you. Thank you and Hein for providing the materials for my project. Douwe and Susan, thanks for helping me with the EPS administration. Juliana, thanks for all your efforts to organize amazing EPS events.

中国小伙伴们, 跟你们在一起的时光都是无比快乐和难忘的。韩哥, 旭哥, 凡哥, 林啸师兄, 枫桥师兄, 田卉师姐, 锦斌师兄, 感谢大家在我初到荷兰时对我的照 顾。新芳, 王星, 感谢你们最初的陪伴, 我们一起度过了很多美好的时光。华一, 梦婧, 雪雪, 小雪, 杜老师, 黄老师, 郭老师, 吴怡, 张冬, 子琳, 孟鹤姐, 静 静, 成成, 崔否师兄, 闵哲师姐以及整个育种组大家庭, 感谢你们的接纳, 认识大 家是我的荣幸。 
琪姐, 感谢做我的paranymph, 这四年来参加了无数次你组的局, 收获了无数回忆和 欢笑, 很开心一路有你的温暖陪伴, 希望接也能陪伴你走完接下来的PhD生涯。

缪, 这次一起回来, 跟你相处的时间最长, 在组里, 我们互相陪伴做实验, 日常 中, 我们一起逛街, 做饭, 出游, 很荣幸能在博士最后一年里交到一个知心朋友, 谢谢你的包容理解鼓励支持。

雨晴妹妹, 伟钊, 大哥, 碧瑶, 若璇, 黄雯, 廉洁, 王琪, 李鲁兆, 彦君, 珊珊, 张陈, 曾哲, 跟你们聊天吃饭, 喝酒打牌, 一起的时光短暂却美好。

南京的家人们, 你们的期盼和等待是我前进的动力。朱老师, 佳哥, 徐老师谢谢你 们在科研中的指引和在生活中的关怀。冯师兄, 蒋否师兄, 小娇师姐, 吴鉴艳师 姐, 黄莹师姐, 赵小慧师姐, 薛凡师姐, 黄莹师姐, 钱新, 金渏倩, 海宁, 洪浩, 小强, 冯明峰, 陈虹宇, 佟聪, 静哥, 吴师姐, 慧姐, 项青, 春莉, 吴茜, 赵延 晓, 杨同庆, 黄申, 问娇玲, 郭荣, 路遥, 陈明龙, 小蔡, 张显、左崇坤、喻露 莎、戴婧、董永金金、程锐祥、苗丹和蔡利娜以及所有实验室的小伙伴们, 感谢大家 的陪伴与支持。

密密, 玉梅, 庆, 你们虽然早我一步踏入社会, 但你们的友情一直伴随着我, 谢谢 你们时不时地给我加油打气, 你们是我坚强的后盾。

爸爸妈妈, 谢谢你们尊重我的每一个决定, 支持我的每一个选择, 给我一个温暖的 避风港, 让我能无忧的追逐自己的梦想。

冬, 很幸运能够遇见你, 你的鼓励和陪伴让异国他乡的博士生涯变得不再那么难 謷, 你的耐心体贴让我脾气不再那么暴躁, 你的认真上进不断引领着我, 你的温暖 善良时刻感染着我, 谢谢你, 让我的生活全都是阳光和美好, 希望我们能一起克服 这世间的重重困难，体验丰富多彩的人生。 


\section{About the author}

Min Xu (徐敏) was born on the 16th of November 1991 in the city of Laixi, Shangdong Province, China. In 2010, She started her bachelor study in Plant Protection at Qingdao Agricultural University. There, she completed her bachelor thesis titled" the isolation and identification of endophytes in Artemisia argyi". During her bachelor study, she became interested in plant-pathogen interactions, especially in relation to plant viruses. After her BSc graduation in 2014, she continued her master study in the Molecular Virology group at Nanjing Agricultural University under the supervision of Prof. Dr Xiaorong Tao. Two-years later, she moved

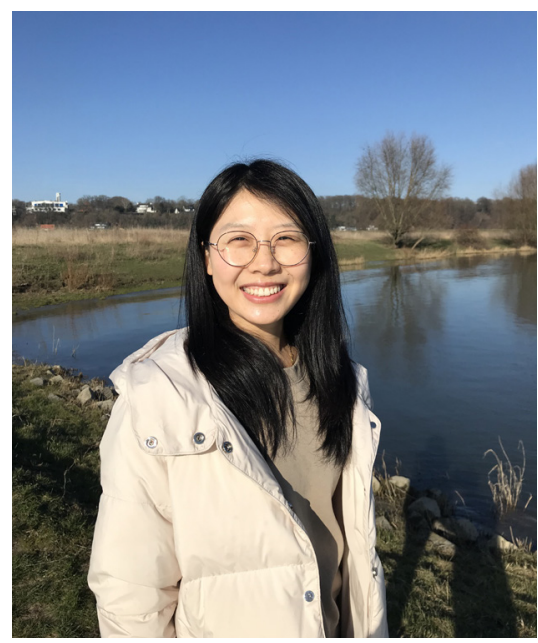
on to her doctoral study on the pathogenesis of Tomato spotted wilt virus (TSWV) in the same lab. One year later, she first visited Wageningen University, where she started to work in the Virology group, under the supervision of Dr Richard Kormelink and Prof. Dr Monique van Oers. From then onwards she focused on the cap-snatching mechanism of bunyaviruses besides TSWV. 


\section{Publication list}

Xu, M., Mazur, M. J., Nigel, G., Hong, H., Tao, X. \& Kormelink, R. (2021) Cytoplasmic sources of capped RNA for genome transcription initiation of cytoplasmic replicating, segmented negative strand RNA viruses. (In submission)

Xu, M., Judith R. \& Kormelink, R. (2021) High throughput sequencing of snatched host cellular mRNA leader sequences for viral genome transcription initiation and identification of the host donor genes. (In preparation)

Hong, H., Wang, C., Huang, Y., Xu, M., Feng, M., Li, J., Shi, Y., Zhu, M., Shen, D., Wu, P., Richard, K. \& Tao, X. (2021) Antiviral RISC mainly targets viral mRNA but not genomic RNA of tospovirus. PLoS Pathog (Accepted for publication)

Xu, M., Chen, J., Huang , Y., Sun, P., Shen, D. \& Tao, X. (2019) Dynamic transcriptional profiles of Arabidopsis thaliana infected by Tomato spotted wilt virus. Phytopathology 110:153-163.

Xu, M., Mazur, M.J., Tao, X. \& Kormelink R (2019) Cellular RNA hubs: friends and foes of plant viruses. Mol Plant Microbe Interact 33: 40-54.

Huang, Y., Hong, H., Xu, M. Y, J., Dai, J., Wu, J., Feng, Z., Zhu, M., Zhang, Z., Yuan, X., Ding, X. \& Tao, X. (2019) Developmentally regulated susceptibility of Arabidopsis thaliana to Tomato spotted wilt virus. Mol Plant Pathol 21:985-998.

Feng, Z., Xue, F., Xu, M., Chen, X., Hao, W., Garcia-Murria, M. J., Mingarro, I., Liu, Y., Huang, Y., Jiang, L., Zhu, M. \& Tao, X. (2016) The ER-Membrane Transport System Is Critical for Intercellular Trafficking of the NSm Movement Protein and Tomato Spotted Wilt Tospovirus. PLoS Pathog 12(2):e1005443 


\section{Education Statement of the Graduate School Experimental Plant Sciences}

Issued to:

Date:

Group:

University:
Min Xu

06 October 2021

Virology

Wageningen University \& Research

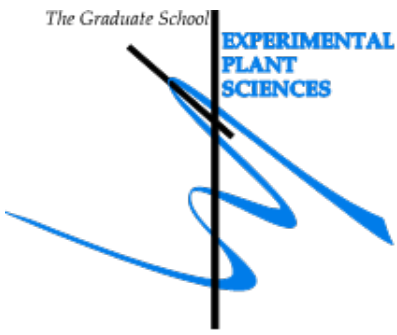

1) Start-Up Phase

date

cp

- First presentation of your project

A study into the roles of RNA granules for replication of segmented, negative $\quad 10$ Nov 2017 strand plant RNA viruses in planta

- Writing or rewriting a project proposal

Analysis of cytoplasmic RNA granules in the replication-transcription of segmented, negative strand RNA plant viruses

- MSc courses

Subtotal Start-Up Phase

2) Scientific Exposure 4.5

- EPS PhD student days

EPS PhD student days 'Get2Gether', Soest (NL)

11-12 Feb $2019 \quad 0.6$

EPS PhD student days 'Get2Gether', online

1-2 Feb 2021

0.4

- EPS theme symposia

EPS Theme 2 Symposium "Interactions between plants and biotic agents", 24 Jan 2018 Amsterdam (NL)

EPS Theme 3 Symposium "Metabolism and Adaptation", online

30 Oct 2020

EPS Theme 2 Symposium "Interactions between plants and biotic agents", online

9 Feb 2021

- Lunteren Days and other national platforms

Annual Meeting 'Experimental Plant Sciences', Lunteren (NL)

9-10 Apr $2018 \quad 0.6$

Annual Meeting 'Experimental Plant Sciences', online

12-13 Apr $2021 \quad 0.5$

Dutch Annual Virology Symposium (DAVS), Amsterdam (NL)

9 Mar 2018

Dutch Annual Virology Symposium (DAVS), online

5 Mar 2021

- Seminars (series), workshops and symposia

Insight of host factor Hsp70 in viral inhibition-roles in TBSV infection in

17 Jul 2017 Nicotiana Benthamiana and the role of sterols in tombusvirus replication Phospholipids positive-strand RNA virus replication Brome mosaic virus (BMV)

15 Aug 2017

Roles of Rab5 small GTPase in the biogenesis for tomato spotted bushy stunt

15 Aug 2017 virus replication organelles 


\section{Appendix}

Innovation scientific solutios to urban agricultural, sustainability and food 14 Mar 2019 production BAG7 regulate virus infection(TuMV, PIAMV)

MYC2/MED25 at the Nexus of Jasmonate signaling

17 May 2019

Broad spectrum resistance gene discovery? Mechanism and application

24 May 2019

Application of plant-fungal RNAi shift in cotton wilt disease

24 May 2019

Genome editing with programmable nucleus in crop plants

5 Jul 2019

Evolution of DNA methylation and RNA-seq differential analisis methods

10 Jul 2019

What's in a name? (on bunyavirus)

26 Sep 2019

0.1

Dissecting the Molecular Interplay between Tospoviruses and Thrips Vectors

26 Sep 2019

Research on Plant Rhabdovirus-Insect vector-Host Plant Interaction

28 Sep 2019

0.1

Organelle biogenesis and function in plant

29 Oct 2019

0.1

Plant-pathogen interactions facing a changing climate

17 Mar 2021

Defining tissue-specificity of plant colonization by bacteria

17 Mar 2021

Intimate Association of PRR- and NLR-Mediated Signaling in Plant Immunity

22 Mar 2021

0.1

Dual Role of Auxin in Regulating Plant Defense and Bacterial Virulence Gene Expression During Pseudomonas syringae PtoDC3000 Pathogenesis

12 Apr 2021

How do plants engage with beneficial microorganisms while at the same time

3 May 2021 restricting pathogens

Symposium on plant-resistance and viral pathogenicity, Nanjing (CN)

30-31 Dec 2016

Symposium on plant-resistance and viral pathogenicity, Nanjing (CN)

30-31 Dec 2019

- Seminar plus

Discussion on Plant-TSWV interaction with dr. Frank Takken and dr. Harrold van den Burg

16 Aug 2017

Discussion on Molecular Interplay between Tospoviruses and Thrips with dr. 26 Sep 2019 Anna Whitfield

- International symposia and congresses

International Symposium on Plant Immunity, Nanjing (CN)

24-26 Jun 2019

The $\mathrm{XI}^{\text {th }}$ International Symposium on Thysanoptera and Tospoviruses, Kunming (CN)

- Presentations

The XIth International Symposium on Thysanoptera and Tospoviruses, Kunming

21-25 Sep 2019 $(\mathrm{CN})$, oral presentation

EPS PhD student days 'Get2Gether', online, oral presentation

1 Feb 2021

- 3rd year interview

- Excursions

ZEISS Innovation Center for Research and Development, Shanghai (CN)

14 Jan 2020

0.3

Rijk Zwaan, online

16 Jun 2021

0.1

Subtotal Scientific Exposure

3) In-Depth Studies

11.5

- Advanced scientific courses \& workshops

Plant Molecular Immunology, Nanjing (CN)

Mar-Aug, $2017 \quad 2.0$ 
Molecular Biology of Plant Pathogen, Nanjing (CN)

Mar-Aug, $2017 \quad 2.0$

The Power of RNA-seq, Wageningen (NL)

11-13 Jun $2018 \quad 0.9$

Electron Microscopy Course, Amsterdam \& Wageningen (NL)

12-16 Nov $2018 \quad 1.6$

- Journal club

PhD discussion group for project progress and literature sharing (weekly)

Mar-Dec 2019

0.7

- Individual research training

Bioinformatics training for RNA-seq data analysis, Phytophthora group, Nanjing Jun 2017

Agricultural University, Nanjing (CN)

Subtotal In-Depth Studies

4) Personal Development

date

cp

- General skill training courses

Course - Searching and Organising Literature, Wageningen (NL)

23-24 Oct, $2018 \quad 0.6$

Course - Introduction to R for Statistical Analysis, Wageningen (NL)

8-9 Nov $2018 \quad 0.6$

Course - Scientific Writing, Wageningen (NL)

9 Mar-7 May 20201.8

Workshop - How to Engage in Meaningful Conversations? Introducing the WUR

17 May 2021

Dialogue Navigator, online

Workshop - Managing your supervisor in an online world, online

18 May 2021

Workshop - Academics outside academia?! Food \& Agri edition, online

21 May 2021

Workshop - Drawing essentials for impactful communication, peace of mind

25 May 2021 and lots of fun, online

- Organisation of meetings, PhD courses or outreach activities

Organisation of the XIth International Symposium on Thysanoptera and Sep 2019 Tospoviruses, Kunming (CN)

- Membership of EPS PhD Council

Subtotal Personal Development

5) Teaching \& Supervision Duties

date

cp

- Courses

- Supervision of BSc/MSc students

Supervision of MSc student, Marco Jansen

2018/2019

Supervision of MSc student, Raquel Kooijman

Subtotal Teaching \& Supervision Duties

TOTAL NUMBER OF CREDIT POINTS*

Herewith the Graduate School declares that the PhD candidate has complied with the educational requirements set by the Educational Committee of EPS with a minimum total of 30 ECTS credits.

* A credit represents a normative study load of 28 hours of study. 
This research was performed at both Virology, Wageningen University \& Research and Molecular Plant Virology, Nanjing Agricultural University. Min Xu was sponsored by a PhD fellowship from the China Scholarship Council (CSC, No. 201706850041).

Cover design and thesis layout by Yidong Wang

Printed by GVO drukkers \& vormgevers B.V., Ede, the Netherlands 